\title{
Multi-scale simulations of particle acceleration in astrophysical systems
}

\section{Alexandre Marcowith ${ }^{1} \cdot$ Gilles Ferrand $^{2} \cdot$ Mickael Grech $^{3} \cdot$ Zakaria Meliani $^{4}$. Illya Plotnikov 5 . Rolf Walder 6}

Received: 30 July 2019 / Accepted: 25 January 2020 / Published online: 23 March 2020

(c) The Author(s) 2020

\begin{abstract}
This review aims at providing an up-to-date status and a general introduction to the subject of the numerical study of energetic particle acceleration and transport in turbulent astrophysical flows. The subject is also complemented by a short overview of recent progresses obtained in the domain of laser plasma experiments. We review the main physical processes at the heart of the production of a non-thermal distribution in both Newtonian and relativistic astrophysical flows, namely the first and second order Fermi acceleration processes. We also discuss shock drift and surfing acceleration, two processes important in the context of particle injection in shock acceleration. We analyze with some details the particle-in-cell (PIC) approach used to describe particle kinetics. We review the main results obtained with PIC simulations in the recent years concerning particle acceleration at shocks and in reconnection events. The review discusses the solution of Fokker-Planck problems with application to the study of particle acceleration at shocks but also in hot coronal plasmas surrounding compact objects. We continue by considering large scale physics. We describe recent developments in magnetohydrodynamic (MHD) simulations. We give a special emphasis on the way energetic particle dynamics can be coupled to MHD solutions either using a multi-fluid calculation or directly coupling kinetic and fluid calculations. This aspect is mandatory to investigate the acceleration of particles in the deep relativistic regimes to explain the highest cosmic ray energies.
\end{abstract}

Keywords Kinetic physics · Magnetohydrodynamics $\cdot$ Turbulence $\cdot$ Particle acceleration

\section{Contents}

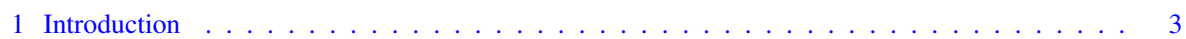

1.1 Layout . . . . . . . . . . . . . . . . . . . . . . . . 6

1.2 List of acronyms and notations . . . . . . . . . . . . . . . . . . . . . 7

2 Astrophysical and physical contexts . . . . . . . . . . . . . . . . . . . . 9

Extended author information available on the last page of the article 
2.1 Stochastic acceleration . . . . . . . . . . . . . . . . . . . . 10

2.2 Diffusive shock acceleration . . . . . . . . . . . . . . . . . . . 10

2.2.1 Fermi processes and building power-laws . . . . . . . . . . . . . . . 11

2.2.2 The transport equation and the diffusion coefficient . . . . . . . . . . . 12

2.2.3 The injection problem . . . . . . . . . . . . . . . . . . . . . . . . 14

2.2 .4 Back-reaction and non-linear effects . . . . . . . . . . . . . . . . . . 15

2.3 Shock drift and shock surfing acceleration . . . . . . . . . . . . . . . . . . . . . 17

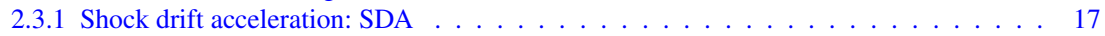

2.3.2 Shock surfing acceleration: SSA . . . . . . . . . . . . . . . . . . 18

2.4 Fermi acceleration process at relativistic shocks . . . . . . . . . . . . . . . . . . . . . 19

2.4.1 General statements . . . . . . . . . . . . . . . . . . . . . . . 19

2.4.2 Progress with fully kinetic simulations . . . . . . . . . . . . . . . . . . . . . . . . . . . . . . . . 22

2.4 .3 Long term evolution . . . . . . . . . . . . . . . . . . . . . . . . . . . . . . . . . . 22

2.5 Reconnection in astrophysical flows . . . . . . . . . . . . . . . . . . . . . . 22

2.5.1 Collisional reconnection models . . . . . . . . . . . . . . . . . . . 23

2.5 .2 Collisionless reconnection . . . . . . . . . . . . . . . . . . . . . . 28

2.5 .3 Other effects . . . . . . . . . . . . . . . . . . . . . . . 29

2.6 Laser plasma experiments . . . . . . . . . . . . . . . . . . . . . 30

2.6.1 Overview of laser facilities and characteristic parameters . . . . . . . . . . . 31

2.6 .2 The collisionless regime . . . . . . . . . . . . . . . . . . . . 33

2.6.3 Collisionless shock experiments . . . . . . . . . . . . . . . . . . . 34

2.6.4 Magnetic reconnection experiments . . . . . . . . . . . . . . . . . . . 41

3 Solving kinetic problems . . . . . . . . . . . . . . . . . . . . . . . . . . 44

3.1 The Vlasov-Maxwell description of a collisionless plasma . . . . . . . . . . . . . . . . 44

3.1 .1 Governing equations . . . . . . . . . . . . . . . . . . . . . . 44

3.1 .2 Initial and boundary conditions . . . . . . . . . . . . . . . . . . 46

3.2 Solving the Vlasov-Maxwell system numerically: general considerations . . . . . . . . . . . 46

3.2 .1 Initialization and time-loop . . . . . . . . . . . . . . . . . . . 46

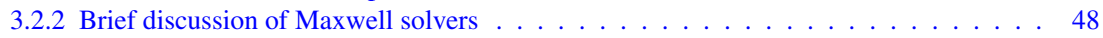

3.3 Particle-In-Cell codes . . . . . . . . . . . . . . . . . . . . . . . . . . . . . . . 49

3.3 .1 Method . . . . . . . . . . . . . . . . . . . . . 49

3.3 .2 Additional physics modules . . . . . . . . . . . . . . . . . . 50

3.3 .3 Stability issues: relativistic flows . . . . . . . . . . . . . . . . . . 51

3.3 .4 Examples . . . . . . . . . . . . . . . . . . . . . . 51

3.4 Vlasov-Maxwell codes . . . . . . . . . . . . . . . . . . . . . . 52

3.4 .1 PIC codes versus Vlasov codes . . . . . . . . . . . . . . . . . . . . 52

3.4 .2 The problem of filamentation in phase-space . . . . . . . . . . . . . . . 52

3.4.3 Example of different methods for electromagnetic Vlasov codes . . . . . . . . . . . 53

3.5 Hybrid methods . . . . . . . . . . . . . . . . . . . . . . . . . . 53

3.6 Solving Fokker-Planck problems . . . . . . . . . . . . . . . . . . . . . . . 54

3.6 .1 The Fokker-Planck equation . . . . . . . . . . . . . . . . . . . . 55

3.6 .2 Stochastic differential equations . . . . . . . . . . . . . . . . . . . 58

3.6.3 Simulating shock acceleration using a Monte-Carlo method . . . . . . . . . . . . . 61

4 Small and meso-scale numerical particle acceleration studies . . . . . . . . . . . . . . . . . . . 62

4.1 Shock acceleration numerical experiments: PIC simulations . . . . . . . . . . . . . . . . 62

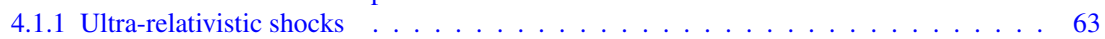

4.1 .2 Mildly relativistic shocks . . . . . . . . . . . . . . . . . . . . 71

4.1 .3 Non-relativistic shocks . . . . . . . . . . . . . . . . . . . . . 73

4.1 .4 Discussion . . . . . . . . . . . . . . . . . . . . . . . . . . . . . . . . . . . . 77

4.2 Kinetics of magnetic reconnection . . . . . . . . . . . . . . . . . . . . . 79

4.2.1 What kinetic simulations can achieve and why we decisively need them . . . . . . . 79

4.2.2 Some key results based on kinetic simulations . . . . . . . . . . . . . . . . . . . 84

4.2.3 Selected physical processes which accelerate particles . . . . . . . . . . . . . . 91 91

4.2 .4 A critical discussion and outlook . . . . . . . . . . . . . . . . . . . . . . . . . . . . . . . . . . . . . . . . . . . .

5 Macro scale numerical particle acceleration studies . . . . . . . . . . . . . . . . . . . 102

5.1 The equations of magnetohydrodynamics . . . . . . . . . . . . . . . . . 102

5.1 .1 Classical magnetohydrodynamics . . . . . . . . . . . . . . . . . 102 


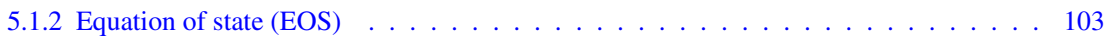

5.2 Numerical solutions . . . . . . . . . . . . . . . . . . . . . . . . . . . . . 103

5.2 .1 The Courant-Friedrichs-Lewy condition . . . . . . . . . . . . . 105

5.2 .2 Riemann solvers . . . . . . . . . . . . . . . . . . . . . . . . . . 105

5.2 .3 Semi-implicit and implicit schemes . . . . . . . . . . . . . . . . . . . . . 108

5.2 .4 Magnetic divergence-free algorithms . . . . . . . . . . . . . . . . . . . . . . . . . . 109

5.2 .5 Adaptive mesh refinement techniques . . . . . . . . . . . . . . . 110

5.2 .6 Errors estimator . . . . . . . . . . . . . . . . . . . . . . . . . . 111

5.2 .7 Load balance . . . . . . . . . . . . . . . . . . . . . . . . . . 111

5.2 .8 Standard numerical tests . . . . . . . . . . . . . . . . . . . . 112

5.2 .9 Relativistic magnetohydrodynamics . . . . . . . . . . . . . . . . . 113

5.3 Multi-fluid methods . . . . . . . . . . . . . . . . . . . . . . . . . . 115

5.3 .1 Model equations . . . . . . . . . . . . . . . . . . . . . . 116

5.3.2 Specific numerical schemes of CR-fluid systems . . . . . . . . . . . . . . . 117

5.3 .3 Numerical tests . . . . . . . . . . . . . . . . . . . . . . . . . . . . . . 120

5.3 .4 Cosmic ray physics using a bi-fluid approach . . . . . . . . . . . . . . 122

5.4 The fluid-kinetic framework . . . . . . . . . . . . . . . . . . . . . . . . . . . . 124

5.4 .1 Coupling hydro and diffusion-convection equations . . . . . . . . . . . . 125

5.4.2 Coupling MHD and Vlasov-Fokker-Planck equations _ . . . . . . . . . . . . 128

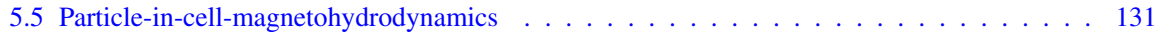

5.5 .1 Numerical schemes . . . . . . . . . . . . . . . . . . . . . . . . . . . . 133

5.5 .2 Coupling MHD and PIC time steps . . . . . . . . . . . . . . . . . . . . 134

5.5 .3 Adaptive mesh refinement for the PIC module . . . . . . . . . . . . . . . . . 134

5.5 .4 Numerical tests . . . . . . . . . . . . . . . . . . . . . . . . . . . 134

5.5.5 Results on cosmic ray acceleration at non-relativistic shocks . . . . . . . . . . 136

5.5 .6 Other astrophysical applications . . . . . . . . . . . . . . . . . . . . 139

5.6 A list of $\mathrm{HD}$ and MHD codes with CR physics . . . . . . . . . . . . . . . . . 140

5.7 Semi-analytical approaches for cosmic ray acceleration . . . . . . . . . . . . . . . . . 140

5.7 .1 General considerations . . . . . . . . . . . . . . . . . . . . . . 141

5.7 .2 Construction of a NLDSA model . . . . . . . . . . . . . . . . . . . . . . 143

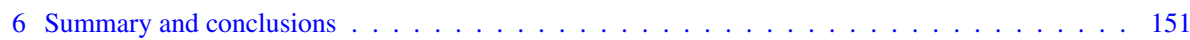

References . . . . . . . . . . . . . . . . . . . . . . . 153

\section{Introduction}

Particle acceleration is a widespread process in astrophysical, space and laser plasmas. Acceleration results from the effect of electric fields, but supra-thermal particles gain energy because their residence time in the acceleration zone increases due to magnetic confinement. Hence, particle acceleration is an electromagnetic process. Particle acceleration can be classified into three main sub-types (Blandford 1994; Kirk 1994; Melrose 1996): acceleration at flow discontinuities among which shock waves, stochastic acceleration, acceleration by direct electric fields. This last mechanism occurs in the environment of fast rotating magnetized objects like pulsars or planetary magnetosphere. It will not be discussed in this review, interested readers can refer to Cerutti and Beloborodov (2017) and references therein for what concerns pulsar magnetospheric physics. Some recent discussions concerning particle acceleration in Jupiter, the fastest rotator among solar system planets and other giant planet magnetospheres can be found in Mauk et al. (2017), Delamere et al. (2015).

As stated above acceleration processes can be classified into different categories. Let us give here a short overview of the main mechanisms. 
1. Stochastic Fermi acceleration (SFA). This is historically the first discovered acceleration process. SFA is at the heart of Fermi's work on the origin of cosmic rays (CRs) (Fermi 1949, 1954). In its original description particles with speed $v$ gain energy through a stochastic interaction with the convective electric field carried by magnetic clouds moving randomly at a speed $U \ll v$ in the interstellar space (see Sect. 2.2.1). This process has some well-known caveats: it is inefficient as the mean relative energy gain $\langle\Delta E / E\rangle \propto(U / v)^{2}$ (Parker 1958a), it produces non-universal power-law solutions (see Eq. 3), which can not explain the power-law distribution of CRs observed at Earth. A modern description of SFA includes randomly moving electromagnetic waves (Hall and Sturrock 1967), usually in the magnetohydrodynamic (MHD) limit if we want to consider the issue of CR acceleration (Parker 1955; Kulsrud and Pearce 1969). For SFA by MHD waves, the relevant speed for the scattering centers usually are proportional to the local Alfvén speed $U_{\mathrm{A}} \cdot{ }^{1} \mathrm{SFA}$ is more efficient if the speed of the scattering center is close to the speed of light (Marcowith et al. 1997; Gialis and Pelletier 2004) or for particles with speeds close to $U_{\mathrm{A}}$, or other plasma characteristic wave phase speeds as it is likely the case for low-energy CRs which propagate in the interstellar medium (ISM) (e.g., Ptuskin et al. 2006), in the solar corona (e.g., Miller et al. 1996; Pryadko and Petrosian 1998) or in the heliosphere (Zhang and Lee 2013). SFA is a multi-scale process if the scattering waves are distributed over large wave-number bands as it is the case in turbulent flows. SFA is an important process at the origin of particle acceleration and gas heating in hot corona which develop around compact objects (see Sect. 3.6).

To finish, we also mention the betatron-magnetic pumping process (Parker 1958a). In this process, particles propagate along a magnetic field slowly varying with time. As the field strength increases, the particle momentum increases due to the conservation of the adiabatic invariant $p_{\perp}^{2} / B$. $^{2}$ If the particle suffers some scattering of its pitch-angle or some elastic collisions, a decrease of the magnetic field strength while keeping particle isotropization by pitch-angle scattering produces a net gain in energy during a magnetic field variation cycle.

2. Shock or shear-flow acceleration. One way to cure the inefficiency of the SFA is to allow scattering centers to have a mean direction of motion (Parker 1958a; Wentzel 1963). Then the mean relative energy gain scales as $\langle\Delta E / E\rangle \propto(U / v)$. This case occurs for a shock because of the advection of the scattering centers towards the shock front but also in the configuration of a shearing, for instance in jets. We do not explicitly for now discuss the case of shear-flow acceleration, the interested reader can refer to Rieger and Duffy (2006), and we consider hereafter the case of shock acceleration. Shear-flow acceleration will be reviewed in a forthcoming version of the text. The acceleration process is more efficient if particles can reside for a sufficient amount of time around the shock front (Kirk 1994). In fact, particle acceleration at shock waves covers three basic different processes (see Kirk 1994; Treumann and Jaroschek 2008b; Marcowith et al. 2016): diffusive shock

\footnotetext{
${ }^{1} U_{\mathrm{A}}=B / \sqrt{4 \pi \rho_{\mathrm{i}}}$, where $B, \rho_{\mathrm{i}}$ are respectively the local magnetic field strength, the ion mass density.

${ }^{2} p_{\perp}=p \sin \alpha$, where $\mathrm{p}$ and $\alpha$ are the particle momentum and pitch-angle (the angle between the particle velocity and magnetic field) respectively.
} 
acceleration (DSA), shock drift acceleration (SDA), shock surfing acceleration (SSA), all described below.

Before, let us introduce some elements of vocabulary associated with different descriptions of the shock front. First, particle acceleration requires the shock to be collisionless, i.e., to be mediated by electromagnetic processes rather than collisions otherwise collisions being the fastest process force the shocked particle distribution to be Maxwellian. This condition is usually fulfilled in astrophysical and space plasmas. At a macroscopic level a shock wave is characterized by a discontinuity in the thermodynamical variables of the flow. A shock occurs when the flow is supersonic, with a sonic Mach number $\mathcal{M}_{\mathrm{s}}=U_{\mathrm{sh}} / c_{\mathrm{s}}>1$, where $U_{\text {sh }}$ is the shock speed in the upstream medium restframe. Rankine-Hugoniot conditions give the jump in the flow density, velocity, pressure, temperature and entropy at the shock front (Treumann and Jaroschek 2008a). At a microscopic level a shock front is a complex, dynamical structure where multi-scale instabilities can develop (Marcowith et al. 2016). The so-called supercritical magnetized shock front is composed of three sub-structures: the foot, a bump in gas and magnetic field pressures due to the accumulation of ions reflected at the ramp, the ramp which marks the fast rise of the electromagnetic field potential and gas density and finally the overshoot-undershoot produced by the gyromotion of reflected ions moving in the post-shock gas. Shocks in astrophysics also include precursors in the upstream gas which can have very different origins: radiation, mixture of ions and neutrals or CRs. The size of these precursors make them usually impossible to explore numerically with the shock front structure as a single complex dynamical system.

As stated above particle acceleration in shocks proceeds through three different mechanisms. In DSA particles repeatedly gain energy by crossing the shock front back and forth (Drury 1983). SDA results from the effect of the convective electric field $\mathbf{E}=-\mathbf{U} / c \times \mathbf{B}$ upstream the shock front due to the motion of the flow at a speed U (Kirk 1994; Decker and Vlahos 1985). The particle guiding-center drifts due to the effect of the electric field and to the gradient of the magnetic field in the ramp. SSA results from the trapping of the particle at the shock front because of the combined effect of shock potential raise at the ramp and the convective upstream electric field (Sagdeev 1966). We will come back with more details on these mechanisms in Sects. 2.2 and 2.3.

3. Magnetic reconnection (REC). Magnetic reconnection is the process which transfers magnetic field energy into kinetic energy in an explosive event by re-arranging the magnetic field topology. The most simple 2D picture is sketched in the left panel of Fig. 1. Separatrices (green, dashed lines) divide the 2D plane into 4 different regions: in the left region, the magnetic field connects points $\mathrm{A}$ and $\mathrm{B}$. In the right region, the field connects points $\mathrm{A}^{\prime}$ and $\mathrm{B}^{\prime}$ and is oriented in opposite direction to the field in the left region. No field is present in the upper and lower region between the separatrices. In 2D, due to Ampère's law, a current pointing normal to the plane is necessarily present between the oppositely oriented fields. The REC process then leads to a re-arrangement of the field lines, lowering the magnetic energy. The field now connects the points A and A' (B and B' respectively). These field lines are highly bent and will relax, accelerating the plasma upwards and 

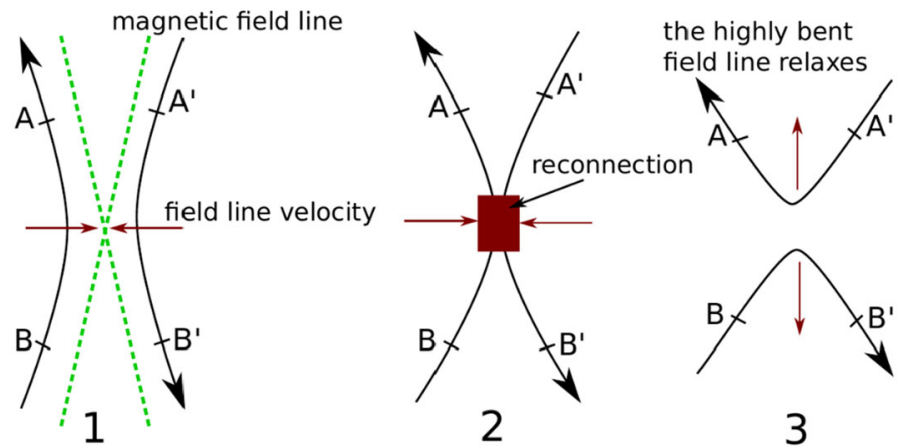

Fig. 1 The re-arrangement of the field topology in magnetic reconnection in a 2D model. Before the event (Image 1), points $\mathrm{A}$ and $\mathrm{B}\left(\mathrm{A}^{\prime}\right.$ and $\mathrm{B}^{\prime}$ respectively) are located on the same field line. After the event (3), field lines now are connecting points $\mathrm{A}$ and $\mathrm{A}^{\prime}$ (B and $\mathrm{B}^{\prime}$ respectively). Strongly accelerated outflow is driven in the directions where the highly bent magnetic field lines are relaxing. The dashed green lines are called separatrices, lines which separate the regions of field which are topologically not connected. Image adapted from Melzani (2014)

downwards. The term magnetic reconnection was first coined in Dungey (1958) and was later adopted by the community. More details about the REC process are provided in Sect. 2.5.

REC induces a transfer of magnetic energy into: heat, plasma and particle acceleration and hence radiation (Gonzalez and Parker 2016; Priest 1994). Particle acceleration in reconnection sites can either occur by a direct acceleration in electric fields in the current sheet, or because of Fermi first order acceleration in the plasma converging towards the reconnection zone or if particles are trapped in a contracting magnetic islands (de Gouveia Dal Pino and Kowal 2015). The physics of particle acceleration in kinetic reconnection is discussed in Sect. 4.2.

Acceleration mechanisms, as we have seen from the above rapid descriptions, intrinsically involve multi-scale processes which bring particles from the thermal to supra-thermal speeds. In astrophysics these processes have to explain the CR spectrum observed at Earth which extends at least over 15 orders of magnitude in energy (from $\mathrm{MeV}$ to $\mathrm{ZeV}$ ) and more than 30 orders of magnitude in flux (see Fig. 2). Note that in space plasmas, maximum energies reached by the energetic particles are more modest but still supra-thermal, and the particle distributions cover about 5 orders of magnitude (from keV to $\mathrm{GeV}$ ) (see, e.g., Zharkova et al. 2011 in the context of solar flares). The investigation of particle acceleration then requires different numerical approaches to probe the different inter-connected scales involved in the process of acceleration. Multiple techniques are also required as actually it is not possible to account for such large dynamical spatial, time and energy scales even with modern computers. It is the main object of this review to address these different techniques.

\subsection{Layout}

This review is organized as follows. Section 2 addresses the scientific context. It describes the main acceleration processes at work in astrophysical plasma systems. 


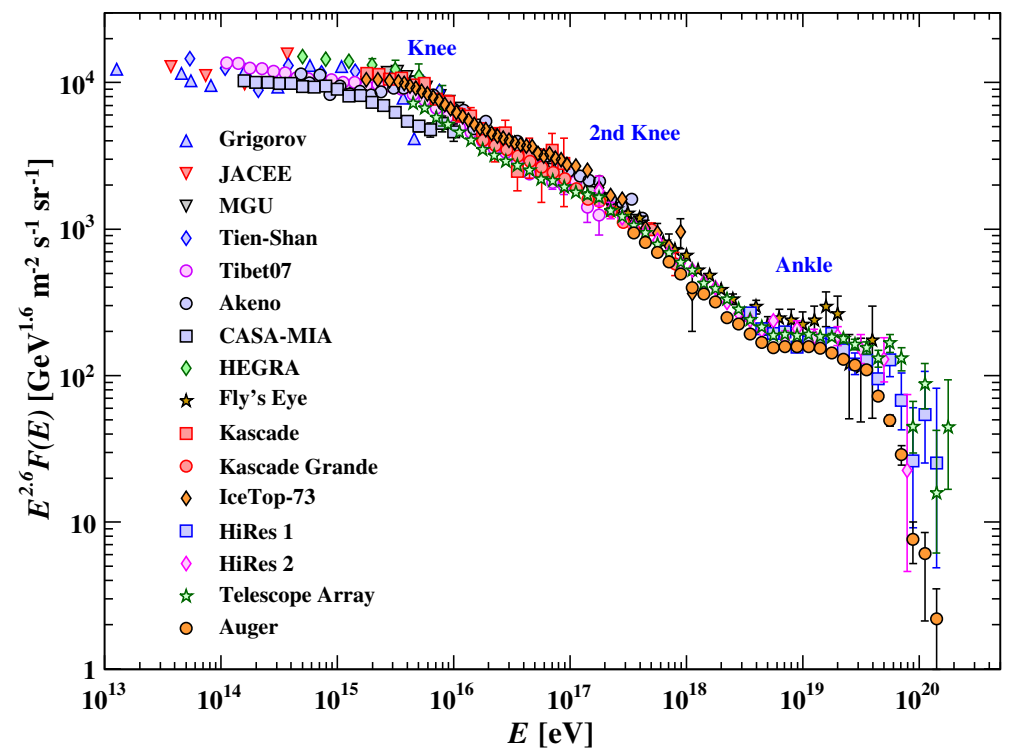

Fig. 2 The cosmic ray spectrum observed at the Earth multiplied by $E^{2.6}$. Image reproduced with permission from Patrignani and Particle Data Group (2016), copyright by Regents of the University of California

It also a short review on the on-going experimental efforts to reproduce collisionless shocks, magnetic reconnection and particle acceleration in laser-plasma-based experiences. The next sections treat the different numerical approaches to investigate particle acceleration from microscopic scales to macroscopic scales. Section 3 describes the different numerical methods adapted to the description of plasma kinetics. Section 4 discusses particle acceleration and transport at micro- and meso-plasma scales. Section 5 describe numerical techniques developed to follow macroscale dynamics and detail recent results on particle acceleration and transport in astrophysical flows. We conclude in Sect. 6.

How to read this review The scientific questions and the numerical experiments developed to investigate them are entangled. We have decided to describe this complex modelling in two steps. The first step presents a general description of the main acceleration processes in astrophysical plasmas. This presentation is the main purpose of Sect. 2. Notice that we complement it by a dedicated section addressing some recent studies of particle acceleration at collisionless shocks and magnetic reconnection in the context of laser plasmas in Sect. 2.6. The second step describes technical numerical aspects. They are presented in Sects. 3.4 to 3.5 and in all sub-sections of Sect. 5. Sections 3, 4, 5 then include discussions which connect the numerical work and scientific questions exposed in Sect. 2.

\subsection{List of acronyms and notations}

All quantities are in cgs Gaussian units. 


\begin{tabular}{ll}
\hline Acronym name & Definition \\
\hline AGN & Active galactic nucleus \\
AMR & Adaptive mesh refinement \\
CFL & Courant-Friedrichs-Lewy \\
CR & Cosmic Ray \\
DCE & Diffusion-convection equation \\
DSA & Diffusive shock acceleration \\
EP & Energetic particle \\
FDM & Finite difference method \\
FVM & Finite volume method \\
FP & Fokker-Planck \\
GRB & Gamma-ray burst \\
HD & Hydrodynamics \\
ISM & Interstellar medium \\
MFA & Magnetic field amplification \\
MHD & Magneto-hydrodynamics \\
NLDSA & Non-linear diffusive shock acceleration \\
PDE & Partial differential equation \\
PIC & Particle-in-cell \\
PWN & Pulsar wind nebula \\
REC & Magnetic reconnection \\
SDA & Shock drift acceleration \\
SFA & Stochastic Fermi acceleration \\
SNR & Supernova remnant \\
SSA & Shock surfing acceleration \\
\hline & \\
\hline
\end{tabular}

We recall here the definition of the different quantities used to construct the main parameters involved in shock acceleration processes: $B$ is the magnetic field strength, $\rho$ is the gas mass density, $\rho_{\mathrm{i}}$ is the ion mass density, $\gamma_{\mathrm{ad}}$ is the gas adiabatic index, $v$ and $p$ are the charged particle speed and momentum and $Z e$ is the charge.

\begin{tabular}{ll}
\hline Notation & Definition \\
\hline$\theta_{\mathrm{B}}$ & $\begin{array}{l}\text { Shock magnetic field obliquity } \\
\text { (Angle between field lines and shock normal) }\end{array}$ \\
$U_{\mathrm{sh}}$ & Shock velocity in the upstream (observer)frame \\
$U_{\mathrm{A}}=B / \sqrt{4 \pi \rho_{\mathrm{i}}}$ & Local Alfvén speed \\
$M_{\mathrm{A}}=U_{\mathrm{sh}} / U_{\mathrm{A}}$ & Alfvénic Mach number \\
$c_{\mathrm{S}}=\sqrt{\gamma_{\mathrm{ad}} P / \rho}$ & Local sound speed \\
$M_{\mathrm{S}}=U_{\mathrm{sh}} / c_{\mathrm{S}}$ & Sonic Mach number \\
$\sigma=B^{2} / 4 \pi \rho c^{2}$ & Local magnetization \\
$\sigma=\omega_{\mathrm{ci}}^{2} / \omega_{\mathrm{pi}}^{2}$ & (Ratio of the upstream magnetic pressure to the upstream gas kinetic energy) (shocks) \\
$\beta_{\mathrm{p}}$ & Local magnetization \\
$r_{\mathrm{L}}=p v / Z e B$ & (Ratio of the square of cyclotron to plasma frequencies) (reconnection) \\
\hline
\end{tabular}

Notice of caution: There is not a unique way to define the magnetization parameter $\sigma$. In shock studies, $\sigma$ is the ratio of the magnetic pressure to the kinetic energy of the ambient gas, all quantities being measured in the upstream rest-frame. In relativistic 
shock studies, the magnetization parameter is sometimes defined as $\sigma=B^{2} / 4 \pi \Gamma U c^{2}$, where $\Gamma=\sqrt{1+U^{2}}$ is the Lorentz factor of the shock and $U$ is the four-velocity of the flow (Marcowith et al. 2016). In reconnection studies, $\sigma$ is the ratio of the square of cyclotron to plasma frequencies. It is related to the the ratio $r_{\mathrm{A}}=U_{\mathrm{A}} / c$ by $\sigma=r_{\mathrm{a}}^{2} /\left(1-r_{\mathrm{A}}^{2}\right)($ e.g., Sironi and Beloborodov 2019).

\section{Astrophysical and physical contexts}

Following the introductory remarks in Sect. 1, we present below an overview of the astrophysical and physical contexts where the numerical tools discussed in this review are actively developed. We aim here at a short description of the basic concepts necessary to describe particle acceleration. In particular, we show that particle acceleration involves a large range in scale/time/energy which justifies the use of very different numerical techniques detailed in the next sections.

First, in Sect. 2.1 we briefly overview the mechanism of stochastic acceleration (SA). Then the three next sections cover different aspects of the physics of particle acceleration at collisionless shocks. In Sect. 2.2 we provide a general and rather detailed presentation of the physics of diffusive shock acceleration (DSA) which is one of the main frameworks to study particle acceleration in astrophysical systems. Beyond a standard description of the process itself we discuss specific issues connected with the acceleration of cosmic rays (CRs) at fast astrophysical shock waves: the injection problem and non-linear back-reaction of CRs over the flow solution. These two difficulties require the development of specific scale-dependent numerical techniques described in the next sections. Section 2.3 is a short presentation of the other two shock acceleration processes, namely the shock drift acceleration (SDA) and the shock surfing acceleration (SSA) which are especially relevant for particle injection in the DSA process. Section 2.4 discusses the specific case of Fermi acceleration at relativistic shocks and the development of micro turbulence at these shock fronts. Magnetic reconnection (REC) is discussed in some detail in Sect. 2.5, where we present the most relevant vocabulary necessary to understand particle acceleration in reconnection structures. Section 2.6 reviews the most important undergoing or planned laser experiments to study particle acceleration. This rapidly growing field of research starts now to investigate astrophysically relevant conditions for particle acceleration at collisionless shocks and magnetic reconnection. Notice that we decided to not include any review of acceleration processes in space plasmas, this will deserve a special section in a forthcoming version.

It should be stressed that by no means this section is intended to be exhaustive. It has to be understood as a short introduction to the scientific cases where the different simulation techniques discussed hereafter are developed. For each type of acceleration/transport mechanism we refer the interested reader to more complete dedicated reviews. 


\subsection{Stochastic acceleration}

As discussed in Sect. 2.2.1 stochastic acceleration occurs because, on average, energetic particles at a speed $\mathrm{v}$ interact with scattering centers moving at a speed $U$ more often through head-on collisions than through rear-on collisions if $v \gg U$. This results in a broadening of the particle distribution and an increase of the mean particle energy (Melrose 1980). In astrophysical plasmas the scattering centers often ${ }^{3}$ can be described as plasma waves, and when we deal with high-energy CRs these waves can be described using the MHD approximation (Parker 1955; Sturrock 1966; Kulsrud and Ferrari 1971). But it is necessary to go beyond MHD if we want to consider the acceleration of non-relativistic or mildly relativistic particles (Marcowith et al. 1997; Pryadko and Petrosian 1998).

As explained in Sect. 1, well-known caveats prevent the interpretation of the CR spectrum observed at the Earth as resulting from stochastic acceleration by MHD waves: (1) the non-universality of the distribution of accelerated particles, (2) a weak relative energy gain at each wave-particle interaction scaling as $(U / v)^{2}$. The second issue can be partly overcome if we consider the case of low energy (sub-GeV) CR propagation in the ISM, as in that case the ratio v/U drops. Still, an important problem results in the prohibitive amount of ISM turbulence necessary to re-accelerate the low energy end of CR spectrum (Ptuskin et al. 2006; Thornbury and Drury 2014; Drury and Strong 2017). Nevertheless, SA has been invoked to be an important source of turbulence damping and particle acceleration in solar flares (Petrosian 2012), in active corona above the accretion discs around compact objects (Dermer et al. 1996; Liu et al. 2004; Belmont et al. 2008; Vurm and Poutanen 2009), in SNRs or their associated superbubbles (Bykov and Fleishman 1992; Kirk et al. 1996; Marcowith and Casse 2010; Ferrand and Marcowith 2010), in galaxy clusters (Brunetti and Lazarian 2007), or in the case the wave phase (Alfvén) speed gets close to the speed of light as can be the case in AGNs (Henri et al. 1999), in GRBs (Schlickeiser and Dermer 2000), or in pulsar winds (Bykov et al. 2012).

\subsection{Diffusive shock acceleration}

DSA is probably the favored production mechanism of CRs. It is thought to be a natural outcome of collisionless shocks, and so is believed to be at work in astrophysical shocks at all scales, active in the bow shocks in the solar system, in the blast waves of supernova remnants (SNRs) or in the internal shocks in the jets of gamma-ray bursts (GRBs) or active galactic nuclei (AGNs). DSA is rooted in the early ideas of Fermi $(1949,1954)$; it was developed independently in the late 1970s by Krymskii (1977), Axford et al. (1977), Bell (1978a, b), Blandford and Ostriker (1978), see Drury (1983), Jones and Ellison (1991), Malkov and Drury (2001) for comprehensive reviews. A key feature of DSA is that it produces power-law distributions as a function of energy (although this

\footnotetext{
3 Note however that, similarly to Fermi's original ideas, some models invoke finite amplitude waves or shocks as the origin of stochastic energy exchanges with energetic particles, see in different astrophysical contexts e.g. Bykov and Toptygin (1987), Achterberg (1990), Pelletier and Marcowith (1998), Gialis and Pelletier (2004).
} 
spectrum can be altered by non-linear effects), which is similar to the CR spectrum as observed from the Earth, modulated by CR transport and escape from the Milky Way. DSA requires two ingredients to accelerate particles: a converging flow (the shock wave), and scattering centers (perturbations of the magnetic field). In this mechanism individual microscopic particles can be accelerated up to very high energies because they are interacting a large number of times with the macroscopic shock discontinuity before escaping the system. Again, one major difficulty in simulating this process becomes apparent: DSA is intrinsically a multi-scale problem.

\subsubsection{Fermi processes and building power-laws}

In his original model, Fermi considered the interaction of charged particles with moving magnetized clouds. In the cloud frame, the particle (of velocity v) is elastically deflected around the $\mathbf{B}$ field. In the Galactic frame (with respect to which the cloud is moving at velocity $\mathbf{U}$ ), the energy $E$ of the particle changes according to

$$
\frac{\Delta E}{E}=-2 \frac{\mathbf{v} \cdot \mathbf{U}}{c^{2}} .
$$

The effect depends on the geometry of the encounter: for head-on collisions $(\mathbf{v} \cdot \mathbf{U}<0)$ the particle gains energy $(\Delta E>0)$, whereas for overtaking collisions $(\mathbf{v} \cdot \mathbf{U}>0)$ it loses energy $(\Delta E<0)$. The exchange is mediated by the magnetic field, even though $\mathbf{B}$ does not appear in the formula ( $\Delta E$ is nothing but the work of the Lorentz force exerted on the particle by the electric field $\mathbf{E}$ induced by the moving $\mathbf{B}$ ). For a random distribution of moving clouds, after many interactions the particle experiences a net energy gain, because head-on collisions are more likely. This is only an average gain, hence the name stochastic acceleration, and it scales as $\beta^{2}$ where $\beta=v / c$, hence the name second-order Fermi acceleration (or simply Fermi II). Fermi himself realized that this process was probably not efficient enough to produce the bulk of Galactic CRs. Now if somehow only face-on collisions occur, then the energy gain is systematic, hence the name regular acceleration, and it scales as $\beta$, hence the name first-order Fermi acceleration (or simply Fermi I).

A shock wave (the $\mathrm{S}$ in DSA) provides such a configuration: both the upstream and the downstream medium see the opposite side arriving at the same speed $\Delta U=$ $\frac{r-1}{r} V_{\mathrm{sh}}\left(=\frac{3}{4} U_{\mathrm{sh}}\right.$ if $\left.r=4\right)$ where $r$ is the compression ratio and $U_{\mathrm{sh}}$ is the speed of the shock (with respect to the unperturbed upstream medium). Let us further assume that magnetic turbulence in the vicinity of the shock efficiently scatters off the particles (leading to the D in DSA), so that they are effectively isotropized on each side of the shock, meaning that their mean velocity follows the local flow velocity. ${ }^{4}$ Then, the particles experience a regular Fermi acceleration, the clouds being replaced by a reflecting wall moving at velocity $\Delta U$ Averaging Eq. (1) over all angles, one gets a mean energy gain

\footnotetext{
4 More precisely, the velocity that matters for particles is that of the magnetized waves present in the flow, the difference with respect to the fluid flow is called the Alfvén drift, in the case the main scattering waves are Alfvén waves, see Sects. 5.3 and 5.7.2.
} 


$$
\left\langle\frac{\Delta E}{E}\right\rangle=\frac{4}{3} \frac{\Delta U}{c}=\frac{4}{3} \frac{r-1}{r} \frac{U_{\text {sh }}}{c}\left(=\beta_{s} \text { if } r=4\right) .
$$

By considering the duration of a complete reflection from the opposite medium, and the probability of escape from the acceleration region, one can derive the final distribution in energy of particles. To do so, two approaches are possible: a microscopic approach, where one considers the fate of individual particles, and a kinetic approach, where one reasons with their distribution function as a function of energy or rather momentum (see details of the calculations for both approaches in Drury (1983) and references therein). This basic choice will also apply to the numerical methods presented in this review. From a general point of view an acceleration mechanism can be characterized by its acceleration time $\tau_{\text {acc }}$ (defined so that particles are accelerated at a rate $\partial E / \partial t=$ $E / \tau_{\text {acc }}$ ) and its escape time $\tau_{\text {esc }}$ (defined so that particles escape the accelerator at a rate $\left.\partial N / \partial t=N / \tau_{\text {esc }}\right)$. If particles are injected at an energy $E_{0}$ with a rate $Q\left(E_{0}\right)$, after a time $\mathrm{t}$ a number density $N(E) d E=Q\left(E_{0}\right) \exp \left(-t(E) / \tau_{\mathrm{esc}}\right) d t$ of particles will have escaped. Now energy and time are linked by $t(E)=\tau_{\text {acc }} \ln \left(E / E_{0}\right)$, inserting this time in the previous relation leads to the steady-state solution

$$
N(E) \propto E^{-s} \text { with } s=1+\frac{\tau_{\mathrm{acc}}}{\tau_{\mathrm{esc}}} .
$$

In the limit where escape never occurs $\left(\tau_{\text {esc }}=\infty\right)$, the hardest spectrum one can obtain is $N(E) \propto E^{-1}$. In DSA the ratio $\tau_{\text {acc }} / \tau_{\text {esc }}$ turns out to be independent of $E$, and so one gets a power-law distribution, of index

$$
s=\frac{r+2}{r-1}(=2 \text { if } r=4) \text {. }
$$

The spectral index is controlled by the compression ratio of the shock, and so is a universal value for strong shocks.

\subsubsection{The transport equation and the diffusion coefficient}

Assuming the particle distribution is isotropic (to first order) in momentum $\mathbf{p}$, and considering here for simplicity a plane-parallel shock along direction $x$, in the kinetic description we may work with the quantity $f=f(x, p, t)$, which is defined so that the number density is $n(x, t)=\int f(x, p, t) 4 \pi p^{2} \mathrm{dp}$, and which obeys the convection-diffusion equation $(\mathrm{CDE})^{5}$ :

$$
\frac{\partial f}{\partial t}+\frac{\partial(U f)}{\partial x}=\frac{\partial}{\partial x}\left(D \frac{\partial f}{\partial x}\right)+\frac{1}{3 p^{2}} \frac{\partial p^{3} f}{\partial p} \frac{\partial U}{\partial x} .
$$

On the right-hand side of the equation, the second term represents advection in momentum, powered by the fluid velocity divergence $\partial U / \partial x$, while the first term models the

\footnotetext{
5 This equation is obtained by averaging the full Fokker-Plank equation (FPE) over particle pitch-angle and azimuthal gyromotion angle (see Sect. 3.6).
} 
spatial diffusion of the particles, resulting from their scattering off magnetic waves, and described by a diffusion coefficient $D(x, p)$. This coefficient, together with the shock speed $U_{\mathrm{sh}}$, sets the space- and time-scales of DSA. Upstream of the shock front, particles can counter-stream the flow up to a distance

$$
\ell_{\text {prec }}(p)=\frac{D(p)}{U_{\text {sh }}}
$$

which sets the scale of what is called the CR precursor region. The acceleration timescale, defined so that $d p / d t=p / t_{\mathrm{acc}}$, goes as

$$
t_{\mathrm{acc}}(p) \propto \frac{D(p)}{U_{\mathrm{sh}}^{2}}
$$

where the proportionality factor is of order 8-20 depending on the shock obliquity and the Rankine-Hugoniot conditions linking up- and downstream magnetic field strengths, see Reynolds (1998). For DSA to work requires $\ell_{\text {prec }}$ to be less than the accelerator's size, and $t_{\text {acc }}$ to be less than the accelerator's age, which puts limits on the maximum momentum $p_{\max }$ that the particles can reach (for particles that radiate efficiently like electrons, $p_{\max }$ may also be limited by losses). The diffusion law is thus a critical ingredient in DSA. This aspect will however be treated in very different ways in different kinds of numerical simulations. In kinetic approaches, where one solves one equation of the kind of Eq. (5), the diffusion coefficient $D(x, p)$ or some equivalent quantity must be specified. In microscopic approaches, where one directly integrates the equation of motion of individual particles, the diffusion coefficient may actually be measured from the observed paths of an ensemble of particles.

Computing the value of the diffusion coefficient from theory is a difficult problem (see Shalchi 2009 b for a review). Very generally, the diffusion coefficient can be expressed as $D=\frac{1}{3} \ell . v$ where again $v$ is the particle velocity and $\ell$ its mean free path. When charged particles are deflected by Alfvén waves, $\ell$ is inversely proportional to the energy density $\delta B^{2}$ in waves present with the resonant wavelength $\lambda \simeq r_{\mathrm{g}}$, where $r_{\mathrm{g}}=\frac{p c}{q B}$ is the particle gyroradius. A special case of interest is the so-called "Bohm limit" (see e.g. Kang and Jones 1991; Berezhko and Völk 1997; Bell 2013) reached when $\ell \simeq r_{\mathrm{g}}$, that is when the particles are scattered within one gyroperiod, meaning that the turbulence is random $(\delta B \sim B)$ on the scale $r_{\mathrm{g}}$. This constitutes a lower limit on the value of the (parallel) diffusion coefficient, and so on the acceleration time-scales. In that case, $D \propto p v$ so that

$$
D_{B}(p)=D_{0} \frac{p^{2}}{\sqrt{\left(1+p^{2}\right)}}
$$

where one can evaluate $D_{0} \simeq 3 \times 10^{22} / B \mathrm{~cm}^{2} \cdot \mathrm{s}^{-1}$ with $B$ in $\mu G$, and $p$ is expressed in $m_{p} c$ units.

Historically the Bohm limit has been widely favored in the literature, in its true form in Eq. (8) or with a free normalization and only keeping the "Bohm scaling" in $p$, and using the exact dependence on $p$, or only the relativistic scaling $D(p) \propto p$, 
or a parametrized scaling of the form $D(p)=D_{0} p^{\alpha}$ with free index $\alpha$ (commonly informally called "Bohm-like" coefficients). This choice of the Bohm limit may be in part due to the fact that it is the most favorable case, and also may just stem from habit given the lack of clear theoretical alternatives. Analytically it has only been derived under the assumption of strong turbulence Shalchi (2009a), and numerical studies have found that it does not generally hold (Casse et al. 2002; Candia and Roulet 2004; Parizot 2004). The validity of this assumption in the context of supernova remnant studies has been regularly questioned (Kirk and Dendy 2001; Parizot et al. 2006), and in the context of interplanetary shocks other models have been used according to the turbulence properties and shock obliquity (Dosch and Shalchi 2010; Li et al. 2012). In any case, when it comes to simulations, a key aspect is how strongly the diffusion coefficient depends on the particle's energy, given relations (6) and (7).

\subsubsection{The injection problem}

DSA is a bottom-up acceleration mechanism, whereby (a fraction of) the particles from a plasma get boosted to very large energies. The particles are accelerated from a non-thermal distribution that extends beyond the thermal distribution of the plasma (often assumed to be a Maxwellian). The way these two populations are connected is a delicate problem. The discussion of DSA above assumes that particles are sufficiently energetic that they can leap over the shock wave and perceive it as a discontinuity, meaning that their mean free path in the magnetic turbulence is already larger than the physical width of the shock wave, which is typically of the order of a few Larmor radii of thermal ions. The way by which particles from the background plasma enter the acceleration process is known as the "injection" mechanism. The general idea, referred to as "thermal leakage" is that particles heated at the shock may be able to re-cross the shock to start the DSA cycle (Malkov and Voelk 1995; Malkov 1998). The efficiency of the injection determines the fraction of the available shock energy that is channeled into energetic particles. It is widely expected to vary as a function of parameters such as the shock obliquity, although there is no firm agreement yet on what are the most favorable configurations.

Injection is treated in much different ways according to the level of the numerical modeling. In kinetic approaches that decouple the non-thermal population (obeying Eq. 5) and the thermal population (obeying classical conservation laws), the injection process is parametrized. The simplest way to do this is to postulate that some fraction $\eta$ of the particles crossing the shock enter the acceleration process, at some momentum $p_{\text {inj }}$ above the typical thermal momentum. The requirement that the particles power-law matches the plasma Maxwellian at $p_{\text {inj }}$ actually implies that these quantities are related, as shown in Blasi et al. (2005). A more advanced approach is to use a "transparency function" to inject particles at the shock, as done by Gieseler et al. (2000). In contrast, in the Monte-Carlo simulations of the kind of Ellison and Eichler (1984) no formal distinction is made between the thermal and non-thermal populations, allowing for a more consistent treatment of injection, for a given scattering law. Only PIC simulations are able to directly address the formation of the collisionless shock concomitantly with the energization of particles, and it has been only very recently that the computational power has become sufficient to see the DSA 


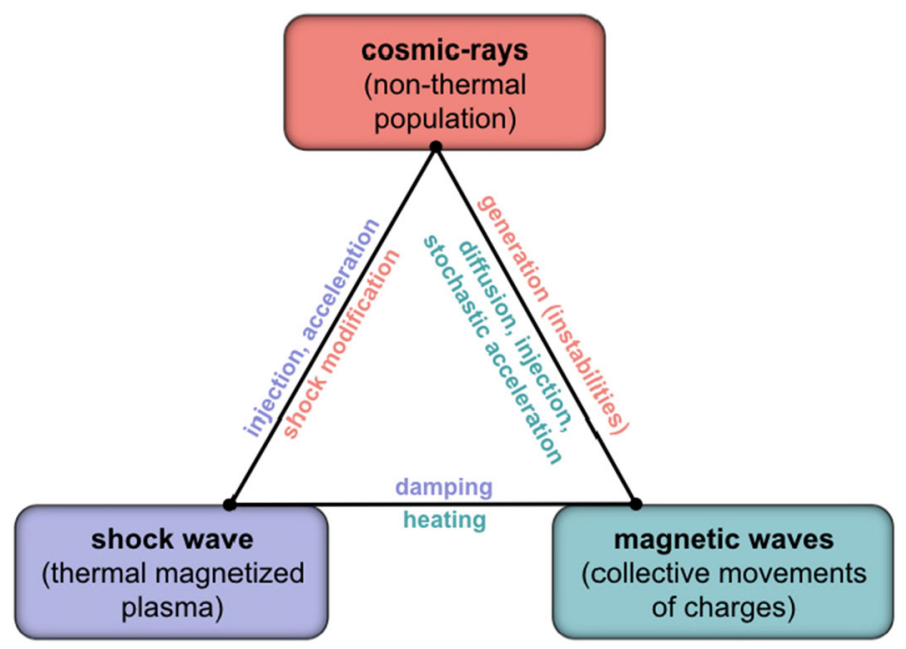

Fig. 3 Sketch of the physical components and their couplings in the diffusive shock acceleration mechanism

power-law naturally emerge (e.g., Caprioli and Spitkovsky 2014a)—although still on very small space- and time-scales compared to any astrophysical object of interest. The results associated to these simulations are described with more details in Sect. 4. This again illustrates the need for a model at different scales and their entanglement, a given numerical approach often relying on results obtained from other approaches for the aspects it cannot describe.

\subsubsection{Back-reaction and non-linear effects}

DSA at astrophysical shocks involves three kinds of actors: energetic particles, a plasma flow, and magnetic waves (see Fig. 3). Charged particles are being injected from the plasma and accelerated at the shock, thanks to their confinement by magnetic turbulence. In our discussion so far we have assumed a prescribed background plasma flow and magnetic turbulence, that is, we were implicitly discussing the "test particle" regime. But if the acceleration process is efficient (meaning that a substantial fraction of the available energy ends up into particles), then the particles will play a role in the dynamics of the plasma and in the evolution of the magnetic field. This in turn will affect the way they are being accelerated, so that the DSA process becomes non-linear (NLDSA). The time-dependent problem is intractable analytically in the general case, which is the reason why studies of (efficient) particle acceleration rely on numerical techniques, as described in this review. To end this section on DSA, we summarize the main aspects of the two back-reaction loops: of the particles on the plasma flow, and of the particles on the magnetic turbulence.

Even before DSA theory was established, Parker (1958b) noted that CRs modify the medium in which they propagate: being relativistic they lower the overall adiabatic index of the flow. We can make this more precise by considering the CR pressure, defined as 


$$
P_{\mathrm{cr}}=\int_{p} \frac{p v}{3} f(p) 4 \pi p^{2} d p=\frac{4 \pi}{3} m_{p} c^{2} \int_{p} \frac{p^{4}}{\sqrt{1+p^{2}}} f(p) d p
$$

(where in the right expression momenta are expressed in $m_{p} c$ units). This quantity can grow without limit in the linear regime. But as CRs diffuse upstream of the shock in an energy dependent way, a gradient of CR pressure forms in the precursor, which produces a force acting on the plasma. This CR-induced force pre-accelerates the plasma ahead of the shock front, leading to the formation of a smooth, spatially extended velocity ramp upstream of the shock front. The shock itself is thus progressively reduced to a so-called "subshock", whose compression ratio is $r_{\text {sub }}<4$, while the overall compression ratio $r_{\text {tot }}$ (measured from far upstream to far downstream) becomes $>4$ from mass conservation - the plasma is more compressible when particles become relativistic, and even more so when the particles escape the system (Berezhko and Ellison 1999). As particle of different energies can explore regions of different extent ahead of the shock, they will feel different velocity jumps, so that the spectral slope defined by Eq. 4 becomes energy-dependent. The spectrum is thus no longer the canonical power-law, but gets concave. Particles of low energy $\left(p \ll m_{p} c\right)$ only sample the sub-shock, feeling a compression $r_{\text {sub }}$ that produces a slope larger than the canonical value of 2 (that is, a steeper spectrum), whereas particles at the highest energies sample the whole shock structure, feeling a compression $r_{\text {tot }}$ that produces a slope that is smaller (that is, a flatter spectrum). So one of the historically most attractive features of DSA - its ability to naturally produce power-law spectra-cannot strictly hold when it is efficient.

Turning to magnetic turbulence, it was also observed early on (e.g., Skilling 1975b) that the CRs can generate themselves the waves that will scatter them off. They can indeed trigger various instabilities by streaming upstream of the shock, which generates magnetic turbulence, which is then advected to the shock front and the downstream region. Denoting by $W(\mathbf{k}, x, t)$ the power spectrum of the magnetic waves where $\mathbf{k}$ is the wave vector, its evolution obeys a transport equation of the general form (here written for simplicity along one dimension). Assuming for simplicity that $\mathrm{U}$ is constant we have:

$$
\frac{\partial W}{\partial t}+U \frac{\partial W}{\partial x}=\Gamma_{g}-\Gamma_{d}
$$

where $\Gamma_{g}$ is the growth rate of the waves, which is dictated by the particles, and $\Gamma_{d}$ is their damping rate in the plasma. For a CR-induced streaming instability the growth rate $\Gamma_{g}$ scales as the gradient in the CR pressure. Using hybrid MHD+particle simulations, Lucek and Bell (2000), Bell (2004) showed that the seed magnetic field can be amplified by up to two orders of magnitude, which is important because in turn the magnetic field controls the diffusion and confinement of the particles and thus the maximum energy that they can reach (Bell and Lucek 2001). This discovery prompted a slew of work on this complex topic (see Schure et al. 2012 for a review). Two regimes of streaming instabilities can operate, a resonant instability at work when the Larmor radius of a particle matches the wavelength of a perturbation, and a non-resonant instability driven by the current of particles (see also Gary 1991). The amplified field saturates at an energy density $\delta \mathbf{B}^{2}$ that scales as $U_{\text {sh }}^{2}$, or possibly even $U_{\text {sh }}^{3}$ (Vink 2012). Other longer wavelength instabilities can be triggered too. Combined with 


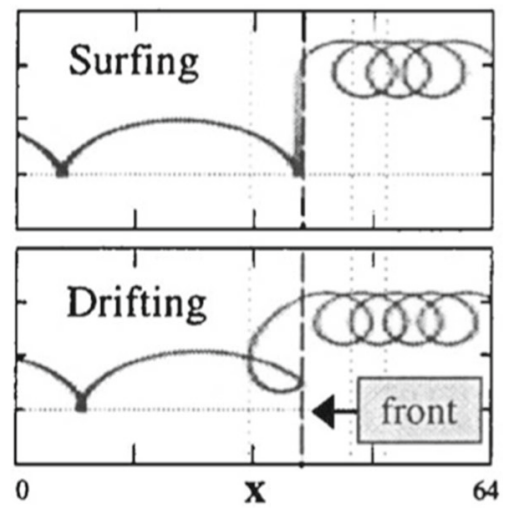

Fig. 4 Particle trajectories along the shock front due to SDA or SSA mechanisms. The flow is directed along the $x$-direction, and particles are injected at the left of the box. The magnetic field is perpendicular to the plane of the plot, as revealed by the gyro-motion of the particles. In the SDA process, the particle is forced to cross the shock several times. In the SSA process, the particle moves along the shock front. Image reproduced with permission from Shapiro and Üçer (2003). Copyright by Elsevier

observational evidence for high magnetic fields at the shocks of young supernova remnants (Reynolds et al. 2012), this has lead to the current view that magnetic field amplification (MFA) is a critical ingredient of DSA. This effect has been integrated in numerical simulations in different ways depending on the level of description of the particles and waves. For instance, in the kinetic description of DSA, one can in principle compute the diffusion coefficient $D(x, p, t)$ self-consistently from the cosmic-rays distribution $f(x, p, t)$. Obtaining a fully consistent description of the dynamical evolution of the particles, the plasma flow, and the magnetic turbulence, is still a work in progress.

\subsection{Shock drift and shock surfing acceleration}

These two acceleration processes rely on the effect of the convective electric field $\mathbf{E}=-\mathbf{U} / c \times \mathbf{B}$ induced by magnetized fluid motions towards the shock. The difference between the two processes results from the way the particles are either confined at the shock front in the case of shock surfing or move up- and downstream in the case of shock drift (Hudson 1965). Figure 4 shows different trajectories adopted by particles due to these two different processes.

\subsubsection{Shock drift acceleration: SDA}

Acceleration associated to the drift of the particle's guiding center depends strongly on whether the shock is super- or subluminal. The super- or subluminal character of a shock depends on the speed of the intersection point of the upstream magnetic field with the shock front: in superluminal shocks this speed is larger than the speed of light, in subluminal shocks it is smaller. 
In subluminal shocks it is always possible to find a frame where the convective electric field vanishes (so where the fluid velocity lies along the magnetic field line direction), this is the so-called de Hoffmann-Teller (HT) frame (Kirk 1994). In the HT frame particle energy is conserved. As an upstream field line intersects the shock the particle guiding center drifts along the shock and can either be transmitted or reflected at the shock front because the magnetic field is compressed there. The energy gain is the highest for particles reflected at the shock front (Decker 1988). This effect is similar to a reflection at the edge of a magnetic bottle. A calculation assuming that adiabatic theory applies uses a Lorentz transformation between a frame at which the shock is stationary to the HT frame to derive the energy gained by a particle reflected at the shock. Averaging over initial particle pitch-angles gives a ratio of the particle energy after the reflection to the initial energy of

$$
\left\langle\frac{E_{\mathrm{f}}}{E_{\mathrm{i}}}\right\rangle=\frac{1+\sqrt{1-b}}{1-\sqrt{1-b}},
$$

where $b=B_{\mathrm{u}} / B_{\mathrm{d}}$ is the ratio of the upstream to downstream magnetic field strengths. For $b=0.25$ (for a compression of the magnetic field by a factor 4 ) we find a maximum ratio $\left\langle E_{\mathrm{f}} / E_{\mathrm{i}}\right\rangle_{\max } \simeq 15.8$ and only half of this for a transmitted particle.

In superluminal shocks, a configuration obtained in perpendicular shocks, the adiabatic invariant $p_{\perp}^{2} / B$ is conserved even while the particle crosses the shock, as long as its speed is much larger than the shock speed (Webb et al. 1983; Whipple et al. 1986). The maximum value of the ratio $E_{\mathrm{f}} / E_{\mathrm{i}}$ is $1 / \sqrt{b}=2$. Superluminal shocks are the rule in relativistic flows. ${ }^{6}$ Begelman and Kirk (1990) investigate the condition under which SDA process operates at relativistic perpendicular shocks associated with the synchrotron emission of radio galaxy hot spots. As the flow speed gets closer to the speed of light the condition for the adiabatic theory breaks down. Begelman and Kirk (1990) propose an alternative method by following individual particle orbits. Due to shock dynamics, particles can cross the shock front at maximum three times before being advected downstream.

\subsubsection{Shock surfing acceleration: SSA}

Shock surfing is produced when a particle is trapped between the shock electrostatic potential $e \phi(+e$ is the particle charge in case of a proton) which appears at the shock ramp and the upstream Lorentz force along the shock normal which carries the particle back to the front. Original ideas about this mechanism can be found in Sagdeev (1966), Sagdeev and Shapiro (1973). The particle is accelerated under the action of the convective electric field until its kinetic energy along the shock normal exceeds $e \phi$. The trapped particle accelerates essentially along the shock front like a surfer's motion along a wave; hence Katsouleas and Dawson (1983) name this process shock surfing acceleration. The SSA mechanism is often invoked at quasi-perpendicular shocks as a pre-acceleration process. It allows to inject particles beyond the energy threshold

\footnotetext{
${ }^{6}$ The shock is subluminal only if the obliquity angle between the upstream magnetic field and the shock normal is $\theta_{\mathrm{u}} \leq 1 / \Gamma_{\mathrm{sh}}$, where $\Gamma_{\mathrm{sh}}$ is the shock Lorentz factor.
} 
for DSA to operate (Zank et al. 1996; Lee et al. 1996). Particles gain energy as long as they stay trapped at the shock front. The acceleration ceases if they escape either upstream if the magnetic field has some obliquity or downstream if the Lorentz force exceeds the electrostatic force at the shock layer. Particle acceleration can operate also in the relativistic regime where particles with small initial speeds are trapped the longest. Shapiro and Üçer (2003) for instance find that particles can have 10 bounces and reach Lorentz factors $\sim 2$ through this mechanism.

\subsection{Fermi acceleration process at relativistic shocks}

We now discuss how particle acceleration is affected when the flow itself is relativistic.

\subsubsection{General statements}

If we consider a relativistic shock front moving with a Lorentz factor $\Gamma_{\text {sh }}=$ $1 / \sqrt{\left(1-\left(U_{\mathrm{sh}} / c\right)^{2}\right)}$, the relative energy gain as the particle is doing a shock crossing cycle (e.g., up-down-upstream) can be obtained from relativistic kinematics by imposing a double Lorentz transformation between the upstream and downstream rest frames. The relative energy variation between the final $E_{\mathrm{f}}$ and the initial $E_{\mathrm{i}}$ particle energies is (Gallant and Achterberg 1999)

$$
\frac{\Delta E}{E}=\frac{\left(E_{\mathrm{f}}-E_{\mathrm{i}}\right)}{E_{\mathrm{i}}}=\Gamma_{\mathrm{r}}^{2}\left(1-\beta_{\mathrm{r}} \mu_{u \rightarrow d}\right)\left(1+\beta_{\mathrm{r}} \mu_{d \rightarrow u}^{\prime}\right)-1
$$

where unprimed and primed quantities mark upstream and downstream rest frame quantities respectively. The relative Lorentz factor between upstream and downstream is $\Gamma_{\mathrm{r}}=\Gamma_{\mathrm{sh}} / \sqrt{2}$. The mean energy gain is obtained by averaging over the cosine $\mu_{\mathrm{u} \rightarrow \mathrm{d}}$ and $\mu_{\mathrm{d} \rightarrow \mathrm{u}}^{\prime}$ of the penetration angles of the particle from upstream to downstream and downstream to upstream with respect to the direction of the boost. In the most optimistic case a high relative energy gain $\Delta E / E \sim \Gamma_{\mathrm{sh}}^{2}$ can be achieved (Vietri 1995). However, this gain is restricted to the first cycle if the initial particle distribution is isotropic. The particle distribution after one cycle becomes highly anisotropic, beamed in a cone of size $1 / \Gamma_{\text {sh }}$ and due to particle kinematics, the average relative gain drops to $\sim 2$ for the next crossings (Gallant and Achterberg 1999). Particle deflection in the cone can either proceed through its motion in a uniform magnetic field in the absence of scattering waves or by scattering with resonant waves with $k r_{\mathrm{g}} \sim 1$. Resonant scattering occurs only if the amplitude of the magnetic perturbations is small enough (Achterberg et al. 2001). The shock particle distribution in the test-particle limit shows a universal energy spectrum $N(E) \propto E^{-2.2}$ whatever the deflection upstream if scattering is effective downstream. This result is consistent with the index of the relativistic electron distribution producing synchrotron radiation in GRB afterglow (Waxman 1997). Note that this result has been obtained for an isotropic turbulence downstream. A more general formulation in terms of shock speed gives an energy index (Keshet and Waxman 2005) of 


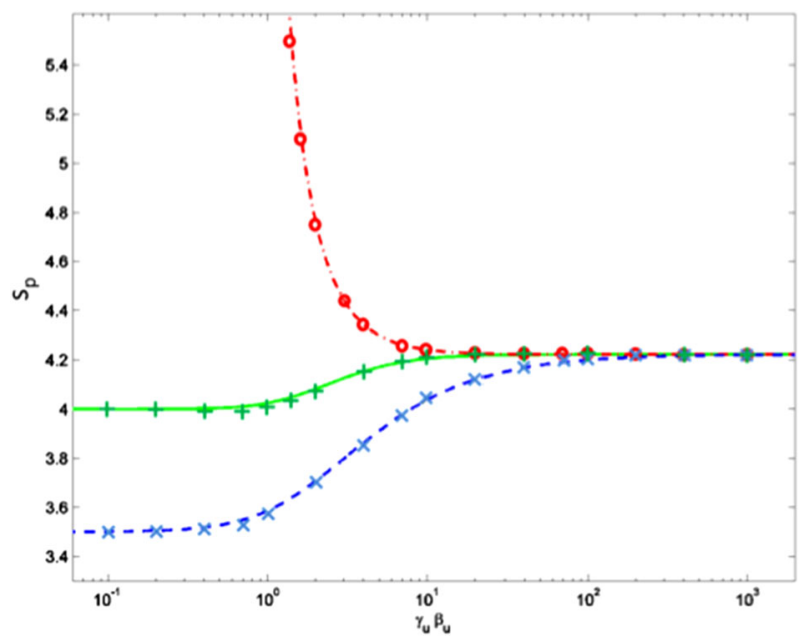

Fig. 5 Spectral index of the shock particle distribution as function of the shock Lorentz factor. Solid lines are the solutions obtained in Keshet and Waxman (2005) compared with the solutions of Kirk et al. (2000) plotted with symbols. A strong shock solutions with the Jüttner/Synge equation of state is shown in solid line and crosses, a strong shock with fixed adiabatic index $\gamma_{\mathrm{ad}}=4 / 3$ is shown in dashed line and $\mathrm{x}$-marks, and a shock with a relativistic gas where $\beta_{\mathrm{u}} \beta_{\mathrm{d}}=1 / 3$ is shown in dash-dotted line and circles. Image reproduced from Sironi et al. (2015). Copyright by Springer

$$
s=\frac{\beta_{\mathrm{u}}-2 \beta_{\mathrm{u}} \beta_{\mathrm{d}}^{2}+\beta_{\mathrm{d}}^{3}+2 \beta_{\mathrm{d}}}{\left(\beta_{\mathrm{u}}-\beta_{\mathrm{d}}\right)},
$$

where $\beta_{\mathrm{u} / \mathrm{d}}$ is the upstream/downstream fluid velocity normalized to c; $s=2+2 / 9 \simeq$ 2.2 is recovered in the ultra-relativistic limit $\left(\beta_{\mathrm{u}} \rightarrow 1\right.$ and $\left.\beta_{\mathrm{d}} \rightarrow 1 / 3\right)$. The value of the ultra-relativistic index has also been assessed by numerical simulations using a Monte-Carlo method (Bednarz and Ostrowski 1998; Achterberg et al. 2001) or a semi-analytical method based on the derivation of eigenfunctions in the particle pitchangle cosine of the solution of the diffusion-convection equation (Kirk et al. 2000). However, this spectrum is not properly universal in the sense that the index depends on the geometry of the turbulence (Lemoine and Revenu 2006).

Figure 5 shows the index of the shock downstream particle distribution as a function of the shock Lorentz factor from mildly relativistic to ultra-relativistic regimes for different equation of state of the relativistic gas (the quantity plotted is $s+2$ with our notation).

It appears that unless some particular turbulence develops around the shock front, Fermi acceleration associated with repeated shock crossings does not operate at relativistic shocks because of particle kinematic condition to cross the shock front (Begelman and Kirk 1990; Lemoine et al. 2006; Pelletier et al. 2009). This fact results from that relativistic shocks are generically perpendicular unless the magnetic field upstream is oriented within an angle $1 / \Gamma_{\text {sh }}$ along the shock normal. If the background turbulence around the shock is absent or if its coherence length is larger than the particle's gyroradius it can be shown that while returning to the shock from downstream to upstream the particle is unable to do more than one cycle and a half before 
being advected downstream (Lemoine et al. 2006). There are two necessary conditions for an efficient scattering and particle acceleration: (1) the turbulence which develops around the shock has to be strong, with a perturbed magnetic field such that $\delta B / B_{0}>1$, where $B_{0}$ is the background upstream magnetic field strength, (2) the perturbations have to be at scales smaller than the particle gyroradius (with respect to the total magnetic field) (Pelletier et al. 2009). One drawback of these conditions is that, as the micro-turbulence develops with a coherence scale smaller than $r_{\mathrm{g}}$, the spatial diffusion coefficient scales as $D \propto r_{\mathrm{g}}^{2}$, and as the acceleration timescale scales as $t_{\text {acc }} \propto D / U_{\mathrm{sh}}^{2} \propto E^{2}$ even when $U_{\mathrm{sh}} \rightarrow \mathrm{c}$, the time required to reach extremely high energies can become very large. This is the main reason which explains why ultrarelativistic GRB shocks $\Gamma_{\text {sh }} \gg 1$ cannot be at the origin of CRs with energies $\sim 100$ EeV (Lemoine and Pelletier 2010; Plotnikov et al. 2013; Reville and Bell 2014).

One note on the origin of the micro-turbulence We have seen that the onset of micro-turbulence is necessary for the Fermi process to operate at relativistic shocks. What type of micro-turbulence develops depending on shock velocity regimes and upstream medium properties?

The characteristic scale on which the micro-turbulence can develop is given by the CR precursor scale $\ell_{\text {prec }}$. It is either set by the regular gyration of particles reflected by the shock front, in which case $\ell_{\text {prec }}=r_{\mathrm{g}} / \Gamma_{\mathrm{sh}}^{2}$, or by diffusion, in which case $\ell_{\text {prec }}=r_{\mathrm{g}}^{2} / \ell_{\mathrm{c}}$, where $\ell_{\mathrm{c}}$ is the turbulence coherence scale. As the energy spectrum is softer than $E^{-2}$, low energy particles carry the bulk of free energy and generate most of magnetic perturbations. Hence, the scale over which micro-instabilities can develop in relativistic shocks is small. This restricts the number of instabilities to a few (Lemoine and Pelletier 2010). The nature of the dominant instability depends on two main parameters: the shock Lorentz factor $\Gamma_{\mathrm{sh}}$ (or momentum $\Gamma_{\mathrm{sh}} \beta_{\mathrm{sh}}$ for mildly relativistic shocks), and the upstream magnetization $\sigma_{\mathrm{u}}=B_{0}^{2} / 4 \pi\left(\Gamma_{\mathrm{sh}}\left(\Gamma_{\mathrm{sh}}-1\right) \mathrm{n}^{?} \mathrm{mc}^{2}\right.$, where $\mathrm{n}^{\star}$ is the ambient (upstream) proper gas density composed of particles of mass $m$ (Sironi et al. 2015; Marcowith et al. 2016). In weakly magnetized shocks $\left(\sigma_{\mathrm{u}} \leq 10^{-3}\right)$ the dominant instability is the electromagnetic filamentation or Weibel instability (Weibel 1959). Filamentation/Weibel instabilities grow due to the presence of two counterstreaming population of particles and produce modes in the direction perpendicular to the streaming direction (Fried 1959; Bret 2009). Plotnikov et al. (2013) discuss also the case of the oblique two-stream instability which can have a competitive growth rate with respect to the filamentation instability. Finally, the Buneman instability has been discussed as a source of electron heating in relativistic shock precursors (Lemoine and Pelletier 2011). At higher magnetization $\left(0.1>\sigma_{\mathrm{u}}>10^{-3}\right)$ a current driven instability either in subluminal (Bell 2004, 2005; Reville et al. 2006; Milosavljević and Nakar 2006) or superluminal shocks (Pelletier et al. 2009; Casse et al. 2013; Lemoine et al. 2014) can develop in the precursor. If $\sigma_{\mathrm{u}}>0.1$ then the gyration of particles in the background magnetic field gains in coherence and the shock is mediated by the synchrotron maser instability. This instability produces a train of semi-coherent large amplitude electromagnetic waves that escapes into the upstream medium (Gallant et al. 1992; Hoshino et al. 1992; Plotnikov and Sironi 2019). The interaction of this wave with the background plasma is a source of an efficient electron 
pre-heating up to equipartition with protons (Lyubarsky 2006; Sironi and Spitkovsky 2011). The question of whether this wave can lead to a significant particle acceleration is debated (e.g., Hoshino 2008; Iwamoto et al. 2017; Lyubarsky 2018).

\subsubsection{Progress with fully kinetic simulations}

Until late 2000s, most progress on the understanding particle acceleration at relativistic shocks were supported by Monte-Carlo simulations (e.g., Bednarz and Ostrowski 1998; Lemoine and Pelletier 2003; Ellison and Double 2004; Lemoine and Revenu 2006) or semi-analytic approaches (Kirk et al. 2000; Achterberg et al. 2001; Keshet and Waxman 2005). As pointed out by Bykov and Treumann (2011), recent advances were possible by employing fully kinetic PIC simulations. Self-consistent build-up of Fermi process was observed and a survey in which parameter space it operates was done (Spitkovsky 2008b; Martins et al. 2009; Sironi and Spitkovsky 2009, 2011; Sironi et al. 2013; Plotnikov et al. 2018). For instance, these simulations demonstrate that the Weibel-filamentation instability dominates in controlling the shock structure in weakly magnetized shocks, as predicted by Medvedev and Loeb (1999) and Gruzinov and Waxman (1999). Particle acceleration is correlated to the efficiency of triggering this instability. Typically, the non-thermal particles contain about $1 \%$ of total particle number and about $10 \%$ of total energy. The tail develops into a power-law with spectral slope $s \simeq 2.4$ that is close to the semi-analytic prediction of 2.2. The maximum energy of particles evolves in time as $E_{\max } \propto \sqrt{t}$ (Sironi et al. 2013) due to the smallscale nature of the magnetic turbulence (see above). In the small-angle scattering regime, the spatial diffusion coefficient of particles is $D \propto E^{2}$, unless the external magnetic field imposes a saturation that sets the maximal particle energy to be $E_{\max }>$ $e \delta B^{2} / B_{0} \ell_{\mathrm{c}}$ (Plotnikov et al. 2011; Marcowith et al. 2016). For typical parameters in ultra-relativistic shocks with $\Gamma_{\text {sh }} \sim 100$ propagating in the ISM with $B_{0} \sim 3 \mu \mathrm{G}$, this energy does not exceed $10^{16} \mathrm{eV}$. Section 4 presents more detailed discussions of these studies.

\subsubsection{Long term evolution}

The fate of the micro turbulence and, more generally, the long-term evolution of the weakly magnetized shocks remains the major unanswered question in relativistic (but also in non-relativistic) shock physics. As this micro turbulence is composed of initially short wavelength perturbations, these are expected to be rapidly damped by Landau damping downstream (Gruzinov 2001; Chang et al. 2008; Lemoine 2015). One possibility to overcome this effect would be to have some amounts of inverse cascade to generate large wavelengths or to rely one the perturbations generated upstream by high-energy particles and transmitted downstream. These possibilities, and others are detailed in Sect. 3.2 of Sironi et al. (2015).

\subsection{Reconnection in astrophysical flows}

Magnetic reconnection can occur in a collisional or in a collisionless plasma. The bulk part of the particles are accelerated to order of the Alvén speed and heated in the 
same process. A fraction of the particles can be accelerated to much higher velocities and form a power-law up to very high Lorentz factors. The power-law slopes can be harder than power-laws produced by a Fermi process, e.g. in collisionless shocks. In astrophysics, reconnection (REC) is a highly important process to accelerate particles.

In this introductory section, we briefly summarize some important concepts of magnetic reconnection. For any further details, we refer to recent reviews on the subject by Zweibel and Yamada (2009), Zharkova et al. (2011) (solar flares), Melzani (2014), Gonzalez and Parker (2016), Jafari and Vishniac (2018). A more deep insight into subjects directly related to this review, the acceleration of particles, will be given in Sect. 4.2.

Astrophysical objects where reconnection takes place Well-known REC sites in the solar systems are the upper chromosphere and the corona of the Sun as well as the magnetotail and the magnetopause of planets. There, predominately electrons are accelerated to very high speeds. REC may be partly responsible for heating the solar corona and thus for the existence of the solar wind. But highly accelerated particles are also a severe threat for spacecrafts and astronauts and even aircraft passengers. They are at the source of geo-magnetic storms which severely endanger communication and power grids on Earth. The demand for a better understanding of space-weather is one reason why in recent years the effort to understand REC has intensified and brought decisive new insights.

In outer space, REC was found to play a crucial role in the understanding of high-energy objects such as pulsars and their winds and nebulae, as well as magnetars, (micro-)quasars, and GRBs. In most of these objects, REC is partly a driver of their dynamics. Besides shocks and wave-turbulence, REC can accelerate particles to highest energies under such conditions. These particles and their interaction with the environment also inevitably contribute to the emission spectrum of such objects. In addition, they may be at the source of the production of high-energy neutrinos observed on Earth. REC in such objects is mostly relativistic in that the energy stored in the associated magnetic fields exceeds the rest mass energies of electrons and protons.

Figure 6 shows the parameter space of magnetic REC present in astrophysics and relates it to concrete systems. As can be taken from the figure, collisional and noncollisional REC equally contribute to the overall picture of magnetic REC in space. Indicated are as well different other regimes which will be discussed below. Fusion devices like ITER and TFTR and experimental setups like MRX, NGRX, MST, VTF are also shown.

\subsubsection{Collisional reconnection models}

Sweet-Parker model The first theory of magnetic reconnection was presented by (Sweet 1958) for a collisional plasma with resistivity $\eta ; \mathbf{J}=\eta \mathbf{E}$. Parker (1957) worked out the scaling relations presented below. It was soon clear that it is not always applicable, as this model predicts too slow events as compared to observations. The question how to make REC fast is still today in the center of the discussion and not generally solved. 

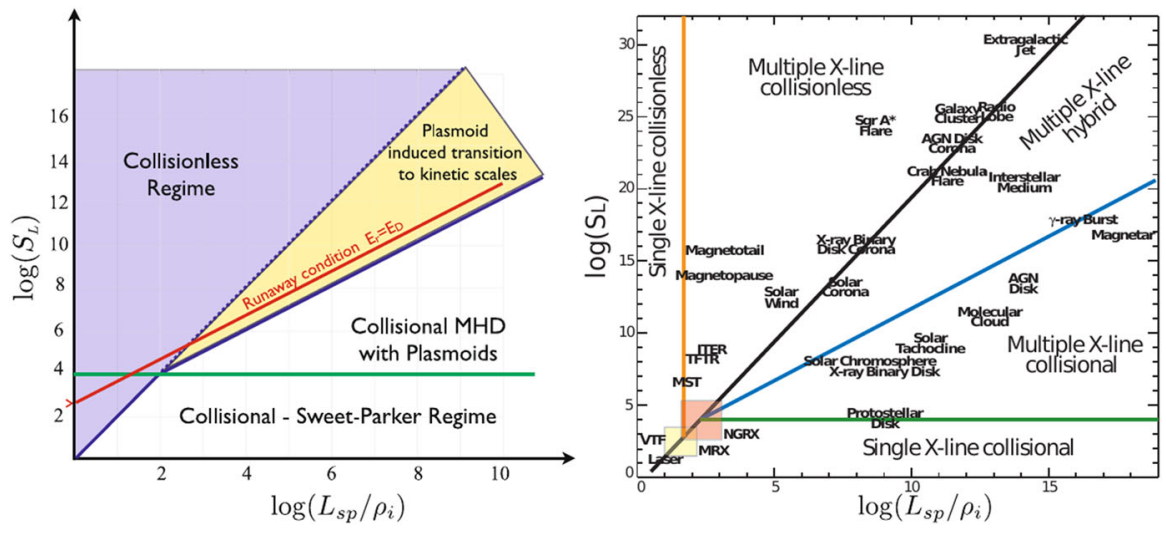

Fig. 6 Different REC regimes, as derived by Daughton and Roytershteyn (2012) (left panel) and Ji and Daughton (2011) (right panel). $S_{\mathrm{L}}$ is the Lundquist number defined by Eq. (17), $L_{\mathrm{sp}}$ the macroscopic system size, $\rho_{\mathrm{i}}$ the ion Larmor radius in the asymptotic magnetic field (including the guide field), not to be confused with the ion mass density. The red curve is computed for $\beta_{\mathrm{p}}=0.2$ and a REC rate of $R=0.05$. Images reproduced with permission from Daughton and Roytershteyn (2012), copyright by Springer; and from Ji and Daughton (2011). Copyright by AIP

Assume a collisional plasma with a certain resistivity. Then the induction equation and Ohm's law become, with $\mathbf{U}$ the flow velocity,

$$
\frac{\partial \mathbf{B}}{\partial t}=\nabla \wedge(\mathbf{U} \wedge \mathbf{B})+\eta \nabla^{2} \mathbf{B} ; \quad \mathbf{E}+\frac{\mathbf{U}}{c} \wedge \mathbf{B}=\frac{\mathbf{J}}{\sigma} .
$$

The non-ideal terms are the resistive diffusivity $\eta=c^{2} / 4 \pi \sigma$ in the induction equation and the resistive current in Ohm's law expressed in terms of the electrical conductivity $\sigma$. The non-ideal induction equation makes clear that there is a competition between the diffusion of the magnetic field (governed by the resistive time-scale $\tau_{R}$ ) and the ideal evolution (governed by the Alfvénic time scale $\tau_{\mathrm{A}}$ ). This balance is expressed by the magnetic Reynold's number, $R_{\mathrm{m}} \equiv \ell \cdot \tilde{U} / \eta$ with $\ell$ a characteristic length scale and $\tilde{U}$ a typical velocity of the system. In ideal MHD $R_{\mathrm{m}}>>1$ and REC is suppressed; whenever $R_{\mathrm{m}}<<1$ field diffusivity wins and REC becomes possible though not mandatory.

Figure 7 describes a 2D steady situation. Plasma from an outer ideal region flows in parallel to the $\mathrm{x}$-direction towards a dissipation region, which has a length-scale, $L$, and a thickness, $\delta$. The inflow velocity is just given by the $E \times B$-drift in the plasma. In the outer ideal region $\left(R_{\mathrm{m}}>>1\right)$, the plasma is frozen to the magnetic flux. This is no longer true in the diffusion region where the resistivity is dominant: the plasma decouples from the magnetic field. This opens the possibility that the field reconnects and plasma is expelled in the z-direction. These outflows are called exhausts.

Applying mass and energy conservation, non-compressibility, and that the field energy is dominant at the inflow and the kinetic energy of the particles at the outflow, two important relations follow: 


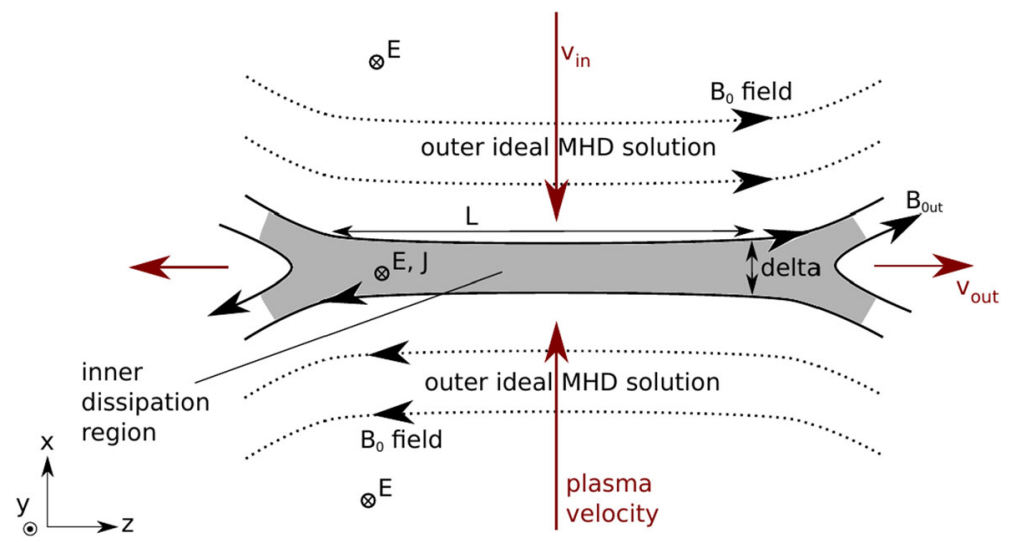

Fig. 7 Sweet-Parker model of magnetic reconnection. See details in the text. Image adapted from Melzani (2014)

$$
\begin{aligned}
U_{\text {out }} & =\sqrt{2} \frac{B_{0}}{\sqrt{4 \pi m n_{\text {in }}}}=\sqrt{2} U_{\mathrm{A}, \text { in }}, \\
\frac{\delta}{L} & =\frac{U_{\text {in }}}{U_{\mathrm{A}, \text { in }}}=M_{\mathrm{a}, \text { in }},
\end{aligned}
$$

where $U_{\mathrm{A} \text {,in }}$ is the Alfén speed and $M_{\mathrm{a} \text {,in }}$ the Alfénic Mach-number of the inflow. Thus, the outflow speed in the exhausts is of order of the Alfénic speed of the inflow. Assuming non-forced REC, the inflow is just $E \times B$-drift, $U_{\text {in }}=c E_{\mathrm{y}} / B_{0}=\eta / \delta$ and thus generically very sub-Alfénic. The Lundquist-number, $S_{\mathrm{L}}$, and the REC rate $R$ are defined as

$$
\begin{gathered}
S_{L} \equiv \frac{L U_{\mathrm{A}, \text { in }}}{\eta} \sim\left(\frac{L}{\delta}\right)^{2} \sim\left(\frac{U_{\mathrm{A}, \text { in }}}{U_{\text {in }}}\right)^{2} \sim\left(M_{\mathrm{a}, \text { in }}\right)^{2}, \\
R \equiv \frac{U_{\text {in }}}{U_{\text {out }}} \sim \frac{\delta}{L} \sim 1 / S_{L}^{1 / 2} .
\end{gathered}
$$

The Lundquist-number is equal to the magnetic Reynolds-number, $R_{\mathrm{m}}$, for the case where the typical velocity is equal the Alfvénic speed of the inflow. Highly conducting plasmas as found in astrophysics have high Lundquist numbers: laboratory plasma experiments typically have Lundquist numbers between $10^{2}$ and $10^{8}$. In astrophysics, they are higher, up to $10^{20}$ (Fig. 6, right panel) and thus Sweet-Parker reconnection rates very low-in contrast with what is observed.

The ratio between the incoming and outgoing energy flux in Sweet-Parker reconnection is $\propto 1 / S_{L}$. So, energy is indeed transferred from the magnetic field (incoming flux) to particles (outgoing flux). In the diffusive region with de-magnetized particles, the electric field can accelerate them. But there are other, most probably even more important acceleration mechanisms as will be discussed in Sect. 4.2.

REC rates of such high Lundquist numbers are much too low as compared to what is observed. This result can be translated to time-scales. The magnetic Reynolds-number can also be expressed as $R_{\mathrm{m}}=\tau_{\mathrm{R}} / \tau_{\mathrm{A}}$, where $\tau_{\mathrm{R}}$ is the resistive diffusion time-scale and 


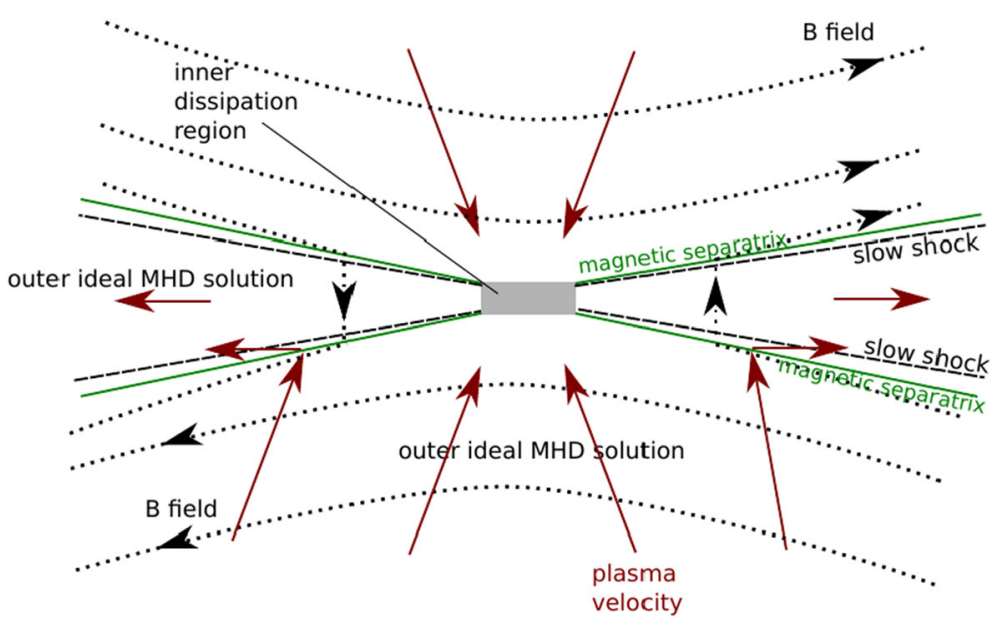

Fig. 8 Illustration of the fast REC Petschek model. Image adapted from Melzani (2014)

$\tau_{\mathrm{A}}$ Alfvén time-scale. In astrophysical environments with high Lundquist numbers it is thus found that $\tau_{\mathrm{R}}<<\tau_{\mathrm{A}}$. The typical Sweet-Parker REC time-scale is $\sim \tau_{\mathrm{A}}^{1 / 2} \tau_{\mathrm{R}}^{1 / 2}$. This indicates that Sweet-Parker REC is indeed faster than resistive diffusion of the magnetic field (scaling with $\tau_{\mathrm{R}}$ ). However, it is much too slow when compared to REC observed in astrophysical plasmas which scales as 10-100 $\tau_{\mathrm{A}}$. For instance, in flares of the solar corona, $S_{\mathrm{L}} \sim 10^{8}$ (see Fig. 6), $U_{\mathrm{A}} \sim 100 \mathrm{~km} \mathrm{~s}^{-1}$, and $L \sim 10^{4} \mathrm{~km}$. Thus, the Sweet-Parker-timescale is a few tens of days. Observed is a magnetic energy release within a few minutes to an hour. This major discrepancy is known as the fastness problem of Sweet-Parker REC.

On the other hand, numerical simulations based on resistive MHD as well as experiments such as the MRX, the Magnetic Reconnection Experiment (Ji et al. 1998) are in good agreement with the Sweet-Parker model. Clearly, the Sweet-Parker model has its deficits, in that it neglects dimensionality and any time-dependence, as well as viscosity, compressibility, downstream pressure, and, in particular, turbulence and is strictly valid only for a collisional plasma. There have been numerous papers addressing the fastness problem. Only in recent years, significant progress in this question has been made, see below.

Petschek model Petschek (1964) proposed a REC model in which the reconnection rate is nearly independent of the Lundquist number, $v_{\text {in }} / v_{\mathrm{A} \text {, in }} \approx \pi / 8 \ln S_{L}$ and thus REC is fast. The trick is to add, in close neighborhood to the separatrices slow shocks (left panel of Fig. 8) into the configuration. In this way, particles can be accelerated without having to pass through the inner dissipation region with resistive dissipation. Instead, magnetic energy can be conversed to kinetic particle energy in the shocks.

However, all resistive MHD simulation are in agreement with the Sweet-Parkermodel unless a localized anomalously large resistivity is used, mimicking that the mean free particle path becomes larger than the reconnection layer. Otherwise, shocks 


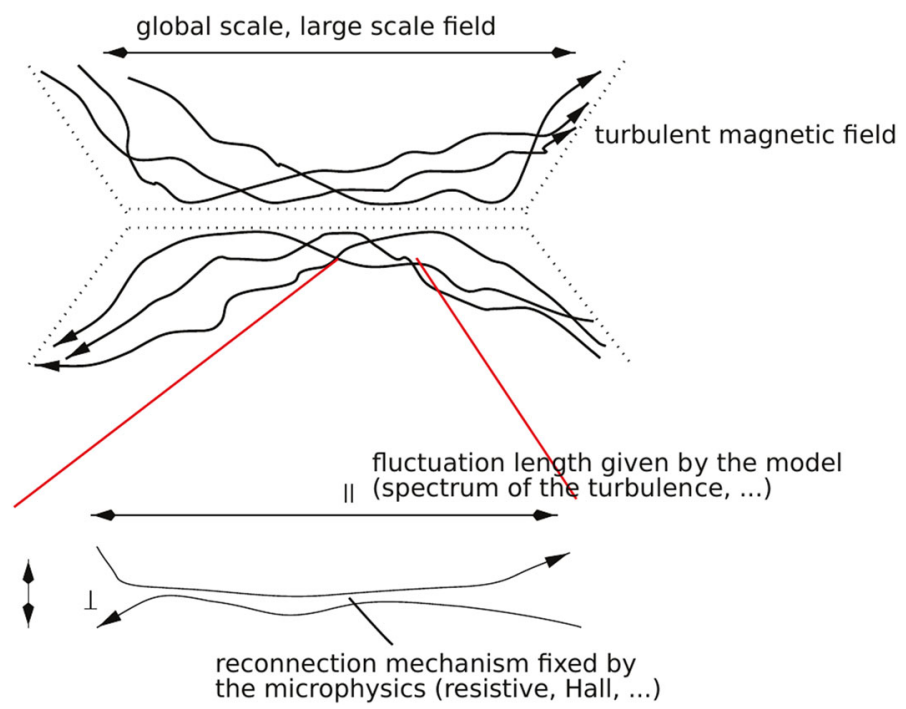

Fig. 9 Illustration of the fast REC stochastic model by Lazarian and Vishniac (1999). Image adapted from Melzani (2014)

are not observed in MHD simulations. Therefore, the Petschek-model is likely not a model of resistive MHD - though this is still a controversial question. However, recent PIC simulations in a collisionless plasma show an X-point and separatrix-structure in reconnection, which resembles somehow the Petschek-model (Higashimori and Hoshino 2012; Liu et al. 2012; Lapenta et al. 2015) —at least at scales larger than kinetic scales. There is also observational evidence that in the reconnection region of Earth's magnetotail, slow shocks are present (Eriksson et al. 2004). This discussion will be resumed in Sect. 4.2.

Turbulence External or self-generated in the REC process-seems to be the key process which allows resistive MHD REC to be fast. As indicated in Fig. 9 turbulent fluctuations allow to form many, much smaller scaled, reconnection spots along the global length, L, of the sheet. As shown in Lazarian and Vishniac (1999), the REC becomes thus much faster and is independent of the exact REC mechanism at each of these spots (Sweet-Parker, collisionless, ...). The exact result depends, however, on the nature of the turbulence and its fluctuation. Numerical simulations show good agreement with the analytic result (Kowal et al. 2009, 2012).

Simulations show that a Sweet-Parker like current sheet generates islands above a critical Lundquist number $S_{c} \sim 10^{4}$ (Daughton and Roytershteyn 2012). In relativistic flows, this critical number may be higher, $S_{c} \sim 10^{8}$ (Zanotti and Dumbser 2011). This is confirmed by newer investigations and linked to an extremely fast growing tearing instability of the current sheet (Del Zanna et al. 2016; Papini et al. 2018). This limit is indicated as the green line in the left panel of Fig. 6. 
Lapenta (2008) showed that a Sweet-Parker sheet setup in a Harris or force-free equilibrium sheet develops slow REC. On a much longer time-scale, tearing modes start to fragment the sheet and several X-points form. The exhausts of these X-points generate turbulence leading to multiple short lived REC regions, popping up randomly, frequently and at multiple locations simultaneously. Consequently, fast REC sets in. Similar findings for 3D resistive reconnection are presented by Oishi et al. (2015). By linking such self-generated turbulence with external turbulence, Lapenta and Lazarian (2012) formulate a united approach. So one may, with still some care, conclude that also collisional, resistive REC is fast, at least under certain conditions.

\subsubsection{Collisionless reconnection}

On length scales shorter than the ion inertial length $c / \omega_{\mathrm{p}, \mathrm{i}}$ where $\omega_{\mathrm{pi}} \equiv \sqrt{4 \pi n_{\mathrm{i}} Z^{2} e^{2} / m_{\mathrm{i}}}$ is the ion plasma frequency, ions decouple from electrons and the magnetic field becomes frozen into the electron fluid rather than the bulk plasma. Consequently, other terms than just resistivity start to contribute to the Ohm's-law. For instance, based on a two-fluid non-relativistic plasma model, Melzani (2014) derives a more complex Ohm's-law for electrons:

$$
\begin{aligned}
\underbrace{}_{\text {E-field in the ion plasma frame }} & \underbrace{\mathbf{E}+\frac{\mathbf{v}_{\mathrm{i}}}{c} \wedge \mathbf{B}}_{\text {Hall term }}=\underbrace{\frac{1}{n_{\mathrm{e}} \mathrm{e}} \wedge \mathbf{J}}_{\text {electron bulk inertia }}-\underbrace{\frac{m_{e}}{\mathrm{e}}\left(\frac{\partial \mathbf{v}_{\mathrm{e}}}{\partial t}+\mathbf{v}_{\mathrm{e}} \cdot \nabla \mathbf{v}_{\mathrm{e}}\right)}_{\mathrm{e}^{-}}-\underbrace{\frac{1}{n_{\mathrm{e}} \mathrm{e}} \nabla \cdot P_{\mathrm{e}}}_{\mathrm{e}-\mathrm{i} \text { collisions inertia }} \\
& +\underbrace{\frac{\chi}{\left(n_{\mathrm{e}} \mathrm{e}\right)^{2}}}_{\mathrm{e}-\mathrm{e} \text { collisions }} \mathbf{J}
\end{aligned}
$$

Here, $n_{e}$ is the electron number density, $v_{i}, v_{e}$ the ion and the electron velocity respectively, c the speed of light, $e$ the elementary charge, $\chi$ accounts for the effect of collisions between electrons and ions which, in general can be anisotropic and depend on the magnetic field orientation. $\chi_{e} \nabla^{2} \mathbf{v}_{\mathrm{e}}$ describes the electron viscosity due to electron-electron collisions. $P_{\mathrm{e}}$ is the pressure tensor

$$
P_{\mathrm{e}}=\int \mathrm{d}^{3} \mathbf{v} m_{\mathrm{e}}\left(v_{\mathrm{a}}-\bar{v}_{\mathrm{a}}\right)\left(v_{\mathrm{b}}-\bar{v}_{\mathrm{b}}\right)
$$

with $a, b=x, y, z$, and $\bar{v}$ is the mean velocity. Electron inertia, both thermal and bulk, now contribute to the non-ideal terms. In particular, if the plasma is completely collisionless, $\left(\chi=\chi_{e}=0\right)$, these are the only contribution of non-idealness of the plasma.

The sketch in the left panel of Fig. 10 shows that the dissipation region now is subdivided into a larger ion dissipation region with a size of $\delta_{\mathrm{i}}$ and a smaller electron dissipation region, sized to $\delta_{\mathrm{e}}$. Here, $\delta_{\mathrm{i}, \mathrm{e}}$ denotes the ion and electron inertial length.

On these scales, the Hall effect becomes important, because now the magnetic field lines are advected with the electrons while the ions no longer follow this motion. The Hall term is not responsible for REC as it appears when the magnetic flux is 

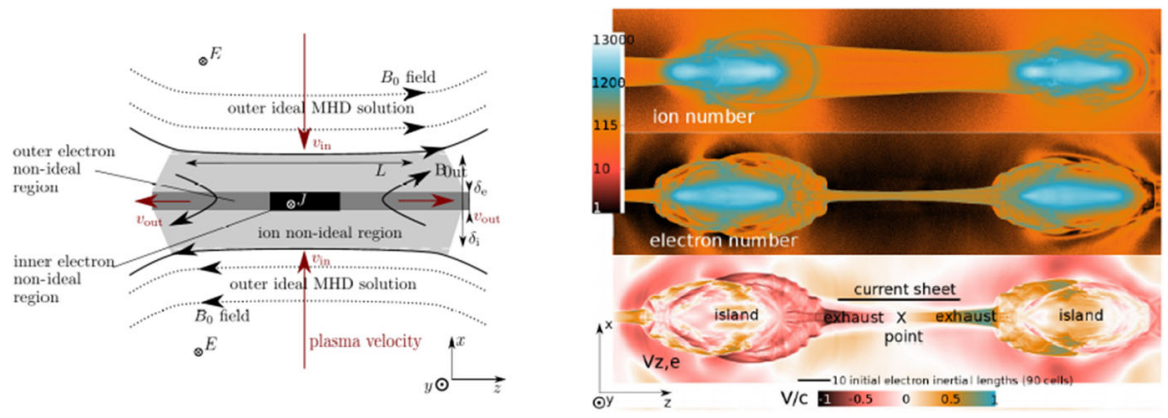

Fig. 10 Collisionless REC in a electron-ion plasma. Left panel: sketch of REC on a scale smaller than the ion inertial length. The diffusion regions for ions are much larger than that for electrons. The current sheet is more like an X-point than a double y-point. Ion trajectories normally do not pass through the electron non-ideal region. Image adapted from Melzani (2014). Right panel: X-point, exhausts and islands from a collisionless electron-ion PIC-simulation of REC using a mass-ration $m_{\mathrm{i}} / m_{\mathrm{e}}=25$. Visibly, the ion diffusion regions is about a factor of $5\left(\delta_{\mathrm{k}} \sim \sqrt{m_{\mathrm{k}}}, k=e, i\right)$ larger than the electron diffusion region. Image adapted from Melzani et al. (2014a)

still frozen to the motion of electrons. However, there is a debate whether it may contribute to the fastness of REC as it allows to accelerate electrons to higher speeds, increasing the bulk inertia. As can be taken from the right panel of Fig. 10 this two-layer picture derived from a two-fluid model is quite accurately reproduced by full kinetic simulations though the two-fluid model will not provide the full picture as effects like wave turbulence, Landau-damping and particle acceleration to speeds much higher than the Alfvén speed. Fully collisionless REC is found to be always fast. It will be further addressed in Sect. 4.2.

\subsubsection{Other effects}

Dimensionality REC in 3D shows a variety of new features. In some cases still a separatrix-like reconnection as in 2D can be observed, but there are also many other cases. It is not the place to discuss this here in detail. A good summary can be found in Melzani (2014).

Fat tails and high-energy power-laws Magnetic reconnection is efficient to accelerate particles, both in the collisional and collisionless regime. The typical speed of accelerated particles is the local Alfvén speed. If the flow is highly magnetized, this speed can be close to the speed of light. But kinetic simulations have revealed that the distribution function of accelerated particles have fat tails and power-laws up to very large relativistic Lorentz factors (Cerutti et al. 2013; Melzani et al. 2014b; Sironi and Spitkovsky 2014; Werner et al. 2018; Ball et al. 2018). Different acceleration mechanism are here at work which will discussed in Sect. 4.2.

Driven reconnection Reconnection sites are normally embedded in a large scale environment which is dynamic as well: jets, accretion disks, stellar winds, stellar atmospheres and coronae. Some of these environments are turbulent flows. As seen above, 
this can decisively accelerate the REC process. But also directed large scale flows-as compared to the diffusion region or X-point where REC actually happens-can significantly accelerate REC in that they provide significantly higher inflow velocities. Therefore, much more magnetic flux can be carried from larger scales to the reconnection site. The timescale of the forcing also proves to be important (Pei et al. 2001; Pritchett 2005; Ohtani and Horiuchi 2009; Klimas et al. 2010; Usami et al. 2014, 2018).

Multi-scale and multi-physics problem As was seen so far, REC is a multi-scale problem. Large scale MHD flows can have a significant impact on the rate and the energetics of REC. Another scale is the transition to a diffusive regime which "prepares' for REC, e.g., a Sweet-Parker reconnection sheet. Such sheets may break apart, introducing even smaller scales. This cascade in scales likely ends on kinetic scales. There also the physics may change, from a collisonal to a collisionless regime. Another important point is the scale-difference in mass between electrons and ions, which also translates into differently scaled diffusive regions, the ion-diffusion region being about $42.85\left(\equiv \sqrt{m_{p} / m_{e}}\right)$ times bigger than the electron diffusion region. And the different spatial lengths translate into equally different temporal scales. Magnetization and with it the ratio between an inertial length and the gyroradius yet complicates the situation.

But one has to address also other physical processes which influences the REC process. Outstanding here are radiative processes like synchrotron emission which directly changes the gyroradius. In an environment which is rich of photons, Compton scattering and Bremsstrahlung become important. More and more such processes are being addressed (Kirk and Skjæraasen 2003; Jaroschek and Hoshino 2009; Cerutti et al. 2013; Beloborodov 2017; Uzdensky 2016; Werner et al. 2019).

Multi-scale, multi-physics simulations demand for special techniques which are now in the course of being developed (Tóth et al. 2005; Daldorff et al. 2014; Tóth et al. 2012; Innocenti et al. 2013; Markidis et al. 2014; Ashour-Abdalla et al. 2015; Rieke et al. 2015; Lapenta et al. 2016; Tóth et al. 2016; Makwana et al. 2017; Lapenta et al. 2017; Lautenbach and Grauer 2018; Gonzalez-Herrero et al. 2018; Usami et al. 2018). We will come back to the issue in Sect. 4.2.

\subsection{Laser plasma experiments}

Over the past four decades, tremendous progress in the development of high-energy and high-power laser systems has brought the scientific community with the possibility to reproduce, in the laboratory, various scenarios relevant to astrophysics, space physics and planetology. This opened a new avenue for the development of so-called Laboratory Astrophysics, a field of growing activity that federates several communities [among which but not restricted to astrophysicists and (laser-)plasma physicists] and relies on the joint development of novel experimental and numerical capabilities.

In this section, we briefly review some key experiments focusing on the study of collisionless shocks and magnetic reconnection in laser-created plasmas ${ }^{7}$ The reader,

\footnotetext{
7 Other experimental facilities such as the Z-pinch machines (Remington et al. 2006) or the Large Plasma Device (LAPD) at UCLA (CA, USA) (Gekelman et al. 1991, 2016) also offer interesting opportunities for laboratory astrophysics, but are not discussed in this review.
} 
interested in other branch of laboratory astrophysics using laser-plasma experiments, will find interesting material covered in the review articles by Ripin et al. (1990), Rose (1994), Takabe et al. (1999), Remington et al. (1999), Remington et al. (2006), Takabe et al. (2008). These reviews cover topics ranging from warm dense matter, to equation of states and their application to planetology, opacities relevant to stellar interiors, or experiments investigating the hydrodynamics and magnetohydrodynamics of supernovae and (collisional) shocks.

In addition to presenting some of the main experimental results on collisionless shocks and magnetic reconnection, this section also aims at providing the reader with the characteristic parameters and conditions that can be created in the laboratory. To do so, we first introduce, in Sect. 2.6.1, the two main classes of lasers used for laboratory astrophysics. Then, in Sect. 2.6.2 we discuss the conditions that have to be met to ensure that collisional effects can be neglected. Finally, Sects. 2.6.3 and 2.6.4 summarize some of the key experimental results obtained on collisionless shock formation and magnetic reconnection.

\subsubsection{Overview of laser facilities and characteristic parameters}

Two main classes of laser systems are today used to support laboratory astrophysics research. First, high-energy density laser facilities delivering long (nanosecond) energetic (from few kJ up to 10 s of $\mathrm{kJ}$ ) light pulses have already allowed to reproduce various astrophysics-relevant scenarios, from warm dense matter studies, to the physics of hydrodynamic (radiative or not) shocks (Remington et al. 2006). Second, ultra-high intensity laser facilities deliver short (from few tens of femtoseconds to few picoseconds) light pulses that once focused onto a target allow to reach very high intensities. Even though laboratory astrophysics studies on this second class of laser systems is still in its infancy, recent developments of petawatt (and multi-petawatt) laser systems worldwide open new possibilities.

In this section, we report on some of the prominent laser facilities that are currently operating or will soon operate. Figure 11 lists these facilities as a function of the delivered energy and peak-power (the corresponding pulse durations are also indicated).

High-energy density lasers The development of high-energy density (HED) laser systems delivering energies of few tens of kilo-Joule (kJ) up to the Mega-Joule (MJ) over few to tens of nanoseconds (ns) has been strongly pushed forward by inertial confinement fusion programs (Atzeni and Meyer-Ter-Vehn 2004). Most of the experimental work that will be discussed in what follows has been performed on such laser systems. Various HED laser systems are today available, most of which are multi-beam facilities. Each beam can deliver ns pulses with few to $10 \mathrm{~kJ}$ (i.e., hundreds of beams are used on MJ-class laser systems) that, once focused onto target, allow to reach moderately high intensities of $10^{13}$ to a few $10^{15} \mathrm{~W} / \mathrm{cm}^{2}$. HED laser technology is based mainly on $\mathrm{Nd}$ :Glass amplifiers, which provide light beams at a (central) wavelength of $\sim 1.05 \mu \mathrm{m}$, but often use frequency doubling of tripling techniques, so that the operating wavelength can be decreased to $\sim 0.53 \mu \mathrm{m}$ (doubling) or $\sim 0.35 \mu \mathrm{m}$ (tripling). 


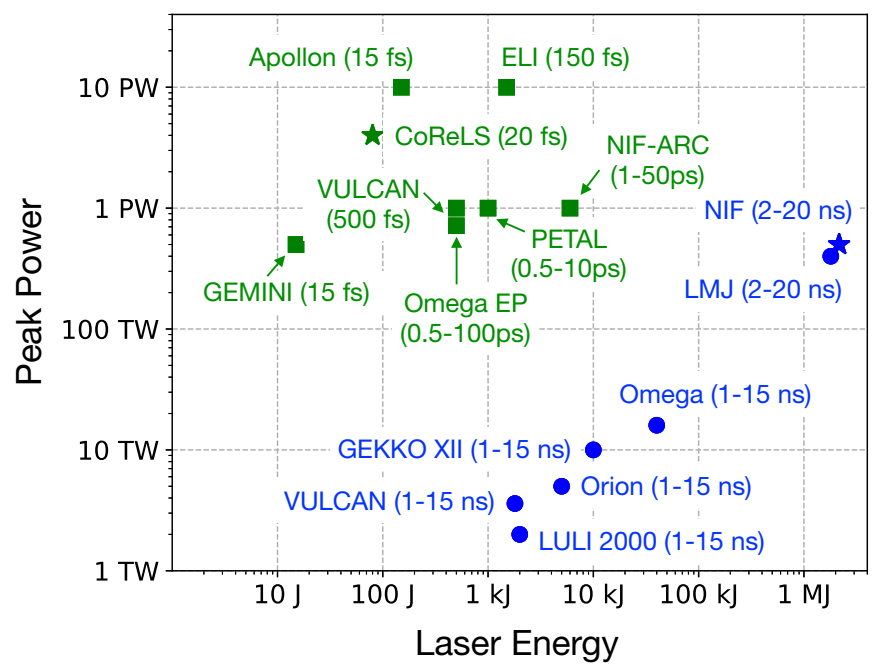

Fig. 11 Prominent laser facilities presented as a function of the delivered laser energy and peak power. High-energy density (HED) laser facilities are shown in blue, ultra-high-intensity (UHI) laser facilities in green. The laser characteristics (energy, power and typical pulse duration) are indicative

Among the prominent facilities are-at the multi-kJ level-the LULI $2000 \operatorname{laser}^{8}$ in France, the Orion ${ }^{9}$ and VULCAN ${ }^{10}$ facilities in the UK, the GEKKO XII facility ${ }^{11}$ in Japan, and the Omega laser ${ }^{12}$ in Rochester, US. Two mega-joule-class lasers are also operating or under construction: the National Ignition Facility (NIF) ${ }^{13}$ in Livermore, California, started operating in the early 2010s. The Laser-MegaJoule (LMJ) ${ }^{14}$ is still under construction in the South-West of France. Note that the MJ-energy level is achieved by combining hundreds of $10 \mathrm{~kJ}$ nanosecond laser beams.

Ultra-high intensity lasers High-power ultra-high intensity (UHI) laser facilities provide light pulses of moderate energy (from few tens of Joule to few kilo-Joule) but of a very short duration (from tens of femtoseconds to a few picosecondes) that, when focused onto a target, allow to reach tremendous intensities (beyond $10^{18} \mathrm{~W} / \mathrm{cm}^{2}$ ). At such intensities, electrons rapidly_in less than an optical cycle-become relativistic, and such UHI laser can help drive extremely fast, potentially relativistic, flows of plasmas.

Among the UHI lasers that are today considered for laboratory astrophysics studies, many are coupled to HED facilities. This is the case for instance of the PETAL ${ }^{15}$ and

\footnotetext{
8 https://portail.polytechnique.edu/luli/fr/installations/luli2000 (in French).

9 https://www.awe.co.uk/what-we-do/science-engineering-technology/orion-laser-facility/.

10 https://www.clf.stfc.ac.uk/Pages/Vulcan-laser.aspx.

11 http://www.ile.osaka-u.ac.jp/eng/facilities/gxii/index.html.

12 http://www.lle.rochester.edu/omega_facility/omega/.

13 https://lasers.llnl.gov/.

14 http://www-lmj.cea.fr/.

15 http://www.enseignementsup-recherche.gouv.fr/cid99515/petawatt-aquitaine-laser-petal.html (in French).
} 
NIF-ARC ${ }^{16}$ petawatt-class lasers coupled to the LMJ and NIF facilities, respectively, which deliver petawatt-level light pulses with energy of a few $\mathrm{kJ}$ and duration in the picosecond range. LULI 2000, VULCAN and ORION also have short (picosecond) pulse beamlines that deliver energy up to a few 100s J.

Other UHI facilities are also available that are not coupled to HED laser systems. This is the case e.g. of the femtosecond laser GEMINI ${ }^{17}$ in UK. Delivering $15 \mathrm{~J}$ in $30 \mathrm{fs}$, GEMINI has been used e.g. to produce dense electron-positron clouds which were used to drive current instabilities in a Helium plasma (Warwick et al. 2017). Let us further note that the most powerful laser is today the CoReLS ${ }^{18}$ in Gwanju, South-Korea, that has recently delivered 4 PW pulse (Nam et al. 2018). In addition the Apollon ${ }^{19}$ laser (in construction on the Plateau de Saclay, $20 \mathrm{~km}$ south of Paris, France) and the ELI project ${ }^{20}$ aim at reaching the unprecedented power of $10 \mathrm{PW}$ within the next few years (in light pulses of a few tens to few 100s fs). Laboratory astrophysics studies are envisioned on these facilities.

\subsubsection{The collisionless regime}

As previously stated, this section focuses on collisionless laser-plasma experiments and on the physics of collisionless shocks and magnetic reconnection in particular. The first observations of a collisionless coupling in laser-created plasmas date back to the early 1970s (Cheung et al. 1973), and very early the question collisionality effects arose, see, e.g., the work by Dean et al. (1971) and following exchange (Wright 1972; Dean et al. 1972).

The first (theoretical) investigations of collisionless shock experiments actually addressed this issue (Drake and Gregori 2012; Park et al. 2012; Ryutov et al. 2012). These works proposed the first designs and scaling laws to reproduce electrostatic or Weibel-mediated shocks (see Sect. 4 for complementary definitions) in laser-plasma experiments, and addressed the potential effects of particle collisions (and how to mitigate them) in counter-streaming plasma flows.

Of particular importance are collisions in between counter-streaming ions of the two flows (inter-flow collisions) that can have a dramatic effect on the shock formation. Indeed, and as stressed by Drake and Gregori (2012), the mean-free-path for ion-ion collisions measures the length over which an ion (subject to multiple scatterings/collisions) sees its velocity deflected by $90^{\circ}$. Hence, collisional effects will effectively isotropize the flow over a characteristic given by this mean-free-path and collisional shocks are known to develop on this spatial scale. Conducting a collisionless shock experiment thus requires that this (inter-flow) collision greatly exceeds the characteristic length of shock formation. As will be detailed in the following Sect. 2.6.3, it turns out that this condition can be "easily" met in electrostatic and to some extent magnetized shock experiments. In the case of Weibel-mediated shocks, entering the

\footnotetext{
16 https://lasers.llnl.gov/science/photon-science/arc.

17 https://www.clf.stfc.ac.uk/Pages/Laser-system-Gemini.aspx.

18 https://www.ibs.re.kr/eng/sub02_03_05.do.

19 http://cilexsaclay.fr/Phocea/Vie_des_labos/Ast/ast_technique.php?id_ast=9 (in French).

20 https://eli-laser.eu/.
} 
collisionless regime requires extremely fast (several $1000 \mathrm{~s} \mathrm{~km} / \mathrm{s}$ ) flows overlapping for a sufficient time accessible only on the most energetic (MJ-class) laser systems (Park et al. 2012).

In addition to inter-particle collisions, internal collisions between ions of the same flow can also be of importance, in particular as the ion temperature in the flow is quite low (at least before shock formation). This issue is briefly addressed by Drake and Gregori (2012) and Ryutov et al. (2012). Yet their impact on the development of instabilities such as the ion Weibel instability, or shock formation remains unclear. While it is difficult to claim that these (internal) collisions may not strongly modify the physics of shock formation, this may be checked by careful numerical modeling, e.g. relying on kinetic (Particle-In-Cell) simulations including collisional effects (see Sect. 3.3.2).

Last, Drake and Gregori (2012) discussed the possible impact of electron-ion collision on the dissipation of the magnetic structures that play a central role in the formation of Weibel-mediated shocks; and on longer time on particle acceleration. The authors showed that such collisions may indeed impact the small scale magnetic structures, but will most likely no impact the larger scale structures that develop on the scale of the ion skin-depth and thus the formation of Weibel-mediated shocks.

\subsubsection{Collisionless shock experiments}

As previously mentioned, evidences of collisionless processes in the presence of counter-streaming laser-produced plasmas were reported is the early 1970s. The first reported observation of a collisionless shock in a laser-created plasma ${ }^{21}$ dates back to Bell et al. (1988). This experiment was conducted on the VULCAN laser (Ross et al. 1981) at the Rutherford Laboratory (UK) where two laser pulses, each delivering 120J over $18 \mathrm{~ns}$ (FWHM), were focused in a $50 \mu \mathrm{m}$-diameter spot onto a flat carbon target. The resulting laser-produced ablation plasma had a density $\sim 10^{18} \mathrm{~cm}^{-3}$ and velocity of a few 100s of km/s. It collided with an obstacle (located $250 \mu \mathrm{m}$ away from the ablated target). The experiment led to the formation of density structures that were interpreted as collisionless bow shocks. In this experiment, all mean-free-paths were larger than the $\mathrm{mm}$, while the width of the observed shock front ranged from 0.01 to $0.05 \mathrm{~mm}$. The nature of the shock — either electrostatic or weakly magnetized-was however not fully defined.

Electrostatic shocks Following this pioneering work, and since the late 2000s in particular, collisionless electrostatic shocks have been abundantly produced in laserplasma experiments. These later developments were accompanied by both strong developments in diagnostics, and the use of kinetic (Particle-In-Cell) simulations to support the experimental effort.

For instance, Romagnani et al. (2008) demonstrated the creation of an electrostatic shock following the sudden expansion of a plasma into a rarefied gas. In this experiment carried out on the LULI 100 TW laser facility, one laser pulse with duration 470 ps and energy of a few tens of J was focused onto a Tungsten or Aluminium foil. Quickly

\footnotetext{
${ }^{21}$ Collisionless shock waves were obtained in plasma experiments, albeit not using lasers, since the mid1960s [see Strokin (1985) and references therein]. Already these studies where motivated by space-plasmas and astrophysics.
} 

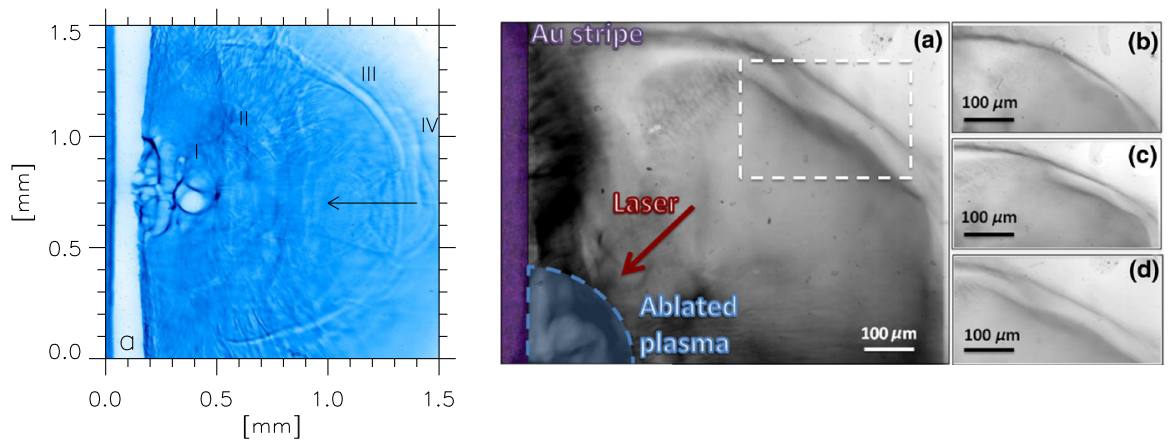

Fig. 12 Left: typical proton radiographies of laser-driven electrostatic shocks. Region I shows strong modulations associated with the ablating plasma, regions II and III show different structures that are interpreted as shock waves propagating ahead of the ablating plasma, while the modulated pattern in Region IV is located ahead of the shock front and possibly associated with a reflected ion bunch. The arrow indicates the laser beam direction. Right: shock structure 150 (b), 160 (c) and 170 ps (d) after the beginning of the interaction. The different times are accessible, for a single shot, by selecting protons with different energies [11.5 MeV for panel (b), $10 \mathrm{MeV}$ for panel (c) and $9 \mathrm{MeV}$ for panel (d)] as their time of flight from their source to the shock structure is different. Images reproduced with permission from (left) Romagnani et al. (2008) and (right) from Ahmed et al. (2013), copyright by APS

heated, the ablated foil expanded in the surrounding media and drove the formation of a collisionless electrostatic shock wave, about $1 \mathrm{~mm}$ away from the target, that was propagating at a velocity close to the ion acoustic velocity $\sim 200-400 \mathrm{~km} / \mathrm{s}$. The shock was diagnosed using proton radiography (Borghesi et al. 2001), a technique that is now central to the study of collisionless shock in laser-plasma experiments. It relies on the deflection (in the electromagnetic fields developed at the shock front) of protons created by a second ultra-short $(\sim 300 \mathrm{fs})$ ultra-intense $\left(\gtrsim 10^{18} \mathrm{~W} / \mathrm{cm}^{2}\right)$ laser pulse. The proton radiography is recorded onto dosimetrically calibrated radiochromic films (RCFs), as shown in Fig. 12.

Other experiments (Kuramitsu et al. 2011; Ahmed et al. 2013; Morita et al. 2013) have similarly reported the formation of electrostatic collisionless shock waves using ablating plasmas, either in direct interaction with a standing (background) plasma, or in counter-streaming plasma flows.

Magnetized shocks The first observation of a magnetized collisionless shock motivated by astrophysics studies was claimed by Niemann et al. (2014) combining the use of a $25 \mathrm{~ns}-200 \mathrm{~J}$ laser and the LAPD. In this experiment, the LAPD was used to produce a large scale $(17 \mathrm{~m} \times 0.6 \mathrm{~m})$ low density $\left(10^{12}-10^{13} \mathrm{~cm}^{-3}\right)$ and temperature $\left(T_{\mathrm{i}}=1 \mathrm{eV}, T_{\mathrm{e}}=6 \mathrm{eV}\right)$ hydrogen plasma embedded in an external magnetic field $B_{0}=300 \mathrm{G}$. The $10^{13} \mathrm{~W} / \mathrm{cm}^{2}$ ns laser pulse was fired at a solid polyethylene target embedded inside the magnetized plasma, which launched a denser $\left(8 \times 10^{16} \mathrm{~cm}^{-3}\right)$ slightly warmer $\left(T_{\mathrm{e}} \sim 7.5 \mathrm{eV}\right)$ carbon ion plasma at a velocity of $\sim 500 \mathrm{~km} / \mathrm{s}$ directed perpendicular to the magnetic field. The interaction of this super-Alfvénic plasma with the ambient (LAPD) plasma led — through a collisionless coupling — to the formation of a magnetic piston and then to the formation of self-sustained magnetosonic shock, supported by the ambient ions and propagating away from the piston at a velocity of $\sim 370 \mathrm{~km} / \mathrm{s}$ (corresponding to an Alfvénic Mach number $M_{A} \sim 2$ ). The reported 


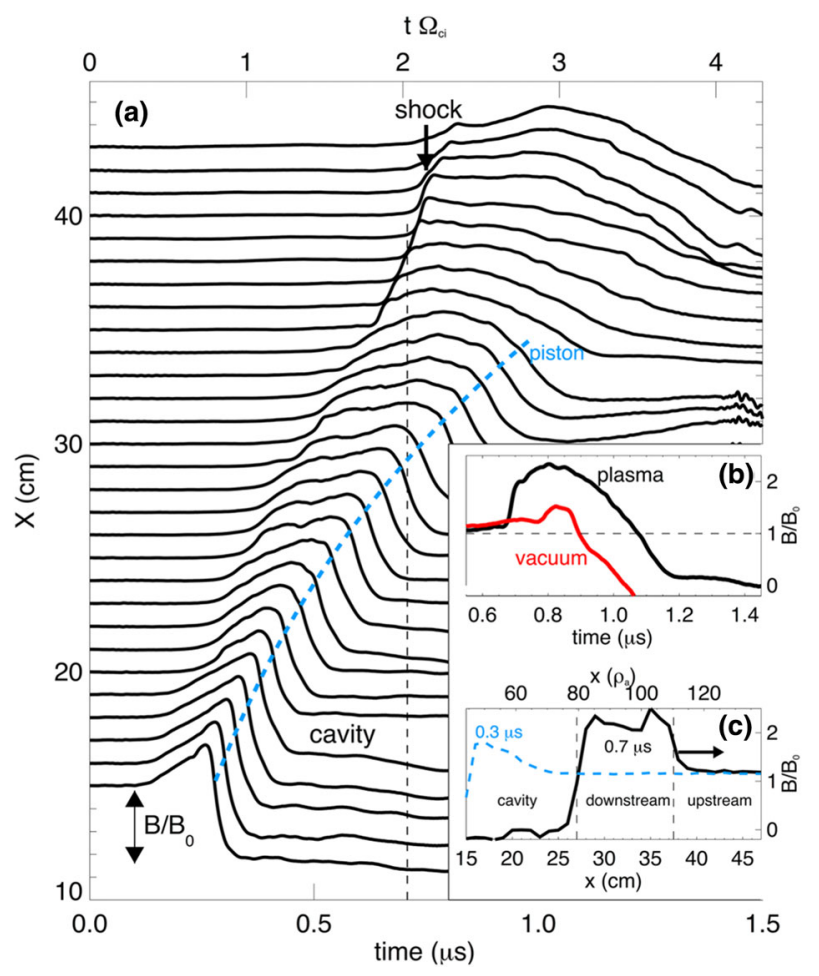

Fig. 13 Magnetized shock experiment at the Large Plasma Device (LAPD, University of California Los Angeles). a Magnetic $(B)$ field structure as a function of position ( $x$ is the direction of the carbon plasma flow, transverse to the direction of the background magnetic field) and time. b Temporal evolution of the magnetic field at $x=35 \mathrm{~cm}$ with (black) and without (red) the ambient plasma. c Spatial profile of the magnetic at two different times, $0.3 \mu \mathrm{s}$ (before shock formation, dashed light blue line) and $0.7 \mu \mathrm{s}$ (after shock formation, solid black line). Image reproduced with permission from Niemann et al. (2014), copyright by AGU

measurements (shock velocity and magnetic field compression $B / B_{0} \sim 2$ ) were found to be consistent with Rankine-Hugoniot conditions as well as with two-dimensional, collisionless, simulations performed using an electromagnetic Darwin code (Winske and Gary 2007). Note that, as illustrated in Fig. 13, for this particular experiment, the use of the LAPD allowed to follow the magnetic piston and shock formation over large spatial (few tens of $\mathrm{cm}$ ) and temporal (few microseconds) scales, well beyond what is usually accessible using HED or UHI laser systems.

The first laboratory observation of a laser-driven high-Mach-number magnetized collisionless shock was reported by Schaeffer et al. (2017a). This experiment was conducted on the Omega EP laser facility at Rochester (US) and built up on the concept of magnetic piston used in, e.g, the previous experiment by Niemann et al. (2014). However, it relied solely on the use of the HED laser Omega EP and allowed to create super-critical magnetized shocks with (magnetosonic) Mach number $M_{\mathrm{ms}} \equiv$ $u_{\mathrm{sh}} / c_{\mathrm{ms}} \sim 12$, with $u_{\mathrm{sh}} \sim 700 \mathrm{~km} / \mathrm{s}$ the measured shock velocity $\left(c_{\mathrm{ms}}^{2}=u_{A}^{2}+c_{s}^{2}\right.$, $u_{A}$ and $c_{S}$ being the (upstream) Alfvén and ion acoustic velocities, respectively). To 


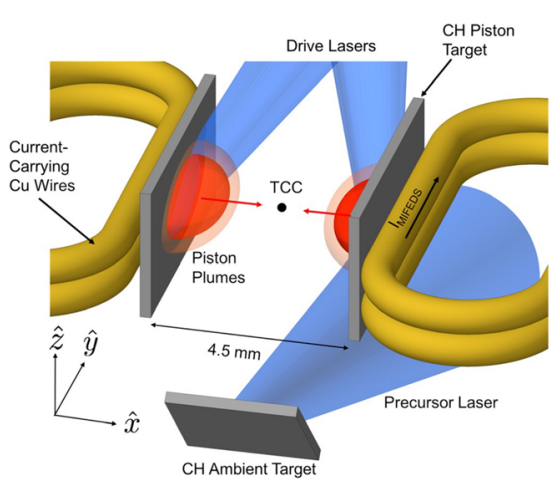

(a)

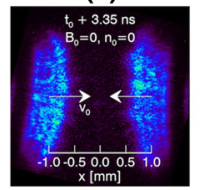

(d)

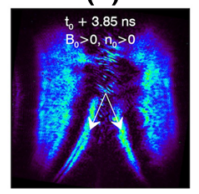

(b)

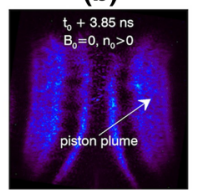

(e)

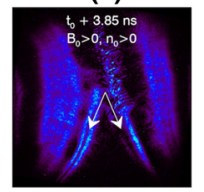

(c)

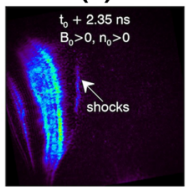

(f)

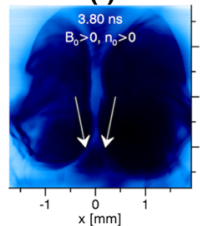

Fig. 14 Magnetized shock experiment at the Omega EP laser facility. (Left) simulation setup. (Right) panels (a-e) present the angular filter refractometry measurements for different configurations: a no background plasma, no external magnetic field (no shock is observed); b presence of a background plasma but no external magnetic field; $\mathbf{c}$ with both background plasma and external magnetic field but only one piston plume; $\mathbf{d}, \mathbf{e}$ in the presence of the two piston plumes and background plasma, with external magnetic fields in parallel and anti-parallel configuration, respectively. $\mathbf{f}$ Proton radiography signal revealing the strong magnetic field compression at the location of the shock structure. Images adapted from Schaeffer et al. $(2017 \mathrm{a}, \mathrm{b})$

do so, various beams of the Omega EP laser were used. A first beam, with energy $100 \mathrm{~J}$ and duration $1 \mathrm{~ns}$, was focused (at intensity $\sim 10^{12} \mathrm{~W} / \mathrm{cm}^{2}$ ) onto a $\mathrm{CH}$ target thus producing a background plasma. A second and third beam, with energy $1.5 \mathrm{~kJ}$ and duration $2 \mathrm{~ns}$, were then focused onto two opposing $\mathrm{CH}$ targets, leading to the production of two counter-propagating ablation plasmas. Even though the details of the resulting three plasma flows are not fully documented in either (Schaeffer et al. 2017a) or the companion paper (Schaeffer et al. 2017b), the density and temperature of the overlapping plasma were estimated to $\sim 6 \times 10^{18} \mathrm{~cm}^{-3}$ and $T_{\mathrm{e}} \sim 15 \mathrm{eV}$, respectively. The whole set-up was embedded into a $80 \mathrm{kG}$ perpendicular magnetic field produced by a pulsed current passing through Copper wires located behind the 2 opposing $\mathrm{CH}$ targets (for the readers convenience, the experimental set-up is reproduced in the left panel of Fig. 14). The resulting magnetic piston and shock structures are evidenced in the right panels of Fig. 14. The importance of the background plasma (created by the first $100 \mathrm{~J}$-beam) is made clear by comparing panel (a) to panels (b-e). Without background plasma, panel (a), no shock is observed. In the presence of a background plasma, panels (b-e), a shock-like structure is observed in all cases, strongest in the presence of the external magnetic field [panels (c-e)], but still present when no external magnetic field is applied [panel (b)]. The authors advance the possibility, supported by PIC simulations, that in this latter case, the Biermann-Battery process was responsible for seeding a large scale magnetic field even though no external one is applied. Note also, that in panel (c), the authors report the creation of a shock when only one of the $1.5 \mathrm{~kJ}$-beam was used, demonstrating that only one piston plume interacting with the background plasma was needed to produce a magnetized shock. Last, panel (f) reports the measurement obtained using proton radiography and reveals a strong magnetic field compression at the location of the shock structure. 
Note that the overall experimental campaign strongly relied on advanced diagnostics [shadowgraphy, angular filter refractometry (Haberberger et al. 2014), and proton radiography] as well as the combined used of hydrodynamic (for the plasma characterization) and PIC (for the shock formation and evolution) simulations.

Since these experiments, the effort in producing and studying magnetized shocks has continued, e.g., exploring the possibility to produce parallel shocks (Weidl et al. 2017), or to use the Biermann-Battery process to magnetize the plasmas (Umeda et al. 2019).

Weibel-mediated shocks Weibel-mediated collisionless shocks are certainly the most sought after collisionless shocks in laser-based laboratory astrophysics experiments. Even though important progress have been made in the last decade, recreating such shocks in the laboratory has not yet been achieved. The main difficulty in recreating such shocks stems from the need to achieve flow densities that are, on the one hand, sufficiently small to ensure that one operates in the collisionless regime and, on the other hand, large enough for the ion Weibel instability to develop and the resulting magnetic turbulence to build up. The combined experimental and theoretical effort in this endeavor has been started since the early 2010s and HED NIF-class laser systems, allowing to produce plasma flows with densities of a few $10^{19} \mathrm{~cm}^{-3}$ and velocities of several $1000 \mathrm{~s} \mathrm{~km} / \mathrm{s}$, have been identified as the most promising path toward collisionless Weibel-mediated shock formation.

The first step toward creating plasma flows relevant for such studies was taken by Park et al. (2012), demonstrating the possibility to drive plasma flows with velocities of $\sim 1000 \mathrm{~km} / \mathrm{s}$ and densities of $\sim 10^{18} \mathrm{~cm}^{-3}$ from plasma ablation at the Omega laser facility. The production of large-scale electromagnetic structures (Kugland et al. 2012) and later clear demonstration of Weibel-type ion filamentation instabilities (Fox et al. 2013; Huntington et al. 2015) in the presence of counter-streaming plasmas were obtained by irradiating a pair of opposing plastic $(\mathrm{CH})$ foils with few kJ, few ns laser pulses on the Omega EP laser system. Figure 15 reproduces the schematic experimental set-up used by Huntington et al. (2015) together with a typical proton radiography measurement of the magnetic field filamentary structures following from the development of the ion Weibel instability. The region imaged by the proton radiograph is about $3 \mathrm{~mm}$ wide, and the filamentary structures have a typical width of $\sim 150-300 \mu \mathrm{m}$, consistent with the ion skin-depth for the reported plasma density of a few $10^{18} \mathrm{~cm}^{-3}$.

The plasmas flows achievable at Omega are unfortunately too low density, and short life, to allow creating a Weibel-mediated collisionless shock. A recent theoretical model and 2D PIC simulations by Ruyer et al. (2016) indeed predict that the isotropization necessary for shock formation may be achievable if the two counterstreaming flows overlap over a length of the order of at least:

$$
L_{\mathrm{iso}} \simeq 35\left[m_{i} /\left(Z m_{e}\right)\right]^{0.4}\left(c / \omega_{p i}\right) \rightarrow 5 \mathrm{~cm} \times\left(\frac{A}{Z}\right)^{0.9} \sqrt{\frac{10^{19} \mathrm{~cm}^{-3}}{n_{0}}}
$$

where $c / \omega_{\mathrm{pi}}$ is the ion skin-depth associated to the plasma flow density $n_{0}, m_{\mathrm{i}}\left(m_{\mathrm{e}}\right)$ the ion (electron) mass, and $Z(A)$ the ion charge (mass) number. Conversely, the two flows shall overlap for a time of the order of $\tau_{\text {iso }}=L_{\text {iso }} / v_{0} \simeq$, with $v_{0}$ the relative flow 


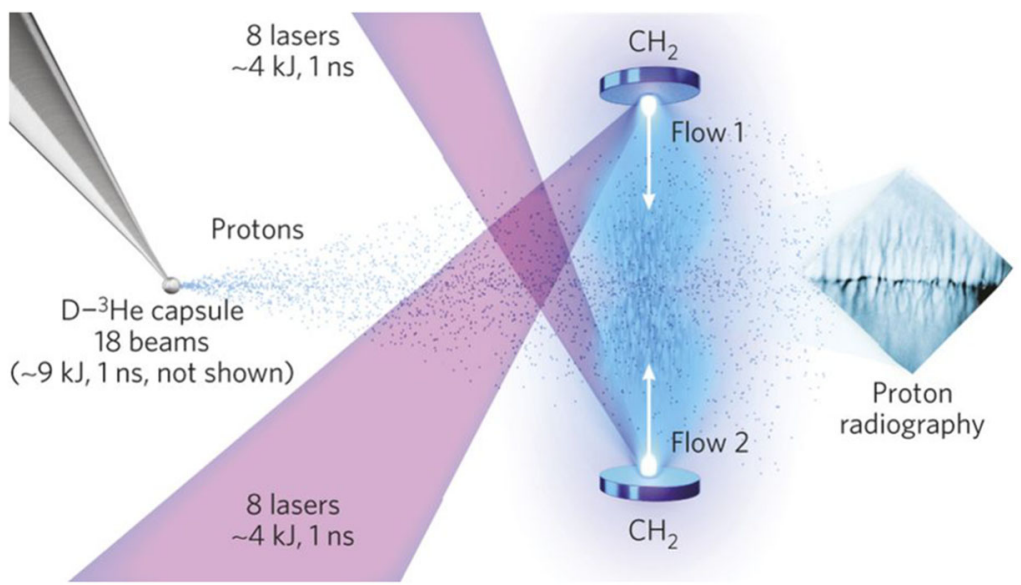

Fig. 15 Demonstration of the ion Weibel instability in counter-streaming plasmas on the Omega EP facility. Simulation setup: two ablation plasmas are formed in counter-streaming configuration by irradiating two opposing plastic targets. A ${ }^{3} \mathrm{He}-\mathrm{D}$ target is imploded to drive fusion reactions that allow for the production of $3 \mathrm{MeV}$ and $14.7 \mathrm{MeV}$ protons that are used to radiograph the electromagnetic fields developed in the region where the two counter-streaming plasma overlap. The resulting proton radiograph (here taken about 5 ns after the beginning of interaction using the $14.7 \mathrm{MeV}$ protons) shows evidence of the growth of filamentary structures associated to the Weibel magnetic fields. Image reproduced by permission from Huntington et al. (2015), copyright by Macmillan

velocity. A necessary, yet not sufficient, condition for maintaining the collisionless regime imposes that the isotropization length given by Eq. (21) is much smaller than the characteristic ion-ion collision mean-free-path (Park et al. 2012):

$$
\lambda_{\mathrm{mfp}} \simeq 5 \mathrm{~cm} \times \frac{A^{2}}{Z^{4}}\left(\frac{v_{0}}{1000 \mathrm{~km} / \mathrm{s}}\right)^{4}\left(\frac{10^{19} \mathrm{~cm}^{-3}}{n_{0}}\right),
$$

for which are considered only collisions between ions of the two counter-streaming flows. These estimates allow to infer that Weibel-mediated collisionless shocks may be achieved in the presence of (hydrogen) plasma flows with density of the order of $10^{19} \mathrm{~cm}^{-3}$, colliding at a relative velocity of at least a few $1000 \mathrm{~s} \mathrm{~km} / \mathrm{s}$, provided these flows overlap over distance of a few $\mathrm{cm}$ during a few to tens of ns. Such conditions can be met only at the most energetic, MJ-class laser systems such as NIF.

The first experiments to recreate Weibel-mediated shocks at NIF have been started in the framework of the Discovery Science program. The first experimental results were reported by Ross et al. (2017) focusing on how to tune the experimental conditions to access the collisionless regime. These experiments, for which no external magnetic field was used, considered two solid targets, made of a mixture of Carbon and either hydrogen or deuterium $(\mathrm{CH}$ or $\mathrm{CD}$ ), each irradiated by several beams allowing to deliver energies of $\sim 250 \mathrm{~kJ}$ (much larger than accessible e.g. at Omega) per foil during about $5 \mathrm{~ns}$. The resulting ablation plasma flows at velocities of $\sim 1000 \mathrm{~km} / \mathrm{s}$ and (ion) densities of a few $10^{19} \mathrm{~cm}^{-3}$ in the interaction (overlapping) region. This work demonstrated that if the foils (in opposing configuration) were sufficiently distant 
from one another, collisional effects could be strongly mitigated due to the reduction of the plasma flow density in the overlapping region. Most importantly, this work reported evidence of a collisionless (collective) heating in the flow interaction region, which the authors associate with the nonlinear stage of the Weibel instability and thus to the early stage of shock formation. This result suggests that the scientific community is on the verge of producing Weibel-mediated collisionless shocks in the laboratory. A new experiment was actually conducted at NIF in the last months increasing the driving laser beam energy to $\sim 500 \mathrm{~kJ}$ delivered to each foil. This experiment is expected to lead to shock formation, and may also gives the first signs of particle acceleration in the shock. To this date, the results of this last experimental campaign have not been announced.

Prospective numerical studies The possibility to drive collisionless shocks in the laboratory has prompted the laser-plasma community to investigate various laboratory configurations to drive collisionless collective processes and shocks in silico. Indeed, various numerical experiments have been performed using Particle-In-Cell (PIC) simulation. Even if some of these numerical experiments consider laser parameters not yet within our reach (ultra-short ps-level, energy at the 100s J level) other have addressed conditions that are or will soon be achievable on the forthcoming extreme light facilities such as Apollon or ELI.

Fiuza et al. (2012) put forward the possibility to drive-through Weibel-like instabilities - a collisionless shock in a dense target using an ultra-intense light pulse. This scenario was revisited by Ruyer et al. (2015) that demonstrated the dominant role of laser-driven hot electrons in the shock formation. More recently, Grassi et al. (2017) showed that tuning the laser-plasma interaction configuration can help mitigate the hot electron production so that shock formation can be driven by the ion Weibel instability, as expected in astrophysical scenarios.

In addition, dense electron-positron flows have been produced in laser-plasma experiments (Chen et al. 2015; Sarri et al. 2015), offering the opportunity to study pair-plasma processes in the laboratory, and motivated various numerical experiment. Using QED-PIC simulation, Lobet et al. (2015) demonstrated the possibility to drive ultra-relativistic, counter-propagating electron-positron pair plasmas using extreme light pulses (with intensity beyond $10^{23} \mathrm{~W} / \mathrm{cm}^{2}, 100 \mathrm{~s} \mathrm{~kJ}$ and duration of few tens of fs). Ultra-fast isotropization and thermalization (a first step in shock formation) were observed in the simulation, and associated to both the Weibel instability and a remarkable contribution from synchrotron emission by the ultra-relativistic leptons in the strong (Weibel) magnetic fields. More recently, the (collisionless) interaction of midly relativistic pair jets with background (electron-ion) plasma was also investigated in kinetic simulations (Dieckmann et al. 2018a, b). Remarkably, these studies are not only motivated by astrophysics (Dieckmann et al. 2019) but also by recent experiments that demonstrated the growth of a current-driven instability developing during the interaction a quasi-neutral pair beam with a background (electron-ion) plasma (Warwick et al. 2017). 

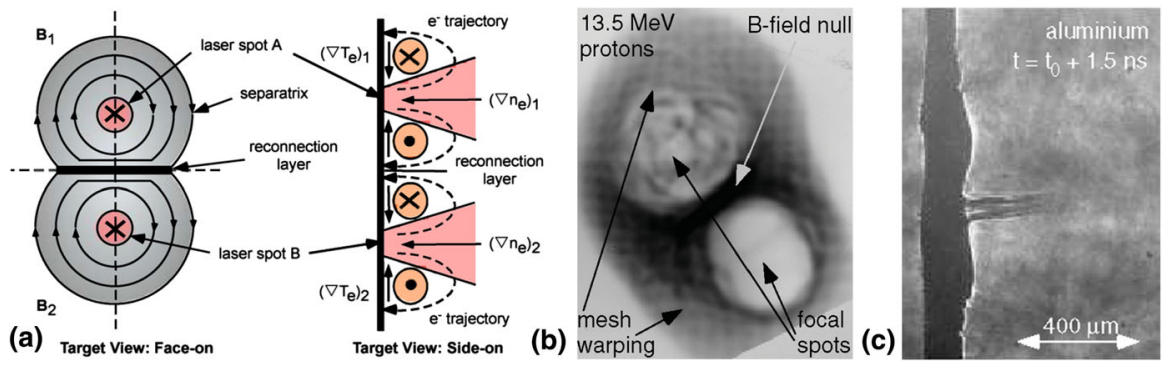

Fig. 16 Adapted from the work by Nilson et al. (2006) presenting the first magnetic reconnection experiment with laser-created plasmas. a Experimental set-up using the two-spot configuration. b Proton-radiagraphy measurements showing in dark the regions were protons are recorded. c Shadowgraphy measurements indicating the formation of two jets at the reconnection layer

\subsubsection{Magnetic reconnection experiments}

Laser-plasma experiments also provide a test bed for magnetic reconnection studies. $^{22}$ The first evidences for magnetic reconnection in a laser-plasma experiment were reported by Nilson et al. (2006). This experiment was performed at the VULCAN laser facility in the UK, and relied on a now popular set-up consisting in firing two laser pulses at a solid (here Aluminium or Gold) target (see Fig. 16a). The interaction of each pulse leads to plasma ablation and expansion associated with the generation of an azimuthal magnetic field through the Biermann-Battery process. In between the two pulses, the magnetic fields driven by the two pulses are in an anti-parallel configuration, and a reconnection layer can form.

This experiment was carried out in the HED regime of interaction, each laser pulse of the VULCAN facility delivering $200 \mathrm{~J}$ over $1 \mathrm{~ns}$ in a $30-50 \mu \mathrm{m}$ focal spot (the corresponding laser intensity is moderate $\sim 10^{15} \mathrm{~W} / \mathrm{cm}^{2}$ ). Various complementary diagnostics were used, highlighting features consistent with magnetic reconnection. (i) Proton radiography (Borghesi et al. 2001) allowed to probe the generated magnetic fields. A typical measurement is reproduced in Fig. 16b; and an additional analysis of these measurements is given in Willingale et al. (2010). Light regions correspond to regions free of protons, i.e., to regions where the strong Biermann-Battery magnetic field (estimated to be of the MG-level in this experiment few 100s of ps after the ablated plasma started expanding) is present.

The presence of a strong proton signal (dark region) in between the two lighter blobs was identified as the reconnection layer, where opposite magnetic field lines can reconnect and lead to a null-magnetic field region. (ii) In addition, the interaction region was also probed by a short (10ps) light pulse allowing to produce a shadowgraphy [as well as an interferogram (not shown here)] of the interaction region. Such a shadowgram is reproduced in Fig. 16c and shows the formation - on the ns-timescale-

\footnotetext{
22 In this review, we focus once more on laser-plasma experiments. Yet, other experiments have been conducted on various devices: the Magnetic Reconnection Experiment (MRX) at Princeton (Yamada et al. 1997), the LAPD at UCLA (Gekelman et al. 2010), Z-pinch machines (Hare et al. 2017). Magnetic reconnection is also known to affect Tokamak experiments (Goetz et al. 1991). See also Howes (2018) for a review of various laboratory experiments for space plasma physics.
} 
of two distinct jets [see original paper and Nilson et al. (2008) for more details] with velocities of $\sim 500 \mathrm{~km} / \mathrm{s}$, which would not be expected should only hydrodynamic processes govern the plasma evolution. (iii) Finally, Thomson Scattering measurements (not shown here) showed that, while the electron temperature was of the order of $800 \mathrm{eV}$ (at $1.5 \mathrm{~ns}$ ) and $700 \mathrm{eV}$ (at $2.5 \mathrm{~ns}$ ) in the ablated plasmas, a much higher electron temperature $\sim 1.7 \mathrm{keV}$ (at $1.2 \mathrm{~ns}$ ) was measured in what was identified as the reconnection layer (where there is no laser). Such high electron temperatures were also put forward as a result of magnetic reconnection; and were consistent with supporting hybrid simulations.

Since this first experiment, similar results were obtained on other HED laser facilities. Li et al. (2007) report on an experiment performed at the OMEGA laser facility using a slightly more energetic $1 \mathrm{~ns}$ laser pulse $(500 \mathrm{~J})$, with a spot diameter of $\sim 800 \mu \mathrm{m}$ (corresponding to an intensity of $\sim 10^{14} \mathrm{~W} / \mathrm{cm}^{2}$ ). This experiment benefited from a high-quality proton radiography (monoenergetic $14.7 \mathrm{MeV}$ protons were produced by fusion reactions from an imploded $\mathrm{D}^{3}$-He target), which allows the authors to probe the changes in the magnetic field topology as magnetic reconnection proceeds. See also Rosenberg et al. (2015b, a). Similarly, Zhong et al. (2010) reported on a similar experiment carried out on the Shenguang II (SG II) laser facility in Shanghai, China. In this experiment, four laser beams (1ns, few 100s J, 50-100 $\mu \mathrm{m}$-wide spots) are used to drive the plasma expansion in the two-spot configuration previously discussed, but shining the lasers on the front and back side of the target simultaneously. This experiment also put the accent on scaling their results with respect to reconnection outflows in solar flares. As an exemple, relying on X-ray imaging of the interaction region, the authors could demonstrate a change in the directionality of the jets due to an asymmetry in the driving laser intensities, ${ }^{23}$ as shown in Fig. 17.

Another experimental set-up was also proposed by Fiksel et al. (2014) and conducted on the OMEGA EP laser. In contrast with the previous experiments, this new set-up relies on (i) an head-on configuration with two targets (irradiated by kJ, ns laser beams), (ii) current-carrying conductors placed behind the two targets to create an external (up to $80 \mathrm{kG}$ ) magnetic field imposed perpendicular to the expanding plasma flows and designed such that the field a x-type null point in between the two targets; and (iii) the presence of a background plasma created by a third (100J, 1ns) laser beam. Proton radiography measurements indicate the formation and collision of magnetic ribbons, pileup of magnetic flux and reconnection which are found to be in remarkably good agreement with 2D PIC simulations (that include particle collisions).

While the previous experiments were conducted in a collisional regime, more recent experiments have focused on the collisionless regime. Dong et al. (2012) conducted an experiment on the SG II laser, using a similar set-up then previously presented by Zhong et al. (2010), but firing the laser beams (450J on each target) at two Altargets separated by 150-240 $\mu \mathrm{m}$, which, even though the collisionless nature of the reconnection region is not fully addressed, lead the authors to claim the study of a

\footnotetext{
23 See also the work by Rosenberg et al. (2015a) for a study of asymmetric reconnection OMEGA laser facility.
} 
Fig. $17 \mathrm{X}$-ray imaging showing two bright spots in the Al target were the expanding plasmas are heated by the laser beams. Here the asymmetry in the laser intensities (in regions B1 and B2) leads to an inclination of the upward flow. Bellow the Al-target, a Cu-target is placed. The downward flow impinges on this target and results in a hot $\mathrm{X}$-ray source. Image reproduced with permission from Zhong et al. (2010), copyright by

Macmillan

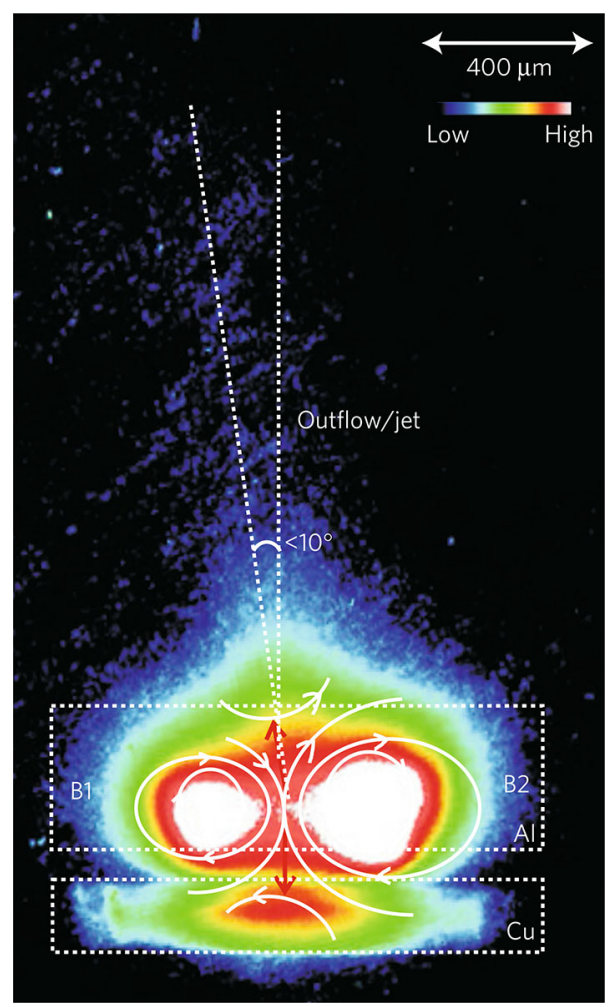

structure of collisionless reconnection. These authors also report on the ejection of a plasmoid that, when it rapidly propagates away, deforms the reconnected magnetic field and generate a secondary current sheet. This process seems to be well reproduced by PIC simulations, and the primary reconnection event is found to be associated with well-collimated plasma outflows containing high-energy $(\mathrm{MeV})$ electrons.

In addition, Raymond et al. (2018) conducted experiments, on both the OMEGA EP facility and the HERCULES laser at University of Michigan, in a regime where magnetic reconnection was not only collisionless but also driven by relativistic electrons. This was made possible by using short pulse laser beams ( $20 \mathrm{ps}$ for the OMEGA EP laser, 40 fs for the HERCULES laser) in a configuration otherwise similar to that (the two-spot experiment) initially proposed by Nilson et al. (2006). Using short pulses indeed allowed to reach ultra-high intensities $\left(\sim 10^{18} \mathrm{~W} / \mathrm{cm}^{2}\right.$ on OMEGA EP and $\sim 210^{19} \mathrm{~W} / \mathrm{cm}^{2}$ on HERCULES), thus allowing to enter the relativistic regime of laser-plasma interaction. Figure 18 shows the typical experimental set-up as well as a typical X-ray imaging where the two heated and expanding plasmas can be seen, together with a reconnection layer in between. In addition, the authors report the formation of a non-thermal (few $\mathrm{MeV}$ ) electron population whenever reconnection is expected, consistent with supporting 3D PIC simulations. 


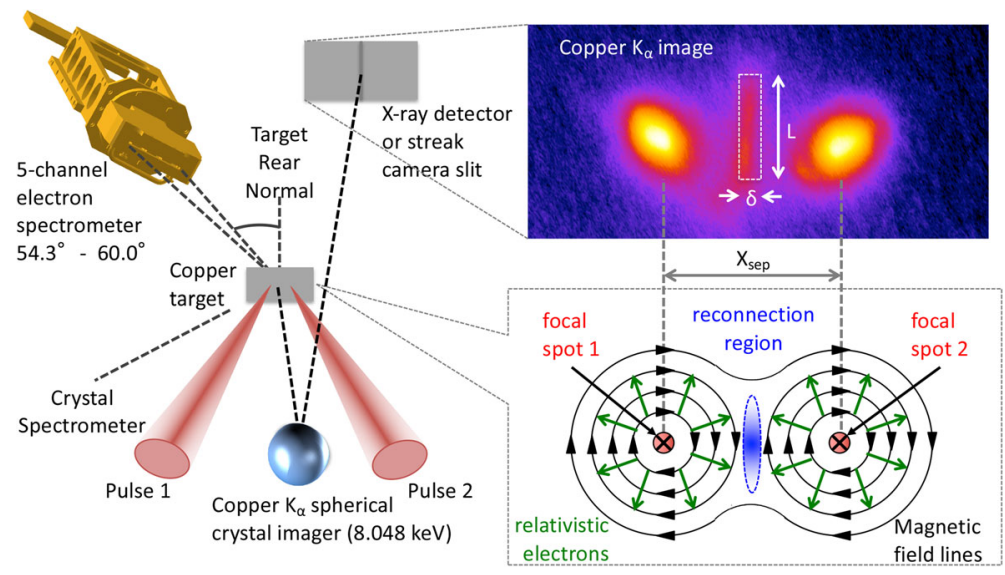

Fig. 18 Experimental set-up relying on ultra-high-intensity short pulse laser beams allowing to probe relativistic reconnection. A typical X-ray imaging shows the location of the two hot expanding plasmas and, in between, the reconnection layer. Image reproduced with permission from Raymond et al. (2018), copyright by APS

\section{Solving kinetic problems}

This section reviews the model equations used to describe particle kinetic physics, i.e., the dynamics of charged particles in the configuration and momentum space under the effect of electro-magnetic forces. The section is divided as follows: in Sect. 3.1 we describe the Vlasov-Maxwell system of equations, then in Sect. 3.2 we discuss the numerical methods developed to follow the dynamics of such a system. Section 3.3 discusses the particle-in-cell (PIC) technique used to study solutions of the VlasovMaxwell system. In Sect. 3.4 we provide a discussion on the comparison between PIC and Vlasov approaches. Section 3.5 briefly describes hybrid methods where a fluid approximation is introduced for the electronic component whereas kinetic (PIC) techniques are used to describe ions. In Sect. 3.6 we specifically discuss the FokkerPlanck description of kinetic problems. The Fokker-Planck approach is particularly well-adapted to investigate cosmic ray propagation. Finally, we give a particular focus on Fokker-Planck simulations developed in the context of the study of the radiative transfer in hot plasmas around compact objects.

\subsection{The Vlasov-Maxwell description of a collisionless plasma}

\subsubsection{Governing equations}

Let us consider a plasma composed of various species, labeled $s$, corresponding to particles with mass $m_{s}$ and charge $q_{s}$. The kinetic description of this plasma relies on the representation of each species $s$ by its (one-particle) distribution function $f_{s}(t, \mathbf{x}, \mathbf{p})$, $f_{s}(t, \mathbf{x}, \mathbf{p}) d^{3} x d^{3} p$ measuring, at any time $t$, the (probable) number of particles of species $s$ in a volume element $d^{3} x d^{3} p$ at a position $(\mathbf{x}, \mathbf{p})$ in phase-space (x and $\mathbf{p}$ 
standing for the spatial and momentum coordinates, respectively). In the absence of collisions, the evolution of the distribution functions $f_{s}$ satisfies the Vlasov equation:

$$
\frac{\partial}{\partial t} f_{s}+(\mathbf{v} \cdot \nabla) f_{s}+\left(\mathbf{F}_{s} \cdot \nabla_{p}\right) f_{s}=0
$$

where $\mathbf{v}=\mathbf{p} /\left(m_{s} \gamma\right)$ is the velocity corresponding to a particle of momentum $\mathbf{p}$ and Lorentz factor $\gamma=\sqrt{1+\mathbf{p}^{2} /\left(m_{s} c\right)^{2}}$ ( $c$ is the speed of light in vacuum), and $\mathbf{F}_{s}$ is the force acting on the species particles. As this work focuses on electromagnetic plasmas, this force is the Lorentz force exerted by the collective electric $\mathbf{E}$ and magnetic $\mathbf{B}$ fields:

$$
\mathbf{F}_{s}=q_{s}\left(\mathbf{E}+\frac{1}{c} \mathbf{v} \times \mathbf{B}\right) .
$$

It is important to stress that, in the Vlasov equation, the electromagnetic fields are collective fields, also referred to as macroscopic fields in the sense that they do not account for the microscopic variations developing at the particle scale. Hence collisions are not considered in this description.

The electric $\mathbf{E}(\mathrm{t}, \mathbf{x})$ and magnetic $\mathbf{B}(\mathrm{t}, \mathbf{x})$ fields are in general functions of both space and time, and satisfy Maxwell's equations ${ }^{24}$ :

$$
\begin{aligned}
\nabla \cdot \mathbf{E} & =4 \pi \rho, \\
\nabla \cdot \mathbf{B} & =0, \\
\nabla \times \mathbf{E} & =-\frac{1}{c} \frac{\partial \mathbf{B}}{\partial t}, \\
\nabla \times \mathbf{B} & =\frac{4 \pi}{c} \mathbf{J}+\frac{1}{c} \frac{\partial \mathbf{E}}{\partial t} .
\end{aligned}
$$

The electromagnetic fields act onto the plasma through the Lorentz force (24), and are in turn modified by the plasma through the total charge and current densities, $\rho=\sum_{s} \rho_{s}$ and $\mathbf{J}=\sum_{s} \mathbf{J}_{s}$, respectively, where each species charge and current densities are defined as:

$$
\begin{aligned}
& \rho_{s}(t, \mathbf{x})=q_{s} \int d^{3} p f_{s}(t, \mathbf{x}, \mathbf{p}), \\
& \mathbf{J}_{s}(t, \mathbf{x})=q_{s} \int d^{3} p \mathbf{v} f_{s}(t, \mathbf{x}, \mathbf{p}) .
\end{aligned}
$$

The coupled system of Eqs. (23) and (25), together with the Lorentz force (24) and definitions of the charge and current densities (26) form the Vlasov-Maxwell model. It provides a self-consistent, kinetic description for the evolution of a collisionless plasma and the associated collective electromagnetic fields.

\footnotetext{
${ }^{24}$ In some cases where only electrostatic fields are important, only Poisson Eq. (25a) may be used. The Vlasov-Poisson description is of particular importance for the description of cold plasmas in particular, as considered e.g., for plasma propulsion, see Ref. (Boeuf and Garrigues 2018).
} 


\subsubsection{Initial and boundary conditions}

The Vlasov-Maxwell model relies on a system of partial differential equations and thus requires initial and boundary conditions. The initial condition of the system (at time $t=0$ ) consists first in defining the initial distribution functions $f_{s}(t=0, \mathbf{x}, \mathbf{p})$ for all species $s$ of the system. One usually considers equilibrium ${ }^{25}$ distribution functions, and Maxwellian or Maxwell-Jüttner distribution functions (drifting or not) are often considered. ${ }^{26}$ The initial electromagnetic fields spatial distribution $\mathbf{E}(t=0, \mathbf{x})$ and $\mathbf{B}(t=0, \mathbf{x})$ also needs to be prescribed. At $t=0$, these fields have to satisfy Eq. (25a) and Eq. (25b), respectively. Hence, $\mathbf{B}(t=0, \mathbf{x})$ has to be divergence free, while $\mathbf{E}(t=0, \mathbf{x})$ can be either divergence free (e.g., if an external electric field is considered) or has to be computed from Poisson's Eq. (25a) using the initial distribution functions $f_{s}(t=0, \mathbf{x}, \mathbf{p})$ to compute the initial charge density.

Various boundary conditions (BCs) can be considered and will strongly depend on the physics at hand. First, BCs on the distribution functions can be used to reflect, thermalize already existing particles or inject new particles at the border of the spatial domain. In addition, when directly solving the Vlasov equation in phase-space (see Sect. 3.4 on so-called Vlasov codes), BCs on the momentum component have to be considered. Similarly, electromagnetic fields can be reflected, absorbed or injected at the domain border by prescribing the correct $\mathrm{BCs}$ for the electric and magnetic fields.

\subsection{Solving the Vlasov-Maxwell system numerically: general considerations}

Computer simulation is an outstanding tool for solving the Vlasov-Maxwell system of equations together with the prescribed initial and boundary conditions, and most of today's kinetic simulations of plasmas rely on massively parallel tools to do so. In what follows, we present two of the main numerical approaches to solve this system. The first method is used in so-called Vlasov codes, while the second is used in so-called Particle-In-Cell (PIC) codes. The main difference between the two methods lies in the way they solve the Vlasov equation. Otherwise, both methods follow the same procedure which rely on discretizing the fields onto a spatial grid (henceforth referred to as the simulation grid), advancing the distribution function and then updating the associated charge and current densities onto the simulation grid. This procedure is here briefly detailed and summarized in Fig. 19.

\subsubsection{Initialization and time-loop}

First, the initialization step consists in prescribing the distribution functions for all species $s$ at time $t=0$ together with the initial electric and magnetic fields. Again, one should stress that both fields must satisfy Eqs. (25a) and (25b). Thus the initial electric field can be obtained by solving Poisson Eq. (25a) and adding any divergencefree (e.g., external) electric field. For the magnetic field, one can start either from a

\footnotetext{
25 At least in the sense of hydrodynamic equilibrium.

26 The loading of a species with drifting Maxwell-Jüttner distribution in Particle-In-Cell codes should be handle with some care, as discussed e.g., in Refs. (Melzani et al. 2013; Zenitani 2015).
} 


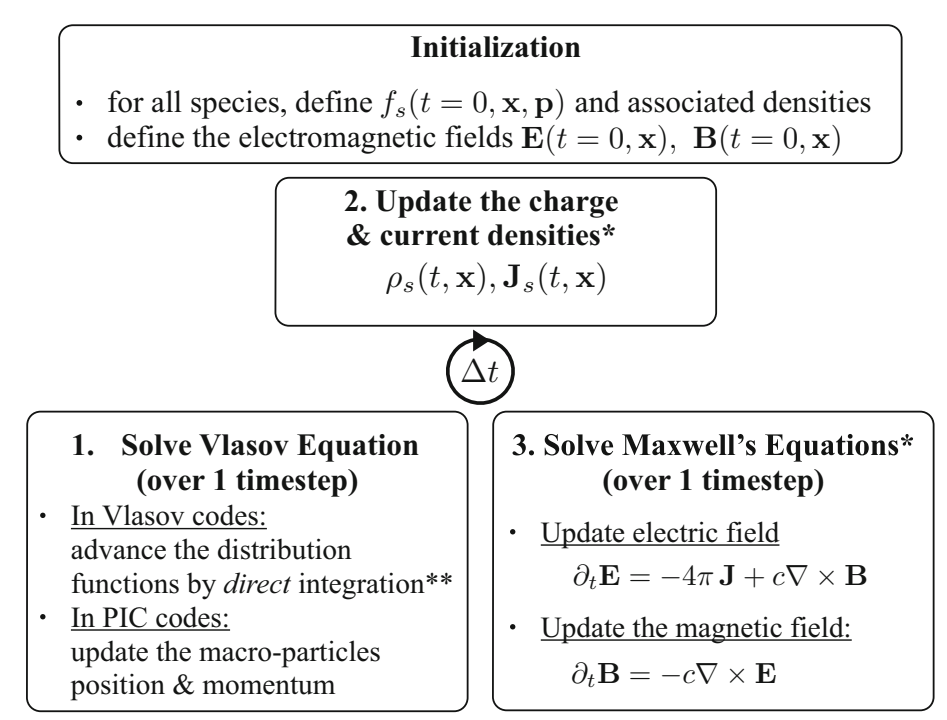

Fig. 19 Schematic presentation of the numerical procedure used to solve the Maxwell-Vlasov system of equations. *If the computation of the current densities onto the simulation grid is done in such a way that charge is conserved, and considering that the initial electromagnetic fields satisfy Eqs. (25a) and (25b), solving Maxwell-Ampère (Eq. (25d)) and Maxwell-Faraday (Eq. 25c) is sufficient to ensure that Eqs. (25a) and (25b) remain satisfied (within the machine precision) at all times. **Direct integration here refers to advancing the distribution function on a grid in phase-space $(\mathbf{x}, \mathbf{p})$, as further discussed in Sect. 3.4

zero magnetic field or any non-zero divergence-free magnetic field that will act as an external field applied to the system.

One then enters the time loop of the numerical solver. This time loop consists in advancing the various quantities (defined on the simulation grid) from a timestep $n$ (time $t_{n}=n \Delta t$ ) to the next timestep $n+1$ (time $t_{n+1}=t_{n}+\Delta t$ ). Various methods are available to do so, some of which rely on defining different quantities at either integer or half-integer timesteps to ensure a centering of the numerical time derivatives. For the sake of simplicity, we will not account for this subtlety here.

The first step in the time-loop consists in advancing the distribution functions for all species $s$. Knowing the electromagnetic field at timestep $n$, the distribution functions at timestep $n+1$ are computed either by direct integration (this is the case in Vlasov codes, see Sect. 3.4) or by advancing so-called macro-particles which are in effect discrete element of the distribution function (this is the case in PIC codes, as described in Sect. 3.3).

The updated distribution functions are then used to compute, onto the simulation grid, the updated charge and current densities (step 2).

These densities are then used, in a third step, to advance the electromagnetic fields from time step $n$ to $n+1$. If the current density deposition onto the grid (step 2) conserves the charge ${ }^{27}$ solving Maxwell-Ampère and Maxwell-Faraday Eqs. (25d) and $(25 \mathrm{c})$, respectively, is sufficient to ensure that the electromagnetic fields remain

$\overline{27}$ In the sense that it satisfies, at the machine precision, the continuity equation $\partial_{t} \rho+\nabla \cdot \mathbf{J}=0$. 
Maxwell-consistent. To solve these equations, different Maxwell solvers are available (and are discussed in Sect. 3.2.2).

With the fields updated, the loop gets back to step 1 and is run as long as required to reach the final timestep of the simulation.

\subsubsection{Brief discussion of Maxwell solvers}

Various numerical methods (so-called solvers) can be used to solve Maxwell's equations. Here we briefly introduce two methods that are most popular in plasma simulation.

The Finite-Difference Time-Domain (FDTD) method is a time-honoured approach to solve Maxwell's equations (Taflove 2005). It relies on a finite-difference discretization of the partial time derivatives and curl operators in Maxwell-Ampère and Maxwell-Faraday's equations. Most important is that all differential operators are centered. Centering in space requires the use of a staggered grid, the so-called Yee grid, different components of the electric and magnetic fields being defined at different positions in space (onto the grid). Centering in time requires that the electric and magnetic fields are advanced in a leap-frog way, e.g., solving first Maxwell-Ampère equation to advance the electric field then using the updated electric field to solve Maxwell-Faraday's equation and advance the magnetic field. A major advantage of the FDTD method stems from its local nature, which allows for its easy and effective (scalable) implementation in massively parallel environments. A major drawback of the method is that it is subject to numerical dispersion, the numerical electromagnetic wave propagating with velocities potentially smaller than $c$ (Birdsall and Langdon 1985; Nuter et al. 2014). This effect is in part responsible for the spurious numerical Cherenkov instability, see Sect. 3.3.3.

Pseudo-Spectral methods, on the other hand, allow to solve Maxwell's equations with an extraordinary level of precision and correctly capture the dispersion relation of electromagnetic waves. They consist of advancing the electromagnetic fields in Fourier space (for the spatial coordinates) while relying on an (explicit) finite-difference for the time derivatives (Liu 1997; Vay et al. 2013).

The increased precision allowed by pseudo-spectral methods however comes with the cost of global communications associated with the use of Fourier transforms over the entire simulation domain. These global communications have been a major impediment to the adoption of pseudo-spectral methods in massively parallel environments. Recently, Vay et al. (2013) have proposed a domain-decomposition method that allows for the efficient parallelization of pseudo-spectral solvers. This method takes advantage of the finiteness of the speed of light and relies only on local (over subdomains much smaller than the entire simulation domain) fast Fourier transforms and communications between neighbouring subdomains. Vincenti and Vay (2018) have demonstrated that this method may allow for unprecedented scalability of pseudo-spectral solvers over tens to hundreds of thousands of computing elements (cores). 


\subsection{Particle-In-Cell codes}

The Particle-In-Cell (PIC) method was introduced in the mid-1950s by Harlow and collaborators to solve fluid dynamics problems (see Harlow 2004 and references therein). Following the pioneering works of Buneman (1959), Dawson (1962), Birdsall and Fuss (1969) and Langdon and Birdsall (1970) (see Dawson 1983 and Verboncoeur 2005 for a history of the development of PIC codes), PIC codes have become a central tool for plasma simulation (Birdsall and Langdon 1985). Indeed, the simplicity of the PIC method together with the possibility to implement it efficiently in a massively parallel environment have established PIC codes as the most popular tool for the kinetic simulation of plasmas.

\subsubsection{Method}

The PIC method differs from the Vlasov-code approach in the way it solves the Vlasov equation, and by extension, the way it computes the current densities on the simulation grid. In PIC codes, the distribution function $f_{S}$ is approximated as a sum over $N$ macroparticles:

$$
f_{S}(t, \mathbf{x}, \mathbf{p}) \equiv \sum_{p=1}^{N} w_{p} S\left(\mathbf{x}-\mathbf{x}_{p}(t)\right) \delta\left(\mathbf{p}-\mathbf{p}_{p}(t)\right),
$$

where $\delta(\mathbf{p})$ is the Dirac delta-distribution, $S(\mathbf{x})$ is the so-called particle shape-function, and $w_{p}, \mathbf{x}_{p}(t)$ and $\mathbf{p}_{p}(t)$ are the $p^{t h}$ particle numerical weight, position and momentum, respectively. The macro-particles can be regarded as walkers in Monte-Carlo simulations, and the PIC method as a Monte-Carlo procedure for solving the Vlasov equation (Lapeyre et al. 2003). The initial state of each species of the plasma is obtained from a random sampling of the distribution function $f_{s}$ at time $t=0$, and the Vlasov equation is then solved following the macro-particles/walkers motion through the influence of the collective electromagnetic fields.

Indeed, introducing the discretized distribution function (27) in Vlasov equation (23), one can show (see, e.g., Derouillat et al. 2018) that solving Vlasov equation reduces to solving, for all macro-particles $p$, their equations of motion:

$$
\begin{aligned}
\frac{d \mathbf{p}_{p}}{d t} & =\frac{q_{s}}{m_{s}}\left(\mathbf{E}_{p}+\frac{\mathbf{v}_{p}}{c} \times \mathbf{B}_{p}\right), \\
\frac{d \mathbf{x}_{p}}{d t} & =\mathbf{v}_{p}=\frac{\mathbf{p}_{p}}{m_{s} \gamma_{p}},
\end{aligned}
$$

with $\gamma_{p}=\sqrt{1+\mathbf{p}_{p}^{2} /\left(m_{s} c\right)^{2}}$ the $p^{t h}$ macro-particle Lorentz factor and where we have introduced the electric and magnetic fields seen by the macro-particle: 


$$
\begin{aligned}
& \mathbf{E}_{p}=\int d^{3} x S\left(\mathbf{x}-\mathbf{x}_{p}\right) \mathbf{E}(\mathbf{x}), \\
& \mathbf{B}_{p}=\int d^{3} x S\left(\mathbf{x}-\mathbf{x}_{p}\right) \mathbf{B}(\mathbf{x}) .
\end{aligned}
$$

Correspondingly, the species charge and current densities on the simulation grid can be obtained by direct deposition onto the grid. Yet, such a direct deposition would not in general satisfy the charge conservation equation, and the electric fields then would required to be corrected to ensure that they verify the Poisson equation (Mardahl and Verboncoeur 1997). Some current deposition strategies that conserve the charge have, however, been proposed. A popular charge conserving deposition scheme has been proposed by Esirkepov (2001) for PIC code relying on the FDTD Maxwell solver. The macro-particle equations of motion Eq. 28 are most commonly solved using Boris pusher (Birdsall and Langdon 1985). It is a second-order leap-frog integrator where the updated particle momentum is computed knowing the electromagnetic fields at the position of the macro particle as:

$$
\mathbf{p}_{p}^{\left(n+\frac{1}{2}\right)}=\mathbf{p}_{p}^{\left(n-\frac{1}{2}\right)}+\frac{q_{s}}{m_{s}} \Delta t\left[\mathbf{E}_{p}^{(n)}+\frac{\mathbf{v}_{p}^{\left(n+\frac{1}{2}\right)}+\mathbf{v}_{p}^{\left(n-\frac{1}{2}\right)}}{2 c} \times \mathbf{B}_{p}^{(n)}\right]
$$

and the updated particle position is computed as:

$$
\mathbf{x}_{p}^{(n+1)}=\mathbf{x}_{p}^{(n)}+\Delta t \frac{\mathbf{p}_{p}^{\left(n+\frac{1}{2}\right)}}{\gamma_{p}}
$$

Several alternative solvers where recently developed (e.g., Vay 2008; Higuera and Cary 2017) presenting sometimes better accuracy than the traditional Boris algorithm.

\subsubsection{Additional physics modules}

In their most basic implementation (detailed above), PIC codes describe collisionless plasmas through the self-consistent evolution of the particle distribution functions and collective (macroscopic) electromagnetic fields. Additional physics modules can easily be implemented in PIC codes to account for additional processes. Here we provide some references for some of this processes: field ionization (Nuter et al. 2011), collisions and collisional ionization (Nanbu 1997; Nanbu and Yonemura 1998; Pérez et al. 2012), high-energy photon (synchrotron or inverse Compton) emission and its back-reaction (Duclous et al. 2011; Lobet et al. 2016; Niel et al. 2018), pair production in a strong electromagnetic [Breit-Wheeler process (Duclous et al. 2011; Lobet et al. 2016)] or Coulomb [Trident and Bethe-Heitler processes (Martinez 2018)]. These later processes are of outmost importance for extreme plasma physics as will soon be encountered on multi-petawatt laser facilities (see, e.g., Sect. 2.6.1), but also at play in the most extreme astrophysical environments around, e.g., neutron stars and black holes (Uzdensky et al. 2019). 


\subsubsection{Stability issues: relativistic flows}

It is worth mentioning that there is one important numerical issue when one deals with relativistic flows in PIC simulations: the spurious Cherenkov instability (e.g., Godfrey 1974). This instability results from the resonance of the light-wave modes with the streaming beam when electromagnetic fields are defined on a discrete Eulerian grid. As the numerical light-wave mode is affected by the finite-difference scheme, especially at high- $k$ (Birdsall and Langdon 1985), this resonance is nonphysical. The instability appears as well in spectral codes but has a different signature (Godfrey and Vay 2015). It is practically very difficult to avoid in long-term simulations of relativistic flows or shocks. Nevertheless various methods have been proposed that allow to mitigate, or at least delay the onset of the instability. Some of these methods rely on digital filtering of electromagnetic fields and/or current densities (Greenwood et al. 2004; Vay et al. 2011); modifying the numerical stencil of the FDTD solver (Lehe et al. 2013; Grassi 2017) or upgrading to a semi-implicit scheme (Pukhov 2019); special patching of the most unstable modes (e.g., Godfrey and Vay 2014; Li et al. 2017); artificially increasing the speed of light in the Maxwell solver (Nuter and Tikhonchuk 2016) or solving the PIC equations in Galilean coordinates (Lehe et al. 2016). Yet, there is no definitive solution to remove it completely. Even if the most unstable modes are 'cleaned' or stabilized, the difficulty arises in long term evolution from coupling of the secondary aliasing modes with low-wavenumber oblique modes of the streaming plasma, that is very hard to remove without touching important physical scales (Dieckmann et al. 2006). Despite this difficulty, several studies managed to push simulations beyond $10^{4} \omega_{p i}^{-1}$ allowing to extract important results from simulations (see Sect. 4).

\subsubsection{Examples}

Various PIC codes are today available and used for astrophysics or space plasma applications. ${ }^{28}$ Some of these codes are freely distributed under free-software licenses, this is the case of EPOCH ${ }^{29}$ (Arber et al. 2015) PICCANTE ${ }^{30}$ (Sgattoni et al. 2015), SMILEI, ${ }^{31}$ (Derouillat et al. 2018) TRISTAN- MP ${ }^{32}$ (Spitkovsky 2005), and ZELTRON ${ }^{33}$ (Cerutti et al. 2013). Among other proprietary codes used for astrophysics applications are A-PART (Melzani et al. 2013), CALDER (Lefebvre et al. 2003), OSIRIS (Fonseca et al. 2002), and Photon-Plasma (Haugbølle et al. 2013). Finally, other PIC codes rely on more advanced numerical schemes; e.g., the implicit code IPIC3D (Markidis et al. 2010) or the SLURM code for modeling magnetized fluids or plasmas (Olshevsky et al. 2019).

\footnotetext{
28 We restrict our presentation to electromagnetic PIC codes that have been applied to astrophysics and/or space plasma physics studies.

29 https://gitlab.com/arm-hpc/packages/wikis/packages/EPOCH.

30 https://github.com/ALaDyn/piccante.

31 www.maisondelasimulation.fr/smilei.

32 https://github.com/ntoles/tristan-mp-pitp.

33 http://ipag-old.osug.fr/ ceruttbe/Zeltron/index.html.
} 


\subsection{Vlasov-Maxwell codes}

\subsubsection{PIC codes versus Vlasov codes}

Particle-In-Cell codes reduce the problem of solving Vlasov equation to solving the (ordinary differential) equations of motion of many macro-particles. It follows that the main advantages of the PIC method are its conceptual simplicity, its robustness and easy implementation on (massively) parallel super computers. The simplicity of the PIC method also allows for PIC codes to be multi-purpose simulation tools: a single PIC code can address various problems from basics plasma physics, astrophysics studies to the modelling of laser-plasma experiments.

However, due to the introduction of a finite number of macro-particles, PIC simulation suffers from the highly exaggerated level of noise. This well known short-coming of the PIC method makes it less adapted to treating problems for which regions of phase-space where the distribution function assumes small values (e.g., in its tail) can impact the physics at play.

In contrast, Vlasov codes which directly integrate the (partial differential) Vlasov equation on a grid in phase-space are virtually noise-free, and are thus an interesting alternative to PIC codes whenever low noise simulation is required. ${ }^{34}$ The fine description allowed by Vlasov codes however comes with the cost of increased numerical complexity. As a result, Vlasov codes are in general much less multi-purpose than PIC codes, and are usually developed to tackle a definite class of problems.

\subsubsection{The problem of filamentation in phase-space}

One impediment in the development and adoption of Vlasov codes is their computational cost and memory requirement when dealing with all 6 dimensions of phase-space. This problem can however be mitigated by reducing the number of dimensions e.g., by relying on conservation laws and symmetries of the problem (see, e.g., Manfredi et al. 1995; Feix and Bertrand 2005). It also becomes less exacting with the fast development of modern high-performance computing.

A more stringent limitation to the development of Vlasov codes stems from the numerical effort necessary to directly solve the Vlasov equation, and to the problem of filamentation in phase-space in particular. Indeed, the time evolution of the distribution function in the Vlasov equation is associated with its breaking-filamentation - into increasingly small structures in phase-space, and thus to strong gradients of the distribution function. When discretizing the distribution function onto a grid with finite resolution, handling these gradients becomes numerically inaccurate, and can lead to spurious oscillations, numerical instabilities and inaccurate rendering of conserved quantities (e.g., non-positive distribution functions). Dealing with this issue greatly contributes to the numerical complexity behind Vlasov codes' development.

\footnotetext{
34 A theoretical discussion on the relative efficiency of the PIC and (direct) Vlasov approaches to treat a given problem is presented in (Feix and Bertrand 2005).
} 


\subsubsection{Example of different methods for electromagnetic Vlasov codes}

The numerical complexity of Vlasov codes has-since the seminal work by Cheng and Knorr (1976) introducing the time-splitting technique ${ }^{35}$-led to the development of various techniques to directly integrate the Vlasov equation onto a grid in phase-space. It is thus beyond the scope of this brief review to detail these techniques and we here restrict our presentation to some electromagnetic Vlasov codes and their applications. Review articles by (Filbet and Sonnendrücker 2003; Büchner 2007; Ghizzo et al. 2009; Palmroth et al. 2018) discuss various techniques, that range from finite-volume type methods (Fijalkow 1999; Filbet et al. 2001), to semi-Langrangian methods (Sonnendrücker et al. 1999), and spectral methods (Klimas 1987).

Each method has its own advantages and limitations, and Vlasov codes are usually designed to tackle a specific class of physical problems. Ghizzo et al. (1990) for instance developed a relativistic electromagnetic 1D Vlasov code to study stimulated Raman scattering; while Shoucri et al. (2015) considered the problem of stimulated Brillouin scattering. These codes actually used the conservation of canonical momentum to reduce the number of dimension in velocity/momentum-space. ${ }^{36}$ A somewhat similar approach was used to study the breaking of a relativistic Langmuir wave (Grassi et al. 2014) as well as laser-driven (electrostatic) shock acceleration of ions and ion turbulence (Grassi et al. 2016).

A (non-relativistic) Eulerian Vlasov-Maxwell solver was developed by Mangeney et al. (2002). It was applied to various studies ranging from wave propagation in magnetized plasmas (Califano and Lontano 2003), to the study of the nonlinear kinetic regime of the Weibel instability (Califano et al. 2002). An off-spring of this solver is the hybrid (kinetic ions, fluid electrons) Vlasov code (Valentini et al. 2007) used in particular to tackle turbulence studies in either 2D3V (see, e.g., Cerri et al. 2017) and 3D3V (see, e.g., Cerri et al. 2018) geometries.

Another Eulerian Vlasov-Maxwell model was developed by Umeda et al. (2009) and applied to the study of various instabilities, such as the Kelvin-Helmholtz instability (Umeda et al. 2014) or the collisionless Rayleigh-Taylor instability (Umeda and Wada 2016).

The semi-Lagrangian method introduced by Sonnendrücker et al. (1999) (see also Crouseilles et al. 2010) has also led to a new kind of Vlasov codes. As an example, a relativistic semi-Lagrangian Vlasov-Maxwell solver (VLEM) was recently developed by Sarrat et al. (2017). It was used to tackle problems related to streaming instabilities in plasmas, such as the current Weibel-filamentation and two-stream instabilities; and operates in $1 \mathrm{D} 3 \mathrm{~V}, 2 \mathrm{D} 2 \mathrm{~V}$ and $2 \mathrm{D} 3 \mathrm{~V}$ geometries.

\subsection{Hybrid methods}

In this approach thermal electrons are taken to be a massless, neutralizing and are treated as a magnetized fluid. Ions (thermal or even non-thermal) are treated using a

\footnotetext{
35 The time-splitting technique separates advection in (real) space and velocity-space.

36 Other methods have been developed that take advantage of the existence of canonical invariants to solve the Vlasov equation; see e.g., Liseikina et al. (2004), Inglebert et al. (2011).
} 
PIC approach. The advantage of this method is to eliminate Debye-scale physics while still catching microscopic phenomena.

In hybrid codes, ion positions are advanced using the Boris pusher as in PIC codes (see Sect. 3.3.1). Electron dynamics is the one of a massless fluid then

$$
m_{\mathrm{e}} n_{\mathrm{e}} \frac{d \mathbf{v}_{\mathrm{e}}}{d t}=0=-e n_{\mathrm{e}}\left(\mathbf{E}+\frac{\mathbf{v}_{\mathrm{e}}}{c} \times \mathbf{B}-\nabla \cdot \overline{\bar{P}}_{\mathrm{e}}\right) .
$$

This combined with the Ampère law for a non-relativistic flow, hence neglecting the displacement current leads to an equation for the electric field

$$
\mathbf{E} \simeq-\frac{\mathbf{v}_{\mathrm{i}}}{c} \times \mathbf{B}-\frac{1}{e n_{\mathrm{e}}} \nabla \cdot \overline{\bar{P}}_{\mathrm{e}}-\frac{1}{4 \pi q_{\mathrm{i}} n_{\mathrm{i}}}(\nabla \times \mathbf{B}) \times \mathbf{B},
$$

where $\overline{\bar{P}}_{\mathrm{e}}$ is the electron pressure rank 2 tensor. This method will not be reviewed here, the interested reader is invited to read recent references on the subject: Lipatov (2002), Kunz et al. (2014).

We note here the case of the DHYBRID code (Gargaté et al. 2007). This code is explicit fully parallelized code and it uses MPI. DHYBRID solves the dynamics of nonthermal particles based on a PIC approach. The code has been used in the context of particle acceleration and transport at collisionless shocks, some of its results are presented in Sect. 4.1.

\subsection{Solving Fokker-Planck problems}

The Fokker-Planck equation (FPE) is one of the most important equation in kinetic physics. It describes the evolution in the phase space of the particle distribution function $f(\mathbf{r}, \mathbf{p}, t)$ under the effect of a diffusion process with small increments in which initial conditions are lost (a.k.a. a Markov process). In this review we are interested in collisionless plasmas, in that case, particle diffusion results from the process of scattering off plasma waves. However, note that FPEs are also well studied in the context of collisional plasmas. We refer the interested reader to Wang et al. (2008) for the description of numerical treatments of the collision operator. As is concerning highenergy particles, the FPE describes processes which develop over scales explored by these particles, it is also adapted to the study of macroscopic processes in astrophysical plasmas detailed in Sect. 5. The interested reader can advantageously consult Risken (1989) for an overview of the properties of the FPE.

For a system of energetic particles in a magnetic field oriented along the $\mathrm{z}$ axis, we can write the FPE as (Schlickeiser 2002):

$$
\partial_{t} f+v \mu \partial_{z} f-\epsilon \Omega_{\mathrm{s}} \partial_{\phi} f=\frac{1}{p^{2}} \partial_{\mathrm{x}}\left[p^{2}\left(D_{\mathrm{xx}} \partial_{\mathrm{x}} f+a f\right)\right]+q(\mathbf{r}, \mathbf{p}, t)
$$

where the diffusion process runs over the variables: $\mathrm{x}, \mathrm{y}, \mathrm{z}, \mathrm{p}, \mu, \phi$, hence we have 25 diffusion coefficients $D_{\mathrm{xx}},{ }^{37}$ and $\mu$ and $\phi$ are the particle pitch-angle cosine and

\footnotetext{
37 The Cartesian coordinates $\mathrm{x}, \mathrm{y}, \mathrm{z}$ mark here the position of the particle's guiding center.
} 
azimuthal gyration angle respectively. Here, particles of charge $q$ and mass $m$ are relativistic (with speeds $v \simeq c$ ) and gyrate around a magnetic field of strength $\mathrm{B}$ with a gyrofrequency $\Omega_{\mathrm{s}} \simeq c / r_{\mathrm{L}}$. We note $\epsilon=q / \operatorname{sgn}(q)$. The term $a(p, \mathbf{r}, t)$ describes the momentum change of the particle either due to loss or acceleration and $q(\mathbf{r}, \mathbf{p}, t)$ represents particle injection and/or escape. Although it should be kept in mind that the FPE is deduced from the more general Vlasov equation, we focus below on numerical solutions of this equation. Often, in the context of CR physics, the FPE is not directly solved but rather the convection-diffusion equation (CDE). The $\mathrm{CDE}$ results from the former by an averaging procedure over $\phi$ and $\mu$ in the case fast scattering processes build a gyrotropic and an isotropic distribution.

\subsubsection{The Fokker-Planck equation}

We start by studying 1D diffusion problems as is the case for stochastic acceleration. In that case the FPE can be simplified as

$$
\partial_{t} F(p, t)=\frac{1}{p^{2}} \partial_{\mathrm{p}}\left[p^{2}\left(D_{\mathrm{pp}} \partial_{p} F+a(p) F\right)\right]-\frac{F}{\tau_{\mathrm{esc}}}+Q(p, t) .
$$

Here, the particle distribution $F(p, t)=\int f(\mathbf{r}, \mathbf{p}, t) \mathrm{d}^{3} \mathbf{r} \mathrm{d} \mu \mathrm{d} \phi$ is averaged over the space volume and is assumed to be isotropic (it fulfills the diffusion-convection limit) and diffusive escape is treated by the means of an escape timescale $\tau_{\text {esc }}(p)$, the loss/gain $a(p, t)$ term is also averaged. This equation can be solved using finite difference schemes (Park and Petrosian 1996).

Boundary conditions As stated by Park and Petrosian (1996) any boundary condition which is a linear combination of $F$ and $F^{\prime}(p)=\partial_{\mathrm{p}} F$ is viable for Eq. (35) if the points $p_{1}$ and $p_{2}$ at which they are taken fulfill $0<p_{1}<p<p_{2}<\infty$. So we end up with two types of boundary conditions either with no particle $F\left(p_{1}\right)=F\left(p_{2}\right)=0$ or with no flux $\phi\left(p_{1}\right)=\phi\left(p_{2}\right)=0$ at the boundaries, where $\phi(p)=-\left(p^{2} D_{\mathrm{pp}} F+a(p) F\right)$. The choice of one condition with respect to the other depends on the specific problem under investigation. A drawback of the no-particle condition is that it does not respect the particle number conservation.

Numerical schemes A simple way to solve Eq. (35) is to use an explicit finite difference method (FDM) with fluxes evaluated at grid midpoints, namely

$$
\frac{F_{j+1}^{n+1}-F_{j}^{n}}{\Delta t}=-\frac{1}{p_{j}^{2}}\left(\frac{\phi_{j+1 / 2}^{n}-\phi_{j-1 / 2}^{n}}{\Delta p}\right)-\frac{F_{j}^{n}}{\tau_{\mathrm{esc}}\left(p_{j}\right)}+Q^{n}\left(p_{j}\right) .
$$

Time is discretized as $\Delta t=t_{n+1}-t_{n}$ and momentum is discretized following a constant logarithmic mesh where $\Delta p_{\mathrm{j}} / p_{\mathrm{j}}=$ constant. We write $\Delta p_{\mathrm{j}}=\left(p_{\mathrm{j}+1}-p_{\mathrm{j}-1}\right) / 2$. The fluxes are calculated at midpoints defined as $p_{j+1 / 2}=\left(p_{j+1}+p_{j}\right) / 2$. The coefficients entering in the flux calculation are evaluated as, e.g., $a_{j+1 / 2}=\left(a\left(p_{j+1}\right)+a\left(p_{j}\right)\right) / 2$ instead of a direct evaluation at $p_{j+1 / 2}$. For an explicit scheme the CFL condition (see Sect. 5.2.1) $\Delta t / \Delta p_{j}^{2}<p_{j}^{2} / D_{\mathrm{pp}, \mathrm{j}}$ usually produces prohibitively small time steps. 


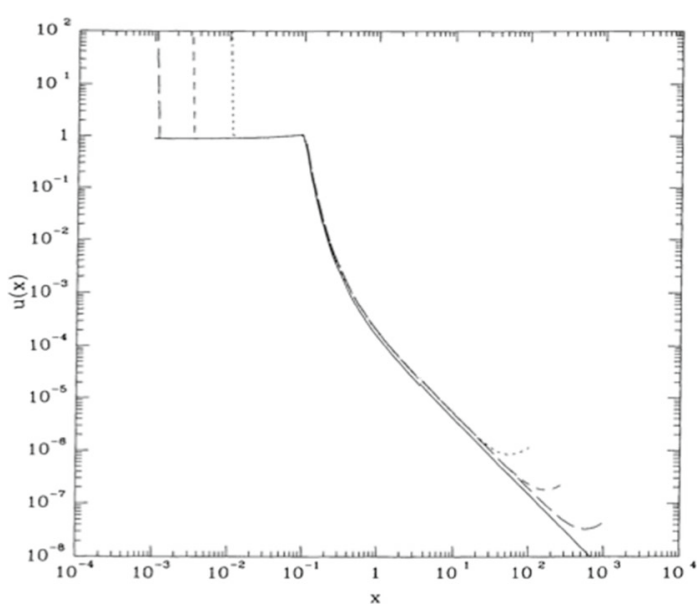

Fig. 20 Time-dependent solutions of the FP problem $\partial_{t} F=\partial_{p}\left(p^{3} \partial_{p} F-p^{2} F\right)-F+\delta\left(p-p_{0}\right) \delta(t)$ over a logarithmically-spaced mesh in momentum. The injection momentum is $p_{0}$. Three different numerical boundaries are compared: $p_{1}=10^{-2}$ and $p_{2}=10^{2}$ (short-dashed lines); $p_{1}=10^{-2.5}$ and $p_{2}=10^{2.5}$ (medium-dashed lines); $p_{1}=10^{-3}$ and $p_{2}=10^{3}$ (long-dashed lines) The steady state numerical solutions were obtained at $t=10$ in normalized units. Image reproduced with permission from Park and Petrosian (1996), copyright by AAS

Semi-implicit and implicit methods can be used to circumvent this problem; they are obtained by changing $n$ to $n+1 / 2$ and $n+1$ in the RHS of Eq. (36) respectively. These methods lead to the derivation of a tridiagonal system of equations that can easily be solved once the boundary conditions are selected. For a given class of method, schemes then differ by the way the flux is calculated. One efficient implicit method is due to Chang and Cooper (1970) and a well-known semi-implicit calculation is the CrankNicholson method (see Press et al. 2002). These methods are second order in time and second order in momentum for a uniform grid and first order in momentum for a nonuniform grid. Park and Petrosian (1996) show that the no-flux condition and the implicit Chang-Cooper scheme ensure positive solutions of 1D FP problems contrary to the Crank-Nicholson method (see an example of a solution of a FP problem in Fig. 20). While accounting for losses in $\phi(p)$ it is useful to adapt the time step to the dominant loss timescale. Donnert and Brunetti (2014) use a time step $\Delta t=1 / 2 \min \left(t_{\text {loss }}\left(p_{j}\right)\right)$, where $t_{\text {loss }}(p)=a(p) / p$.

A note on particle transport and stochastic acceleration in hot plasmas FDM are widely used in CR physics but they are also used to solve radiative transfer problems in hot plasmas which develop in corona or in jets associated with compact objects. The radiative transfer in accretion disk corona can be treated in 1D assuming some particular geometry (usually slab-type or spherical) for the source of high-energy particles which allows to derive an escape probability and hence a simple expression for $\tau_{\text {esc }}$ in Eq. (35). In this approach the coupled system of electron-positron plasma and its associated photon field can be described by a set of FPEs. However, a major 
difficulty to simulate such plasma systems is that they involve non-local processes in momentum. ${ }^{38}$ This is for instance the case for the Compton scattering process (Nayakshin and Melia 1998). In that case, the fluxes are expressed in terms of integrals over lepton and photon populations (Belmont et al. 2008; Vurm and Poutanen 2009; Marcowith et al. 2013). ${ }^{39}$ Aside from Compton scattering, integrals also result from the calculation of other processes: pair production and annihilation, Coulomb losses, synchrotron losses. These codes solve the diffusion problem usually using ChangCooper-type methods. However, as noticed by Belmont et al. (2008), the radiative transfer problem requires a high accuracy in momentum to preserve a high level of particle number and energy conservation. The Chang-Cooper method which is only first order accurate on non-uniform grid needs to be modified using both grid center and faces and calculating the momentum derivatives as $\partial_{\mathrm{p}} F=\left(F_{\mathrm{j}+1 / 2}-F_{\mathrm{j}-1 / 2}\right) / \Delta x_{\mathrm{j}}$ and $\partial_{\mathrm{p}}^{2} F=\left(F_{\mathrm{j}+1}-F_{\mathrm{j}}\right) / \Delta p_{\mathrm{j}+1 / 2}-\left(F_{\mathrm{j}}-F_{\mathrm{j}-1}\right) / \Delta p_{\mathrm{j}-1 / 2}$. The integral parts have to be calculated using specific treatments. First, the different elements of the cross sections are stored and then interpolated during the course of the runs. Then, boundary conditions are different for the FP and the transfer parts. The transfer part has a walltype boundary condition which includes a modification of the differential cross section [see Belmont et al. (2008) for details]. Finally, integrals used to calculate the Compton process can be treated differently depending on the photon energy with respect to the electron energy from a continuous process at low energy leading to a derivative term and to a full integral calculation in the Klein-Nishina limit. Compton scattering of photons involves the same kind of treatment and is applied depending on the electron energy [see (Vurm and Poutanen 2009) for details].

Multi-variable FPE Astrophysical or space plasmas usually involve multi-dimensional diffusion-advection processes. The study of CR propagation in the Milky Way requires to account for several complex effects: CR spallation reactions, radioactive decay, anisotropic diffusion with respect to the background magnetic field direction, etc. Specific numerical tools have been developed to handle this complexity. ${ }^{40}$ Most of CR transport codes use multi-dimensional finite difference methods, this is the case for GALPROP and DRAGON which adopt a Crank-Nicholson method. In multi-dimensional problems this method leads to a non-tridiagonal system of equations to solve. It is solved usually adopting an iterative procedure like the Gauss-Seidel relaxation method (Press et al. 2002). The integration is adapted to the specific diffusion problem by starting from a large time step and reducing it as the stationary solution is reached (Strong and Moskalenko 1998). Both GALPROP and DRAGON codes also use an operator splitting technique to handle multi-dimensional diffusion problems (Press et al. 2002). The technique of operator splitting consists in splitting the time integration in Eq. (35) or

\footnotetext{
38 Some processes are also in principle non-local in space, this is the case for instance of the inhomogeneous synchro-Compton effect in jets, i.e., the Inverse Compton scattering of low energy photons generated by synchrotron radiation by a population of relativistic electrons (see Ghisellini et al. 1985).

39 A version of such radiative transfer codes adapted to GRBs can be found in Vurm et al. (2011).

40 We point towards the corresponding code websites: DRAGON: https:/github.com/cosmicrays, GALPROP: https://galprop.stanford.edu/code.php, PICARD: http://astro-staff.uibk.ac.at/ kissmrbu/Picard.html Let us also mention the semi-analytical tool USINE, https://dmaurin.gitlab.io/USINE/, where the FPE is solved using a path integral method.
} 
its multi-dimensional generalization into a succession of simpler operations involving $\mathrm{N}$ different operators $L_{\mathrm{i}}$, such that

$$
\partial_{t} F(p, t)=\sum_{i=1}^{N} L_{\mathrm{i}} F(p, t) .
$$

Each operator contributes to move the solution from $F^{n}$ to $F^{n+1}$ as $F^{n+1}=$ $\prod_{i=1}^{N} \mathcal{L}_{\mathrm{i}} F^{n}$ where each finite difference operator solves a part of the numerical problem. One difficulty with this method is that operator actions do not commute, hence one usually has to proceed with trials with a guess of the correct solution to select the correct operator ordering. The DRAGON code designed in cylindrical coordinates uses a series of operators associated to each relevant transport process. For instance the operator associated with diffusion along galactic vertical height $z$ is $L_{\mathrm{z}}=D_{\mathrm{zz}} \partial_{\mathrm{z}}^{2} F(z, r, p, t)+\partial_{\mathrm{z}} D_{\mathrm{zz}} \partial_{\mathrm{z}} F(z, r, p, t)$. Similarly other operators are derived for diffusion along other space variable $r$ (the galactic radius), momentum loss or advection (Evoli et al. 2017). Then each derivative is treated using a Crank-Nicholson scheme. The PICARD code uses a different numerical approach as it first solves a stationary problem and also as the momentum evolution is treated using an integration instead of a FDM (Kissmann 2014).

Multi-dimensional numerical solutions of FP problems is an active research field with a rich variety of solvers based on three main approaches: finite difference methods as we just discussed (FDM), finite element methods (FEM) or path integrals techniques. For most of them they still wait to be applied in the context of astrophysical or space plasma research.

\subsubsection{Stochastic differential equations}

Stochastic differential equations or SDEs are a very efficient way to solve complex multi-dimensional Fokker-Planck problems with simple numerical schemes, although SDE schemes can become themselves rather complex. We invite the interested reader to consult some monographs cited in Strauss and Effenberger (2017). These authors provide an overview of the use of SDE in the fields of DSA, CR transport in the ISM and space plasmas. The interested reader can consult this complete review to what concerns space plasmas problems. Below we bring a complementary discussion on the use of SDE in the context of shock acceleration. The intrinsic idea behind SDE is to derive a set of equations of motion which reproduce the random walk in each of the stochastic variables which describe the phase space evolution of a particle. Kruells and Achterberg (1994) demonstrate the equivalence between a FPE and a set of SDEs. The simplest SDE scheme is the Ito first order explicit scheme. As an example let us write the SDE for a $1 \mathrm{D}$ random walk in a space $\mathrm{x}$ direction of a particle represented by a diffusion coefficient $D(x, t)$. Let us assume also that the particle is advected with a speed $u(x, t)$. The first order forward explicit Ito scheme then writes the increment of the position of the particle within a time step $\Delta t$ as 


$$
\Delta x=\left(u(x, t)+\frac{\partial D(x, t)}{\partial x}\right) \Delta t+\xi_{\mathrm{x}} \sqrt{2 D(x, t) \Delta t}=\Delta x_{\mathrm{adv}}+\xi_{\mathrm{x}} \Delta x_{\mathrm{diff}} .
$$

We note $V(x, t)=u(x, t)+\partial D(x, t) / \partial x$. This equation shows that the particle path has two terms, the first term is deterministic and reproduces a forward Euler increment due to advection. The second term is stochastic and describes a diffusion as $\Delta x \propto$ $(\Delta t)^{1 / 2}$. The term $\xi_{\mathrm{x}}$ is a random variable usually sampled over a Gaussian distribution with 0 mean and variance 1 . The particle distribution can then be reconstructed using a large number of particles. The method is simple; however, it can suffer from noise in parts of the phase space sampled by only a few particles. The latter issue can be partly handled using a particle-splitting scheme (Yamazaki et al. 2015) where the weight attributed to a particle is split over several particles when reaching a region of the phase space with low statistics, as can be the case in the energy space if the particle distribution has an exponential cut-off.

More generally, the diffusive term in Eq. (38) is a Wiener processes $W(t, x)$ which models the Brownian motion of a particle in an homogeneous medium, we write $d W(x, t) / d t=\xi_{\mathrm{x}}$. More complex SDE schemes can be interesting to use if necessary, like schemes backward in time in order to start from a known distribution and reconstruct the particle injection at sources. This way has the advantage to improve statistics if we want to have information at a particular location, corresponding for instance to a satellite. Higher order schemes in space and time are possible by doing a Taylor expansion of both advection and stochastic parts of Eq. (38). Schemes stable in time can be obtained by searching advection and diffusive terms at a time $t^{\prime}=t+\theta \Delta t$, where $\theta$ is to be taken between 0 and 1 (Smith and Gardiner 1989).

DSA with SDEs The study of shock acceleration using SDEs requires some care in fixing the time step $\Delta t$ (Kruells and Achterberg 1994). In shock acceleration studies the shock front is usually obtained from a fluid code, so has a finite width $\Delta x_{\mathrm{sh}}$ traced by a few grid cells. The condition over the time step to describe the DSA process properly is then $\Delta X_{\text {adv }}<\Delta x_{\text {sh }}<\Delta X_{\text {diff }}$. The first inequality allows particles to stay around the shock to get accelerated whereas the second inequality allows the particle to sample the up- and downstream media correctly. However, if the diffusion coefficient is an increasing function of the particle energy, i.e., $\partial D(x, E, t) / \partial E>0$ it is possible to find a threshold energy $E^{*}$ for which the condition $\Delta x_{\mathrm{sh}}=\Delta X_{\mathrm{diff}}\left(E^{*}\right)$ is fulfilled (Casse and Marcowith 2005; Schure et al. 2010). Below $E^{*}$ the shock acceleration process can not be properly treated. One possibility to address this problem is to sharpen artificially the shock (Casse and Marcowith 2003). This method can be easily handled in 1D (Marcowith and Casse 2010) but is difficult to construct in 2 or 3D as the shock front starts to corrugate. Another difficulty is that at an non-parallel shock, the MHD Rankine-Hugoniot conditions induce a discontinuous diffusion coefficient up- and downstream. Quite generally the diffusion transition at the shock can be decomposed into a continuous component $D_{\mathrm{c}}$ and a jump at the shock front $\Delta D=D_{\mathrm{u}}-D_{\mathrm{d}}$ expressed in terms of the up- and downstream diffusion coefficients. The diffusion coefficient can then be written as

$$
D(x)=D_{\mathrm{c}}(x)+\Delta D \delta\left(x-x_{\mathrm{sh}}\right),
$$


where $x_{\text {sh }}$ is the shock position (Marcowith and Casse 2010). Zhang (2000) proposes to account for the discontinuous part using a skewed Brownian motion which introduces an asymmetric shock crossing probability. To proceed we introduce a new variable $\tilde{x}=x \zeta(x)$ where

$$
\zeta(x)= \begin{cases}\epsilon & \mathrm{x}<\mathrm{x}_{\mathrm{sh}} \\ 1 / 2 & \mathrm{x}=\mathrm{x}_{\mathrm{sh}} \\ (1-\epsilon) & \mathrm{x}>\mathrm{x}_{\mathrm{sh}}\end{cases}
$$

with $\epsilon=D_{\mathrm{u}}\left(x_{\mathrm{sh}}\right) /\left(D_{\mathrm{d}}\left(x_{\mathrm{sh}}\right)+D_{\mathrm{u}}\left(x_{\mathrm{sh}}\right)\right)$. Achterberg and Schure (2011) propose a more general scheme adapted to shock configuration with strong gradients in the diffusion coefficient. This situation occurs especially upstream, in the shock precursor, in case of strong magnetic field amplification. The scheme involves a second-order accuracy predictor-corrector method [see Section 4 in Achterberg and Schure (2011) for details]. The scheme is however much slower than the simple Ito scheme and it is necessary to switch from one scheme to the other in order to save simulation resources.

One also has to account for the particle increment in energy or momentum at each shock crossing. It is also possible to use an explicit Ito scheme for CR energy or momentum similarly to Eq. (38). If stochastic acceleration can be neglected, Marcowith and Kirk (1999) introduce an implicit scheme:

$$
\Delta \ln (p)=-\left(a_{\operatorname{loss}} p+\frac{1}{3} \frac{d u}{d x}\right) \Delta t
$$

where $a_{\text {loss }}$ is a loss rate and the second term accounts for the increase in particle momentum from shock acceleration. The implicit scheme rewrites the particle position with time as a linear interpolation $x=(\Delta x / \Delta t) t$. Eq. (41) has the solution

$$
\ln \left(p\left(t^{\prime}\right) / p(t)\right)=-\ln \left(F_{I}+L_{\mathrm{s}}\right)
$$

where $F_{I}=\exp ((\Delta V / 3) \Delta t / \Delta x)$ gives the momentum increment by DSA and

$$
L_{\mathrm{s}}=a_{\mathrm{s}} \frac{\Delta t}{\Delta x} p \int_{x(t)}^{x\left(t^{\prime}\right)} \exp \left(\frac{\Delta V}{3} \frac{\Delta t}{\Delta x}\right) d x^{\prime},
$$

where $L_{\mathrm{S}}$ accounts for the effect of losses. The increment $\Delta x$ is calculated from the $\mathrm{SDE}$ in $\mathrm{x}$, which is evaluated at $t^{\prime}=t+\Delta t$. The new momentum is obtained from Eq. (42) calculated using $x\left(t^{\prime}\right)=x(t+\Delta t)$. Figure 21 gives the shock solution for electrons including synchrotron losses.

The SDE method has proven to be very efficient in calculating particle acceleration by DSA at non-relativistic shocks in $1 \mathrm{D}$ and even in 2.5D in the context of jets (Casse and Marcowith 2003, 2005). The method can in principle be coupled to MHD solutions by sub-cycling the MHD timestep (see Sect. 5.5). To our knowledge no scheme has yet included CR back-reaction over the thermal plasma, but solutions proposed by other Monte-Carlo models (see Sect. 3.6.3) should be applicable to this particular technique. 


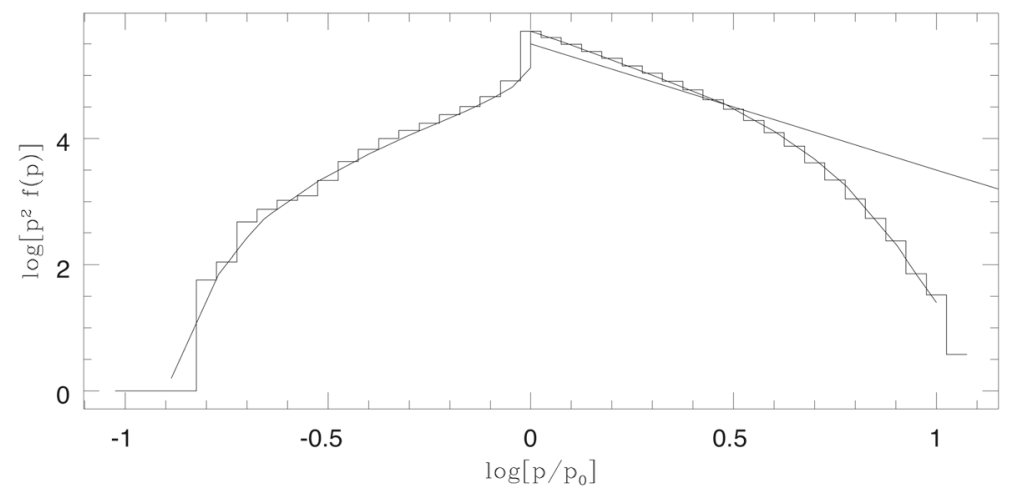

Fig. 21 Shock electron distribution including synchrotron losses. Particles are injected at momentum $p_{0}$ for $a_{\mathrm{s}, \mathrm{u}}=10, a_{\mathrm{s}, \mathrm{d}}=1$ and a compression ratio $r=4$ compared to the analytical solution of Webb et al. (1984). The solid line shows a solution $f(p) \propto p^{-4}$. Image reproduced with permission from Marcowith and Kirk (1999), copyright by ESO

Relativistic shocks and SDEs In the context of relativistic shock two difficulties emerge if one wants to apply the SDE method to the problem of particle acceleration because the shock is moving almost as fast as the particles. First, a simple scheme as in Eq. (38) may lead to a violation of the causality principle, because over the diffusive step the particle speed $\Delta x / \Delta t$ can exceed the speed of light. Second, DSA is based on the diffusive approximation which requires the ratio of the particle speed to the shock speed to be small. Achterberg et al. (2001) evaluate particle acceleration in the shock rest frame but simulate the spatial diffusion process from the pitch-angle scattering process and hence reconstruct particle trajectories ([see also Bednarz and Ostrowski 1998)]. These works retrieve the shock particle distribution produced by scattering by an isotropic turbulence $f(p) \propto p^{-4.2}$ (see Sect. 2.4).

\subsubsection{Simulating shock acceleration using a Monte-Carlo method}

Ellison and Eichler (1984), Jones and Ellison (1991) proposed a Monte-Carlo method to simulate particle pitch-angle scattering around non-relativistic shocks. The particle mean free path is assumed to scale as a function of the particle rigidity as $\lambda \propto R^{a} / \rho$, where $R=p c / q$ is the particle rigidity and $\rho$ is the fluid mass density. The momentum vector follows a random walk which produces a variation of the particle pitch-angle $\delta \alpha$. The scattering is assumed to be elastic and isotropic in the fluid rest frame. The particle are injected upstream from the thermal plasma. The CR pressure is reconstructed at different distances from the shock front; particle-splitting technique is used in order to improve statistics at high energies. The CR pressure term is then included in the Rankine-Hugoniot conditions to account for CR backreaction over the shock solutions. Finally, a far escape boundary is adopted to calculate the escaping energy flux carried by the particles. An example of results can be seen in Fig. 43, compared with two other methods discussed elsewhere in this review. 
Recently the technique has been used to study the effect of magnetic field amplification in NLDSA at non-relativistic SNR shocks (Vladimirov et al. 2006), as well as acceleration at relativistic shocks in GRB afterglows (Warren et al. 2015).

\section{Small and meso-scale numerical particle acceleration studies}

The understanding of the initial stages of particle acceleration in astrophysical plasma relies on the non-linear interplay between the particle distribution function and electromagnetic fields. The inherent non-linearity of the process prevents the development of robust analytic models, unless numerical simulations provide basic guidelines of the behavior of the system. This is especially true when particles produce the turbulence responsible for their self-confinement and acceleration around the shock front. The improvement in the computation power during last decades allowed for significant progresses in this field of research using computationally expensive, yet considered as $a b$-initio, PIC simulations. Despite the fact that the astrophysical sources space and time scales are out of reach of PIC simulations, a large number of fundamental questions have been addressed and, sometimes, answers provided using this technique. In this section, we review a number of works that investigate the question of particle acceleration efficiency at shocks (Sect. 4.1) and in magnetic reconnection (Sect. 4.2) processes, using PIC simulations. Before we further proceed, we first introduce some vocabulary concerning the different category of shocks investigated with the help of PIC simulations.

Collisionless shocks classes Collisionless shocks are mediated by collective plasma effects (Sagdeev 1966). In this sense, more refined classification is required then for hydrodynamical/MHD shocks (weak, strong, radiative, fast, slow, parallel, oblique, perpendicular) where the shock is expected to be mediated by binary collisions between particles. Seminal (Sagdeev 1966) as well as recent studies (e.g., Stockem et al. 2014b, a; Bret et al. 2014; Ruyer et al. 2016) considered and demonstrated the dominant role of small scale plasma instabilities in forming and mediating collisionless shocks. Different types of instabilities are dominant depending of plasma beta, composition, shock Mach number and upstream magnetic field orientation with respect to the shock propagation direction. This translates into a bestiary of different plasma instabilities at play when describing the shock structure. Commonly, the separation into electrostatic, Weibel-mediated and magnetised shocks is done. Non-relativistic, weakly magnetised and low-Mach number shocks are believed to be mediated by electrostatic effects (two-stream, Buneman instabilities). With increasing Mach number $\left(M_{a} \gg 1\right)$ or going into relativistic regime, weakly magnetised shocks are mediated by Weibel-filamentation (Bret et al. 2014; Huntington et al. 2015). Strongly magnetised shocks are typically mediated by coherent magnetic reflection of particles on the shock barrier. In this case the shock width is of the order of ion gyroradius.

\subsection{Shock acceleration numerical experiments: PIC simulations}

The short chronological version is the following. In the pioneering studies 1D3V geometry was adopted because of numerical cost (e.g., Biskamp and Welter 1972). Several 
physical processes were evidenced in this way, such as shock front self-reformation (e.g., Lembege and Dawson 1987; Lembege and Savoini 1992) or positron acceleration through resonant absorption of ion-cyclotron waves (Hoshino et al. 1992). However, this configuration was found to be too restrictive to trigger the Fermi process in most of cases, especially for quasi-perpendicular shocks. Next, multidimensional simulations were long enough to form the shock, but no evidence of first-order Fermi acceleration was found (Frederiksen et al. 2004; Hededal and Nishikawa 2005; Kato 2007; Dieckmann et al. 2008). This raised the question whether shocks can accelerate particles self-consistently or some external source of turbulence was necessary. More recent studies were able to form the shock and follow its propagation long enough to allow several Fermi cycles and produce extended power-law particle distribution self-consistently (e.g., Spitkovsky 2008b; Martins et al. 2009; Sironi and Spitkovsky 2011; Plotnikov et al. 2018; Crumley et al. 2019; Lemoine et al. 2019b). Yet, even in the longest simulations the power-law spans no more than two orders of magnitude in particle energy, reflecting the challenging nature for fully kinetic simulations to reach astrophysical space and timescales.

\subsubsection{Ultra-relativistic shocks}

The ultra-relativistic regime is particularly interesting for several reasons. ${ }^{41}$ (i) Energy gain per cycle is large, $\Delta E / E \simeq 2$ instead of $\Delta E / E=\beta_{\text {sh }} \ll 1$ in the non-relativistic regime and (ii) Scattering time must be short, otherwise particles get advected within the downstream flow as the shock front recedes rapidly (the shock front moves away with velocity equal $c / 3$ in the frame where the downstream plasma is at rest). These two reasons mean that the build-up of the non-thermal power law is faster in the ultrarelativistic regime than in the non-relativistic case. Hence, one can diagnose whether relativistic shock accelerate particles efficiently or not for a given parameter regime on a timescale of several $10^{3} \omega_{\mathrm{pe}}^{-1}$ (given upstream flow magnetization $\sigma$, magnetic field inclination with respect to the shock propagation direction and plasma composition). On the other hand, fast and efficient particle acceleration prompted early Monte-Carlo and semi-analytical studies to suggest that relativistic shocks are viable candidates for the acceleration of Ultra High Energy CRs (UHECRs).

For the reasons outlined just before, the demonstration of the first order Fermi operability in PIC simulations was firstly done in the regime of relativistic shocks by Spitkovsky (2008b). In the non-relativistic case it was done several years later, as it requires much longer simulation time (e.g., Kato 2015; Park et al. 2015).

We now turn to the discussion of different studies that used PIC simulations to understand the physics of ultra-relativistic shocks. The studies go from $1 \mathrm{D}$ to 3D, deal with different plasma compositions, different magnetic field geometries and magnetization. Table 1 provides a (non-exhaustive) list of such studies and presents some relevant numerical parameters that they used. Below we discuss different type of micro-instabilities which are reviewed in Marcowith et al. (2016), Bret (2009).

\footnotetext{
${ }_{41}$ See also the recent review article by Vanthieghem et al. (2020) on the physics of weakly magnetized shocks.
} 
Table 1 Table of different PIC studies of relativistic shocks, referenced by the authors names and publication dates

\begin{tabular}{llllll}
\hline References & Compos. & Dim. & $m_{\mathrm{i}} / m_{\mathrm{e}}$ & $\sigma$ & $\theta_{\mathrm{B}}^{\circ}$ \\
\hline Langdon et al. (1988) & $e^{-}-e^{+}$ & $1 \mathrm{D}$ & 1 & {$[0.1 ; 13.3]$} & 90 \\
Gallant et al. (1992) & $e^{-}-e^{+}$ & $1 \mathrm{D}$ & 1 & {$\left[3 \cdot 10^{-5} ; 5\right]$} & 90 \\
Hoshino et al. (1992) & $e^{-}-e^{+}-i$ & $1 \mathrm{D}$ & 20 & {$\left[5 \cdot 10^{-3} ; 0.5\right]$} & 90 \\
Nishikawa et al. (2003) & $e^{-}-i$ & 3D & 20 & 0 & - \\
Frederiksen et al. (2004) & $e^{-}-i$ & 3D & 16 & 0 & - \\
Hededal et al. (2004) & $e^{-}-i$ & 3D & 16 & 0 & - \\
Spitkovsky (2005) & $e^{-}-e^{+}$ & 3D & 1 & {$[0 ; 0.1]$} & 90 \\
Lyubarsky (2006) & $e^{-}-i$ & 1D & 50 & $3 \cdot 10^{-3}$ & 90 \\
Kato (2007) & $e^{-}-e^{+}$ & 2D & 1 & 0 & - \\
Hoshino (2008) & $e^{-}-i$ & 1D & 50 & $2 \cdot 10^{-3}$ & 90 \\
Dieckmann et al. (2008) & $e^{-}-i$ & 1D,2D & 400 & $2.5 \cdot 10^{-3}$ & $\simeq 10$ \\
Chang et al. (2008) & $e^{-}-e^{+}$ & 2D & 1 & 0 & - \\
Spitkovsky (2008a) & $e^{-}-e^{+}$ & 2D & 1 & 0 & - \\
Spitkovsky (2008b) & $e^{-}-i$ & 2D & $\leq 10^{3}$ & 0 & - \\
Keshet et al. (2009) & $e^{-}-e^{+}$ & 2D & 1 & 0 & - \\
Martins et al. (2009) & $e^{-}-i$ & 2D & 32 & 0 & - \\
Sironi and Spitkovsky (2009) & $e^{-}-e^{+}$ & 2D & 1 & 0.1 & {$[0 ; 90]$} \\
Sironi and Spitkovsky (2011) & $e^{-}-i$ & 2D,3D & $\mathbf{1 6}$ & {$\left[10^{-5} ; 0.1\right]$} & {$[0 ; 90]$} \\
Haugbølle (2011) & $e^{-}-i$ & 2D,3D & 16 & 0 & - \\
Sironi et al. (2013) & $e^{-}-e^{+} / i$ & 2D,3D & $1 \& \mathbf{2 5}$ & {$[0 ; 0.1]$} & 90 \\
Bret et al. (2013) & $e^{-}-e^{+}$ & 2D & 1 & 0 & - \\
Ardaneh et al. (2015) & $e^{-}-i$ & 3D & 16 & 0 & - \\
Plotnikov et al. (2018) & $e^{-}-e^{+}$ & 2D & 1 & {$[0 ; 5]$} & 90 \\
Crumley et al. (2019) & $e^{-}-i$ & 2D & $\mathbf{6 4}$ & 0.007 & 10 55 \\
\hline & & & 16 &
\end{tabular}

Mass ratio values indicated with bold font indicate that larger values were also explored

1D studies The first exploration of relativistic shocks was motivated by the study of the termination shock physics in Pulsar Wind Nebulae. Langdon et al. (1988) performed 1D PIC simulations of perpendicular magnetized shocks in pair plasma with upstream Lorentz factors $\gamma_{0}=20$ and 40 and Alfvénic Mach numbers going from 24 to 154. The size of the simulation box was about 10 Larmor radii for $\sigma=0.1$ and about 100 Larmor radii for $\sigma=13.3$. The shock front formed by magnetic reflection between the incoming and wall-reflected plasma. Well-formed shocks exhibited a soliton-like structure where most of dissipation occurs through maser synchrotron instability. Strong electromagnetic precursor emission was observed in these shocks but no particle acceleration. More systematic study was performed by Gallant et al. (1992) where electromagnetic precursor energy was systematically derived. The most efficient precursor emission was observed for $\sigma \sim 0.1$ where it carries about $10 \%$ of the incoming kinetic energy. Based on these results, the authors concluded that 
magnetized perpendicular relativistic shocks in pair plasma are not efficient particle accelerators. An interesting particle acceleration mechanism was observed by Hoshino et al. (1992) in the case where a small fraction of the plasma are ions (electron-positronion composition). In this case the shock structure is modified by ions, even if their number fraction is small compared to positrons. Magnetosonic waves emitted by ions are resonantly absorbed by upstream positrons that produces a non-thermal tail in positron distribution function. Electrons and ions distributions are still Maxwellian.

Concerning the studies of shocks in electron-ion plasma using 1D simulations, Lyubarsky (2006) and Hoshino (2008) explored mildly magnetized regimes. Lyubarsky explored the effect of the electromagnetic precursor on the incoming electrons and found that the relativistic oscillation of electrons in the field of the wave results in temperature equipartition between electrons and ions, once they reach downstream medium. The theoretical model was confirmed by a 1D PIC simulation with the mass fraction $m_{i} / m_{e}=200$, upstream Lorentz factor $\gamma_{0}=50$, upstream magnetization $\sigma=0.003$ and 0.6. No supra-thermal tail in electron distribution was found. This finding was contrasted by the study of Hoshino (2008) where it was found that the precursor wave produces a non-thermal tail in ion and electron distribution functions. The mechanism for particle acceleration is expected to be of wakefield nature.

Early multi-dimensional studies The opening of additional degrees of freedom in directions transverse to the shock propagation is essential for relativistic shock physics. For instance, the dominant instability in low- $\sigma$ regime, Weibel-filamentation, is artificially suppressed in 1D because it is only triggered by a non-zero $k_{\perp}$, where $k_{\perp}$ is the transverse wavenumber to the beam propagation direction. This regime is particularly relevant for astrophysics (for instance in the case of the GRB or AGN studies of the propagation of the forward shock) because the magnetization in the ISM is $\sigma_{\text {ISM }} \sim 510^{-11} B_{\mu \mathrm{G}}^{2} / n_{\mathrm{cm}^{-3}}$, where the magnetic field strength is in units of $\mu$ Gauss and the ambient density in units of $\mathrm{cm}^{-3}$. Consequently, the observation of filamentation and concomitant trigger of the Fermi process relies on multi-dimensional configuration of the simulation. This restriction is less severe in non-relativistic case for quasi-parallel shocks, where non-resonant streaming or Bell instability can be triggered in 1D and sustains particle scattering (or mirroring) on both sides of the shock front.

Additional step from 2D to 3D is important to correctly deal with particle scattering properties as the topology of the turbulent magnetic field is different.

The early multi-dimensional exploration of unmagnetized relativistic shocks was done with full 3D3V but short simulations. Nishikawa et al. (2003) simulated a relativistic jet with $\Gamma_{\text {jet }}=5$ propagating into an unmagnetized electron-ion plasma at rest. These simulations were done using TRISTAN code (Buneman 1993). The simulation box was small $[15 \times 8 \times 8 \times 15]\left(c / \omega_{\text {pe }}\right)^{3}$ but still large enough to capture the electron-scale Weibel-filamentation instability and the simulation time was $T_{\mathrm{sim}}=23.4 \omega_{\mathrm{pe}}^{-1}=5.23 \omega_{\mathrm{pi}}^{-1}$. Capturing only the initial stage (shock not formed) the authors still demonstrated the importance of the Weibel-filamentation instability. Frederiksen et al. (2004) performed 3D simulation of unmagnetized colliding plasma clouds with density ratio $n_{i n j} / n_{0}=3$, relative Lorentz factor $\Gamma_{\text {jet }}=3$ and ion to 
electron mass ratio $m_{i} / m_{e}=16$. The simulation box was larger $L_{x} \times L_{y} \times L_{z}=$ $[200 \times 200 \times 800]\left(c / \omega_{\mathrm{pe}}\right)^{3}=[10 \times 10 \times 40]\left(c / \omega_{\mathrm{pi}}\right)^{3}$ and the simulation time longer $T_{\mathrm{sim}}=480 \omega_{\mathrm{pe}}^{-1}=120 \omega_{\mathrm{pi}}^{-1}$. The main result of this work is that the initial filamentation grows from electron to ion scales. However, the simulation was just long enough to reach the ionic scale, the saturation was just reached and the shock was not completely formed. Hededal and Nishikawa (2005) continue in the same direction by producing 3D simulation with similar parameters but longer time $T_{\mathrm{sim}}=360 \omega_{\mathrm{pi}}^{-1}$. The authors observed an interesting electron acceleration mechanism when electrons cross the ionic current channels. This produced a non-thermal tail in the electron distribution function $d N / d E \propto E^{-2.7}$. However, here again the shock was not fully formed because downstream ion distribution was still far from isotropy. The same electron energization mechanism was later found by Ardaneh et al. (2015), who studied the jet-ambient medium interaction by means of 3D simulations. These authors suggested that electrons can also be pre-accelerated by SSA mechanism during the shock formation.

Spitkovsky (2005) considered a pure $e^{-}-e^{+}$plasma where there is no scale separation. In this way the typical shock formation and evolution time is much shorter than for electron-ion plasma. Different magnetizations were explored $\sigma \in[0,0.1]$ with the relative Lorentz factor $\gamma_{0}=15$, in 3D3V configuration. The shock was triggered by reflection of incoming flow on a conducting wall. In this way, the interaction of wall-reflected and incoming flows produces the shock [simulation frame $=$ downstream rest frame]. The box size was similar to previous studies $[200 \times 40 \times 40]\left(c / \omega_{\text {pe }}\right)^{3}$. The shock was formed as the downstream plasma reached the expected RankineHugoniot jump conditions and the distribution function isotropized in the overlap region. As previously, for the unmagnetized case $\sigma=0$ the shock is mediated by Weibel-filamentation but strongly magnetized shock $\sigma=0.1$ has a very different structure shaped by the perturbation of the upstream magnetic field: the incoming flow is coherently reflected on the magnetic barrier at the shock front position. The shock is then mediated by magnetic reflection. In all cases the author did not find evidence of non-thermal part in particle distribution that suggested that the acceleration is either slow to setup or not present at all. Very similar conclusions were found by Kato (2007) where 2D simulations of a shock with $\gamma_{0}=2.24$ in pair plasma were performed. This study demonstrated that the small scale magnetic field fluctuations, self consistently produced by Weibel-filamentation, is able to mediate unmagnetized collisionless shocks. The ratio of magnetic energy to the incoming kinetic energy of the upstream flow, $\xi_{\mathrm{B}}=\delta B^{2} /\left(4 \pi \gamma_{\mathrm{sh}}^{2} \rho c^{2}\right)$, peaks at the shock front $\left(\xi_{\mathrm{B}}=0.14\right)$ where the incoming mono-directional flow is isotropized and rapidly decreases downstream by phase-mixing.

Recent multi-dimensional studies The main difference with the early studies is (i) full formation of pair and electron-ion shocks and (ii) the realization of efficient particle acceleration through first-order Fermi process in a self-consistent way by following the evolution of shocks on longer timescale.

Chang et al. (2008) addressed the question of the fate of the magnetic turbulence downstream of relativistic unmagnetized shock in pair plasma. The authors used the 
same code as in Spitkovsky (2005) (TRISTAN-MP) with relatively long simulation time $T_{\text {sim }}=3500 \omega_{\mathrm{p}}^{-1}$, where $\omega_{\mathrm{p}}$ is the total plasma frequency. As expected, the unmagnetized shock is mediated by Weibel-filamentation in the precursor. The filamentary magnetic field in the precursor becomes almost isotropic in the downstream medium once the filaments break at the shock front. The coherence scale of the field just behind the front is small $\ell_{c} \sim 10 c / \omega_{\mathrm{p}}$ but grows with increasing distance from the shock front downstream. At the same time magnetic field intensity is found to decrease rapidly. The authors compared the simulations with an analytic model where magnetic field decreases by linear response of the plasma. The intensity is predicted to decrease as $\xi_{B} \propto \delta B^{2} / 8 \pi \propto\left(x_{\text {front }}-x\right)^{-q}$, with $q=2 / 3$. The simulations suggested however that $q=1$ close to the front located at $x_{\text {front }}$ and becomes closer to $2 / 3$ far from the shock front. Due to numerical noise in PIC simulations at finite time of the simulations it is still not clear whether the field strength drops to 0 far from the shock, on macroscopic scales.

The work of Spitkovsky (2008b) presented the first self-consistent demonstration of first-order Fermi process in shocks. The same code as in Spitkovsky (2005) was used, but in 2D configuration for an unmagnetized pair plasma with upstream Lorentz factor of $\gamma_{0}=15$. The simulation time extended up to $T_{\max }=10^{4} \omega_{\mathrm{p}}^{-1}$ and the box size was $\left[10^{4} \times 400\right]\left(c / \omega_{\mathrm{p}}\right)^{2}$. In the intermediate times the shock structure is identical to Kato (2007) and Chang et al. (2008). At late time, a supplementary population of particles builds up as a small fraction of particles in the bulk downstream plasma is able to scatter back into upstream and participate in a standard DSA (in the relativistic regime). The energy gain per cycle is consistent with the analytic prediction $\Delta E / E \simeq 1$. The nonthermal population carries typically $1 \%$ by number and $10 \%$ energy fraction of the total incoming plasma (i.e., the ratio of non-thermal electron energy to the total is $\left.\varepsilon_{\mathrm{e}} \sim 0.1\right)$.

Spitkovsky (2008a) presented the first study of electron-ion relativistic shocks where the shock is fully formed. Several mass ratios were explored $m_{i} / m_{e}=$ $[16,30,100,500,1000]$ and the upstream plasma was unmagnetized. The most important result of this study is that electrons are brought to sub-equipartition with ions during their crossing of the precursor where they are substantially heated inside the ionic filamentary structures. In the downstream medium one gets $T_{e} \simeq T_{i}=$ $\left(\gamma_{0} / 3\right) m_{p} c^{2}$. This result implies an empiric similarity between shocks in pair plasma and in electron-ion plasma because the relativistic mass of particles in the downstream medium is equal. The simulations where still too short to observe the formation of a non-thermal tail in particle distributions.

Keshet et al. (2009) addressed the long term evolution of $\sigma=0$ shocks in pair plasma by performing the longest possible simulations allowed by numerical stability. The simulation box was $[63000 \times 1024]\left(c / \omega_{\text {pe }}\right)^{2}$ and the simulation time was $T_{\text {sim }}=$ $12,600 \omega_{\mathrm{pe}}^{-1}$. They demonstrated that, as particles accelerate to larger energies with time, the precursor size increases and the width of the zone filled with magnetic turbulence increases both upstream and downstream. No convergence was reached, which leaves the question of long-term evolution open.

Martins et al. (2009), by means of 2D PIC simulations with the OSIRIS code, demonstrated that DSA works in electron-ion unmagnetized plasma. The mechanism is very 
similar to the pair plasma case since electrons are at sub-equipartition with ions in the downstream medium.

The influence of magnetic field orientation with respect to the shock normal in strongly magnetized relativistic shocks ( $\sigma=0.1$ and $\left.\gamma_{0}=15\right)$ was studied by Sironi and Spitkovsky (2009) in pair plasma and by Sironi and Spitkovsky (2011) in electronion plasma, using 2D and 3D PIC simulations. These works provide a first survey of parameter space and show in which conditions relativistic shocks are efficient accelerators or not. Even if relativistic shocks are known to be generically quasiperpendicular, the magnetic field inclination parameter, $\theta_{B}$, is important for the shock physics. The very special case of quasi-parallel (or subluminal) shocks, even if very rare, is interesting as it shows very different behavior. In all cases, simulations were carried out for long enough time to form the shock and see whether particle acceleration is present or not. For strongly magnetized shocks $(\sigma=0.1)$ the authors demonstrate an important difference between sub-luminal and super-luminal shocks in terms of structure and particle acceleration efficiency. In parallel shocks, the relativistic version of Bell instability is triggered and sustains an efficient DSA process. In oblique, but still sub-luminal, shocks an important contribution from the SDA mechanism was observed in competition with standard DSA. This contribution comes from the fact that the upstream plasma carries a motional electric field that can energize particles when they are reflected on the shock front. Consequently, the power-law slope of the non-thermal particle distribution function, where $\mathrm{d} N / \mathrm{d} E \propto E^{-\alpha}$, is not equal to the standard prediction but varies between 2.2 and 2.8. In superluminal configuration these authors did not find any particle acceleration. In this case shocks are mediated by the emission of semi-coherent electromagnetic wave from the shock front.

In order to illustrate the output from PIC simulations of shocks, in Fig. 22 we present the structure of perpendicular $\left(\theta_{\mathrm{B}}=90^{\circ}\right)$ relativistic shock in pair plasma for mildly magnetized case $\sigma=2 \times 10^{-3}$, obtained with the PIC code SMILEI. This structure is similar to the one found by Sironi and Spitkovsky (2009) for superluminal shocks or, more closely, to the mildly magnetized case in Sironi et al. (2013). The magnetization is chosen so that particle acceleration is efficient but maximal energy is limited by the precursor size being of the order of the Larmor radius of incoming particles $R_{L, 0}=$ $\gamma_{0} m_{e} c^{2} /\left(e B_{0}\right): \gamma_{\max } \sim 20 \gamma_{0}$. Panels (a) and (b) present the electron density in the simulation plane and the transversely averaged profile, respectively. The shock front position is delimited by the vertical dashed line and the front propagates from the right to the left side with a velocity $v_{\text {sh } \mid \mathrm{d}} \simeq 0.5 c$ as measured in the downstream (simulation) frame. Ahead of the shock front oblique filamentary density structures emerge as a result of the interaction between the incoming flow and the cloud of accelerated particles. This region defines the shock precursor. Panels (c) and (d) show the longitudinal phase space $x-u_{x}$ and transverse phase space $x-u_{y}$, respectively. The transition at the shock front is clearly seen at the position where the flow becomes isotropic and hot. The cloud of energetic particles ahead of the shock front corresponds to the accelerated population. Finally, panels (e) and (f) present the particle distribution function in energy around the shock front and far downstream, respectively. Particle acceleration operates mainly around the shock front, while far downstream distribution exhibits a Maxwellian part and the start of non-thermal tail at the highest energies. 


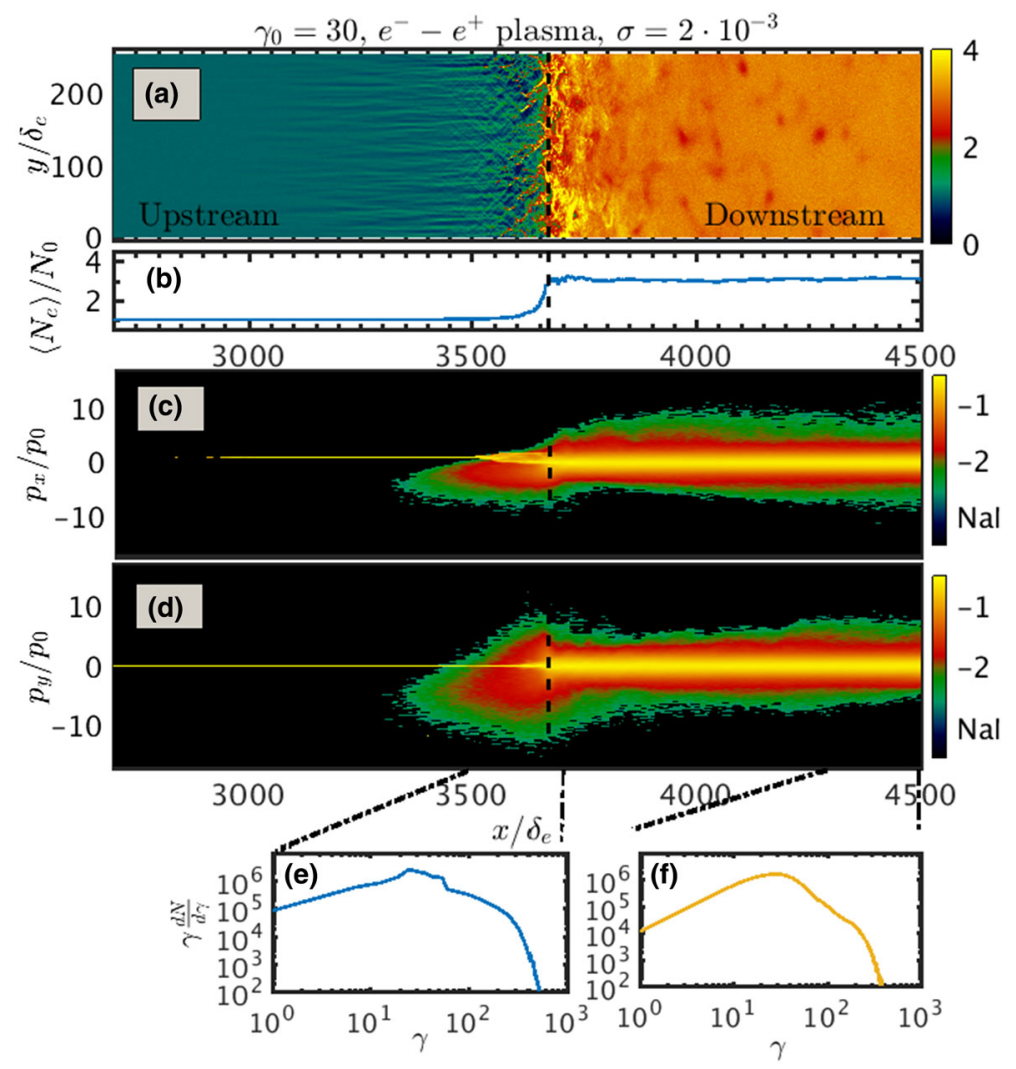

Fig. 22 Structure of a relativistic perpendicular shock in a pair plasma, at the simulation time $t \omega_{\mathrm{p}}=500$, obtained using 2D3V PIC code SMILEI. The Lorentz factor of the upstream incoming flow is $\gamma_{0}=30$ and the upstream plasma magnetization is $\sigma=2 \times 10^{-3}$. a The electron number density in the simulation plane. $\mathbf{b}$ The transversely averaged electron density normalized to the upstream value. $\mathbf{c}, \mathbf{d}$ The longitudinal phase space $x-u_{x}$ and transverse phase space $x-u_{y}$, respectively. e, f Present the particle distribution function in energy around the shock front and far downstream

Haugbølle (2011) explored the differences in the structure of unmagnetized electron-ion shocks between 2D and 3D simulations. While very similar, some quantitative differences emerged in 3D simulations: the cross shock electrostatic field is slightly larger than in 2D, magnetic energy density in the shock transition region is smaller and the index of the power-law tail is closer to 2.2 , instead of 2.4 in $2 \mathrm{D}$. The latter is more consistent with analytical expectation (e.g., Achterberg et al. 2001).

Maximal energy of accelerated particles in perpendicular shocks was investigated by Sironi et al. (2013) by means of 2D and 3D long-term simulations. Both pair plasma and electron-ion plasma were explored for a range of magnetizations from unmagnetized case $\sigma=0$ to strongly magnetized $\sigma=0.1$ and for different Lorentz factors of the upstream flow $\left(\gamma_{0}=[3,240]\right)$. The simulation box transverse size was $100 \mathrm{c} / \omega_{\text {pi }}$ in electron-positron case and $25 c / \omega_{\text {pi }}$ in electron-ion case, allowing to capture at least several filaments when Weibel-filamentation mediates the shock. It was found that the 

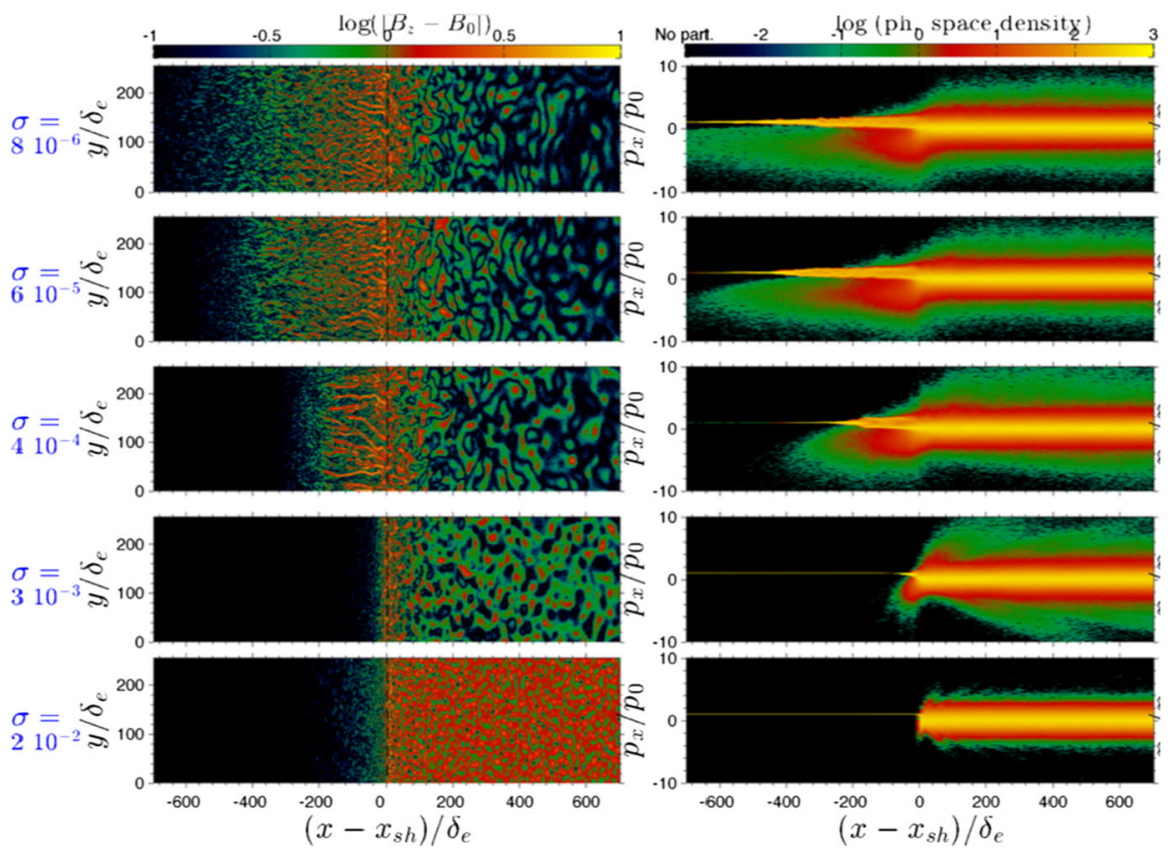

Fig. 23 Dependence of the structure of relativistic perpendicular shocks in a pair plasma on the flow magnetization $\sigma$, at the simulation time $t \omega_{\mathrm{p}}=900$. Five representative cases are shown, from top to bottom, $\sigma=8 \times 10^{-6}, 6 \times 10^{-5}, 4 \times 10^{-4}, 3 \times 10^{-3}$ and $2 \times 10^{-3}$. The left column shows the absolute value of the transverse magnetic field increment and the right column shows the longitudinal $x-p_{x}$ phase space distribution in the shock transition region. Images adapted from Plotnikov et al. (2018)

maximum particle energy increases in time as $E_{\max } \propto t^{1 / 2}$ for both electron-positron and electron-ion shocks. This result emerges from small-angle scattering regime of the accelerated particles in the self-excited micro-turbulence, where one expects the spatial diffusion coefficient to scale as $D \propto E^{2}$. The other important result of Sironi et al. (2013) study is evidencing the critical magnetization above which relativistic perpendicular shocks are not accelerating particles. For electron-positron composition the critical magnetization value is $\sigma_{\text {crit }} \approx 3 \times 10^{-3}$ and for electron-ion composition it is $\sigma_{\text {crit }} \approx 3 \times 10^{-5}$. Weakly magnetized shocks with $\sigma<\sigma_{\text {crit }}$ were found to be mediated be Weibel-filamentation that generates strong small-scale magnetic field in the vicinity of the front. In this regime DSA is efficient, with the maximum particle energy scaling as $E_{\max } \propto \sigma^{-1 / 4}$, and a fraction of energy transmitted to the supra-thermal particles $\xi_{\mathrm{CR}} \sim 10 \%$. On the other side, for $\sigma>\sigma_{\text {crit }}$ DSA is inhibited as the shock structure is no longer dominated by the filamentation instability.

Bret et al. (2014) studied the shock formation mechanism in the unmagnetized $\sigma=0$ case for pair plasma. An analytical model was developed, based on the growth and saturation time of the Weibel-filamentation instability. The formation time is estimated as a multiple of the instability $e$-folding time, $3 \tau_{\text {sat }}$. At saturation, the density in the overlap region is 2 , then the phase of density accumulation up to RankineHugoniot conditions is expected to be linear in time as the incoming plasma supplies 
the downstream region. The analytic model is then compared with 2D PIC simulation obtaining a reasonably good agreement.

Using 2D simulations, Plotnikov et al. (2018) provided a more systematic investigation over $\sigma$ than previously done, from unmagnetized to strongly magnetized shocks. Two different PIC codes were used, Finite Difference Time Domain (FDTD) and pseudo-spectral. Shock formation time, jump conditions, shock structure transition from low- $\sigma$ to high- $\sigma$ were investigated. The shock structure evolution for five different values of $\sigma$ is presented in Fig. 23. It shows the gradual transition from filamentation mediated shocks $\left(\sigma<10^{-3}\right)$, where particle acceleration is efficient, to magnetic reflection-shaped shocks $\left(\sigma>10^{-2}\right)$ where particle acceleration is inhibited, confirming the findings of Sironi et al. (2013). The shock formation time was found to be significantly longer than predicted by Bret et al. (2014). This points out the importance of other physical process than only saturation of Weibel-filamentation instability. For example, the studies of Vanthieghem et al. (2018), Ruyer and Fiuza (2018) demonstrate a dominant role of the drift-kink instability in the non-linear phase during which the shock front really forms. The particular focus of the study of Plotnikov et al. (2018) was on particle scattering properties, directly extracted by following self-consistent particle dynamics. The results demonstrated that the particle diffusion coefficient scales as $D=\left\langle\Delta x^{2}\right\rangle / 2 \Delta t \propto E^{2}$ in weakly magnetized shocks, which justifies the increase in particle maximum energy of accelerated particles as $\gamma_{\max } \propto \sqrt{t}$, evidenced by Sironi et al. (2013). In moderately magnetized shocks, the diffusion coefficient is modified by the presence of the ordered component that imposes a saturation of the maximum particle energy once particles get advected downstream under the effect of regular gyration.

\subsubsection{Mildly relativistic shocks}

The current consensus is that ultra-relativistic shocks are not very efficient particle accelerators for particle energies above $\mathrm{PeV}$ energies, mainly because of the quadratic dependence of the spatial diffusion coefficient on the particle energy, $D \propto E^{2}$. In the non-relativistic case, supernova remnants are considered to accelerate protons up to several hundreds of $\mathrm{TeV}$ or a few $\mathrm{PeV}$ at best and iron nuclei at energies 26 times higher. The question is then: how do the particles get accelerated to $10^{20} \mathrm{eV}$, maximal energy of CRs as measured at Earth? One of the promising scenarios considers mildly relativistic shocks as viable candidates (for example, trans-relativistic phases of supernova explosions or internal shocks in GRBs and jets of the AGNs). The reason is that the energy gain approaches the relativistic limit ( $\Delta E / E \simeq 1$ per cycle), while a number of intrinsic limitations of the ultra-relativistic regime are alleviated, such as the generic superluminal configuration imposed by strong contraction of the pre-shock magnetic field by the shock front. Also, recent non-linear Monte-Carlo simulations demonstrated the efficiency of trans-relativistic shocks to accelerate particles to very high energies (Ellison et al. 2013).

The mildly relativistic regime is still poorly explored with kinetic simulations as of now. In the review by Marcowith et al. (2016, section 4.3) was devoted to the discussion of mildly relativistic shocks. The studies discussed there concerned mainly the plasma 
physics of shock formation, but not the long term evolution. Here we provide a short update in light of the most recent studies.

Electron-positron plasma Using the PIC code EPOCH Dieckmann and Bret (2017), Dieckmann and Bret (2018) investigate the generation of instabilities in a 2D configuration in the case of two interpenetrating pair plasma clouds. One of the beams is produced by the reflection at a wall of the incoming beam. The simulations focus on the generation of micro-instabilities and the shock formation process, but are not long enough to investigate non-thermal particle production. Dieckmann and Bret (2018) consider a pair plasma moving at a speed of $\mathrm{c} / 2$ and perform three simulations, one in $1 \mathrm{D}$ with a resolution of $67500 \mathrm{c} / \omega_{\mathrm{pe}}$ and $3.4 \times 10^{7}$ macro particles, and two in $2 \mathrm{D}$ with the best resolution at $[67500 \times 1500]\left(c / \omega_{\mathrm{pe}}\right)^{2}$ and using 1 billion macro particles. The two-stream and Weibel instabilities are found to rule the wave growth at the shock transition layer in this regime and to take over the filamentation instability, which nevertheless may develop upstream. We note that the Debye length has to be resolved in order to accurately capture the two-stream instability, which limits the spatial and temporal extension of the simulations.

Another interesting configuration can be found in the study of the expansion of a mildly relativistic pair plasma in a background electron-proton plasma. This setup approaches the scientific case studied in the so-called two-flow model developed to investigate gamma-ray emission in blazar jets (Sol et al. 1989). Two setups have been considered either in an unmagnetized plasma (Dieckmann et al. 2018a, b) or with a guiding magnetic field oriented along the pair plasma drift direction (Dieckmann et al. 2019). While in the former work the pair were hot with a mildly relativistic temperature of $1 \mathrm{MeV}$, the two latter works have a similar setup: the simulations are 1D with a cold pair plasma with a temperature of $400 \mathrm{keV}$ moving at $0.9 \mathrm{c}$, the background plasma has a realistic proton/electron mass ratio of 1836 . The study follows the formation of the pair jet and the interaction between the two plasmas. It results from the free expansion of the pair beam the production of an electromagnetic piston that expels and compresses ambient electrons. The excess of negative current decelerates further the electrons but accelerates the positrons than can drift ahead the jet's head, and reach kinetic energies of $\sim \mathrm{MeV}$. In the meantime in both configurations (unmagnetized and magnetized) the pair beam and the background plasma interact through the filamentation instability which builds up a turbulent electro-magnetic field and contributes to accelerate the ambient protons also to $\mathrm{MeV}$ energies.

Electron-ion plasma Early studies of mildly relativistic shocks in electron-ion plasma (e.g., Dieckmann et al. 2008) presented important insights on the shock formation process but unfortunately their simulations were not long enough to follow-up on particle acceleration efficiency. The longest (and largest in transverse dimension) 2D PIC simulations to date were performed by Crumley et al. (2019) allowing the full formation and mid-term evolution of the shock. These authors studied the regime where the shock front velocity (in the pre-shock frame) is $\beta_{\text {sh }} \approx 0.83 c$, Lorentz factor $\gamma_{\text {sh }} \approx 1.8$, and the Aflvénic Mach number of $M_{\mathrm{A}}=15$. Two different magnetic field inclinations to the shock-normal where investigated: $\theta_{\mathrm{Bn}}=15^{\circ}$ (sub-luminal) and $\theta_{\mathrm{Bn}}=55^{\circ}$ (super-luminal). The main finding is that sub-luminal (quasi-parallel) shocks are effi- 
cient particle accelerators (for both electrons and ions) but not super-luminal shocks. ${ }^{42}$ When particle acceleration is efficient, the energy fraction transferred from the shock to supra-thermal ions was found to be $\varepsilon_{\mathrm{p}} \simeq 0.1$ (same as in non-relativistic and ultrarelativistic cases) and the energy fraction in accelerated electrons $\varepsilon_{\mathrm{e}} \simeq 5 \times 10^{-4}$ was found to be higher than in the non-relativistic shocks $\left(\varepsilon_{\mathrm{e}} \sim 10^{-4}\right.$; see next subsection) but still much smaller than in ultra-relativistic shocks where electrons are in equipartition with ions, hence $\varepsilon_{\mathrm{e}} \sim 0.1$.

Some details of plasma physics underlying the particle acceleration efficiency were also addressed by Crumley et al. (2019). The presence of whistler waves was found in the simulation of the quasi-parallel shock, confirming the finding of Dieckmann et al. (2008), but their role in electron acceleration or injection was found to be subdominant, i.e., with increasing mass ratio $m_{\mathrm{i}} / m_{\mathrm{e}}$ from 64 to 160 whistler dynamics were expected to play more important role in electron acceleration efficiency but this effect was not observed. The maximal energy of the accelerated particles was found to increase linearly in time similarly to non-relativistic shocks, implying that the diffusion coefficient scales as $D \propto E$, resulting from efficient excitation of Bell instability in the shock precursor. On numerical side, these authors also evidenced the importance of large transverse size of the simulation box, showing that too narrow box suppresses the electron acceleration efficiency. The reason is that too narrow box suppresses Bell modes, which dominate the non-linear physics of the shock precursor.

\subsubsection{Non-relativistic shocks}

If compared to ultra-relativistic (UR) or mildly-relativistic (MR) shocks, the difficulty of capturing the full development of non-thermal tail in non-relativistic (NR) shocks comes from the fact that the energy gain per Fermi cycle (upstream $\rightarrow$ downstream $\rightarrow$ upstream) is much smaller than in UR and MR cases, as $\Delta E / E \simeq u_{\mathrm{sh}} / c$. As a consequence, the duration of simulations must be long enough to capture at least a dozen of cycles in order to get a well-developed power-law tail while in UR shocks only a couple of cycles provides a distinguishable tail.

Despite significant efforts, only the initiation and very early stages of particle acceleration process were conveniently addressed using PIC and hybrid-PIC simulations. DSA is the most accepted model for particle acceleration at shocks. As already discussed in Sect. 2.2.3 one major difficulty for DSA to operate is the process requires particle to have a Larmor radius larger than the shock width, typically of the order of a few thermal ion Larmor radii. This concern is particularly stringent for electrons which at sub-relativistic energies have very small Larmor radii. We here discuss recent PIC simulations which address the problem of injection of electrons and ions, while the acceleration performances on dynamical timescale of the shock will be discussed in the following sections.

Table 2 presents a (non-exhaustive) list of PIC numerical experiments applied to NR shock studies. All these works are discussed in the text below.

\footnotetext{
42 We note that some caution has to be taken when assimilating super-luminal shocks with quasiperpendicular shocks. In non-relativistic cases, quasi-perpendicular-but still sub-luminal—shocks can be efficient electron accelerators.
} 


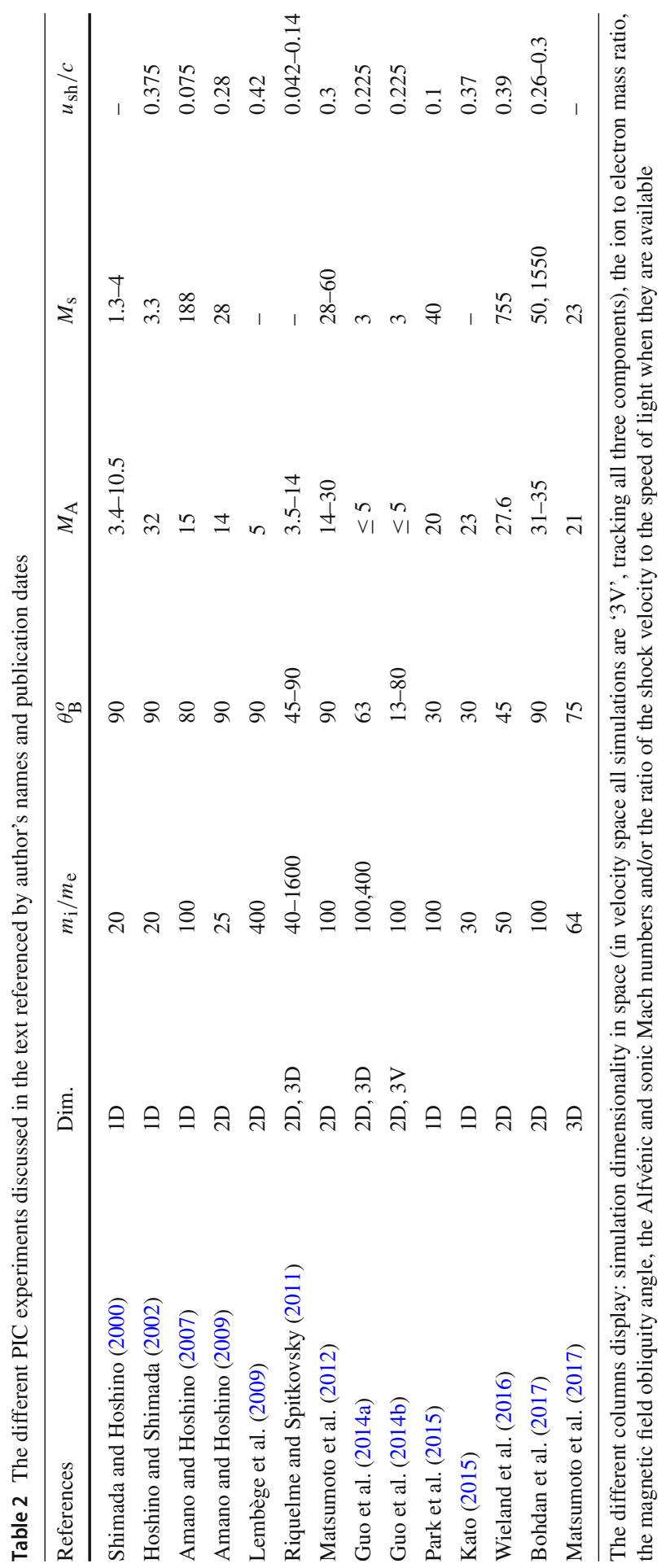


Electron injection at non-relativistic shocks Non-thermal electrons are responsible for the radio synchrotron emission from SNRs (Vink 2012) and are observed at interplanetary shocks (Masters et al. 2016). Several PIC simulations have explored the injection of electrons for different shock regimes and magnetic field obliquity.

At quasi-perpendicular shocks electrons can be accelerated by SSA if large amplitude electrostatic waves can develop at the shock front. These waves can be produced in the non-linear regime of the Buneman instability triggered by the relative streaming of reflected ions and incoming electrons (Wu et al. 1984; Shimada and Hoshino 2000). Hoshino and Shimada (2002) and Amano and Hoshino (2007) using 1D PIC simulations investigate the acceleration of electrons in a perpendicular fast shock. Electrons are heated by the interplay of the Buneman instability and trapped in these electrostatic waves and reflected back to the shock front by the motional upstream electric field. The maximum energy is expected to be at best $E_{\max }=m_{\mathrm{i}} c^{2}\left(u_{\mathrm{sh}} / c\right)$. These simulations have been generalized to multi-dimensions (2D in configuration space 3D in velocity space) by Amano and Hoshino (2009), Matsumoto et al. (2012), Dieckmann et al. (2012), Wieland et al. (2016), Bohdan et al. (2017). These works first set the criteria for SSA to occur ${ }^{43}$ : (1) the thermal speed of electrons has to be smaller than the drift speed between ions and electrons, hence the shock Mach number must satisfy $M_{\mathrm{s}} \geq(1+\alpha) / \sqrt{2} \sqrt{m_{\mathrm{i}} T_{\mathrm{e}} / m_{\mathrm{e}} T_{\mathrm{i}}}$ where $\alpha$ is the density ratio of reflected to incoming ions and $T_{\mathrm{i}, \mathrm{e}}$ are the background ion and electron temperatures, and (2) Buneman modes have to be destabilized, this requires the shock Alfvénic Mach number to satisfy $M_{\mathrm{A}} \geq(1+\alpha)\left(m_{\mathrm{i}} / m_{\mathrm{e}}\right)^{2 / 3}$. These conditions depend on the ion to electron mass ratio adopted in the PIC simulations. The development of the Buneman instability and the intensity of electrostatic waves vary considerably with the number of reflected ions in the shock reformation process. Acceleration of electrons to non-thermal energies is confirmed (but see the discussion in Dieckmann et al. 2012), but the efficiency of the process depends on the background magnetic orientation with respect to the simulation plane (recall that simulations are 2D). Acceleration is the most efficient when Buneman instability-generated waves have the highest intensities, which happens when the magnetic field is out of the plane of the simulation (Bohdan et al. 2017). Electron acceleration also depends on shock non-stationarity associated to its reformation (Lembège et al. 2009). Matsumoto et al. (2017) perform 3D perpendicular shock PIC simulations of an oblique shock $\left(\theta_{\mathrm{B}} \simeq 75^{\circ}\right)$ with a high Alfvén Mach number $\left(M_{\mathrm{A}} \simeq 21\right)$. They find a two-step electron acceleration: first electrons gain energy via SSA in the electrostatic waves driven by the Buneman instability as above, but then they further gain energy by interacting with turbulent fields produced by the Weibel ion-ion instability triggered by the interaction of reflected and background ions. The downstream electron distribution shows the formation of a power-law energy spectrum with an index $\sim-3.5$.

Riquelme and Spitkovsky (2011) perform an extensive survey of shock conditions to investigate electron acceleration. They study the effect of variations of the shock speed, ambient medium magnetization, electron to ion mass ratio and magnetic field obliquity over non-thermal electron injection at shocks. However their simulations are restricted to rather modest Alfvénic Mach numbers $M_{\mathrm{A}}<14$. One important issue

\footnotetext{
43 Bohdan et al. (2017) provide an update of these conditions for the orientation of the magnetic field with respect to the simulation plane.
} 
raised by the authors is that a small ion to electron mass ratio suppresses the propagation of oblique whistler waves (Scholer and Matsukiyo 2004), ${ }^{44}$ whereas these waves can become over-dominant to heat/energize electrons in the foot. A criterion for whistler wave to grow is $M_{\mathrm{A}} /\left(m_{\mathrm{i}} / m_{\mathrm{e}}\right)^{1 / 2}<1$ (Matsukiyo and Scholer 2003). The acceleration mechanism relies on the property of oblique whistler waves to have an electric field component parallel to the magnetic field. Particles are then first accelerated by this electric field before the complementary action of the convective electric field. Electrons are preferentially accelerated at high obliquity $\theta_{\mathrm{B}} \sim 70^{\circ}$ (at $M_{\mathrm{A}}=7$ ), where the downstream energy index is $\sim 3.6$. At smaller obliquities particles are not sufficiently confined at the shock front whereas for quasi-perpendicular shocks particles can not propagate in the foot. Electron acceleration efficiency depends mostly on the Alfvén Mach number first through the condition on whistler wave production recalled above. The electron distribution is the hardest for Alfvénic Mach numbers in the range 3-7. The energy index changes form 2.6 to 4 as $M_{\mathrm{A}}$ changes from 3.5 to 14. A complementary study of electron acceleration in low Mach number $\left(M_{a} \leq 5\right)$ shocks was performed by Guo et al. $(2014 \mathrm{~b}, \mathrm{c})$ using 2D PIC simulations in order to get better understanding of electron acceleration in galaxy cluster shocks. These authors found that a measurable fraction of incoming upstream electron (up to 15\%) bounces back upstream and formes a non-thermal tail in the distribution function with power-law index in energy $p \simeq 2.4$. These particles scatter back to the shock front on self-generated waves via firehose instability and participate in the SDA process. This acceleration process was found to be efficient if upstream plasma is high beta $(\beta \geq 20)$ for nearly any magnetic field obliquity.

High Alfvénic Mach number, quasi-parallel shocks could also allow electron injection. These shocks are likely good proton injectors (see below). In turn protons (ions) can trigger magnetic perturbations, as the magnetic field grows in the pre-shock medium then lowering $M_{\mathrm{A}}$ and its transverse component can be compressed at the front. The conditions then resemble the case of highly-oblique moderate Alfvén Mach number shocks discussed by Riquelme and Spitkovsky (2011) [see also Caprioli and Spitkovsky (2014b)]. Park et al. (2015) perform long term 1D PIC simulations of high $M_{\mathrm{A}}$ quasi-parallel shocks (see also Kato 2015). Protons destabilize non-resonant streaming (Bell) modes and electrons are accelerated by a combination of SDA and Fermi processes as they are scattered by the non-resonant streaming modes. Interestingly, non-thermal electrons entering in the relativistic regime show a $E^{-2}$ energy spectrum and a non-thermal electron to proton ratio $\sim 10^{-3}$ roughly proportional to $u_{\mathrm{sh}} / c$.

Ion injection at non-relativistic shocks The major drawback of full-PIC simulations to address the ion injection into DSA is the need to resolve both electron- and ion-scale physics, that bakes typical simulation not longer than a $\sim 10 \omega_{c i}^{-1}$. This difficulty is partly bypassed using hybrid-PIC simulations where ions are still treated kinetically but electrons are treated as massless fluid (see,e.g., Lipatov 2002; Gargaté et al. 2007; Kunz et al. 2014). In this approach all ion-scale kinetic physics are preserved while the global numerical cost is about two orders of magnitude lower than in full-PIC simulations.

\footnotetext{
44 Whistler waves are likely excited due to the cross-field drift of background electrons with respect to either reflected or background ions, this is the so-called modified two-stream instability or MTSI.
} 
The injection of thermal ions into the acceleration process was investigated by Guo and Giacalone (2013) and Caprioli et al. (2015) using multi-dimensional hybrid-PIC simulations. The first main finding of these studies is that protons are not injected by 'thermal leakage' of downstream thermalized distribution into pre-shock medium but by specular reflection on time-varying shock barrier. For quasi-parallel shocks $\left(\theta_{\mathrm{Bn}} \leq 45^{\circ}\right)$ and high $M_{a}>5$, the injection efficiency is larger than $10 \%$. As evidenced by Caprioli et al. (2015), protons gain energy through SDA in consecutive reflections on the shock front and inject into DSA when their energy is large enough to escape upstream. They also propose a quantitative model that accounts for the drop in injection efficiency of quasi-perpendicular shocks with $\theta_{\mathrm{Bn}} \geq 45^{\circ}$, as more than 4 SDA cycles are required for injection into DSA, while at each SDA cycle a large fraction of ions $(\sim 75 \%)$ is lost downstream. This effect explains the rapid drop in injection efficiency of $\theta_{\mathrm{Bn}} \geq 45^{\circ}$ shocks (see, however, Ohira 2016).

Other studies addressed the thermalization of heavy ions in post-shock medium and chemical enhancement in shock accelerated particles. It was found that each species acquires downstream temperature proportional to its mass, $T_{d} \propto A_{i}$, where $A_{i}$ is the atomic number (Kropotina et al. 2016; Caprioli et al. 2017). The efficiency of ion injection into DSA increases with $A / Z$ ratio, where $Z$ is the charge. Caprioli et al. (2017) show that there is preferential acceleration of ions with large $A / Z$ in quasiparallel shocks. For $M_{a}>10$ these authors find that the fraction of DSA-accelerated ions scale as $(A / Z)^{2}$, in quantitative agreement with abundance ratios in Galactic Cosmic Rays. The injection mechanism of heavy ions is different from proton injection, since they do not have any dynamical impact on the shock structure. Instead of reflecting specularly on the shock front, heavy ions directly thermalize in the postshock medium. If the downstream isotropization time is shorter than advection time, a small fraction can back stream into pre-shock medium and participate in DSA. We note that Hanusch et al. (2019), using 2D hybrid-PIC simulations as well, confirm the preferential injection of heavy ions up to $A / Z \sim 10$ but find that there is saturation for higher $A / Z$ values, in contrast with Caprioli et al. (2017) findings. For quasi-perpendicular shocks with $\theta_{\mathrm{Bn}}>50^{\circ}$, similarly to pure electron-proton shocks (Caprioli and Spitkovsky 2014a), there is no injection into DSA of any ion species, since advection time becomes shorter than isotropization time.

\subsubsection{Discussion}

Let us summarise and briefly discuss the micro-physical studies of particle acceleration at collisionless shocks. Several key points emerge in recent studies:

- The self-consistent shock structure produces non-thermal particle distributions for a broad range of parameter space. The main requirement for efficient particle acceleration is the ability of particle impinging the front to escape back into the pre-shock medium and trigger wave growth through kinetic instabilities. This condition is generally met in high-Mach number sub-luminal shocks (or weakly magnetized shocks).

- Unmagnetized relativistic shocks are efficient particle accelerators in both electron-positron and electron-ion plasma. Here, it was demonstrated that the 
Weibel-filamentation instability mediates the shock transition region. It leads to significant magnetic field amplification and concomitant particle acceleration. Typically, about $1 \%$ by particle number fraction and $10 \%$ by the shock kinetic energy fraction is channelled to non-thermal particles. However, the acceleration rate is slow as it scales quadratically with particle energy: $t_{\text {acc }} \propto E^{2}$. Thus, one infers the maximum energy of protons achievable in ultra-relativistic shocks of GRBs as $E_{\max } \sim 10^{16} \mathrm{eV}$.

- When the relativistic shock front propagates into highly magnetised medium ( $\sigma>$ 0.01 ) one has to distinguish between sub-luminal and superluminal configurations. In the former case, particle acceleration was found to be efficient, sometimes even more than in unmagnetised case. Yet, the sub-luminal configuration is statistically disfavoured in relativistic case. When the configuration is superluminal, shockprocessed particles are advected downstream and are unable to undergo Fermi process.

- At intermediate magnetization, $\sigma_{\text {crit }}<\sigma<0.01$, limited particle acceleration occurs and the maximum energy scales as $E_{\max } \propto \sigma^{-1 / 4}$.

- The interesting case of mildly-relativistic shocks, e.g., $\gamma_{\mathrm{sh}} \geq 1$, where energy gain per Fermi cycle is large and the shock can easily be subluminal is poorly studied. Recent study by Crumley et al. (2019) found that shock physics in quasi-parallel case is similar to non-relativistic shocks.

- Non-relativistic shocks are the most common and studied in literature. The most representative case is the external shock of the Supernova Remnants. In this regime, when particles are efficiently reflected on the front, several instabilities can be in competition in the precursor region (e.g., Buneman, firehose, Whistler, Weibel, gyroresonant, Bell), depending on the Mach number, magnetic field strength and obliquity. Hence, the phenomenology is more complex then in ultra-relativistic shocks. For example, quasi-parallel shocks are common, contrary to the ultrarelativistic regime, and lead to efficient magnetic field amplification through resonant and Bell instability in the shock precursor.

There are several open questions under active investigation or to be addressed in the near future.

1. Are ultra-relativistic shocks always locked in the slow acceleration rate, i.e., $t_{\text {acc }} \propto$ $E^{2}$, or an additional source of magnetic turbulence can produce faster acceleration? How is the long term evolution of unmagnetized shocks where the shock transition is governed by self-excited microturbulence ? No steady state was reached with current simulations. For recent progress in this field, see Lemoine et al. (2019a).

2. The question of which configuration, quasi-parallel or quasi-perpendicular, in nonrelativistic shocks is more efficient for ion/electron acceleration? The regime of quasi-perpendicular but still sub-luminal shocks is a particular case that requires clarification.

3. How promising is the mildly relativistic regime?

4. On numerical side, important efforts are undertaken to push PIC simulations to the largest scales and longest time benefiting from modern computational resources. While largely needed, gaining one order of magnitude in system size and simulation time becomes rapidly prohibitive even with the largest available supercomputers. 
In this respect, hybrid approaches such as MHD-PIC are promising when one is mainly interested in dynamics of supra-thermal particles (see Sect. 5.5). Yet, this requires to robustly prescribe how some part of thermal particles (simulated using the fluid approach) are promoted to non-thermal status (simulated using PIC or Vlasov approach). For example, in the shock problem one prescribes a fixed fraction of shock-processed particles to be injected into the non-thermal pool (Bai et al. 2015; van Marle et al. 2018) but more accurate parametrisation is required when the shock structure becomes modified by non-thermal particles.

In conclusion, PIC simulations provide detailed non-linear solutions of the shock problem. Therefore, they are an efficient tool to probe the efficiency of particle acceleration for a given parameter set. They are, however, of limited duration (a couple of ion gyro-periods for full-PIC and $\sim 10^{3}$ ion gyro-periods for hybrid-PIC) and box sizes are typically less than thousands of ion skin depths. Even with the most powerful current computational facilities, simulating the global astrophysical systems is unachievable with this approach. The goal and common approach is to provide robust scalings which can be included in fluid simulations as sub-grid prescriptions.

\subsection{Kinetics of magnetic reconnection}

There is a vast literature on magnetic reconnection, both for the collisional case based on resistive MHD and the non-collisonal case based on the Maxwell-Vlasov equations. Nevertheless, many questions are still far from understood, including 'what triggers reconnection events in real astrophysical objects', 'what are the physical processes which accelerate particles to super-high Lorentz-factors', 'do associated energy spectra always show power-law slope and what is the spectral index', 'can ions be accelerated to equal energies as electrons', 'is there an upper limit for the Lorentz-factors that can be achieved and which process sets this upper limit'? A deeper understanding of these questions will definitely help to answer relevant astrophysical questions: (1) To what degree is magnetic reconnection important for the dynamics of large scale flows like the launching of jets from compact objects or driven shock waves? (2) To what degree is REC responsible for the production of thermal and nonthermal high-energy photons observed from the Sun to AGNs? (3) To what degree can REC accelerate ions to relativistic speeds and can thus contribute to the cosmic ray flux and the hadronic channel of emissivity of photons and energetic neutrinos?

As the literature is vast there is no chance to refer to all papers. In a hopefully not too biased view, basic ideas are thus presented on the basis of selected papers in 4 subsections. (1) What kinetic simulations can achieve and why we decisively need them, (2) Some key results based on kinetic simulations, (3) The most prominently discussed physical processes able to accelerate particles, (4) A critical discussion and outlook. For further important points, which are not discussed due to lack of space, we refer to the reviews given at the beginning of Sect. 2.5.

\subsubsection{What kinetic simulations can achieve and why we decisively need them}

Microphysical studies have the great advantage that they rely only on fundamental physics. Difficult questions-like which equations of the MHD family best 
model reconnection events and which values of the transport coefficients (resistivity, viscosity, Hall-parameters, or even higher moments) are most appropriate-can be omitted. All this comes self-consistently from the kinetic physics solved, described by Vlasov-Maxwell equations given in Sect. 3.1. The numerical method most widely used in astrophysics is the PIC method described in Sect. 3.3, though solvers for the Vlasov equations in the $6+1$ dimensional phase space start to appear.

The price to pay is that the computational costs to solve kinetic equations are much higher than those to solve any MHD model-even complex ones like the popular 10moment closure model, see for instance Wang et al. (2015) or Lautenbach and Grauer (2018). With kinetic simulations, even huge ones, only local aspects can be addressed, on spatial scales which measure at most some thousand electron inertial lengths and thus up to about hundred proton inertial lengths. This is sufficient to study a single $\mathrm{X}$-point or even a small current sheet breaking up into a plasmoid chain. It is, however, not sufficient to answer questions like how these reconnection sites are embedded in the large scale environment and how they have been formed. One way out, which is included in the discussion below, is to apply some splitting between MHD models and localized particle models or to post-process MHD solutions by propagation of test-particles. In the future, more numerical codes will probably be available which dynamically couple MHD and particle dynamics. Some references to such codes are given at the end of Sect. 2.5.

Another limitation of kinetic simulations is that they do not include photons and particle physics. Such aspects are ultimately relevant for high-energy plasma processes. Power law slopes and cutoffs may change when considering energy losses by the emission of synchrotron radiation or inverse Compton scattering of electrons on colder photons originating from the reconnection site itself or from other, external processes, possibly far away from the reconnection site. In relativistic REC, where the energy involved exceeds the rest masses of electrons (and maybe even of protons), the building of electron-positron-pairs is very likely to take place, which significantly back-react on the reconnection dynamics. The same may be true if accelerated protons can create pions and higher hadronic resonances and neutrinos. Attempts to account for radiative losses have recently been made, but are only at the very beginning.

Despite these limitations, kinetic simulations have brought, in about the last 10 to 15 years, an immense progress in our understanding of both, magnetic reconnection and shock waves, as well as of associated particle acceleration. This progress could not have been achieved on the basis of pure MHD simulations.

Simulation setup Most kinetic simulations of magnetic reconnection have been performed in two spatial dimensions or in simple prolongations into the third dimension (but see the paragraph on dimensionality) of the setup sketched below. In two spacedimensions, there are known two analytical, stationary, though unstable configuration of current sheets for the Vlasov-Maxwell equations. These solutions are typically used to setup a kinetic simulation to study magnetic reconnection.

(1) Harris-sheet: the reconnecting magnetic field $B_{0}$ is oppositely oriented along one axis (say the x-axis in Fig. 24). Normal to it (along the $y$-axis), the field strength varies as $\mathbf{B}_{\mathrm{x}, \mathrm{rec}}(y)=\hat{x} B_{0} \tanh (y / \delta)$. The thickness of the sheet, $\delta$, typically is a few electron inertial lengths and thus, for realistic mass ratios between 


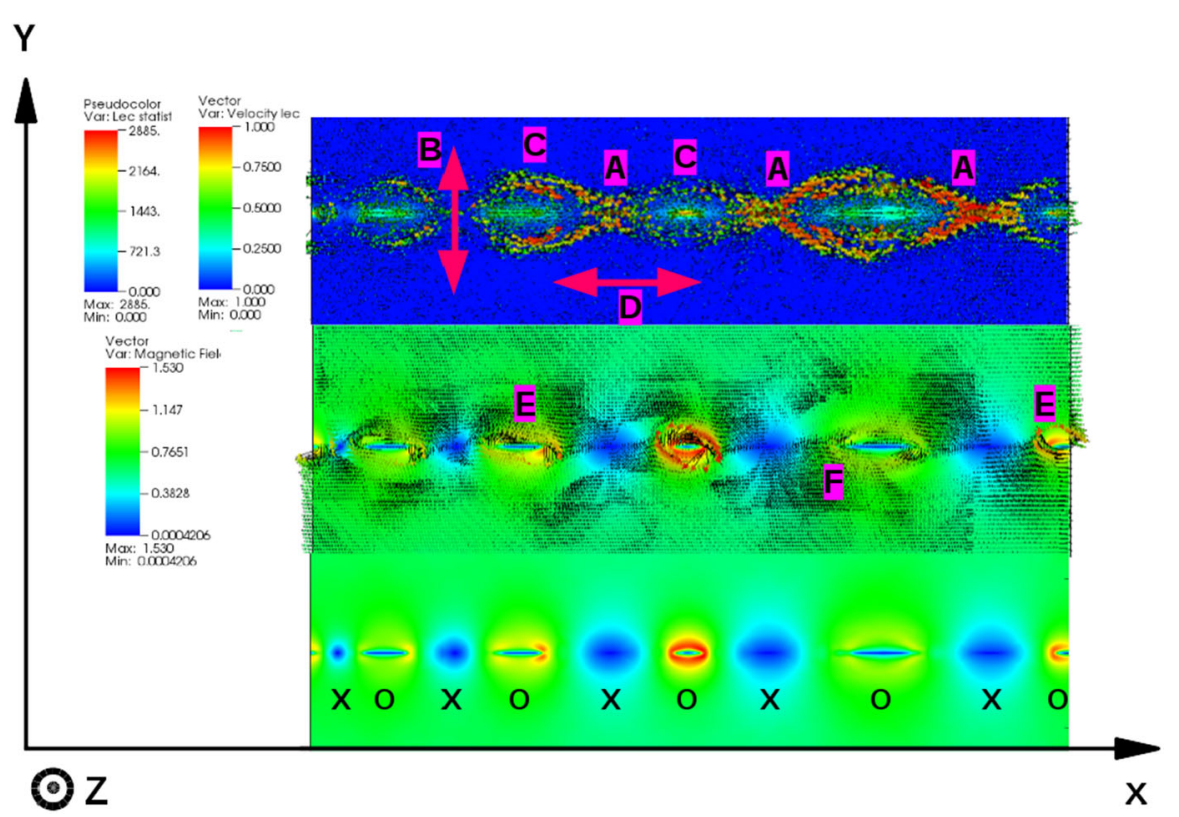

Fig. 24 Acceleration sites in a Harris-sheet broken into a chain of plasmoids and X-points (from an unpublished PIC simulation by D. Folini and R. Walder). Shown are accelerated electrons on top of the electron number density (top panel), magnetic field orientation (middle panel), and magnetic field strength (bottom panel). Possible acceleration sites are indicated by different labels: (A) Reconnecting electric field. (B) First order Fermi-mechanism between the converging inflows to the reconnection site. (C) Contracting plasmoids. (D) Merging plasmoids. (E) Drifts in inhomogeneous magnetic fields. (F) Turbulence

electrons and ions, less than a proton inertial length. The plasma density varies as $n(y)=n(0) / \cosh ^{2}(y / \delta)$ along the $\mathrm{y}$-axis. Together with an appropriate temperature, the thermal pressure within the sheet balances the magnetic pressure from outside the sheet. The induced current then points into the normal direction and writes $J(x)=c B_{0} / 4 \pi \delta \operatorname{sech}^{2}(x / \delta) \hat{z}$.

(2) Force free equilibrium: a basically similar equilibrium can be obtained using the force-free equations (see, e.g., Guo et al. (2015); Wilson et al. (2017) for details).

The parameters of both configurations are symmetric with respect to the center of the sheet, except that the direction of the magnetic field is reversed. Some authors use for their setup many such current sheets aligned in parallel, (see Biskamp 2000; Drake et al. 2010; Kowal et al. 2011; Kagan et al. 2013; Werner et al. 2018). This can be quite natural, for instance within the frame of striped wind models of the solar corona ( $\mathrm{Li}$ et al. 2014) or at the termination shock of the solar wind at the heliopause (Drake et al. 2010) or in neutron star nebulae (Pétri and Lyubarsky 2007; Kirk et al. 2009; Sironi and Spitkovsky 2012; Uzdensky and Spitkovsky 2014; Cerutti et al. 2016; Cerutti and Philippov 2017).

An extension, and a step towards more realistic models, is to use asymmetric initial configurations (Cassak and Shay 2007; Belmont et al. 2012; Hesse et al. 2013; Aunai et al. 2013; Pritchett 2013; Eastwood et al. 2013). Within this review we do not further 
elaborate on this situation. We concentrate on the 2D Harris-sheet instead, what can be learned from it and where the limitations of this toy setup lie.

If the thickness of such current sheets is less than a typical field diffusion length, they are linearly unstable to tearing modes. Eventually, X-points will develop where REC will start. In simulations, one often uses overpressured inflows to accelerate the development of the instability. As described below, each X-point will develop its exhausts. Exhausts of different X-points may collide to form magnetic islands (also called plasmoids or O-points), see Fig. 24. Waves are generated and with them turbulence develops within the sheet and outside of it, on both its sides in the diffusion regions. These ingredients turn out to be important drivers of non-thermal particle acceleration.

Some authors trigger one single X-point in their simulations, from which huge exhausts develop. Within the exhausts, secondary X-points and islands can develop. Many features described below are valid for both, the triggered and the un-triggered approach. Both approaches develop a power-law spectrum of accelerated non-thermal particles (Figs. 25, 26, 27). Differences between the two approaches and whether one approach is more close to a reconnection event in astrophysics are not yet worked out in detail (but see some points discussed below).

Different boundaries can be used and these will be discussed when presenting simulations and associated results below.

The parameters governing the physics It turns out that the evolution of the sheet and the acceleration of particles depend on the strength of the reconnecting magnetic field, the particle density, and the field temperatures of electrons and ions. Often, the magnetization is given as the ratio $\sigma_{\mathrm{s}}$ of magnetic energy density to enthalpy density, where $s$ stands for the particle species, either electrons or ions, $s=e, i$. To account for temperature effects, one may define $\beta_{\mathrm{s}}$ as the ratio of particle thermal pressure and magnetic pressure. Other, equivalent, characterizations used are $\sigma_{\text {hot,s }}$, the ratio of the energy flux in the reconnecting magnetic field to that in the particles for each species (thermal and bulk, and particle rest mass) and $\sigma_{\text {cold,s }}$, which does not consider temperature and bulk flow.

A particular parameter is the background field. The term refers to the field component which is not going to reconnect, i.e., a component which encloses a certain angle, $\theta_{\mathrm{B}}$, with the inverted field components that drive the reconnection process. If a background field is present, the reconnection physics is going to alter drastically.

As discussed in Sect. 2.5, the physics of collisionless REC is different for pair plasmas on the one hand and for an electron-ion plasma on the other hand. The much larger masses of ions as compared to those of electrons results in their much earlier de-magnetization when the plasma flows into the reconnection region (consult Fig.10), with a series of consequences (Melzani et al. 2014b). Relativistic settings also result in peculiarities (Melzani et al. 2014a, b). In the ultrarelativistic limit (i.e., when for both, electrons and ions, the energy largely exceeds the rest-mass), plasma time- and lengthsscales (cyclotron frequencies, skin depths, Larmor radii) become independent of the particle rest mass and depend only on the particle energy. Guo et al. (2016) showed that, in this ultra-relativistic limit, a pair and ion/electron plasma behave essentially similarly. 

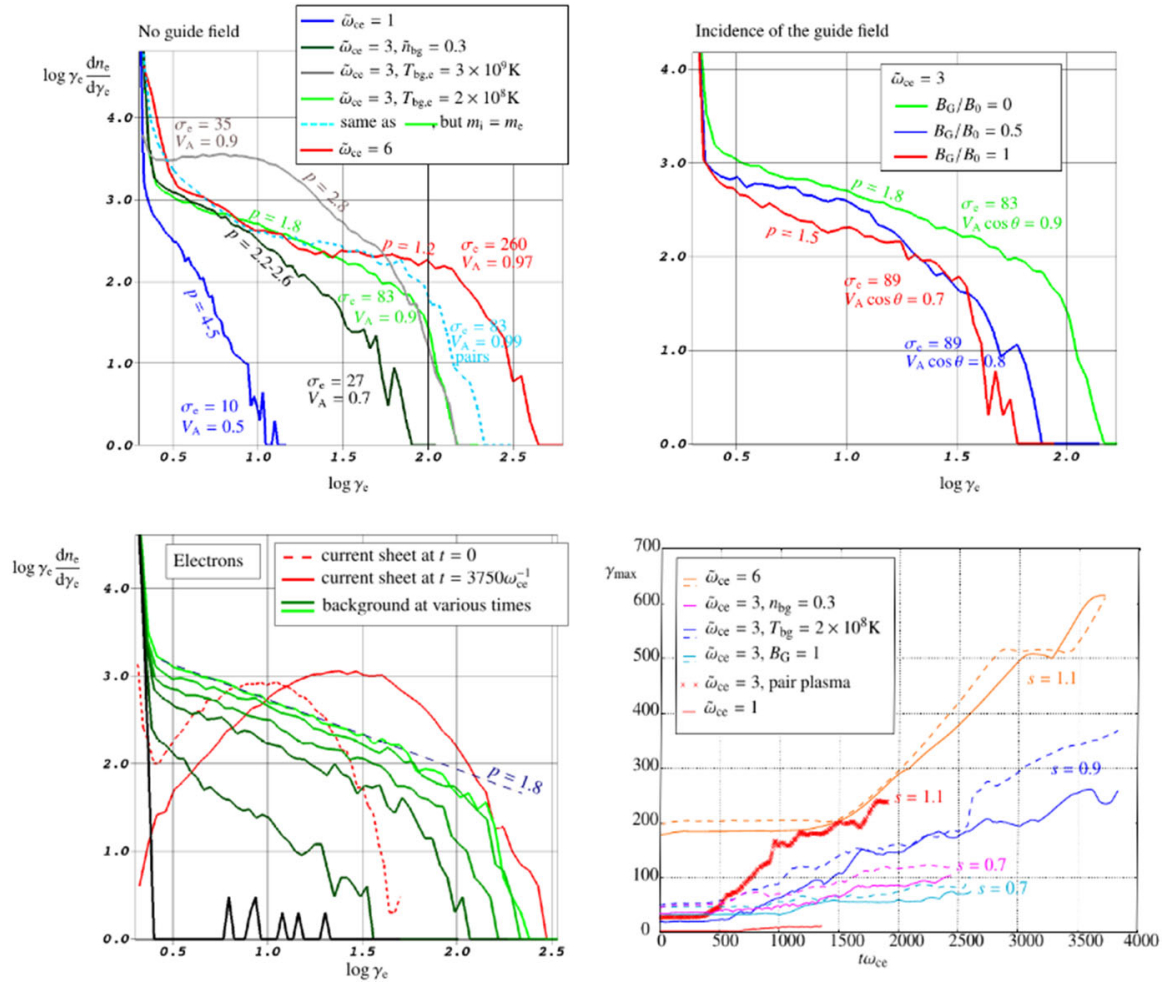

Fig. 25 Upper row: final electron spectrum for various simulations, without (left panel) and with a guide field (right panel). Lower row, left panel: Lorentz factor distributions of electrons of a simulation by Melzani et al. (2014b), using $\omega_{\text {ce }} / \omega_{\text {pe }}=3$ and a magnetization in the background plasma $\sigma_{\text {hot,i,e }}=3.6,83$. Red curves indicate particles originally found in the setup current sheet, green curves indicate particles from the plasma which flows into the sheet during the reconnection process. For the green curves, times are ordered as dark to light green, with values $0,750,1500,2250,3000,3750 \omega_{\mathrm{ce}}^{-1}$, i.e., one curve every $750 \omega_{\mathrm{ce}}^{-1}=250 \omega_{\mathrm{pe}}^{-1}=50 \omega_{\mathrm{pi}}^{-1}=30 \omega_{\mathrm{ci}}^{-1}$. The blue dashed line indicates the final power-law slope of the background accelerated particles. Lower right panel: the time-evolution of the maximum Lorentz factor of the background particles for various simulations with $m_{\mathrm{i}} / m_{\mathrm{e}}=25$ or 1 . Solid lines are for electrons, dashed lines for ions and represent $m_{\mathrm{i}} / m_{\mathrm{e}} \gamma_{\mathrm{i}, \max } \cdot \tilde{\omega}_{\mathrm{c}, \mathrm{e}}=\omega_{\mathrm{c}, \mathrm{e}} / \omega_{\mathrm{p}, \mathrm{e}}$. The index $s$ refers to the power law index $t^{\mathrm{s}}$. Images adapted from Melzani et al. (2014b)

Regarding PIC simulations themselves, care must be taken that the number of superparticles per discretization cell is sufficiently high to ensure that collisionless kinetic processes remain faster than collisional effects, e.g., for thermalization. For this, the PIC-plasma parameter, $\Lambda^{P I C}$, the number of superparticles per Debye sphere, has to be sufficiently large, see Melzani et al. (2013) for a more thorough discussion. In addition, when using an explicit PIC scheme on a Yee grid (in particular of low order), care must be taken that energy conservation is sufficiently well guaranteed and that artificial Cherenkov radiation (Greenwood et al. 2004) does not dominate the scene. The problem appears in particular for particles traveling close to the speed of light, where the high-frequency waves from artificial Cherenkov radiation actively interact with the particles and thus may influence the acceleration process in a non-physical way (see section 3.3.3). 

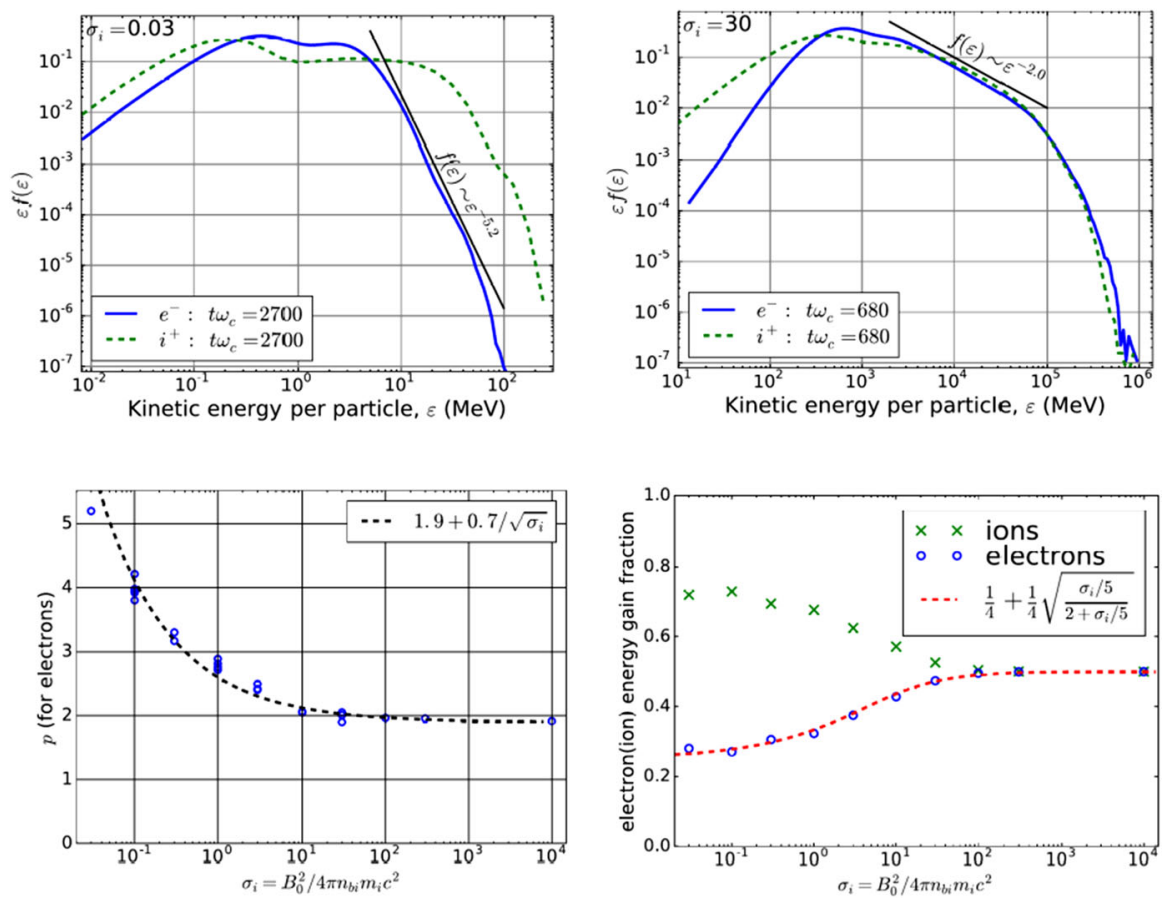

Fig. 26 Upper row: final electron (blue-solid) and proton (green-dotted) spectrum of non-thermal particles for a low $\left(\sigma_{i}=0.03\right.$, left panel $)$ and a high magnetization $\left(\sigma_{i}=30\right.$, right panel). Lower left panel: fit for the electron power law index $p$ (Eq. 46). Lower right panel: final energy partition between background electrons and ions at different ionizations $\sigma_{\mathrm{i}}$. As expected, there is no difference between electrons and ions in the ultra-relativistic regime. The fit relates to Eq. (45). Images adapted from Werner et al. (2018)

\subsubsection{Some key results based on kinetic simulations}

In this section, selected results are presented regarding the energy spectra of accelerated particles, the energy partitioning between ions and electrons, and the overall efficiency of the energy transfer from the reconnecting magnetic field to the accelerated particles. These results set the stage for a more detailed view on the physical mechanisms behind particle acceleration, to be presented in the next section.

We concentrate on the case of a relativistic electron-ion plasma in an idealized 2D Harris-sheet set up. The non-relativistic case is important to understand processes on the Sun, space-weather, but also technical devices, from plasma thrusters to tokomaks. The case of relativistic pairs is interesting in pulsar winds and perhaps in certain regions of a black hole corona-in every environment where pair-cascades could develop. However, these two cases will not be discussed due to lack of space and because many results have been presented in other reviews (references given at beginning of Sect. 2.5).

We call REC semi-relativistic when the energy of the magnetic field exceeds the electron rest mass energy but is smaller than the ion rest mass energy, otherwise we 

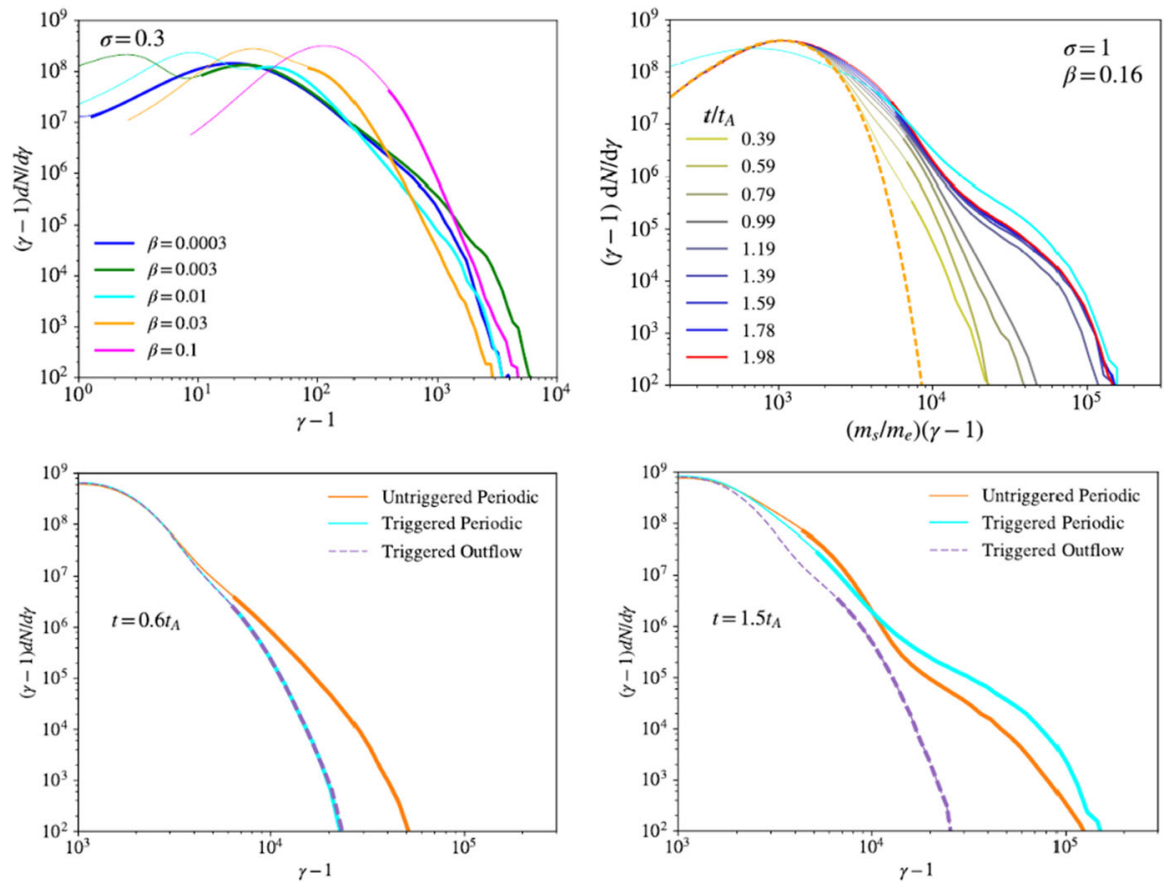

Fig. 27 Upper left panel: the $\beta_{\mathrm{i}}$-dependence of the final $\left(t / t_{\mathrm{A}}=2\right)$ electron spectra at low magnetization. Upper right panel: time-evolution of the electron spectrum for $\sigma_{\mathrm{i}}=1$ and $\beta_{\mathrm{i}}=0.16$. The spectrum starts to develop an additional component after about one $t_{\mathrm{A}}$. Lower panels: electron spectra for different initial and boundary conditions. For details, see text. Image adapted from Ball et al. (2018)

call it relativistic. Ultra-relativistic REC terms the situation where the magnetic energy largely exceeds the rest mass energy of both species, electrons and ions.

There are only a few papers that have addressed the relativistic electron-ion regime though it is decisive for our understanding astrophysical high-energy objects: the dynamics and emission of accretion disks around and jets from either black holes or neutron stars (see Table 3).

Melzani et al. (2014a) and Melzani et al. (2014b) present a first study of collisonless relativistic magnetic reconnection of an electron-proton-plasma in two space dimensions. They use reduced mass ratios $m_{\mathrm{p}} / m_{\mathrm{e}}=25$ and 50 respectively, and started from a Harris-equilibrium. Periodic boundary conditions normal to the current sheet and reflecting conditions parallel to the sheet are used. Tearing instability and subsequent REC develop from numerical noise. The magnetization varies as $10 \leq \sigma_{\mathrm{e}} \leq 260$ and as $0.4 \leq \sigma_{\mathrm{i}} \leq 14$. Except for one simulation $2.8 \cdot 10^{-5} \leq \beta_{\mathrm{p}, \mathrm{i}}=\beta_{\mathrm{p}, \mathrm{e}} \leq 2 \cdot 10^{-3}$. They present cases without a guide field and with a guide field of $B_{\mathrm{G}}=0.5 B_{0}$ and $B_{\mathrm{G}}=B_{0}$, where $B_{0}$ is the reconnecting component of the field, the component (anti-)parallel to the current sheet.

Melzani et al. (2014a) describe the general structure of the reconnection process for both, the cases with and without guide field. The bulk inertia is identified as the main 


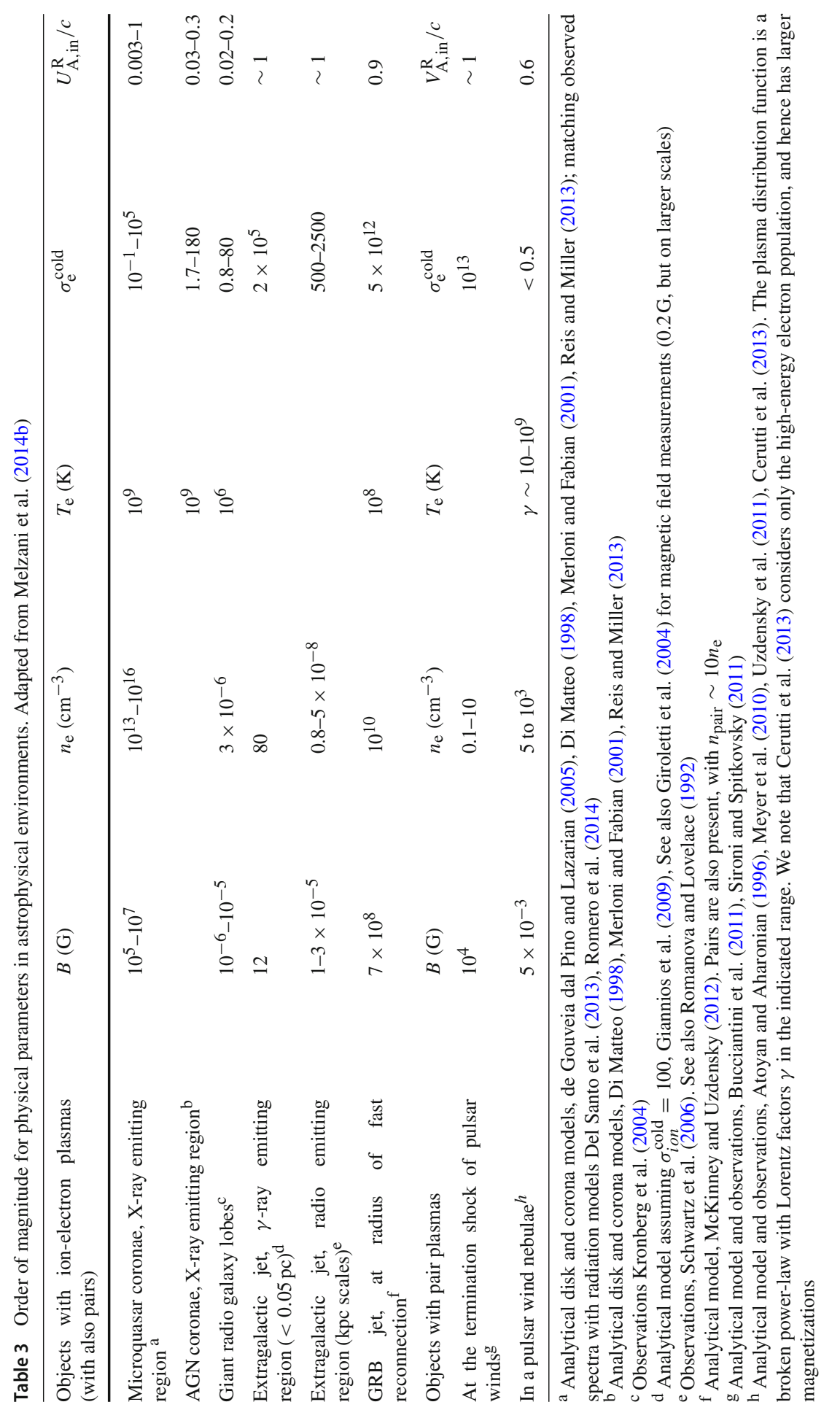


non-ideal process, which de-magnetizes both, electrons and ions. In the energy flux of the exhausts the thermal component dominates over the bulk component. Protons are generally hotter than electrons in the exhausts. The numerical results correspond to analytical estimates given. They identified a good measure for the relativistic reconnection rate,

$$
E^{*}=E_{\mathrm{Rec}} / B_{0} U_{\mathrm{in}}^{\mathrm{R}},
$$

with $U_{\text {in }}^{\mathrm{R}}$ the relativistic Alfvén speed, $B_{0}$ the reconnecting field, and $E_{\mathrm{Rec}}$ the reconnection electric field. This rate varies between 0.14 and 0.25 , showing higher values with lower background densities. Generally, the rate is higher than in the non-relativistic case (for which $0.07 \leq E^{*} \leq 0.14$ ), which is in line with the findings for pair plasmas, e.g., by Zenitani and Hoshino (2007) $\left(E^{*}=0.2\right)$, by Cerutti et al. (2012b) $\left(E^{*}=0.17\right)$, or by Bessho and Bhattacharjee (2012) $\left(E^{*}=0.19\right.$ and 0.36$)$. It follows directly that the reconnection electric field is very large, $E_{\mathrm{Rec}} / B_{0} \sim 0.2 U_{\mathrm{A} \text {, in }}^{\mathrm{R}} \sim 0.2 c$, which turns out to be important for the acceleration process of particles.

Melzani et al. (2014b) further elaborate on associated results, notably on the acceleration of non-thermal particles to very high Lorentz-factors. The results are summarized in Fig. 25.

REC produces a population of non-thermal particles. This population shows a power-law spectrum, which gets harder for increasing magnetization (upper left panel). To first order, the slope of the power law is independent of the presence of a background field (upper right panel). There is a clear difference in the spectrum of accelerated particles (lower left panel), depending on whether the particles have been present in the initial current sheet (population $C S$ ) or whether particles have floated into the sheet after REC has started (population BG). Population $B G$ forms a power-law, population $C S$ is substantially heated but its spectrum is still Maxwellian. This points to a different acceleration mechanism of the two populations, see next section.

The lower right panel of Fig. 25 shows the temporal evolution of the maximum Lorentz-factor $\gamma_{\max }(t) \sim t^{\mathrm{s}}$, which is close to an exponential growth. Higher magnetization $\sigma$ in a larger coefficient $s$ and thus a faster growth of the spectrum to high Lorentz-factors. While, as said above, non-thermal accelerated particles have the same spectrum, independently whether a background field is present or not, the growth time of the power-law cutoff $\gamma_{\max }$ is clearly slower in the presence of a background field. Also, to first order, $\gamma_{\max }$ for protons grow slower than electrons by a factor $m_{\mathrm{i}} / m_{\mathrm{e}}$.

Finally, while in the thermal exhausts there is much more energy in the ions than in the electrons, the situation is slightly different for the accelerated particles. Without a background field, there is more energy in the ions. But the situation reverses when a background field is present, when more energy is carried by the electrons. We add a note of caution to this result as the mass-ratio between electrons and ions used is either 25 or 50 . Simulations with a realistic mass ratio should be undertaken and their results be confronted with the findings of Melzani et al. (2014b).

Guo et al. (2016) perform PIC simulations of relativistic electron-ion reconnection (magnetically dominated in their terms) without a guide field, starting from a force-free equilibrium of the current sheet. They use different mass-ratios between electrons and ions, between 1 and 1836 and use different domain sizes and different inflow temperatures. They explore magnetizations reaching from the relativistic to the 
ultra-relativistic case, but cover not really the semi-relativistic case, in contrast to the other work discussed in this section. This should be kept in mind when looking at the following results. They find that for low mass ratios, ions gain slightly (1.1 times) more energy than electrons while for a real mass ratio, the ions gain 1.5 times more energy than the electrons. All power-law slopes are hard - when put into the form $f \propto(\gamma-1)^{-s}, s$ is between 1 and 2 and very close to 1 for high magnetizations. For their high magnetizations, the electron power-law slope does not depend on the mass ratio and is the same for a pure pair plasma, for a mass ratio of 100 and for a realistic mass ratio. In energy space, the slopes for electrons and ions slightly differ, e.g., 1.35 for electrons and 1.2 for ions for a simulation with $\sigma_{0}=B_{0}^{2} /\left(4 \pi\left(m_{\mathrm{i}}+m_{\mathrm{e}}\right) n c^{2}=100\right.$ ( $n$ is the particle number density) and $m_{\mathrm{i}} / m_{\mathrm{e}}=100$. However, the momentum distribution shows $s_{\mathrm{p}}=1.35$ for both species. They argue that this can only be achieved by a Fermi-like acceleration mechanism. They find a slight dependence of the power laws on the size of the current sheet, not so much for the all over slope, but for secondary variations of the slope. Smaller domain sizes show more variations, with a significant change of the slope for different energies $\left(\gamma_{\mathrm{e}}-1\right)$. Larger domain sizes show a much more smooth, unique slope over the entire energy range. Finally, these authors emphasize that all their power-laws show an exponential cutoff.

Werner et al. (2018) present an extensive 2D study of collisionless REC in the semi-relativistic and relativistic regime, varying the ion magnetization, $\sigma_{i}$, from $10^{-3}$ to $10^{4}$. The plasma-beta value of ions was 0.01 for all simulations. A realistic mass ratio between ions and electrons is used. For their setup, they use a doubled Harris sheet with two field reversals an they use period boundary conditions in both directions. The authors analyze the plasma flows in the 'thermal' regime and describe the Hall signatures due to the different sizes of the electron and ion diffusion region (see Fig.10). They find a reconnection rate of about 0.1 of the Alfvénic rate (their slightly different definition of the reconnection rate) across all regimes, slightly below (0.08) in the semi-relativistic regime and slightly above (0.12) in the ultra-relativistic regime. In the ultra-relativistic limit, the release of magnetic energy during REC is distributed equally between electrons and protons, but protons gain more in the semi-relativistic regime, up to $75 \%$ for the weakest $\sigma_{i}$ (see Fig. 26, lower right panel). The authors present a formula for the fractional energy gain of the electrons, $q_{\mathrm{e}}$,

$$
q_{\mathrm{e}}=\frac{1}{4}\left(1+\sqrt{\frac{\sigma_{\mathrm{i}} / 5}{1+\sigma_{\mathrm{i}} / 5}}\right) .
$$

Integration is done till 2000 Larmor time-scales. Particles are accelerated to a non-thermal regime. Power-laws and energy-cut off seem to saturate till the end of the simulation. By fitting their data to a power-law, they find for electrons the timesaturated relation

$$
f(\epsilon) \sim \epsilon^{-p} ; \quad p\left(\sigma_{\mathrm{i}}\right) \approx 1.9+0.7 \sigma_{\mathrm{i}}^{-1 / 2}
$$

where $\epsilon$ denotes the kinetic energy of the electrons without their rest-mass (see Fig. 26, lower left panel). They emphasize that this index can be understood on the basis of the 
bouncing of electrons between approaching islands (see next section). The normalized cutoff energy $\epsilon_{\mathrm{c}} / \sigma_{\mathrm{e}} m_{\mathrm{e}} c^{2}$ rises slowly with $\sigma_{\mathrm{i}}$ in the semi-relativistic regime, from around 2.5 to 4 or 4.5 as $\sigma_{\mathrm{i}}$ goes from 0.1 to 10 . The authors emphasize that it is not yet clear, whether the computed power-law indices and cut-off energies are truly independent of the simulation length $L$ of the current sheet. In the ultra-relativistic regime, $\sigma_{i}>10$, the ion spectra show a power law which closely matches that of the electrons. For lower $\sigma_{i}$, the situation is somehow puzzling (see Fig. 26, upper row). A possible power law only appears at high ion energies while at lower energies the spectrum is rather flat and much harder, with a slope of about 1. This part is neither a clear power law nor, as it is much broader, a Maxwellian. This flat region in the spectrum turns downward significantly below the electron cut-off energy. However, at these energies, there are always more ions than electrons. The authors find no explanation for this behavior but speculate that indeed the power laws may show a break at energies where protons become trans-relativistic $\left(\omega \approx m_{\mathrm{i}} c^{2} \approx 103 \mathrm{MeV}\right)$. We add that yet another possibility is different dominant acceleration process for protons depending on energy.

Ball et al. (2018) present another study of collisionless relativistic REC of an electron-ion plasma, adding new facets to the picture (see Fig. 27). Firstly, the dependence on the $\beta_{\mathrm{i}}$ parameter is systematically explored, in addition to the dependence on $\sigma_{\mathrm{i}}$. They explore $\sigma_{\mathrm{i}}=0.1,0.3,1$ and 3 , while $\beta_{\mathrm{p}, \mathrm{i}}$ varies from $\beta_{\mathrm{p}, \mathrm{i}}=\beta_{\mathrm{p}, \mathrm{e}}=10^{-4}$ up to the maximum possible value of $\beta_{\max } \approx 1 / 4 \sigma_{\mathrm{i}}$. A realistic mass ratio is used. The computational domains are larger by at least a factor 5 than those of previous studies. The initial current sheet width is $\delta=80 c / \omega_{\mathrm{p}, \mathrm{e}}$. Periodic boundaries are used normal to the sheet. A moving injector and a dynamically-enlarging box is used. A description of the implementation of this boundary type is given in Sironi and Spitkovsky (2009). In this way, the magnetic flux is not limited to the one present at initial times. Finally, they trigger the start of REC by removing the pressure at one point within the sheet's center. Two exhausts with powerful depolarization fronts develop. As the boundaries are periodic normal to the sheet, the fronts meet to form a large plasmoid. When the fronts first meet, a secondary current sheet is created normal to the first sheet. In addition, they perform a study with different initial and boundary conditions, see below and Fig. 27, lower panels. All runs of this study are integrated till $2 t_{\mathrm{A}}$, independently of the box size and value of other relevant parameters, with $t_{\mathrm{A}}$ is given by the ratio between the length of the sheet and the Alfvén speed of the inflow, $t_{\mathrm{A}}=L_{\mathrm{x}} / U_{\mathrm{A}}$.

The initial current sheet fragments to a certain degree into secondary plasmoids and secondary X-points. For fixed $\sigma_{\mathrm{i}}$, the fragmentation is less pronounced for higher $\beta_{\mathrm{p}, \mathrm{i}}$ and, for fixed $\beta_{\mathrm{p}, \mathrm{i}}$, is more pronounced for higher $\sigma_{\mathrm{i}}$. Sironi et al. (2016) found the same dependences for the ultra-relativistic case.

Ball et al. (2018) find that the electron spectrum in the reconnection region is nonthermal and can be modeled as a power-law with slope $p$ which depends on $\sigma_{i}$ and $\beta_{\mathrm{p}, \mathrm{i}}$, as

$$
\begin{gathered}
p\left(\sigma_{\mathrm{i}}, \beta_{\mathrm{p}, \mathrm{i}}\right)=A_{\mathrm{p}}+B_{\mathrm{p}} \tanh \left(C_{\mathrm{p}} \beta_{\mathrm{p}, \mathrm{i}}\right) \\
A_{\mathrm{p}}=1.8+0.7 / \sqrt{\sigma}, \quad B_{\mathrm{p}}=3.7 \sigma_{\mathrm{i}}^{-0.19} ; \quad C_{\mathrm{p}}=23.4 \sigma_{\mathrm{i}}^{0.26}
\end{gathered}
$$


Thus, at low $\beta_{\mathrm{p}, \mathrm{i}}$, the slope is (nearly) independent of $\beta_{\mathrm{p}, \mathrm{i}}$ and hardens with increasing $\sigma_{\mathrm{i}}$, having a (nearly) equal form as found by Werner et al. (2018). At higher values of $\beta_{\mathrm{p}, \mathrm{i}}$, the electron power law steepens and the electron spectrum eventually approaches a Maxwellian distribution for all values of $\sigma$, see Fig. 27, upper left panel. At values of $\beta_{\mathrm{p}, \mathrm{i}}$ near $\beta_{\mathrm{p}, \mathrm{i} \text {,max }} \approx 1 / 4 \sigma_{\mathrm{i}}$, when both electrons and protons are relativistically hot prior to REC, the spectra of both species display an additional component at high energies, containing a few percent of particles, see Fig. 27, upper right panel.

Importantly, when using the same $\sigma_{\mathrm{i}}$ and $\beta_{\mathrm{p}, \mathrm{i}}$ as Werner et al. (2018), the nonthermal particle spectra found by Ball et al. (2018) are systematically softer than the one by Werner et al. (2018). It is shown in Ball et al. (2018) that this discrepancy may be caused by two reasons: firstly, the authors find numerically that a larger box size makes the spectra generally softer-though there are weak indications for a certain saturation of the slopes at the two biggest box-lengths they have used $\left(L_{\mathrm{x}} c / \omega_{\mathrm{p}, \mathrm{e}}=5^{\prime} 440\right.$ and $10^{\prime} 880$ ). Secondly, also found numerically, runs with a (single) triggered initial $\mathrm{X}$-point show a softer spectrum than runs in which the tearing instability produces spontaneously many X-points (as used by Melzani et al. 2014b and Werner et al. 2018). Indeed, Ball et al. (2018) reproduce exactly the same slopes as Werner et al. (2018) when using exactly the same setup and boundary conditions. The lower row of Fig. 27 summarizes the influence of the initial and boundary condition for the electron spectrum. Another finding is that protons have up to a magnitude larger mean energy than electrons, though protons show a steeper slope in their spectrum than electrons.

An important result is that for all low $\beta_{\mathrm{p}, \mathrm{i}}$, the time-evolution of non-thermal acceleration is different for electrons and protons. While electrons immediately develop a non-thermal tail in their spectrum, protons develop a non-thermal tail only after $t \approx 0.8 t_{\mathrm{A}}$, corresponding approximately to the time when the two reconnection fronts interact across the periodic boundaries. This may indicate another acceleration mechanism for the two species.

Result summary The last few years have brought progress in our understanding of the population of the non-thermal high-energy particles accelerated by relativistic REC. However, we emphasize again that all these results have been achieved on the basis of only one particular setting, 2D Harris or force free sheets. One should always keep in mind that this setting is a very particular one out of many other, probably more realistic settings. Under these conditions, the reconnection rate is given by Eq. (44). This rate is about 0.2 and thus higher than in the non-relativistic case. Two parameters are responsible for the formation of the power-law slope of the distribution of nonthermal particles accelerated in the reconnection event. The 'cold' magnetization $\sigma_{\mathrm{i}}$ and the magnetization $\beta_{\mathrm{p}, \mathrm{i}}$, which includes thermal aspects of the plasma in which the REC takes place. A pretty good expression for this power-law slope is given by Eq. (47). Note that for cold plasma (low $\beta_{\mathrm{p}, \mathrm{i}}$ ), this expression becomes fairly independent of $\beta_{\mathrm{p}, \mathrm{i}}$ and matches the simpler expression given by Eq. (46). Without a background field - unless in the highly relativistic limit - the ions gain more energy, up to $75 \%$ of the released magnetic energy in the semi-relativistic regime (see Eq. (45)).

There are, however, some aspects which disturb the picture and point to the need of additional work. Firstly, the exact shape of the power-law seems to depend, to a certain degree on the initial and boundary conditions (Fig. 27, lower row) and on the 
length of the Harris sheet. Secondly, if electrons and ions are hot before reconnection, there seems to exist an additional power-law slope in the electron spectra, at least at late times (Fig. 27, upper right panel). This may indicate that at least two different acceleration mechanisms are dominantly at work.

While the questions just addressed can be judged as minor, there remain two more fundamental largely open questions. One is the role of a background field (and such a background field is always present in a real environment). Melzani et al. (2014b) found indications that a background field (at least up to $B_{\mathrm{G}}=B_{0}$ ) does not affect the power-law slope of the electron distribution, but the spectrum evolves more slowly than without a background field (Fig. 25, right column panels). In addition, this study found that in the presence of a background field, electrons may gain more energy than protons, reversing the ratio found in simulations without a background field. However, one study is no study, the more as the low mass ratio used for these simulations may have spoiled the result. The second question is the exact shape of the proton population distribution. At low $\sigma_{\mathrm{i}}$, these spectra seem to consists of a very flat part at intermediate energies, before a clear power-law is established at higher energies (Fig. 26, upper left panel). This may again point to two different acceleration processes at work. The slope of the high-energy power-law for protons may be close to the one of electrons (Melzani et al. 2014b; Werner et al. 2018) or may be steeper (Ball et al. 2018):

\subsubsection{Selected physical processes which accelerate particles}

So far there is no comprehensive picture on which physical process is responsible for the acceleration of non-thermal particles in magnetic reconnection-though several reasonable ideas exist and some processes could be identified to be active, but possibly together with other processes. Therefore, at this place, we present those acceleration channels most discussed in the literature and the arguments of the authors who advocate them. We attempt a provisionally ranking in the next section.

Thermal exhausts As discussed in Sect. 2.5, REC at single X-points or on larger current-sheets produces exhausts where the particles leave the reconnection region (consult Fig. 10). Strictly speaking, the flow in the exhaust is not completely thermal. For instance, temperature is non-isotropic and electrons and ions do not have the same temperature, see e.g., Fig. 10 of Melzani et al. (2014a). However, the magnitude of the speed in the exhausts, $u_{\text {out }} \sim \sqrt{2} u_{\mathrm{A} \text {,in }}$ (Eq. 15), and the temperature in the exhausts can be understood on the basis of flow conservation laws between the inflow and the outflow.

Note that in a highly magnetized environment such as relativistic REC (but not exclusively), the Lorentz-factor of the exhausts may already be of order of a few, because the Alfvén speed of the inflow may already be very close to the speed of light.

Below, the dynamics of chains of X-points and current sheets are discussed. There, exhausts of the different sites collide and form plasmoids (Fig. 24). But in nature there are also reconnection sites found with just one X-point. There, the exhausts can freely expand to form jets. This mechanism is used to explain jets on all scales, from the solar atmosphere and corona (Zharkova et al. 2011), but also from compact objects (de Gouveia dal Pino and Lazarian 2005). The more, small reconnection events and 
associated exhaust within large scale jets may be at the origin of fast $\mathrm{TeV}$ variability in blazars (Giannios et al. 2009; Khiali et al. 2015).

Dynamics of plasmoids and chains of plasmoids We now look at a longer current sheet that break apart and, consequently, allows a variety of potential particle acceleration mechanisms - to be further detailed below-to act on the plasma. If the sheet is long enough, many X-points will develop, with associated exhausts. Exhausts of neighboring X-points collide and form plasmoids, plasma regions which are bound by a strong circularly closed magnetic field (consult Fig. 24). The formation of plasmoids ( $\rightarrow$ Colliding plasmoids) has the potential to accelerate particles as the border of two associated exhausts, called dipolarization fronts, form approaching magnetic mirrors, see e.g. Lapenta et al. (2015).

The field within the plasmoid tends to zero. Thermal plasma within the plasmoids cannot escape as the strong encircling field deflects particles immediately back to the interior. As REC goes on and more of the inflowing plasma is being processed, islands potentially grow in size and their encircling fields grow in strength. Plasmoids will also merge and grow in size. After merging, plasmoids will contract and give rise to an important acceleration mechanism ( $\rightarrow$ Contracting plasmoid). The process of breaking a current sheet will also generate turbulence, another important source of particle acceleration ( $\rightarrow$ Turbulence).

At this place, the stability of current sheets, the formation and dynamics of plasmoids cannot be reviewed in detail. This process sets the stage for the acceleration of ultra-fast particles, but not directly causes it. For a deeper understanding of the process, the reader may consult the vast literature on the subject, e.g. Loureiro et al. (2007), Lapenta (2008), Samtaney et al. (2009), Lapenta and Lazarian (2012), Kowal et al. (2012), Markidis et al. (2013), Kagan et al. (2013), Loureiro and Uzdensky (2016), Sironi et al. (2016), Kowal et al. (2017).

Contracting plasmoids PIC simulations show that contracting plasmoids (see structure B in Fig. 24) can efficiently accelerate electrons (Drake et al. 2006) and ions (Drake et al. 2010) to super-Alfvénic speeds by a Fermi-like process. Such particles are injected into magnetic islands from the exhausts of $\mathrm{X}$-points of a reconnecting current sheet. Particles approaching the strong magnetic fields encircling magnetic islands will be turned around by these fields and are captured in this way within the island, bouncing in the island backwards and forwards or move along the magnetic field lines. When islands are freshly formed, the encircling field also lets contract the island, up to a point where a quasi-stationary equilibrium is reached between the pressure of the enclosed particles and the tension force of the magnetic structure. In this way, the particle will gain energy at each bounce. When more energized, the particles will start to diffuse out of the island and either escape or, in a more complex situation, may be kept by another island held together with a stronger field.

Counting on this mechanism, Drake et al. (2010) can explain the anomalous cosmic ray (ACR) energy spectrum observed by both voyager missions in the region between the solar wind termination shock and the heliopause (note that the same idea was also developed in Lazarian and Opher 2009). Drake et al. (2010) firstly perform large scale MHD simulations of the solar wind which show stripes of inversed field directions 
due to the non-alignment of the magnetic dipole of the Sun with its rotation axis. Current sheets develop in the region where the field polarity changes. (This is very similar to the striped neutron star winds (Kirk 2004; Uzdensky et al. 2011; Cerutti et al. 2012a, 2014a; Bühler and Blandford 2014).) The MHD simulations show that stripes and current sheets are compressed in the passage of the solar wind through the solar wind termination shock, setting the stage for REC in the now unstable sheets. The post-shock situation can be well approximated by multi-layered Harris-sheets.

Subsequent PIC simulations using the plasma-parameters found in the MHD simulations demonstrate that particles can be accelerated to several tens Alfvénic speeds, forming a power law with a spectral index of about 1.5. The multi-layered Harrissheets break off, forming a network of plasmoids which collide and collapse. By a thorough analysis of the data, Drake et al. (2010) show the outstanding role contracting islands play in the acceleration process.

The model assumes that interstellar ions are pickuped by the solar wind and advected subsequently through the solar wind termination shock. Thus the model can also explain similarities in the spectra of different ion species observed by the voyager missions. The result achieved demonstrates the power of combining large-scale MHD simulations with detailed kinetic simulations.

Subsequently, Kowal et al. (2011) performed MHD simulations in two and three space dimensions of the same multi-layered Harris sheets. A dynamic network of magnetic islands develops, similar to the one observed in the PIC simulations of Drake et al. (2010). Test-particles are injected into this configuration and integrated with the sixth-order implicit Runge-Kutta-Gauss method (Sanz-Serna and Calvo 1994) which conserves particle energy and momentum. The accelerated test-particles show also a super-Alfvénic distribution though there are differences in the distribution as compared to Drake et al. (2010). Contracting island again play a dominant role in the acceleration process. But the authors report also on drift acceleration along the magnetic field discontinuity and the Fermi-process between the converging inflows (see the corresponding paragraphs within this section). The reasons behind these differences remain open. The paper extends the multi-layered Harris sheet configuration to three space dimensions and repeats the analysis. They find substantial differences in the acceleration process and stress the need for more comparative studies between $2 \mathrm{D}$ and $3 \mathrm{D}$ settings.

Montag et al. (2017) develop a general framework which includes compressibility and non-uniform fields to analyze the process of contracting plasmoids. They derive an expression for the power-law scaling of the distribution function and for the rate at which the power-law develops in time. In analogy to the case of acceleration by the converging inflows, the spectrum gets harder if the compression increases, which is generally true for Fermi-like processes. The authors also find that a guide field of order unity suppresses the development of power-law distributions.

Colliding plasmoids As described, plasmoids eventually collide. Subsequently, they first grow and then contract, thereby accelerating particles.

There are two other interesting points in colliding plasmoids. Firstly, at the contact interface between the coalescing plasmoids secondary current-sheets with secondary REC develop, mostly normal to the primary sheet. This secondary REC may support or 
suppress particle acceleration, its effect seems, however, to be small, see the discussion in Bessho and Bhattacharjee (2012). Secondly, even before collision, in the phase they approach each other, a Fermi-like acceleration process may work as particles are reflected on the plasmoids and travel back and forth between neighboring approaching plasmoids (Lapenta et al. 2015, for instance).

Werner et al. (2018) use this process to explain the expression for the power-law index they found (Eq. 45). The acceleration by approaching plasmoids is a second order Fermi process as the movement of the plasmoids can be regarded as stochastic. The energy gain per bounce is thus $\Delta \epsilon \simeq\left(u_{\mathrm{pl}} / c\right)^{2} \epsilon$, where $\epsilon$ is the particle energy without the rest-mass energy and $u_{\mathrm{pl}}$ the speed of the plasmoid. The typical bounce time can be approximated by $t_{\mathrm{b}} \sim \lambda_{\mathrm{pl}} / c$. Thus, the acceleration time-scale is $t_{\mathrm{acc}} \equiv \epsilon \Delta t_{\mathrm{b}} / \Delta \epsilon=$ const $\cdot c \lambda_{\mathrm{pl}} / u_{\mathrm{pl}}^{2}$. The process ends when the two plasmoids collide and the particle escapes, at $t_{e s c} \simeq \lambda_{p l} / u_{p l}$, where $\lambda_{p l}$ denotes a typical distance between plasmoids. By this, the Fermi II power law index, $p$, can be written as $p=1+t_{\text {acc }} / t_{\text {esc }}=$ $1+$ const $c / u_{\mathrm{pl}}$. Werner et al. (2018) assume that $u_{\mathrm{pl}} \simeq U_{A}$. In the regime where $\sigma_{\mathrm{i}}<<1$ (but still $\sigma_{\mathrm{e}}>>1$ ), $U_{\mathrm{A}} \approx c \sigma_{\mathrm{i}}^{1 / 2}$, and thus $p=1+C \sigma_{\mathrm{i}}^{-1 / 2}$, which has the form of Eq. (45). We note that one can repeat the exercise for any other Fermi II process taking place within the sheet, in particular also for acceleration due to turbulence. Ball et al. (2018) account for a variant of this process to explain the additional component in the power-law at high energies when the inflow is hot $\left(\beta_{\mathrm{p}, \mathrm{i}}\right.$ close to $\left.\beta_{\mathrm{p}, \mathrm{i}, \mathrm{max}}\right)$.

Turbulence As discussed in Sect. 2.5, the break apart of current sheets by tearing mode instabilities (or a combination of tearing and kink mode in 3 spatial dimensions) generates turbulence in the region where REC takes place. This turbulence, in combination with turbulence in the inflows, is responsible that collisional REC becomes as fast as observed (Kowal et al. 2009; Lapenta and Lazarian 2012). In the frame of test particles, it was shown that MHD fast and slow compressible modes accelerate energetic particles through the second order Fermi acceleration. Density fluctuations in converging flows can enable first order Fermi acceleration of particles (Kowal et al. 2012). Kowal et al. (2017) provide a thorough analysis of the statistics of the reconnection-driven MHD turbulence. Lazarian et al. (2012) and Lazarian et al. (2016) review the development and the character of (self-generated) turbulence related to magnetic reconnection, in the classic as well as in the relativistic regime.

Corresponding studies for the collisionless case only recently start to appear. Zhdankin et al. (2018) study forced turbulence using PIC simulations. They show that particles can indeed be accelerated to very high energy also in the case of collisionless turbulence. Figure 28, top panel, shows the time-evolution of the spectrum of accelerated particles, converging to a power law of the form $\gamma^{-3}$. The lower panel shows the dependence of the domain resolution, from $512^{3}$ to $1536^{3}$ cubed.

In a preprint, Zhdankin et al. (2019) present corresponding results for an ionelectron plasma and come to the conclusion that non-thermal acceleration is efficient for both species in the fully relativistic regime. In the semi-relativistic regime, while still efficient for cosmic ray production, the mechanism is less efficient for electrons. For the production of hard non-thermal radiative signatures a (very) low $\beta_{\mathrm{p}, \mathrm{i}}$ is necessary, or nearly relativistic ion-temperatures. The authors emphasize that the result is still somehow preliminary as it is not yet established how this result scales to a large 

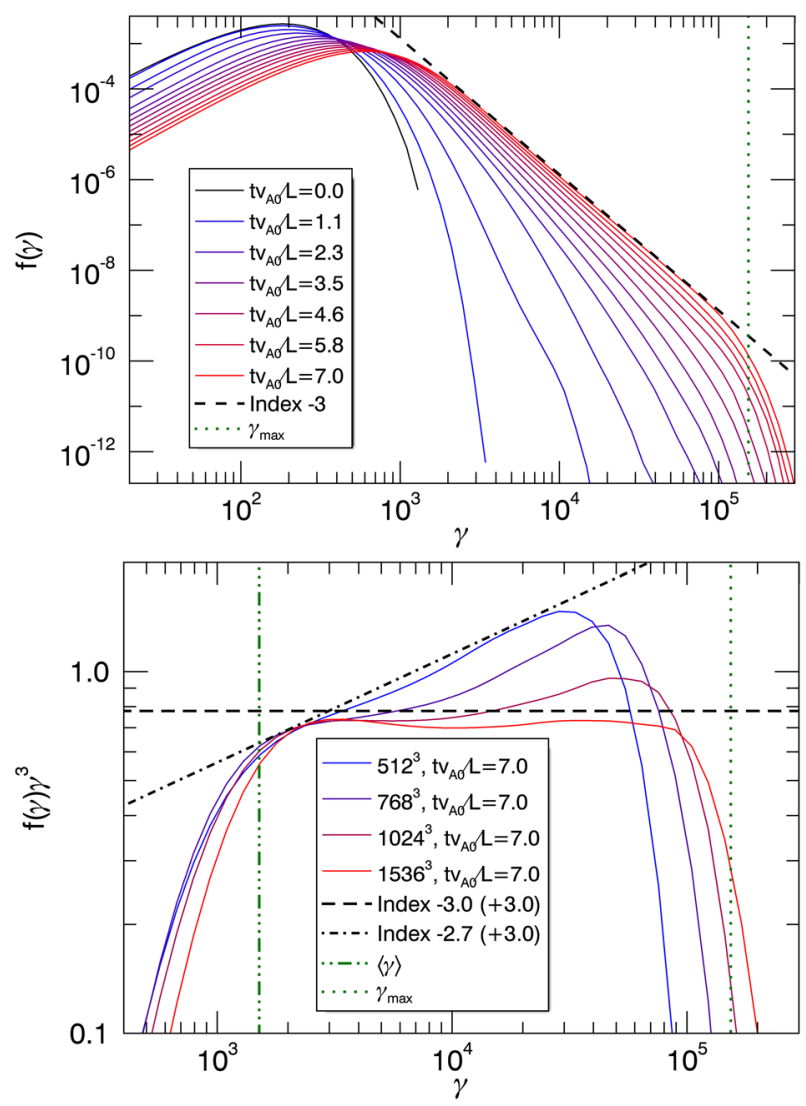

Fig. 28 Top panel: evolution of the particle Lorentz factor (energy) distribution $f(\gamma)$ for the $1536^{3} \operatorname{sim}^{-}$ ulation of forced collisionless turbulence. The dotted line indicates the slope expected for a second order Fermi-process. Bottom panel: compensated distribution $f(\gamma) \gamma^{3}$, at fixed time $t U_{A_{0}} / L=7.0$, for varying system sizes. Power laws with pre-compensated index -3.0 (black dashed) and -2.7 (black dash-dotted) are also shown, along with the mean energy $\langle\gamma\rangle$ (green dash-dotted) and system-size cutoff $\gamma_{\max }$ (green dotted) for the $1536^{3}$ case. Images reproduced with permission from Zhdankin et al. (2018), copyright by AAS

system size. Related to these simulations, the authors have statistically analyzed collisionless turbulence (Zhdankin et al. 2017) and make a connection to the Fokker-Planck framework (Wong et al. 2019).

A word of caution, equally raised by the authors, must be added. The forcing of the turbulence implies a steady energy input into the system. As no energy sinks are present, energy may pile up to give artificial heating and non-thermal particle acceleration. More studies are clearly needed. Also, non-global compression was present which would rise the value for the power-law index (Bell 1978a; Drury 2012).

Nevertheless, it can be firmly stated that turbulence is present in reconnection sites, both, collisional and non-collisional, and that this turbulence can contribute to particle acceleration. We note these results are also very promising to advance our understanding of particle acceleration in shocks. 
The reconnecting electric field This field is an important source of particle energization in the relativistic case.

As particles are de-magnetized in the current sheet, this field can directly accelerate particles without perturbation by other structures. However, the width of the region where the electric field is strong is not too large, i.e., it is given by $E>c B$, or with a guide field, by $\mathbf{E} \cdot \mathbf{B} \neq 0$.

In non-relativistic REC, this process cannot be too effective as the electric field is too weak as compared to the width of the acceleration zone and the particles escape soon (Drake et al. 2010; Kowal et al. 2011; Drury 2012).

In the relativistic case, however, the process becomes important and the accelerated particles will form a power law. Particles enter the region with a large electric field at all distances from the central X-points. The particles close to the X-point will only slowly be turned, by $B_{X}$, towards the exhausts, in contrast to those particles which enter the sheet at some distance from the X-point. The longer the particle is held in the central region, the longer it can be accelerated by the reconnection electric field. As emphasized by Zenitani and Hoshino (2001) the gyro-radius of relativistic particles grows with their Lorentz-factors. Already accelerated particles thus stay even longer within the region of a large electric field and hard tails of the distribution can grow. These authors predict, for a pair plasma, a power-law distribution of non-thermal particles created by the reconnection electric field of $p \propto c B_{x} / E_{R e c}$ Bessho and Bhattacharjee (2012) refined the analysis in two aspects, also for a pair plasma. Firstly, they looked closer at the creation of the power law distribution but then included also the process when the particles finally have to leave the region immediately around the $\mathrm{X}$-point. The latter produces an exponential cut-off such that the particle distribution function can be written in the form

$$
f(\gamma) \sim \gamma^{-1 / 4} \exp -a \sqrt{\gamma}
$$

with $a$ a constant of order $c B_{x} / E_{\mathrm{Rec}}$. Note that this is a very hard distribution. The authors emphasize that particles accelerated by the reconnection electric field may be further accelerated by other processes, in the field when they swirl around contracting islands or when they encounter other X-points. We add that any other of the processes described in this section could be tapped. PIC simulations of relativistic collisionless REC confirm that this process significantly contributes to the high-energy spectrum, for both cases, of a electron/positron pair plasma (Bessho and Bhattacharjee 2012; Sironi and Spitkovsky 2014) and an ion-electron (Melzani et al. 2014b; Ball et al. 2018).

Fermi process within the inflow region Recall that the inverse field components float, due to $\mathrm{E} \times \mathrm{B}$-drift, towards the region where they will reconnect (Fig. 1, right panel).

de Gouveia dal Pino and Lazarian (2005) point out that this may, in principle, accelerate particles by a first order Fermi-process (see Sect. 2.2.1), if particles are allowed to cross the reconnection region and to be ping-ponged between the lower to the upper inflow into the sheet. Repeating the argumentation given by Bell (1978b) for 
the shock case, de Gouveia dal Pino and Lazarian (2005) derive an emerging spectrum of accelerated particles of $N(E) \propto E^{-5 / 2}$ for this acceleration mechanism.

An important point is raised by Giannios (2010) who states that this mechanism does not depend on the not well known reconnection mechanism but works solely on the converging inflows to the reconnection region. Be $\delta$ the width of the dissipation region and $R_{\mathrm{G}}$ the gyro-radius associated to the undisturbed magnetic field and $\theta$ the angle under which the particle enters the reconnection layer. Be this angle moderately small (as compared to the length of the sheet, $L$ ) and consider the case where the particle can freely pass the reconnection region. Then, the particle will gyrate around the field drifting into the reconnection site and will cross back the sheet to its other side, where it gyrates back and forth. Of course, turbulent field fluctuations or collisions may scatter the particle as well, but their presence is not really necessary. In addition, the particle is gaining energy (though less) at each passage in the reconnection electric field. Giannios (2010) refers to this process as the betatron effect.

The energy amplification factor, A, for one cycle can then be derived to:

$$
A(\theta)=\Gamma_{\mathrm{r}}^{2}\left(1+\beta_{\mathrm{r}} \cos \theta\right)^{2}
$$

where $\theta$ denotes the angle at which the particle enters the reconnection layer and $\Gamma_{\mathrm{r}}, \beta_{\mathrm{r}}$ the Lorentz-factor and normalized speed of the inflow. We note that with $\theta=0$ and $\beta_{\mathrm{r}} \ll 1$, the formula of de Gouveia dal Pino and Lazarian (2005) is recovered. The time-scale of the acceleration process is

$$
t_{A c c}=\frac{2 \pi \gamma m c^{2}}{(1-1 / A) e B c}
$$

and is thus of the order of the gyration period of the largest $\gamma_{\mathrm{e}}$ factor. Giannios (2010) shows that for an isotropic particle distribution (and thus $\theta$ ), the amplification amplitude, $A$, is only about one quarter smaller as for the case $\theta=0$.

The process is limited by two factors, the size of the current sheet and by radiative losses. These are dominated by synchrotron losses while photo-pion production turn out to be much less important (Waxman 1995). Assuming jets carrying field-reversed components of length of the jet diameter, Giannios (2010) estimates that protons can reach energies up to $10^{20} \mathrm{eV}$ in GRBs or luminous AGN jets. For iron, an important species in very high energy cosmic rays, the same limit applies. For electrons, however, the acceleration process stops much earlier due to synchrotron losses. The author emphasizes that an important pre-requisite for the process to work is that it can only be initiated if the particles are sufficiently pre-accelerated, because, otherwise, the gyro-radius of the particles is not large enough to let the particle stream over the reconnection region.

Drury (2012) points out that the spectrum derived by de Gouveia dal Pino and Lazarian (2005) is probably much too weak (i.e., the power-law slope should be much smaller than 5/2). This, because the compression of the plasma within the sheet is not considered. The paper provides arguments that, in the case of REC, the compression is probably larger than in the shock case, where mostly a compression ratio of 4 is assumed, which corresponds to an adiabatic shock. For REC, spectra like $f(p) \propto p^{-2}$ 
or $N(E) \propto E^{-1}$ have to be expected. This was the first indication that spectra of particles accelerated in magnetic reconnection events may indeed be very hard, much harder than for particles accelerated by the Fermi process in shocks.

Bosch-Ramon (2012), considering compressible effects, realistic cross B-field diffusion coefficients, and accounting for synchrotron cooling, shows that for protons a maximum energy of

$$
E_{\max } \approx 60(\chi / k)^{1 / 2}(v / c)^{1 / 2} \frac{1}{\sqrt{B}_{0}} \mathrm{TeV} .
$$

can be reached. $\mathrm{k}$ and $\chi$ depend on the concrete microphysics, with good arguments for $\chi / k \approx 1 / 10$. The spectrum of the accelerated particles is harder than standard Fermi I.

Provornikova et al. (2016) analyze possible compression rates of REC in the solar corona. They look at configurations such as a Harris current sheet, a force-free current sheet, and two merging flux ropes. Plasma parameters are taken to be characteristic of the solar corona. It is found that plasma compression is expected to be strongest in lowbeta plasma $\beta_{\mathrm{p}} \sim 0.01-0.07$ at reconnection magnetic nulls and can be as high as a factor 10. Provornikova et al. (2018) derive magnetic wave solutions in compressible reconnection sites and claim that such waves may act as scattering centers for the Drury-mechanism.

In conclusion one may say that this is a promising process whenever there are pre-accelerated particles present which can initiate the back- and forth-bouncing.

Drift acceleration If the particles enter the diffusion region or if they leave the current sheet the magnetic field changes substantially. In this situation, the particles encounter magnetic drifts and magnetic drift acceleration (see Sect. 2.3 for the description of the equivalent mechanism in shocks). Kowal et al. (2009) and Guo et al. (2016) report that this mechanism contributes to the acceleration of particles, both in the collisional (Kowal et al. 2009) and non-collisional (Guo et al. 2016) case.

Associated shocks de Gouveia dal Pino and Lazarian (2005) point to another interesting possibility: analogous to the sun, eruptive reconnection may push matter away and produce a shock-wave in which particles can be accelerated by the 'ordinary' Fermi-mechanism related to shocks, leading to a power-law slope of 2 .

Dimensionality The role of dimensionality in the particle acceleration process is largely unexplored.

2D spatial setups can easily be extended to 3D spatial setups by just expanding the $2 \mathrm{D}$ configuration to the third dimension in a planar way. Such configurations will still be subject of the tearing mode, but, in addition, the 3D extension will be subject to the kink instability. Cerutti et al. (2014b), using PIC simulations with radiative feedback, compare the cases where either the kink or the tearing instability grows faster and discuss application to the Crab nebula. In their setup they found the kink mode to dominate, leading to a disruption of the current sheet and associated turbulence unless the kink-mode is stabilized by a background magnetic field. The same result is also 
found by Oishi et al. (2015) in 3D MHD simulations. Other authors, using different configurations, found that rather the kink mode develops slower than the tearing mode even without a guide field. Here, REC develops essentially similarly as in two spatial dimension. Oblique modes, a combination of tearing and kink modes, are possible and may even grow fastest (Daughton et al. 2011).

The results of different 3D kinetic studies of current-sheet reconnection do not yet converge to a unique picture. Some authors claim that there are small differences in the reconnection rate between 2D and 3D, e.g., (Liu et al. 2012; Daughton et al. 2014, for non-relativistic REC) and (Guo et al. 2014a, for the relativistic case). On the other hand Sironi and Spitkovsky (2014) found a four times lower rate for 3D REC of a relativistic pair plasma as compared to $2 \mathrm{D}$ REC.

In a series of papers it was advocated that the drift kink instability can modify the electric and magnetic field structures in an anti-parallel reconnection layer and prohibit non-thermal acceleration (Zenitani and Hoshino 2005a, b, 2007, 2008). But other authors found that the kink mode cannot suppress the acceleration of particles (Liu et al. 2011; Sironi and Spitkovsky 2014).

There are very few generic 3D configurations which include 3D nulls. Based on observations, Baumann and Nordlund (2012) reconstruct a field configuration within the solar corona and use it as initial condition for 3D PIC simulations. Olshevsky et al. (2013) simulate REC, starting from a cluster of eight null points. Much more work will be necessary in future to get a more comprehensive picture of $3 \mathrm{D}$ reconnection events.

\subsubsection{A critical discussion and outlook}

The first point to state is that there has been a tremendous progress in our understanding of magnetic reconnection in the last few years. Given a correct environment, both collisional and non-collisional magnetic reconnection proof to be fast. For the collisional case, both, self-generated and/or external turbulence is the key-ingredient to make the process fast. The collisionless case turns out to be always fast. With this progress, we now can understand qualitatively, and even to a good degree quantitatively the 'thermal aspects' of REC.

This review has for subject the non-thermal, ultra-energetic particles. Mostly PICsimulations have shown that such particles can originate from reconnection sites. Many questions remain, however. We want to address three of them, A-C, in the following paragraphs.

(A) Open questions concerning small scale kinetic simulations As summarized at the end of Sect. 4.2.2, we have, on the basis of 2D current sheet simulations some definitive results. However, and as emphasized also there, many details remain unanswered also for this case. There remains, in our view, three large questions which need to be answered before we can state that our understanding even for this simple case is sufficiently secured.

1. There is only one study which includes a guide field (at least for ion/electrons). This study has brought interesting results, the independence of the spectral slope on 
the presence and the strength of a guide field and that the energy partition between electrons and ions may critically depend on the presence of a guide field. Guide fields are necessarily present in a current sheet like reconnection event in space. Corresponding large parameter studies, which derive the relations for ion-electron energy partition, the dependence of the power-law slope on the magnetization and the thermal state of the inflow when a guide field is present are urgently needed.

2. The situation in three spatial dimensions remains unclear, even for the most simple case, the extension of a $2 \mathrm{D}$ current sheet towards the third dimension and a comprehensive study is lacking.

Beyond this simple case, there are much more complicated reconnection topologies present in 3D than the simple extension of a $2 \mathrm{D}$ current sheet to 3D. An overview of such topologies can be found in Birn and Priest (2007, Chapter 2) and Pontin (2011). None of these topologies have been systematically addressed by kinetic simulations.

3. Finally, REC is always accompanied with radiative emission. In a magnetized environment, synchrotron radiation is always present and cool the particles which emit. In addition, these photons undergo inverse Compton scattering with the hot particles, cooling them again. Many reconnection sites are embedded in a strong external cold radiative source, e.g., companion stars in X-ray binaries, leading to additional cooling. Radiative effects introduce new parameters into REC not discussed here so far. For instance, REC in a micro-quasar corona close to the hole and in the $\gamma$-ray emitting region of an extragalactic jet takes place with the same magnetizations (see Table 3). Without considering radiative effects, REC in both environments features the same reconnection rate, particle spectra, or energy distribution. However, the field strength differs by six orders of magnitude and thus the effect of synchrotron radiation is largely different.

There are first attempts to account for radiative emission in PIC-simulations. Werner et al. (2018) perform a relativistic 2D Harris sheet study of a pair plasma and describe effects of external inverse Compton cooling on the basic dynamics, the non-thermal particle acceleration, and radiative signatures. They find the reconnection rate and the overall dynamics basically unchanged. Important differences are found for the particle spectra. They still show a hard power law (index $\geq-2$ ) as in nonradiative REC, but transition to a steeper power law that extends to a cooling-dependent cutoff. The steep power-law index fluctuates in time between roughly -3 and -5 . Some other studies which include radiative losses address mostly REC in pulsar winds (Cerutti et al. 2014b, a, 2016). Also the community of the laser-plasma facilities starts to study radiative effects with PIC codes as newer, more powerful lasers establishes a regime where radiative losses essentially co-determine the dynamics, see for instance Wallin et al. (2015), Gonoskov and Marklund (2018), Blackburn and Marklund (2018).

(B) How are particles accelerated? So far, it is not possible to rank the relative importance of the different acceleration processes discussed in Sect. 4.2.3. It seems likely that different mechanisms are at work even for a simple setting, but the more if one also considers the prevailing large scale physical conditions, e.g., hot non-relativistic pair plasma or cold ultra-relativistic electron proton plasma (see also part C below). 
Most papers mentioned above come to this conclusion. In the same direction points the finding that the distribution functions of the non-thermal particles, electrons and protons, often show broken power-law slopes, with up to three different slopes and an exponential cut-off. Even if some studies suggest that the shape of the distribution functions for protons and electrons are close to identical, other studies shows differences between the shapes of electron and proton distribution functions. In relativistic REC, the direct acceleration by the reconnection electric field is unambiguously identified as one important ingredient - in contrast to the non-relativistic case. However, all studies showed that it is accompanied by some stochastic Fermi-process. Whether this process operates between magnetic islands or other magnetic structures or is just a consequence of kinetic turbulence in the diffusion region and in the current sheet, is not yet clear.

First order Fermi processes can also be present. Such a process certainly works in contracting islands and seems to accelerate the population of particles which is originally located in the current sheet. If particles can cross island-boundaries, this process may contribute to the acceleration of other particles as well. Whether, or under what physical conditions, the first order Fermi process between the converging inflows can work is an open question. In none of the relativistic simulations it has been observed. The reason may be that a Harris sheet is a too symmetric constellation where it is very hard to initiate particle motions normal to the direction of the sheets which are sufficiently fast to move the particle out of the diffusion region. However, if one would be able to find a mechanism to pre-accelerate particles, this mechanism could become operational. Corrugated sheets, spine-fan or other topologies may support the initialization of this mechanism. This question remains open and is part of the next point of discussion.

(C) Magnetic reconnection in a large scale environment and in real objects Understanding microphysical processes is decisively important but, on the other hand, only one part of the game to understand REC and to what degree REC contributes to the emission of real objects via both, the leptonic and hadronic channel. And it does not answer the question whether REC contributes to the cosmic ray flux. To get the answers to such questions, we definitely need to combine microphysics with large scale flow.

It is important to understand what triggers a reconnection event and the nature of the event. Are, in real astrophysical objects, quasi-stationary configurations present, like Harris-sheets or other magnetic nulls which eventually get unstable? Or are reconnection events driven by large-scale flows more important. Related, does REC start at one point or will a network of reconnection points establish? All this is largely unexplored.

We therefore advocate simulations of large scale MHD flows, entire accretion disks and large portions of jets in high-energy objects. Such MHD simulations must control magnetic diffusion, to be able to identify reconnection sites and topologies. Ideally, such large scale simulations are also resistive. Only on the basis of such MHD simulations we will obtain a good estimate of the spatial and temporal distribution of reconnection events in such objects. Subsequent microphysical studies which adapt the configurations found by the MHD solutions will then allow to model photon and possible neutrino emission. MHD simulations of magnetic reconnection will be reviewed in a future version of the review. 


\section{Macro scale numerical particle acceleration studies}

At scales comparable with the system size dynamics are often treated in the magnetohydrodynamics (MHD) approximation. In this approximation different methods have been developed to handle the acceleration and propagation of energetic supra-thermal particles, which are listed and described below. Before treating this aspect we present in Sects. 5.1 and 5.2 the main MHD solvers used in most of modern codes. A discussion concerning relativistic MHD is also included. Detailed monographs and reviews on the subject can be found, e.g., in Leveque (1998), Martí and Müller (2015) and references therein. The next sections treat the way CRs can be coupled with MHD. Section 5.3 discusses the multi-fluid approach where energetic particles are treated as a fluid. Section 5.4 describes the procedure to combine kinetic and HD/MHD methods, in particular the way to treat energetic particles back-reaction over fluid solutions (see Sect. 3.6). Section 5.5 presents the P(MHD)IC method in some details, this method combines simulation techniques exposed in Sect. 3.3 to investigate microscale physics but uses the electromagnetic field derived from the MHD equations. Section 5.7 discusses semi-analytical calculations of the problem of DSA, introduced in Sect. 2.2.

\subsection{The equations of magnetohydrodynamics}

The use of a magnetohydrodynamic (MHD) approach is crucial for the description of the large scale astrophysical phenomena involving collisional plasma, i.e. if the timescale associated to collision is shorter than the system dynamical time. This circumstance can occur for instance in supernova remnants, stellar bubbles and accretion discs. However, most of astrophysical shocks (including SNR shocks) are non-collisional as previously explained and their structure can not be dynamically described using a MHD model. However, if collective interactions are sufficiently frequent to keep the system isotropic, if electroneutrality can be assumed (which is the case for scales larger than the Debye length), in the cold plasma approximation the MHD equations reduce to the one-fluid system of equation derived in Eq. 52 below. This system describes long wavelength and low frequency perturbations.

\subsubsection{Classical magnetohydrodynamics}

MHD equations couple fluid mechanics equations and Maxwell's equations. They are obtained by averaging the moments of the Boltzmann equation over the velocity space. Particle density conservation is deduced by taking the zeroth order moment of the Boltzmann equation, the momentum conservation equation is obtained by taking the first order moment, and the energy conservation equation is obtained by taking the second order moment. Each of these moment equations introduces a new unknown function: the continuity equation introduces the velocity, the moment conservation equation introduces the pressure and the energy conservation equation introduces the internal energy. Additional assumptions are then required to close the system. To that aim, an equation of state is usually introduced which expresses the internal energy as function of density and pressure, but other assumptions can be used concerning energy fluxes. 
The full MHD equations for a single fluid are given in a conservative form as follows:

$$
\begin{aligned}
\partial_{t} \rho+\nabla \cdot(\rho \mathbf{u}) & =0, \\
\partial_{t}(\rho \mathbf{u})+\nabla \cdot\left(\rho \mathbf{u u}-\mathbf{B B}+\left(p+\frac{B^{2}}{2}\right) \mathbf{I}\right) & =\mathbf{0}, \\
\partial_{t}(e)+\nabla \cdot\left(\left(e+p+\frac{B^{2}}{2}\right) \mathbf{u}-(\mathbf{B} \cdot \mathbf{u}) \mathbf{B}\right) & =0, \\
\partial_{t}(\mathbf{B})-\nabla \times(\mathbf{u} \times \mathbf{B}) & =\mathbf{0},
\end{aligned}
$$

where $\rho$ is proper rest mass density, $p$ is the thermal pressure, $\mathbf{u}$ is the Eulerian fluid velocity vector, $\mathbf{B}$ is the magnetic field, and $e=\frac{p}{\gamma_{\mathrm{ad}}-1}+\frac{\rho u^{2}}{2}+\frac{B^{2}}{2}$ is the total energy density, where $\gamma_{\text {ad }}$ is the gas adiabatic index. The ideal Ohm's law (perfect conductivity) is retained: $\mathbf{E}=-\mathbf{u} / c \times \mathbf{B}$.

These equations are justified for a plasma where the relevant time scales are long in comparison with microscopic particle motion time scale and spatial scales are large in comparison with the thermal ion gyroradius and Debye length.

\subsubsection{Equation of state (EOS)}

The set of partial differential Eq. (52) governing compressible fluid dynamics is incomplete. There are more unknowns than equations and then an additional closure equation is required. Usually, this equation involves the internal energy, the thermal pressure, and the density, and in some cases the temperature as well. This closure equation describes the thermodynamical processes in the fluid. In a real plasma the different particle populations can have different thermodynamical behaviour. For simplicity, the most used closure equation is the polytropic equation of state,

$$
\frac{\mathrm{d}}{\mathrm{dt}}\left(\frac{p}{\rho^{\gamma \mathrm{ad}}}\right)=0
$$

in an adiabatic gas with one degree of freedom $\gamma_{\mathrm{ad}}=5 / 3$ and in an isothermal gas $\gamma_{\mathrm{ad}}=1$.

\subsection{Numerical solutions}

The finite volume method (FVM) is widely applied to solve problems described by hyperbolic partial differential equations (PDEs) as is the case for MHD equations. In this method the set of Eq. (52) is written in a shortened conservative form:

$$
\partial_{t} \mathbf{U}+\partial_{\mathrm{i}} \mathbf{F}^{\mathrm{i}}(\mathbf{U})=\mathbf{0},
$$

where $i=(x, y, z)$ are the space variables, the conserved variables are given by 


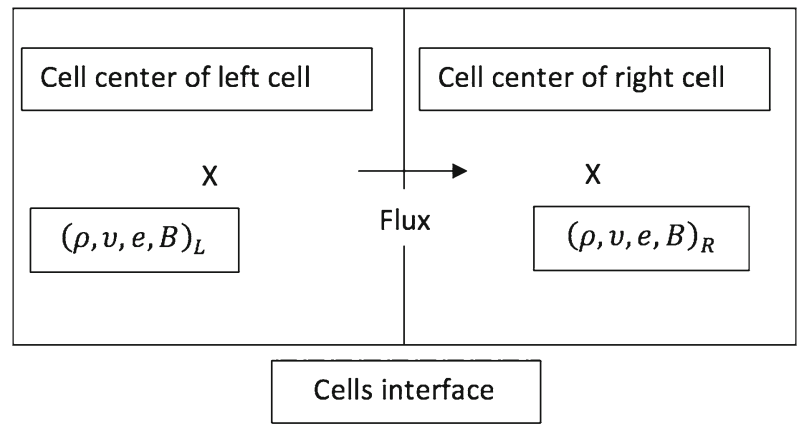

Fig. 29 Finite volume scheme with variables set at cell center and flux computed at face center

$$
\mathbf{U}=\left[\begin{array}{c}
\rho \\
\rho \mathbf{u} \\
e \\
\mathbf{B}
\end{array}\right]
$$

and the associate fluxes are

$$
\mathbf{F}=\left[\begin{array}{c}
\rho \mathbf{u} \\
\rho \mathbf{u} \mathbf{u}-\mathbf{B} \mathbf{B}+\left(p+\frac{B^{2}}{2}\right) \mathbf{I} \\
\left(e+p+\frac{B^{2}}{2}\right) \mathbf{u}-(\mathbf{B} \cdot \mathbf{u}) \mathbf{B} \\
\mathbf{u B}-\mathbf{B u}
\end{array}\right]
$$

Numerically Eq. (54) are discretized in space and time as

$$
\mathbf{U}^{\mathrm{n}+1}=\mathbf{U}^{\mathrm{n}}+\Delta t^{\mathrm{n}}\left[\sum_{\mathrm{idim}=1}^{\mathrm{idim}=\mathrm{ndim}} \frac{\mathbf{F}_{\mathrm{idim}}^{\mathrm{i}+\frac{1}{2}}-\mathbf{F}_{\mathrm{idim}}^{\mathrm{i}-\frac{1}{2}}}{\Delta x_{\mathrm{idim}}}\right],
$$

where $U^{\mathrm{n}}$ and $U^{\mathrm{n}+1}$ are the conserved variables respectively at time $t^{\mathrm{n}}$ and $t^{\mathrm{n}+1}=$ $t^{\mathrm{n}}+\Delta t^{\mathrm{n}}$ where $\Delta t^{\mathrm{n}}$ is the time step. The time-averaged fluxes $\mathbf{F}_{\text {idim }}^{\mathrm{i}+\frac{1}{2}}$ in the time interval $\left[t^{\mathrm{n}}, t^{\mathrm{n}+1}\right]$ at the interface between a cell with indices $\mathrm{i}$ and its neighbour $\mathrm{i}+1$ in the direction idim are calculated from the solution of Riemann problems. ${ }^{45}$ This is illustrated in Fig. 29.

In MHD, the Riemann problem is described by a 7-waves pattern. These seven eigenvalues correspond to the left and right going Alfvèn waves and four magnetosonic waves (two fast and two slow), and between these two propagating waves there is the entropy wave. These waves are defined in a direction idim as

\footnotetext{
45 A Riemann problem is an initial value problem of a conservative equation in fluid dynamics which involves a discontinuous distribution of the conserved variables. It leads to the derivation of characteristics or the eigenvalues of the problem corresponding to different wave solutions.
} 


$$
\begin{aligned}
& \lambda_{2,6}^{\text {idim }}=u^{\text {idim }} \mp U_{\mathrm{a}}^{\text {idim }} \\
& \lambda_{1,7}^{\text {idim }}=u^{\text {idim }} \mp U_{\mathrm{f}}^{\text {idim }} \\
& \lambda_{3,5}^{\text {idim }}=u^{\text {idim }} \mp U_{\mathrm{s}}^{\text {idim }} \\
& \lambda_{4}^{\text {idim }}=u^{\text {idim }}
\end{aligned}
$$

where the Alfvèn speed $U_{\mathrm{a}}^{\text {idim }}$, the fast $U_{\mathrm{f}}^{\text {idim }}$ and the slow $U_{\mathrm{s}}^{\text {idim }}$ speed in direction idim in ideal MHD case are defined as (Ryu et al. 1995)

$$
\begin{aligned}
U_{\mathrm{a}}^{\text {idim }} & =\frac{\left|B^{\text {idim }}\right|}{\sqrt{\rho}}, \\
\left(U_{\mathrm{f}, \mathrm{s}}^{\mathrm{idim}}\right)^{2} & =\frac{c_{\mathrm{s}}^{2}+U_{\mathrm{a}}^{2} \pm \sqrt{\left(c_{\mathrm{s}}^{2}+U_{\mathrm{a}}^{2}\right)^{2}-4 c_{\mathrm{s}}^{2} U_{\mathrm{a}}^{\text {idim }}{ }^{2}}}{2} .
\end{aligned}
$$

Here $c_{\mathrm{s}}=\sqrt{\left(\frac{\partial p}{\partial \rho}\right)}$ is the local sound speed and $U_{\mathrm{a}}=\frac{|B|}{\sqrt{\rho}}$ is the local Alfvèn speed. We can note here that the characteristic MHD waves are direction dependent. These waves are depicted in Fig. 30. The seven eigenvalues satisfy the inequalities

$$
\lambda_{1} \leq \lambda_{2} \leq \lambda_{3} \leq \lambda_{4} \leq \lambda_{5} \leq \lambda_{6} \leq \lambda_{7}
$$

However, some eigenvalues can coincide depending on the direction and strength of the magnetic field. Therefore, the MHD equations form a non-strictly hyperbolic system (Brio and Wu 1988).

\subsubsection{The Courant-Friedrichs-Lewy condition}

Courant, Friedrichs and Lewy (1928) showed that the stability of numerical schemes requires the use of all the information contained in the initial state that will influence the solution in a given spatial cell. To satisfy this condition, the ratio between the spatial discretization $\Delta x$ and the time step $\Delta t$ should be smaller than the largest velocity of the signal solution of the PDEs; i.e. $\max \left(\left|\lambda_{1}\right|,\left|\lambda_{7}\right|\right)$ (maximum speed propagating to the left and to the right). This inequality is called the CFL condition:

$$
\Delta t \leq \frac{\Delta x}{\max \left(\left|\lambda_{1}\right|,\left|\lambda_{7}\right|\right)} .
$$

Satisfying this condition is necessary for the convergence of explicit difference schemes.

\subsubsection{Riemann solvers}

The Riemann solvers are a fundamental tool in the development of FVM. They are based on a simplification of the hyperbolic equations. Moreover, at each step they simplify the physical state to constant piecewise values with jump discontinuities at some 


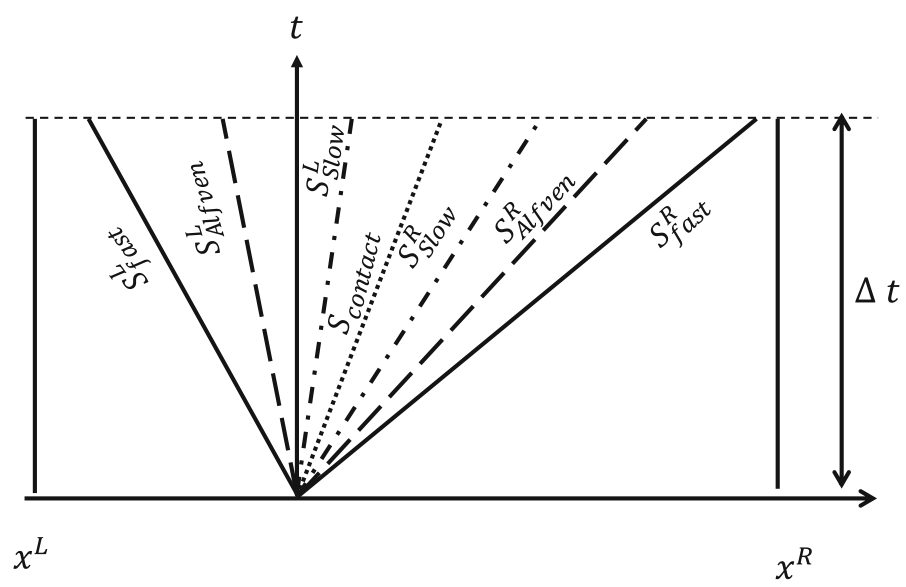

Fig. 30 The Riemann fan. See text for the nomenclature of the waves.

or all eigenvectors (and associated eigenvalues) that characterize the hyperbolic equations. In the case of MHD equations, the solution of the Riemann problem is controlled by seven waves, either discontinuities or rarefaction fans. Each wave is associated with one eigenvalue [characteristic velocities (Eq. 58)], where $\lambda_{1,3,5,7}$ are associated with shock or rarefaction waves, $\lambda_{2,6}$ are associated with rotational discontinuity and $\lambda_{4}$ is associated with a contact discontinuity (see Fig. 30).

There are different types of Riemann problem solvers (see Leveque 1998; Martí and Müller 2015):

- An exact Riemann solver requires an iterative method and thus is impractical for a MHD code (e.g., Takahashi et al. 2014; Torrilhon 2003). However, the results of the exact Riemann problem are used as reference solution to check the numerical precision and performance of approximate Riemann solvers.

- A linearized Riemann solver such as the Roe solver (Roe 1981) requires a decomposition of the left and right eigenvectors into characteristics. This solver is complex and time-consuming in MHD and HD cases. Moreover, the Roe solver can lead to negative solutions (Einfeldt et al. 1991).

- A guess-based Riemann solver such as the HLL solver (Harten-Lax-van Leer) introduces an estimate of the wave speed and the solution is averaged over the Riemann fan.

In this review, we focus on the most used Riemann solvers for MHD, all in the guessbased category. There are three main such solvers:

- The TVDLF (Total Variation Diminishing Lax-Friedrich Rusanov) Riemann solver (e.g., Bouchut 2004) is constructed by assuming a mean state which is given by the fastest wave. Indeed, the flux at cells interface $i+1 / 2$ is given by,

$$
F^{\mathrm{i}+1 / 2}=\frac{1}{2}\left[F\left(U_{\mathrm{L}, \mathrm{i}+1 / 2}\right)+F\left(U_{\mathrm{R}, \mathrm{i}+1 / 2}\right)-S_{\max }\left(U_{\mathrm{R}, \mathrm{i}+1 / 2}-U_{\mathrm{L}, \mathrm{i}+1 / 2}\right)\right],
$$


where the fastest propagating speed at cell interface is:

$$
S_{\max }=\max \left(S_{\mathrm{L}, \max }, S_{\mathrm{R}, \max }\right),
$$

with $S_{\mathrm{L}, \max }, S_{\mathrm{R}, \max }$ are the eigenvalues associated with the fastest wave propagating respectively at the left and at the right of cell interface $i+1 / 2$. They are defined as,

$$
S_{\mathrm{L} \max }=\max _{(1 \leq k \leq 7)}\left(\left|\lambda_{k, \mathrm{~L}}\right|\right), \quad S_{\mathrm{R} \max }=\max _{(1 \leq k \leq 7)}\left(\left|\lambda_{k, \mathrm{R}}\right|\right) .
$$

- The HLL solver proposed by Harten et al. (1983) is constructed by assuming an average intermediate state between the fastest and slowest waves.

$$
F^{\mathrm{i}+1 / 2}= \begin{cases}F\left(U_{\mathrm{L}, \mathrm{i}+1 / 2}\right) & S_{\mathrm{L}, \mathrm{i}+1 / 2}>0 \\ F(\mathrm{HLL}, \mathrm{i}+1 / 2) & S_{\mathrm{L}, \mathrm{i}+1 / 2} \leq 0 \leq S_{\mathrm{R}, i+1 / 2} \\ F\left(U_{\mathrm{R}, i+1 / 2}\right) & S_{\mathrm{R}, i+1 / 2}<0\end{cases}
$$

where

$$
\begin{aligned}
& F(\mathrm{HLL}, \mathrm{i}+1 / 2) \\
& =\frac{S_{\mathrm{R}, \mathrm{i}+1 / 2} U_{\mathrm{R}, \mathrm{i}+1 / 2}-S_{\mathrm{L}, \mathrm{i}+1 / 2} U_{\mathrm{L}, \mathrm{i}+1 / 2}+F\left(U_{\mathrm{L}, \mathrm{i}+1 / 2}\right)-F\left(U_{\mathrm{R}, i+1 / 2}\right)}{S_{\mathrm{R}, \mathrm{i}+1 / 2}-S_{\mathrm{L}, \mathrm{i}+1 / 2}}
\end{aligned}
$$

The outermost wave speed $S_{\mathrm{L}, \mathrm{i}+1 / 2}$ and $S_{\mathrm{R}, \mathrm{i}+1 / 2}$ are estimated using the left and right states,

$$
\begin{aligned}
& S_{\mathrm{L}}=\min _{(1 \leq k \leq 7)}\left(\lambda_{k, \mathrm{~L}}, \lambda_{k, \mathrm{R}}\right) \\
& S_{\mathrm{R}}=\max _{(1 \leq k \leq 7)}\left(\lambda_{k, \mathrm{~L}}, \lambda_{k, \mathrm{R}}\right)
\end{aligned}
$$

- The HLLC solver proposed by Toro et al. (1994) is a two-state HLL Riemann solver. It introduces sub-structures associated with a contact discontinuity into the sub-slow state of the HLL Riemann solver.

- The HLLD solver (Miyoshi and Kusano 2005) is a four-state HLL Riemann solver. The HLLD Riemann solver introduces sub-structures associated with the two rotational discontinuities $\lambda_{\mathrm{a}, \mathrm{L}}, \lambda_{\mathrm{a}, \mathrm{R}}$ separated by the contact discontinuity.

In the Riemann solvers presented above, the numerical estimation of the fluxes at interface $F^{\mathrm{i}+1 / 2}$ requires the values of the conserved variables to the left $U_{\mathrm{L}, \mathrm{i}+1 / 2}$ and to the right $U_{\mathrm{R}, \mathrm{i}+1 / 2}$ of the cell interface located at $x_{\mathrm{i}+1 / 2}$. These are reconstructed from cell centred values $U$. These spatial reconstructions can be performed by using a slope limited scheme to keep the reconstruction monotonic. There are various slope limited schemes such as minmod, super-bee and monotonized central difference limiter (MCD) (Toro 2009), ppm (Colella and Woodward 1984), KOREN (Koren and van der 
Maarel 1993), van Leer (van Leer 1979). The minmod limiter is the most stable in the presence of strong discontinuities and is very efficient in decreasing numerical instabilities. MCD and Super-bee limiters are more efficient in the vicinity of smooth flows because they permit to retrieve a centered slope. PPM and KOREN are higher order limiters and thus with lower numerical dissipation, they can be used on large classes of problems with smooth flow, intermediate discontinuities and some strong shocks.

Numerical resolution of the partial differential Eq. (52) also requires high order temporal accuracy. This is realized by using a second order predictor-corrector scheme or a higher order scheme such as the strong stability preserving Runge-Kutta scheme.

\subsubsection{Semi-implicit and implicit schemes}

The various complex physical processes associated with astrophysical plasma phenomena act on widely different time scales. The precise treatment of these phenomena with an explicit MHD approach relies on the accuracy to which one can capture the dynamics as imposed by the CFL condition. In some cases, the resulting short timeresolution can make the MHD simulation computationally intractable with the explicit method. To make these simulations computationally feasible, it is necessary to integrate the MHD equations with larger time steps. This is possible when we are not interested in tracking all fast waves in the system and we can step over some unimportant propagating waves. This can be realized by the use of implicit schemes, which are preferable for complex astrophysical MHD simulations. However, parallel computation with implicit schemes becomes less efficient than with explicit schemes since implicit schemes use iterative algorithms that request more communication between the different processes.

In the implicit method the original system of differential Eq. (54) is rewriten as follows:

$$
\partial_{t} \mathbf{U}=R(\mathbf{U}),
$$

where $R(U)=-\partial_{\mathrm{i}} \mathbf{F}^{\mathrm{i}}(\mathbf{U})+S(U)$ and $S(U)$ represents source terms. $R(U)$ is a non-linear function of $U$. Eq. (67) is usually discretized in time using a third-level Backward Differentiation Formula method

$$
U^{n+1}=U^{n}+\Delta t\left[\beta \mathcal{R}\left(U^{n+1}\right)+(1-\beta) \frac{U^{n}-U^{n+1}}{\Delta t^{n-1}}\right],
$$

where $\beta=\left(\Delta t^{n}+\Delta t^{n-1}\right) /\left(2 \Delta t^{n}+\Delta t^{n-1}\right)$. In the case of constant time steps $\beta=$ $2 / 3$ and Eq. (68) is simplified to a second-order Backward Differentiation formula; with $\beta=1$ Eq. (68) corresponds to a backward-Euler scheme. In the case $\beta=0.5$ the scheme is fully implicit.

In the implicit method, at each time step, the multidimensional nonlinear system

$$
G\left(U^{n+1}\right)=U^{n+1}-\left\{U^{n} \Delta t\left[\beta \mathcal{R}\left(U^{n+1}\right)+(1-\beta) \frac{U^{n}-U^{n+1}}{\Delta t^{n-1}}\right]\right\}=0,
$$

must be solved to determine the time-updated solution for the state $U^{n+1}$. 
In some specific simple cases, such as 2D isothermal hydrodynamics, Eq. (69) can be preconditioned analytically to obtain a diagonal matrix, leading to a system of linear equations that can be solved by relaxation techniques (Harlow and Amsden 1968). The linearization (analytical preconditioning) of Eq. (69) can be done either about the initial equilibrium $G\left(U^{n}\right)$ or about the current state in order to construct the implicit operator $G\left(U^{n+1}\right)=0$ needed to advance to the next time step. These classes of implicit schemes are used for specific problems and geometry (e.g., Harned and Schnack 1986). Another class of implicit schemes uses Newton-Krylov techniques for the resolution of nonlinear systems like Eq. (69). Newton's method consists of the local linearization of Eq. (69) for each state $U^{\mathrm{n}(\mathrm{m})}$ at iteration $m$ according to

$$
G\left(U^{\mathrm{n}(\mathrm{m})}\right)=\left(U^{\mathrm{n}(\mathrm{m}-1)}\right)+\frac{\partial G(U)}{\partial U}\left(U^{\mathrm{n}(\mathrm{m})}-U^{\mathrm{n}(\mathrm{m}-1)}\right)+\mathcal{O}\left(\Delta t^{2}\right)
$$

which can be substituted into Eq. (69) giving the equation to be solved,

$$
\left(I-\Delta t \beta \frac{\partial G(U)}{\partial U}\right) \delta U=\Delta t\left(\beta U^{\mathrm{n}(\mathrm{m}-1)}+(1-\beta) \frac{U^{\mathrm{n}(\mathrm{m}-1)}-U^{\mathrm{n}(\mathrm{m}-2)}}{\Delta t^{\mathrm{n}(\mathrm{m}-2)}}\right),
$$

and after the values of $\mathrm{U}$ is updated as

$$
U^{n+1}=U^{n}+\delta U
$$

However, this nonlinear resolution method benefits tremendously from accurate initial guesses, $U^{n(0)}$. In many cases an explicit predictor is used to provide an initial guess to the implicit scheme (e.g., Reynolds et al. 2006). The iterative resolution of the linear Eq. (71) is performed in general by using preconditioned Krylov (sub)space solvers (e.g., Tóth et al. 2006) with the non-restarted generalized minimum residual method (GMRES) iterative solver (Brown and Hindmarsh 1989). These linear solvers are very efficient for large-scale problems since they do not require storage of the matrix.

\subsubsection{Magnetic divergence-free algorithms}

The resolution of Euler and Maxwell equations using the standard Godunov schemes does not work by default in maintaining the divergence-free property of the magnetic field $\nabla \cdot \mathbf{B}=0$. The resulting error accumulated during the simulation may grow to the point that it produces unphysical forces (Tóth 2000). Several strategies have been undertaken to handle the magnetic field evolution in numerical MHD. They are classified into two main categories.

We first find the divergence-cleaning schemes, where the evolution of magnetic field components is treated as any other MHD variables and only in a second step a divergence-cleaning procedure is applied. In these schemes the magnetic field components are defined at cells center as others variables. Then, the MHD equations are solved by adding source terms function of $\nabla \cdot \mathbf{B}$. There are various methods for divergence-cleaning schemes, such as the Generalized Lagrange Multiplier (GLM) 
(Dedner et al. 2002). In the GLM method, a new transport variables $\Psi$ and its governing equation is introduced into the MHD equations system, which plays the role of advection and dissipation of the local divergence error. The divergence-cleaning can be treated as well by the eight-wave formulation approach Powell et al. (1999). There is also the projection method (Brackbill and Barnes 1980). In this scheme at each iteration the Poisson equation $\nabla^{2} \Phi=-\nabla \cdot \mathbf{B}$ is solved and in the second step the magnetic divergence part is removed form the magnetic field $\mathbf{B}=\mathbf{B}-\nabla \Phi$. Let us also mention the vector divergence-cleaning scheme (Balsara 1998) which is an extension of the projection method. Finally, another divergence-cleaning scheme based on the use of an artificial diffusivity added at each time step, following the completion of the TVD Lax-Friedrich scheme (van der Holst and Keppens 2007), where a term $\eta \nabla \cdot \nabla \cdot \mathbf{B}$ is added to the magnetic field to diffuse the magnetic divergence.

A second category of schemes is based on constrained transport methods, originally introduced by Evans and Hawley (1988). Such schemes use a staggered mesh formulation which is inherently divergence-free. In this method the magnetic field is defined at face centers and the remaining fluid variables are defined at cell centers. In this approach, the electric field is set along the cell edges. This method sustains a specified discretization of the magnetic field divergence around machine round off error.

The constrained transport method is attractive from a physical point of view, however, it requires specific treatment for magnetic field variables different from others variables, which is inconvenient for implementation specifically in the AMR. The diffusive method reduces the numerical error of $\nabla \cdot \mathbf{B}$ by adding a source term in the induction equation and the energy equation. The projection method involves an additional Poisson equation which significantly increases the computational cost. The GLM method is based on the use of central cell magnetic field and thus it can easily be applied on general grids.

\subsubsection{Adaptive mesh refinement techniques}

The numerical resolution of the PDEs (Eq. 57) uses a discrete domain. Therefore its precision depends on the mesh resolution (spacing), determined according to the scales of the phenomenon under study. In fluid mechanics a broad variety of spatial perturbations exist and can interact with each other. The complexity of these interactions requires the resolution of the problem at all scales. With uniform meshes, if high resolution is required throughout the computational domain the simulation can become computationally extremely costly.

Adaptive Mesh Refinement (AMR) addresses the problem of resolving this wide range of scales by increasing the spatial resolution around small scale structures. It is achieved by increasing locally the mesh resolution and then adjusting the computational effort locally to maintain a uniform level of accuracy throughout the computational domain. This type of AMR approach is called the $h$-type refinement. It consists in the splitting of existing elements into smaller ones. The development and use of the AMR starts with Berger and Oliger (1984), the transition from serial to parallel computing occurred after Griebel and Zumbusch (1999). Various AMR approaches exist, depending on the cells shape and the logical grouping of the cells 
on the mesh: gathering the cells according to their size $h$, inducing a particularly strict ansatz in the hierarchy, $h=n^{-\mathrm{L}}$, corresponding to some refinement level $L$ ( $\mathrm{L}$ is an integer). The cells volume at level $l$ are $h^{-\mathrm{ND}}$ (ND = number of dimensions) smaller than the cells at coarse level $l=1$. The most used hierarchy is $n=2$ where the coarse cell is divided by $2^{\mathrm{ND}}$ when it is refined.

In the block structured methods the cells are arranged in blocks according to their levels only. This method does not have any constraints on the size or shape of these blocks. Tree-base methods impose constraints on the block size and shape. With this method, the blocks are organized hierarchically as a quadtree in 2D (octree in 3D). In this distribution, the blocks in use at level $l$ represent the tree leaves since they have no children and they have an associated parent (ascendant) blocks. The parent blocks that are at the lowest refinement level $l=1$ represent the tree roots. In some simulations, there is a need to use multiple tree roots arranged in an unstructured AMR, giving rise to a forest of trees.

\subsubsection{Errors estimator}

The AMR consists in the use of a coarse grid over the entire computational domain and refined grids only in some specific regions where the local truncation errors are judged to be too large to maintain a given numerical accuracy. These errors can be computed using the Richardson-estimator which compares the evolution of the variables at two successive levels. This estimator is accurate however it requires a lot of memory and is time consuming. Another estimator is the Löhner-estimator (Lohner 1987), a modified central second derivative normalized by the sum of first-order forward and backward gradients. It has the advantage of using mostly local calculations of any variables of the simulation and their combinations.

\subsubsection{Load balance}

In the simulation box, the distribution of blocks across the processors requires the use of a space filling curve. The most used space filling curves (SFCs) are Hilbert, Peano, and Sierpinski curves (Bader 2013). The Hilbert and Peano curves use recursive algorithms. The Morton order is also widely used due to its ease of implementation in the space filling. However the curves it generates are not continuous and thus do not fit into the family of finite SFCs.

With this curve the blocks are organized over a forest of trees and thus they are distributed over all the processors. There are two approaches for this forest partition. In the first approach, each tree and its leaves are associated to one owner process. In the second approach, the tree leaves can belong to multiple processes. The first approach is simple to implement however it does not provide a right load balance since the number of blocks per process may differ. The second approach, even if it presents issues with shared tree between processes, provides a perfect load balance, the difference in block distribution over processes is at most one. This last approach provides the best scalability (Keppens et al. 2012). 

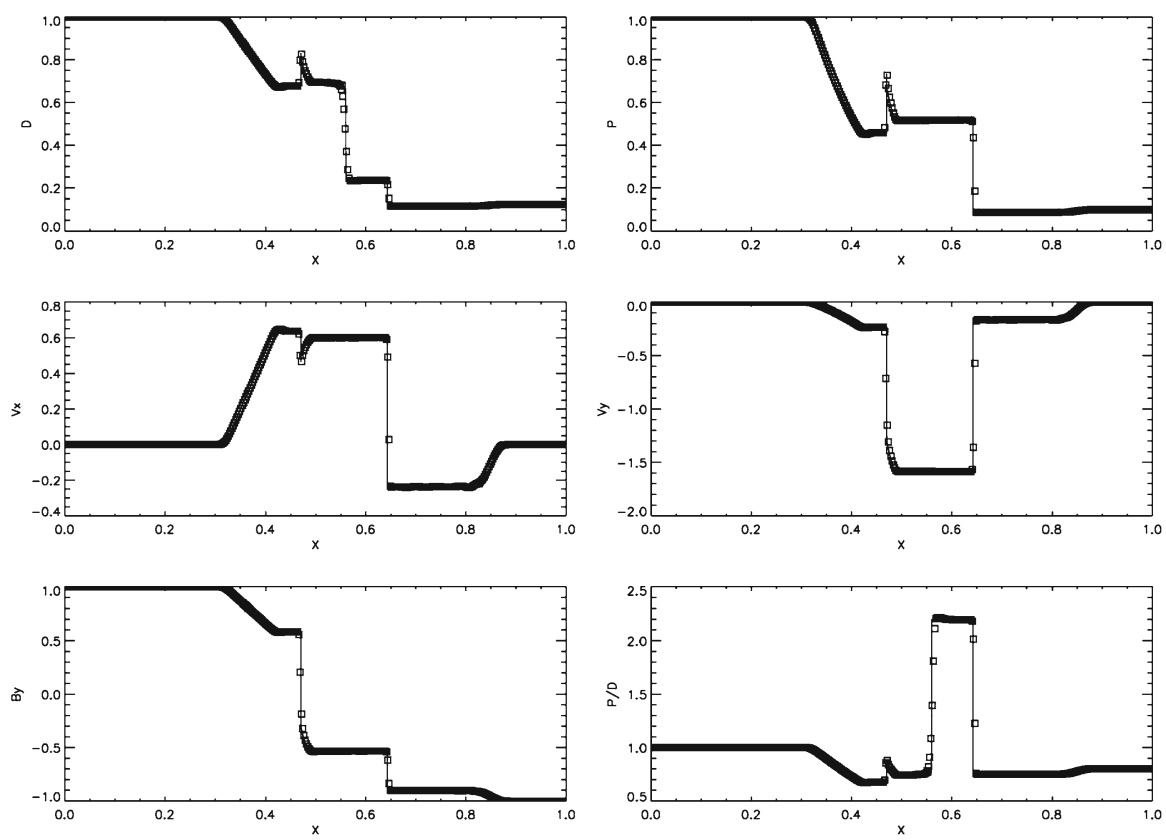

Fig. 31 Sod shock tube test in MHD. The density, pressure, velocity components, transverse component of the magnetic field, and specific internal energy [scaled by $\left(\gamma_{\mathrm{ad}}-1\right)$ ] for the Brio \& Wu (1988) shock tube problem are plotted at $t=0.08$, computed with 400 grid points, second-order spatial reconstruction, and Roe fluxes. The solid line is a reference solution computed with 104 grid points. Image reproduced with permission from Stone et al. (2008), copyright by AAS

\subsubsection{Standard numerical tests}

The development of numerical tools requests extended tests for all implemented physics and algorithms. Many comprehensive and well documented sets of tests have been presented in the literature. Tests are set for all physics, all dimensions, and implemented geometries and AMR schemes. For many 1D tests an exact solution exists and it is possible to compute the deviation of the numerical result from it using some error norm (e.g., norm error $L_{1}=\Sigma_{i}\left\|q_{i}-q_{i}^{0}\right\| / N$, where $N$ is number of point, $q_{i}^{0}$ is the exact solution at cell $i, q_{i}^{0}$ is the numerical result at cell $i$ ).

One of the standard one-dimensional tests is the Sod MHD shock tube (Brio and Wu 1988). The shock tube test consists of two constant states, one on the left and the second on the right, separated by a discontinuity. This test allows to check the ability of a numerical code to treat correctly the Riemann problem in $1 \mathrm{D}$ and resolve the Riemann fan evolution, see Fig. 31.

For two-dimensional MHD tests, one of the standard tests in the vortex of Orszag \& Tang (1979), see Fig. 32. This test consists in a doubly periodic fluid configuration leading to 2D supersonic MHD turbulence. The density and pressure are set to constant values, while the velocity and magnetic field are set as 


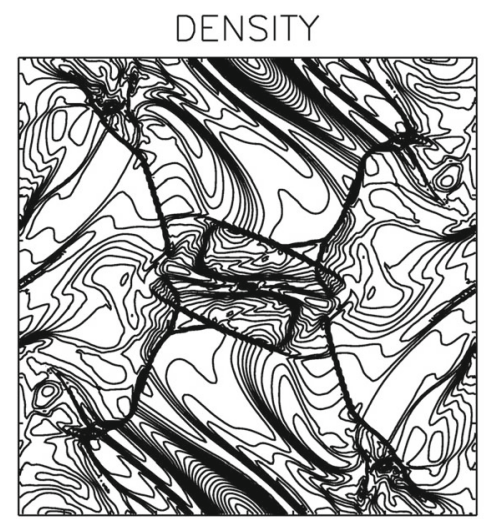

MAGNETIC PRESSURE

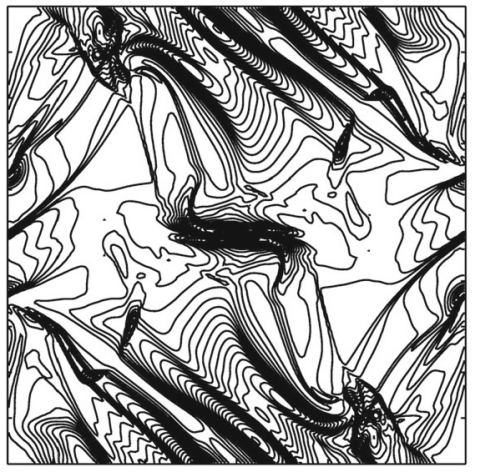

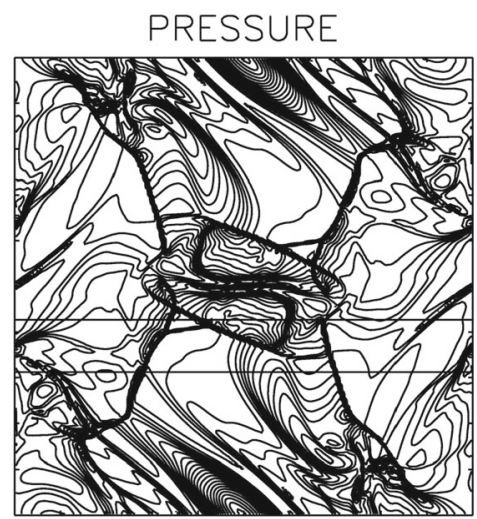

SPECIFIC KINETIC ENERGY

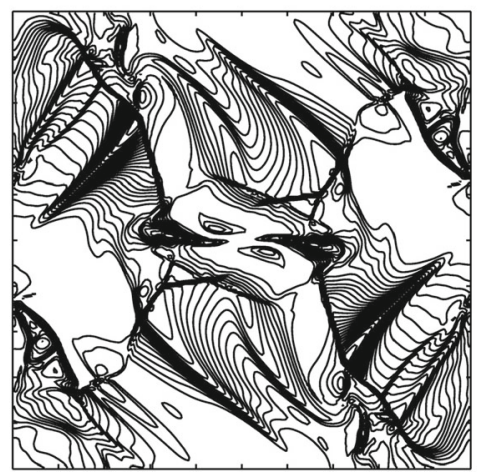

Fig. 32 Orszag-Tang vortex test. Contours of selected variables at $t_{f}=1 / 2$, computed using a grid of $192 \times 192$ cells, third-order reconstruction, and Roe fluxes. Thirty equally spaced contours between the minimum and maximum are used for each plot. Image reproduced with permission from Stone et al. (2008), copyright by AAS

$$
\begin{aligned}
\mathbf{u} & =(-\sin y, \sin (x), 0), \\
\mathbf{B} & =(-\sin y, \sin (2 x), 0) .
\end{aligned}
$$

This test does not have an analytical solution and the results have to be compared between different codes.

\subsubsection{Relativistic magnetohydrodynamics}

The MHD description introduced in the previous paragraphs is relevant for nonrelativistic plasma velocities and energies. It is adequate for plasmas in the interstellar medium and in the vicinity of stars. However, some astrophysical phenomena involve relativistic plasma flows with energies of the order of the mass energy. In order to model these plasmas in the framework of the fluid model, the MHD formulation has to be revised using the special relativity (SR) framework. In flat space-time, the plasma is described by the following SR-MHD equations (see Martí and Müller 2015) 


$$
\begin{gathered}
\partial_{\mathrm{t}}(\gamma \rho)+\nabla \cdot(\rho \Gamma \mathbf{u})=0 \\
\partial_{\mathrm{t}}\left(\gamma^{2} h \mathbf{u}+\mathbf{E} \times \mathbf{B}\right)+\nabla \cdot\left(\gamma^{2} h \mathbf{u u}-\mathbf{E} \mathbf{E}-\mathbf{B B}+p \mathbf{I}\right)=\mathbf{0}, \\
\partial_{t}\left(h \gamma^{2}-p-\gamma \rho+\frac{E^{2}+B^{2}}{2}\right)+\nabla \cdot\left(\left(h \gamma^{2}-p-\gamma \rho\right) \mathbf{u}+\mathbf{E} \times \mathbf{B}\right)=0 \\
\partial_{\mathrm{t}} \mathbf{B}+\nabla \cdot(\mathbf{u B}-\mathbf{B u})=\mathbf{0}
\end{gathered}
$$

where the closure of this system of equations is provided by the equation of state expressed with the enthalpy $h=h(\rho, p)$. The total pressure is $p_{\mathrm{t}}=p+\frac{E^{2}+B^{2}}{2}$ where the electric field is given by Ohm's law. In the ideal case $\mathbf{E}=-\mathbf{u} / c \times \mathbf{B}$, finally $\Gamma=\left(1-(u / c)^{2}\right)^{-1 / 2}$ is the Lorentz factor of the flow.

The numerical resolution of SR-MHD equations exploits the same type of algorithms as presented before, by solving Eq. (74) in conservative form

$$
\partial_{\mathrm{t}} U+\partial_{\mathrm{i}} F^{\mathrm{i}}(U)=0,
$$

where the conserved variables are

$$
U=\left(\begin{array}{c}
\gamma \rho \\
h \gamma^{2} \mathbf{u}+\mathbf{E} \times \mathbf{B} \\
h \gamma^{2}-p-\gamma \rho+\frac{E^{2}+B^{2}}{2} \\
\mathbf{B}
\end{array}\right),
$$

and the corresponding flux tensor

$$
F(U)=\left(\begin{array}{c}
\gamma \rho \mathbf{u} \\
h \gamma^{2} \mathbf{u u}-\mathbf{E} \mathbf{E}-\mathbf{B B}+p \mathbf{I} \\
\left(h \gamma^{2}-p-\gamma \rho\right) \mathbf{u}+\mathbf{E} \times \mathbf{B} \\
\mathbf{u B}-\mathbf{B u}
\end{array}\right) .
$$

Most of the complications in relativistic MHD comes from the non-linear relation between the primitive variables $(\rho, \mathbf{u}, p)$ and conserved variables $U$ :

$$
\begin{gathered}
\xi-p-\tau-D+B^{2}-\frac{1}{2}\left(\left(\frac{B}{\gamma}\right)^{2}+\left(\frac{\mathbf{S} \cdot \mathbf{B}}{\xi}\right)^{2}\right)=0 \\
\mathbf{u} \cdot \mathbf{B}=\frac{\mathbf{S} \cdot \mathbf{B}}{\xi} \\
\frac{1}{\gamma^{2}}=1-\frac{(\mathbf{S}+(\mathbf{u} \cdot \mathbf{B}) \mathbf{B})^{2}}{\left(\xi+B^{2}\right)^{2}} \\
\mathbf{u}=\frac{\mathbf{S}+(\mathbf{u} \cdot \mathbf{B}) \mathbf{B}}{\xi+B^{2}}
\end{gathered}
$$

where $D=\gamma \rho$ is laboratory frame density, $\xi=\gamma^{2} \rho h, \mathbf{S}=h \gamma^{2} \mathbf{u}+\mathbf{E} \times \mathbf{B}$ the momentum and $\tau=h \gamma^{2}-p-\gamma \rho+\frac{E^{2}+B^{2}}{2}$. Eq. (78) can be handled only numerically, 
the most used scheme is the Newton-Raphson method (Noble et al. 2006). The iteration is performed on the pressure, on the enthalpy or the velocity. In SR hydrodynamics the iterative method can be avoided and instead a quartic equation can be solved (Schneider et al. 1993).

As in classical MHD, the SRMHD schemes exploits characteristic speed of plasma normal modes, but these are limited by the light speed, i.e.

$$
-1 \leq \lambda_{1} \leq \lambda_{2} \leq \lambda_{3} \leq \lambda_{4} \leq \lambda_{5} \leq \lambda_{6} \leq \lambda_{7} \leq 1
$$

For a specific direction idim, the entropy wave as in classical MHD travels with speed $\lambda_{4}=v^{\text {idim }}$, the Alfvén wave has speed

$$
\lambda_{2,6}=u^{\mathrm{idim}} \mp \frac{B^{\text {idim }}}{\sqrt{\left(\rho h+B^{2}\right) \mp(v \cdot B)}},
$$

and the magneto-acoustic speeds are found from the quartic equation

$$
\begin{aligned}
& \rho h\left(1-c_{\mathrm{S}}\right) \gamma^{4}\left(\lambda-u^{\mathrm{idim}}\right)^{4}-\left(1-\lambda^{2}\right) \times \\
& \left(\gamma^{2}\left(\rho h c_{s}^{2}-B^{2}\right)\left(\lambda-u^{\mathrm{idim}}\right)^{2}-c_{s}^{2}\left(\Gamma(u \cdot B)\left(\lambda-u^{\mathrm{idim}}\right)-\frac{B^{\mathrm{idim}}}{\gamma}\right)^{2}\right)=0
\end{aligned}
$$

where $c_{\mathrm{S}}$ is the sound speed. In the hydrodynamics case, Eq. (81) becomes a single quadratic expression, and then the use of exact analytic formulae for the root evaluation is straightforward. In SRMHD Eq. (81) is quartic, although an analytical solution exists (Del Zanna et al. 2003), it is more easily obtained by numerical iteration, for which a Laguerre method can be used.

Standard numerical tests In special relativistic hydrodynamics and magnetohydrodynamics several numerical experiments are used as benchmark tests. Like in classical HD and MHD there is the Sod shock tube and the equivalent of the Orzag-Tang vortex (see above). Here we show the rotor test (Del Zanna et al. 2003), in Fig. 33. It consists in a disk of radius 0.1 with higher density, $\rho=10$, positioned at the center of the computational domain $[0,1] \times[0,1]$, rotating at high relativistic speed, $\Omega=9.95$, thus the Lorentz factor at the disk edge $\Gamma_{\max } \simeq 10.0$, the rotor is embedded in a static background with $\rho=1, p=1$, and uniform magnetic field $B_{x}=1$ with polytropic index $\gamma=5 / 3$.

\subsection{Multi-fluid methods}

The previous section presented the case of a single fluid. However, in astrophysics the thermal plasma pressure is usually in competition with the pressure in other components: cosmic rays, radiation fields, dust, neutral species, or requires to include an 

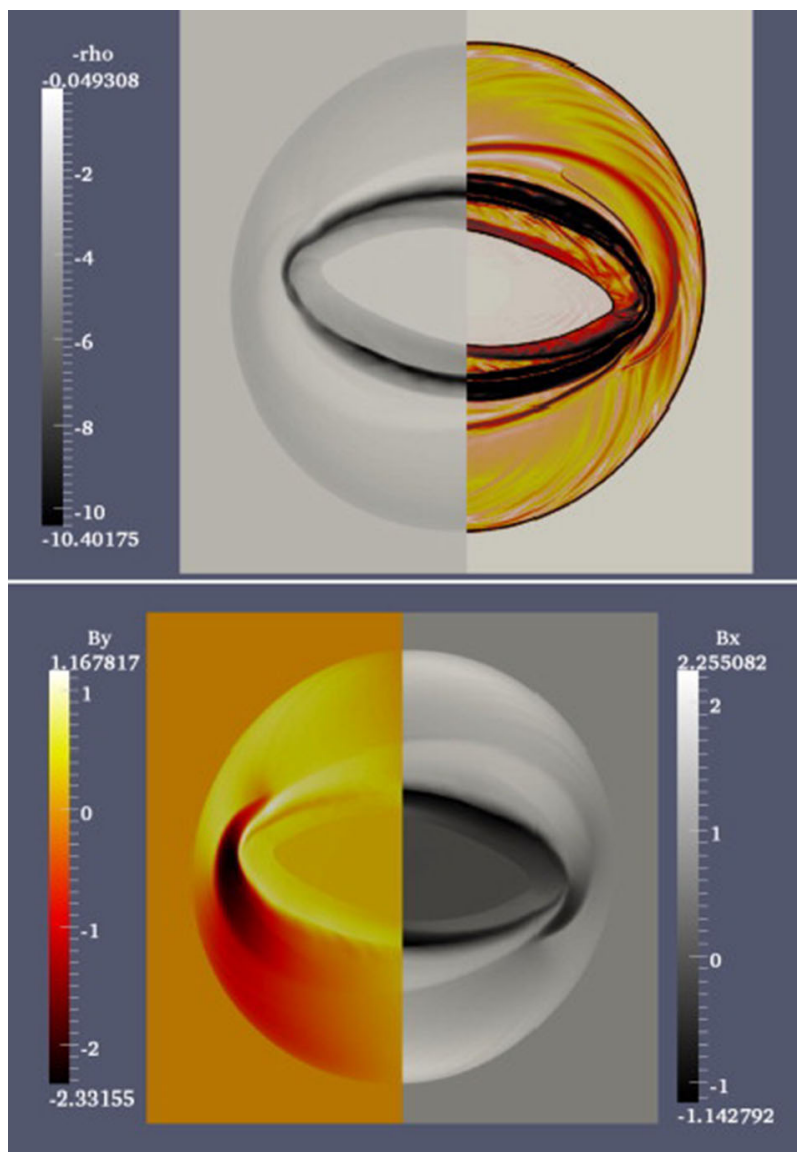

Fig. 33 The relativistic rotor problem. The top panel shows the density structure (both in linear scale as well as using a Schlieren plot), the bottom panel the magnetic field components, at time $t=0.4$. Image reproduced with permission from Keppens et al. (2012), copyright by Elsevier

electron fluid. In bi- or multi-fluid models it is in principle necessary to add as many fluid equations as the number of species to be included in the simulations, and to account for the friction forces induced by collisions among the species in the momentum conservation equation (see, e.g., O'Sullivan and Downes 2007).

As in this review we are mostly interested in non-thermal particle acceleration and transport we restrict our discussion to the case of the interaction of a thermal fluid and a non-thermal (or cosmic ray) component (Drury and Voelk 1981; Rasera and Chandran 2008).

\subsubsection{Model equations}

CRs impact fluid dynamics through the effect of the gradient of their pressure $p_{\mathrm{CR}}$ (Eq. 9). The momentum equation is modified as 


$$
\partial_{t}(\rho \mathbf{u})+\nabla \cdot\left(\rho \mathbf{u u}-\mathbf{B B}+\left(p+p_{\mathrm{CR}}+\frac{B^{2}}{2}\right) \mathbf{I}\right)=0,
$$

and the energy equation now includes the work of this force plus another component produced by the $\mathrm{CR}$ diffusion

$$
\partial_{t}(e)+\nabla \cdot\left(\left(e+p+p_{\mathrm{cr}}+\frac{B^{2}}{2}\right) \mathbf{u}-(\mathbf{B} \cdot \mathbf{u}) \mathbf{B}+\mathbf{F}_{\mathrm{CR}}\right)=0
$$

where the CR flux is $\mathbf{F}_{\mathrm{CR}}=-\overline{\bar{D}} \cdot \nabla e_{\mathrm{CR}}$ and $e_{\mathrm{CR}}$ is the $\mathrm{CR}$ energy density. The $\mathrm{CR}$ diffusion coefficient can be decomposed into parallel and perpendicular components with respect to the background magnetic field, namely

$$
D_{i j}=D_{\perp} \delta_{i j}+\left(D_{\|}-D_{\perp}\right) b_{i} b_{j}
$$

where $\mathbf{b}=\mathbf{B} / B$ (Hanasz and Lesch 2003). An energy equation for the CR energy density $e_{\mathrm{CR}}$ is now required. It reads

$$
\partial_{t}\left(e_{\mathrm{CR}}\right)+\nabla \cdot\left(e_{\mathrm{CR}} \mathbf{u}+\mathbf{F}_{\mathrm{CR}}\right)=-p_{\mathrm{CR}} \nabla \cdot \mathbf{u} .
$$

CR pressure and energy density are linked by $p_{\mathrm{CR}}=\left(\gamma_{\mathrm{CR}}-1\right) e_{\mathrm{CR}}$, where a CR gas adiabatic index $\gamma_{\mathrm{CR}}$ is introduced. The above energy equation does not have a fluxconservative form. Kudoh and Hanawa (2016) (see also Pfrommer et al. 2006) propose an alternative approach leading to a full set of flux-conservative equations for the CRMHD system. To proceed the authors introduce the CR mass density $\rho_{\mathrm{CR}}=p_{\mathrm{CR}}^{1 / \gamma_{\mathrm{CR}}}$, thus approximating CRs as a polytropic gas. With this assumption the CR energy equation can be recast into a continuity equation for the $\mathrm{CR}$ gas

$$
\partial_{t}\left(\rho_{\mathrm{CR}}\right)+\nabla \cdot\left(\rho_{\mathrm{CR}} \mathbf{u}\right)=0
$$

The previous equation implies that that $\rho_{\mathrm{CR}} / \rho$ is conserved along a streamline.

\subsubsection{Specific numerical schemes of CR-fluid systems}

The new set of equations can be solved using FVM as for standard MHD equations. The solver now has to account for the $\mathrm{CR}$ pressure which modifies the local sound speed $c_{\mathrm{s}}=\sqrt{\gamma_{\mathrm{ad}} P / \rho+\gamma_{\mathrm{CR}} P_{\mathrm{CR}} / \rho}$. The $\mathrm{CR}$ pressure is dominated by the relativistic part of the $\mathrm{CR}$ distribution hence $\gamma_{\mathrm{CR}} \simeq 4 / 3$. The standard solvers detailed in Sect. 5.2.2 can be used to treat the above CR-MHD system. Working in the framework of the fluid-kinetic approach (Sect. 5.4), Miniati (2007) develop a modified GlimmGodunov solver where the CR mediation is included in the Riemann problem. Kudoh and Hanawa (2016) propose a CR+MHD solver, second order accurate in space and time for a bi-fluid system based on a Roe solver. 
Semi-implicit and implicit methods An important difficulty of explicit schemes for CR-HD or CR-MHD systems comes from the CFL stability criterion for the CR diffusion, which imposes a timestep $\Delta t \leq \mathrm{X}_{\mathrm{CFL}} \Delta x^{2} /(2 D)$, where $\mathrm{X}_{\mathrm{CFL}}$ is the CFL number. This criterion, because the time step scales non-linearly with the grid resolution, imposes severe slowing down limitations, especially in multi-scale problems where AMR is active. Several semi-implicit or implicit approaches have been proposed to cure this issue, usually in the context of thermal conduction studies (Balsara et al. 2008). The CFL constraint from CR diffusion is alleviated by calculating the diffusion operator using an implicit method (see Sect. 5.2.3 and next). Another way to reduce the computation time is the so-called super-time stepping technique (O'Sullivan and Downes 2007; Balsara et al. 2008) where the CFL condition is imposed over a large time interval composed of multiple elementary substeps over which the stability condition can be relaxed. To increment the guessed solutions at every substep from the previous guess a Runge-Kutta method is applied, using a polynomial recursion relation (using either Chebyshev or Legendre polynomials).

Here we discuss more specifically the implicit scheme proposed by Dubois and Commerçon (2016) which is well-adapted to simulations with AMR. The diffusion operator in the energy Eq (83) can be discretized as (we write it here in 2D for a Cartesian grid but it can easily be generalized to 3D)

$$
e_{\mathrm{i}, \mathrm{j}}^{\mathrm{n}+1}=e_{\mathrm{i}, \mathrm{j}}^{\mathrm{n}}-\frac{\Delta t}{\Delta x}\left(F_{\mathrm{i}+1 / 2, \mathrm{j}}^{\mathrm{n}+1}+F_{\mathrm{i}, \mathrm{j}+1 / 2}^{\mathrm{n}+1}-F_{\mathrm{i}-1 / 2, \mathrm{j}}^{\mathrm{n}+1}-F_{\mathrm{i}, \mathrm{j}-1 / 2}^{\mathrm{n}+1}\right)
$$

where the energy density is calculated at the cell center $(i, j)$ and the fluxes are obtained at cell interfaces. The quantities are expressed at the final time step $t^{\mathrm{n}+1}$, forming a linear system that can be solved by matrix inversion. In this scheme the anisotropic part of the fluxes at the cell interfaces are calculated from the fluxes at the cell corners, i.e., $F_{\mathrm{i}+1 / 2, \mathrm{j}}^{\mathrm{n}+1}=0.5\left(F_{\mathrm{i}+1 / 2, \mathrm{j}+1 / 2}^{\mathrm{n}+1}+F_{\mathrm{i}+1 / 2, \mathrm{j}-1 / 2}^{\mathrm{n}+1}\right)$. The anisotropic part of the flux corresponds to the diffusion along the background magnetic field $D_{\|} \mathbf{b b}$. $\nabla e$.

Cosmic-ray streaming regularization CRs are not exactly advected with the background fluid but with the scattering centers (MHD waves) carried by the background fluid (Skilling 1975a). Considering slab-type waves that CRs can self-generate as they stream along the background magnetic field, we find a streaming velocity

$$
\mathbf{u}_{\mathrm{st}}=\mathbf{u}+\left\langle\frac{3}{2}\left(1-\mu^{2}\right) \frac{v_{+}-v_{-}}{v_{+}+v_{-}}\right\rangle \mathbf{u}_{\mathrm{A}},
$$

where $\mathbf{u}$ and $\mathbf{u}_{\mathrm{A}}$ are resp. the fluid and waves speed, $\mu$ is the CR pitch-angle cosine, and $\nu_{ \pm}$are the angular scattering frequency produced by the forward/backward $(+/-)$ propagating waves along the background magnetic field. This calculation assumes that the quasi-linear theory of CR transport applies (Schlickeiser 2002). A detailed calculation of this velocity requires to know the scattering frequencies, which is only possible by adding two other energy equations for each type of propagating wave. This is the purpose of the next paragraph. However, self-generated waves are preferentially produced when the local CR pressure is in excess with respect to the gas and magnetic 
pressure (as is likely the case close to CR sources), along a CR gradient. In that case, waves are preferentially triggered in one direction and one can write $\mathbf{u}_{\mathrm{st}}=$ $\mathbf{u}-\mathbf{u}_{\mathrm{a}}$ B. $\nabla p_{\mathrm{CR}} / \mid$ B. $\nabla p_{\mathrm{CR}} \mid$ (Pfrommer et al. 2017). The CR energy Eq. (85) needs to be modified according to

$$
\partial_{t}\left(e_{\mathrm{CR}}\right)+\nabla \cdot\left(e_{\mathrm{CR}} \mathbf{u}_{\mathrm{st}}+\mathbf{F}_{\mathrm{CR}}\right)=-p_{\mathrm{CR}} \nabla \cdot \mathbf{u}_{\mathrm{st}} .
$$

This equation is non-linear as $\mathbf{u}_{\mathrm{st}}$ depends on $\nabla e_{\mathrm{cr}}$. The LHS of Eq. (89) resembles an advection equation but with a speed dependent on the sign of the gradient of the CR energy density, which introduces some spurious oscillations at extrema where the gradient changes its sign (Sharma et al. 2010). These authors propose a regularization of the energy equation by replacing the sign of B. $\nabla p_{\mathrm{CR}}$ in Eq. (89) with a smooth function. The drift speed is rewritten as

$$
u_{\text {drift }}=u_{\mathrm{A}} \tanh \left(\frac{X}{\epsilon}\right) \text {, }
$$

where $X=\mathbf{B} . \nabla p_{\mathrm{CR}} /\left|\mathbf{B} . \nabla p_{\mathrm{CR}}\right|$, and $\epsilon$ is a small parameter to be adjusted. The energy equation becomes diffusive at streaming speed extrema with a diffusion coefficient dependent on $\epsilon$. Sharma et al. (2010) show that an implicit non-linear integration scheme (see their Eq. 3.9) produces a rapid convergence, but requires a sufficiently small time step to be adjusted with the value of $\epsilon$. Jiang and Oh (2018) propose an alternative approach where Eq. (89) is replaced by a system of two equations

$$
\begin{aligned}
\partial_{t}\left(e_{\mathrm{CR}}\right)+\nabla \cdot\left(\boldsymbol{\Phi}_{\mathrm{CR}}\right) & =-p_{\mathrm{CR}} \nabla \cdot \mathbf{u}_{\mathrm{st}} \\
\frac{1}{u_{\mathrm{m}}^{2}} \partial_{t}\left(\boldsymbol{\Phi}_{\mathrm{CR}}\right)+\nabla P_{\mathrm{CR}} & =-\frac{1}{D} \boldsymbol{\Phi}_{\mathrm{CR}},
\end{aligned}
$$

where $\boldsymbol{\Phi}_{\mathrm{CR}}=\mathbf{F}_{\mathrm{CR}}+e_{\mathrm{CR}} \mathbf{u}_{\mathrm{st}}$ is the total CR flux and $U_{\mathrm{m}}$ is a speed in practice taken larger than the maximum natural mode speed of the plasma. In this approach the problematic term $\operatorname{sgn}(X)$ is replaced by $\nabla . \Phi_{\mathrm{CR}}$. The system is closed by choosing the diffusion coefficient as $D=D_{0}-\mathbf{u}_{\mathrm{st}}\left(e_{\mathrm{CR}}+p_{\mathrm{CR}}\right) /\left(\mathbf{b} . \nabla p_{\mathrm{CR}}\right)$, where $D_{0}$ is a background coefficient produced by large-scale injected turbulence. Thomas and Pfrommer (2019) criticize the two previous methods. They present CR transport mediated by self-generated waves including an accurate description of CR pitch-angle scattering up to the second order in accuracy in $\left(u_{\mathrm{a}} / c\right)$. The latter is possible because of the addition of two supplementary energy equations respectively for forward and backward propagating waves (see the next paragraph). The authors employed a system of equations similar to Eq. (91) but with supplementary terms derived from the effect of CR scattering off self-generated waves.

A four-fluid approach A more complete description of the CR-fluid system includes the description of the forward and backward propagating CR self-generated waves (Skilling 1975a). It includes two supplementary fluid energy equations (Ko 1992). Now the total pressure includes the contribution of forward (backward) waves $P_{\mathrm{w},+}$ $\left(P_{\mathrm{w},-}\right)$, and the total energy density includes wave terms as well $e_{\mathrm{w},+}\left(e_{\mathrm{w},-}\right)$, see Ko 
(1992), Thomas and Pfrommer (2019) for a complete derivation of the new system of equations. This system is restricted to the quasi-linear theory framework. Hence, coupled with a MHD code the amplitude of self-generated waves has to be small with respect to the amplitude of the background magnetic field. The four-fluid system has been numerically solved by Thomas and Pfrommer (2019) using an explicit scheme separating the CR and wave fluid equations.

The interest of this approach resides in the more accurate calculation of the CR streaming speed given by Eq. (88) as well as the CR diffusion coefficient in space and energy, both dependent on the amplitude of scattering frequencies $v_{ \pm}$off forward and backward waves. In particular, it is possible to account for a fluid description of a stochastic acceleration term in the CR fluid energy density equation. This term has the form (Thomas and Pfrommer 2019)

$$
4 \frac{\bar{v}_{+} \bar{v}_{-}}{\bar{v}_{+}+\bar{v}_{-}} \frac{v_{\mathrm{a}}^{2}}{c^{2}}\left(e_{\mathrm{CR}}+P_{\mathrm{CR}}\right),
$$

where $\bar{v}$ is the momentum-averaged scattering frequency.

Other numerical strategies Pfrommer et al. (2006) and Enßlin et al. (2007) develop a method based on a smooth particle hydrodynamics (SPH) approach. In SPH fluid dynamics is treated using Lagrangian particles. To each particle is attached relevant fluid properties (e.g. density, pressure ...) calculated using a SPH kernel dependent on a smoothing length (Monaghan 1992). The CR distribution follows a power-law distribution whose normalization and power-law index vary under the effect of adiabatic gas variation (compression/expansion) as a function of the gas density (itself calculated in the SPH code).

\subsubsection{Numerical tests}

Aside the standard numerical tests for HD and MHD codes described in Sect. 5.2.8, we detail here some specific setups aiming at testing the transport (either passive or active) of CRs.

Hanasz and Lesch (2003) propose a series of tests of the CR flux term in Eq. (83) using the PIERNIK code (Hanasz et al. 2010). A first, straightforward test is 1D diffusion of CRs along the background magnetic field directed in one direction of the Cartesian grid, setting the perpendicular diffusion to zero, and no CR backreaction (turning off the CR pressure gradient), in a static medium. Diffusion can also be tested along an inclined magnetic field with contributions of the different directions to the CR flux. Figure 34 shows the profile of the CR energy density along and perpendicular to the ellipsoid solution of the propagation of an initial spheroidal distribution in 3D (see Fig. 2 in Hanasz and Lesch 2003).

The authors propose also the same test but now turning on the effect of CR pressure gradient. Figure 35 shows two plots: CR energy density and magnetic field lines (left), gas density and velocity field lines (right). It can be seen that the gas accelerates up to a few $\mathrm{km} / \mathrm{s}$ under the effect of a strong CR gradient preferentially along the background magnetic field line. This gas motion leads to a drift of CRs along the magnetic field. 


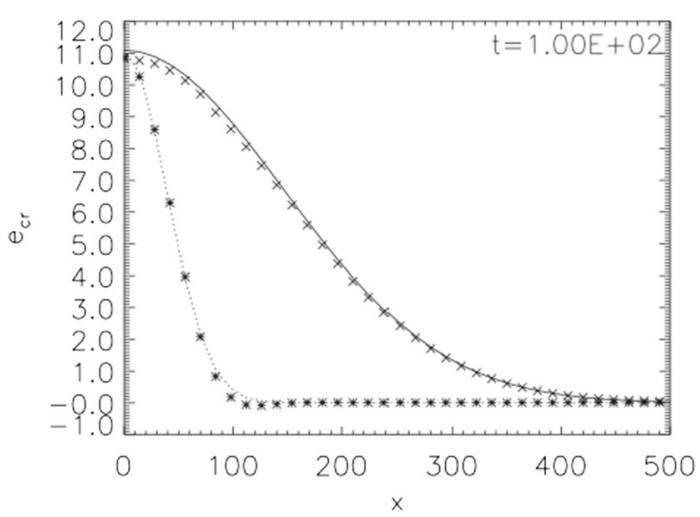

Fig. 34 CR propagation along an inclined magnetic field. The two curves show cuts of the ellipsoid solution at a given time along the major axis (crosses) and along the minor axis (asterisks). The solid and dotted lines represent the analytical solutions corresponding cuts of the fitted 2D Gaussian profile. CR diffusion is treated using an explicit scheme with $D_{\|}=100$ (in units of $\mathrm{pc}^{2} \mathrm{Myr}^{-1}$ and $D_{\perp}=0$. Image reproduced from Hanasz and Lesch (2003), copyright by ESO
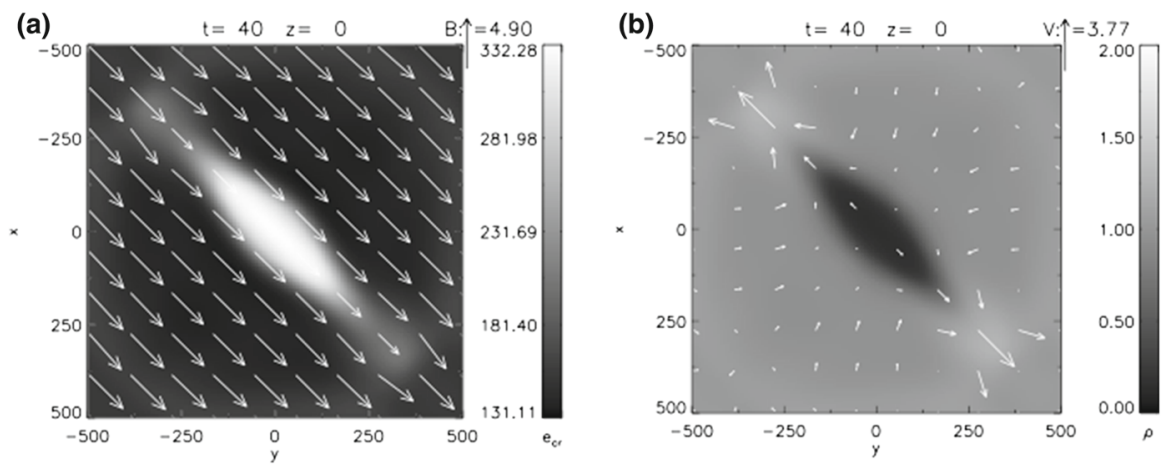

Fig. 35 CR propagation along an inclined magnetic field in the case CR backreaction is active. Equipartition between gas, magnetic field and background CR pressures is assumed. An over pressure of a factor 100 in CR is injected at the center at the start of the simulation. The diffusion coefficients are: $D_{\|}=100$ and $D_{\perp}=4$ (in units of $\mathrm{pc}^{2} \mathrm{Myr}^{-1}$ ). Left: CR pressure map and magnetic field lines. Right: gas density map and velocity vectors. Image reproduced with permission from Hanasz and Lesch (2003), copyright by ESO

Perpendicular diffusion imposes a broadening of the CR profile perpendicular to the magnetic field lines. Similar tests are proposed in Snodin et al. (2006) (see their Fig. 5).

A second type of tests used in the context of CR acceleration at SNR blast waves involves Sod shock-tube simulations (restricted to HD). Pfrommer et al. (2017) (see also Salem and Bryan 2014) derive an analytical solution of the shock-tube problem including a CR gas. Figure 36 shows a solution of a 1D Riemann shock tube problem. Three cases are shown: on the left the solutions for a shock propagating in a composite gas of thermal plasma and CRs but without any CR acceleration, in the middle the same case but now including CR acceleration, on the right a shock propagating in the thermal gas only but with CR acceleration. Butsky and Quinn (2018) propose a 1D Brio-Wu shock-tube test (hence in MHD). 

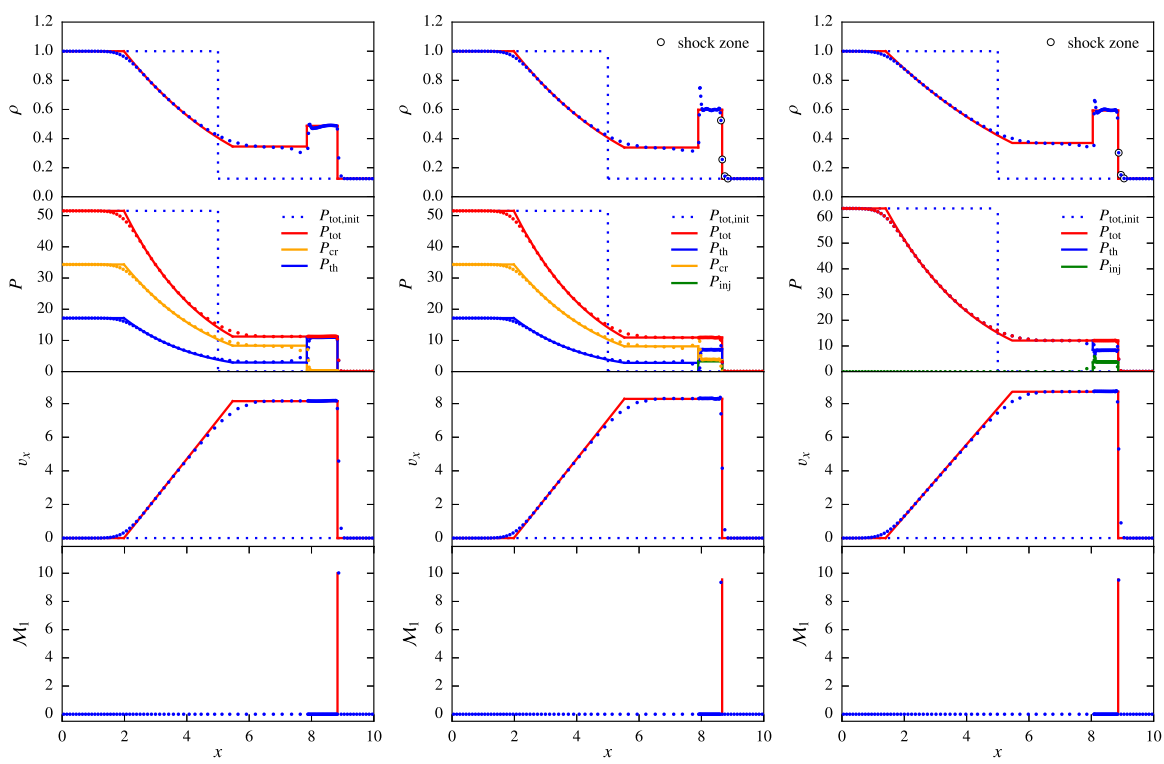

Fig. 36 1D shock-tube test problems including CRs. From top to bottom are shown: the gas mass density, gas pressure, gas velocity and shock Mach number. Left: shock propagation in a composite gas including CRs but without the effect of CR acceleration. Middle: same as the left case but now including CR acceleration. Right: same as the middle case but without background CRs. Solid lines: analytical solutions, dotted lines: numerical solutions. The different pressures are: gas (blue), CRs (orange), total (red), the pressure in the $\mathrm{CR}$ injected at the shock front is in green. In the left and middle cases the initial CR pressure is two times the gas pressure on the left part and equal to it on the right part. Image reproduced with permission from Pfrommer et al. (2017), copyright by the authors

\subsubsection{Cosmic ray physics using a bi-fluid approach}

We illustrate here the ability and the limits of the bi-fluid approach in treating three important aspects of CR physics: CR acceleration at shocks, CR-driven winds and $\mathrm{CR}$-induced magnetic production in the Galaxy.

Cosmic ray acceleration at shocks After the derivation of the test-particle solution produced by DSA (see Sect. 2.2), it appeared that the pressure carried by the high energy CRs is large enough to modify the shock structure and hence the Fermi acceleration process itself. Drury and Voelk (1981), Axford et al. (1977) derived self-consistent fluid solutions including the CR pressure in the Rankine-Hugoniot conditions using a bi-fluid model. They find that the non-linear model has up to three stationary shock solutions when the adiabatic indices of the gas and CR are $\gamma_{\mathrm{ad}}=5 / 3$ and $\gamma_{\mathrm{c}}=4 / 3$ respectively. The selection among these solution depends on the injection efficiency (Malkov 1997b). Saito et al. (2013) ${ }^{46}$ discuss the time-dependent stability of these solutions and confirm that the high- and low-efficiency solutions are stable against large-amplitude perturbations, while the intermediate one is not. At high-injection

\footnotetext{
46 Section 2 of Saito et al. (2013) provides a short updated review of the main assumptions and issues of the bi-fluid model.
} 
efficiency and high-shock Mach numbers the shock may be completely smoothed in the two-fluid approach. Malkov and Drury (2001), Malkov (1997a) show that a subshock must always exist in a kinetic model and hence that these smooth solutions are an artefact of the model because the maximum CR energy is always finite.

Acknowledging these issues, the main interest of the bi-fluid approach to investigate CR back-reaction resides in its simplicity and also in the fact that this formalism can be easily combined with a MHD model (Webb et al. 1986; Jun et al. 1994; Frank et al. 1995) and used to follow CR-MHD fluid non-linear dynamics with respect to the shock obliquity. The bi-fluid model also allows to combine effects of CRs and radiation (Wagner et al. 2007). It is particularly useful to diagnose the profile of $\mathrm{H}_{\alpha}$ lines in Balmer-dominated shocks occurring while a SNR propagate in a partially ionized medium (Wagner et al. 2009). However, only a kinetic calculation permits to follow the energy dependence of the particle distribution and the instabilities particles may generate. Non-linear kinetic models have since superseded the bi-fluid approach in this context. Numerical non-linear kinetic models are presented in Sects. 5.4 and 5.5 and semi-analytic models in Sect. 5.7.

CR driven winds CRs by the interplay of the generation of waves for instance by the streaming instability can convert a part of the CR bulk momentum into fluid momentum and hence drive winds (Ipavich 1975). Breitschwerdt et al. (1991), Breitschwerdt et al. (1993) investigate both analytically and numerically the launch of CR-driven winds in the framework of the bi-fluid model. Actually as their model includes also self-generated waves it proposes a three-fluid description. The authors use the flux tube approximation where stationary MHD equations are solved along the outwardlydirected magnetic field lines. The flux tube has a variable area cross section $A(z), z$ marking the height above the galactic disk. Solutions, especially the sonic point of the MHD system, are searched for as in the case of the solar wind. The numerical solutions start from the calculation of the fluid speed gradient at the critical point and then are propagated out- and downward to match the inner and outer boundaries. Stationary CR-driven outflows are obtained if the inter galactic gas pressure is low enough. Zirakashvili et al. (1996) extend this work by including galactic rotation. They show that the wind forces the gas in the halo to corotate with the galactic disk up to a distance of a few kpc. The waves generated by the CRs also contribute to heat the halo. Everett et al. (2008) adopted a similar modelling as in previous works although with different boundary condition assumptions. They find that thermal and CR pressures are equally important to drive the wind. Their solutions are found to be consistent with an injection of CRs by SNe at a standard rate although with a bit high efficiency. Uhlig et al. (2012) carry SPH simulations including a treatment of adiabatic gain/loss of the CR gas and the physics of streaming. Pakmor et al. (2016), Ruszkowski et al. (2017) add the effect of anisotropic diffusion coefficients in the disk in a bi-fluid formalism. All these works show that CRs have a systematic negative feedback impact over the star formation rate in our Galaxy while they can launch powerful winds from the galactic disk.

Galactic magnetic dynamo Dynamo is likely at the origin of large scale magnetic fields generated in the Galaxy (see Kulsrud 1999 and Sect. 6 in Brandenburg 2018). 
Bi-fluid models have been widely used to investigate the Parker or buoyancy instability thought to participate fast dynamo processes in spiral galaxies (Parker 1992; Hanasz and Lesch 1993). CRs produce an inflation of magnetic loops anchored in the galactic disk that induces magnetic reconnection and disconnects disk and halo magnetic fields. The closed loops in the halo and the anchored loops in the disk can be subject to cyclonic rotation at the origin of an $\alpha \Omega$ dynamo process, the $\alpha$ effect resulting from the Parker instability. Notice that CRs can provoke $\alpha$ dynamo because of their current, strong enough to trigger the non-resonant streaming instability (Beresnyak and Li 2014).

A numerical investigation of the Parker instability using a bi-fluid model has been proposed by Hanasz and Lesch (2000) using a flux-tube approximation. In that approximation CR are injected from a SNR at its lifetime end in the flux tube composed of the magnetic field lines threading the SNR. CRs are injected with an over-pressure of $\sim 30$ with respect to their background pressure and then inflate the flux tube in the vertical direction (with respect to the disk), eventually leading to a flux-tube explosion in a runaway process and ultimately to the production of galactic winds. ${ }^{47}$ The authors were able to find $\alpha$ coefficients large enough to ensure efficient Parker instability including magnetic field back-reaction. Ryu et al. (2003) investigate the growth rate of the Parker instability as function of the CR diffusion coefficient and find that the growth rate decreases with the parallel coefficient whereas the perpendicular coefficient has no strong impact. Kuwabara et al. (2004) conducte a 2D analysis of the Parker and explosion instabilities. Later Hanasz et al. (2004) and Hanasz et al. (2009) propose 3D simulations of the Parker instability using the PIERNIK code in a 3D box and in global Galactic geometry respectively. The global simulations show the growth of the magnetic field strength saturates at about $t=4 \mathrm{Gyr}$, reaching values of 3-5 $\mu \mathrm{G}$ in the disk. The magnetic field, initially randomly oriented, shows at the end of the simulation a toroidal component forming a spiral structure with reversals in the plane of the disk.

\subsection{The fluid-kinetic framework}

Solving the Fokker-Planck equation (FPE) is usually a very demanding task for computer simulations (see Sect. 3.6). Then, coupling a FPE with a MHD code has only been scarcely attempted yet. In this section we discuss approaches developed to couple a fluid model (HD or MHD) and a kinetic model (Vlasov or Fokker-Planck or approximations of these), appropriate to describe respectively the shock and the particles in the context of CR acceleration at shocks. In Sect. 5.4.1 we discuss methods that couple (M)HD equations and the diffusion-convection equation. In Sect. 5.4.2 we present a different approach that relies on a more general Vlasov-Fokker-Planck equation for description of the evolution of the distribution function $f(\mathbf{p})$.

\footnotetext{
47 Figure 1 in Lesch and Hanasz (2003) lists a series of physical processes which result from the Parker instability.
} 


\subsubsection{Coupling hydro and diffusion-convection equations}

Works reviewed in this section model separately the evolution of the astrophysical flow (e.g., the blast wave of a supernova remnant, or a cosmological structure formation shock), using HD or MHD equations (see Sect. 5.1), and the acceleration of energetic particles, using the diffusion-convection equation, that is the angle-averaged FokkerPlanck equation (see Sect. 3.6) of the form

$$
\frac{\partial f}{\partial t}+\frac{\partial}{\partial x}(u f)=\frac{\partial}{\partial x}\left(D \frac{\partial f}{\partial x}\right)+\frac{1}{3 p^{2}} \frac{\partial p^{3} f}{\partial p} \frac{\partial u}{\partial x},
$$

which includes advection terms in space and in momentum, and a diffusion term in space (we are here restricting ourselves to first order acceleration, and so have not included diffusion in momentum). When written in a conservative form the spatial advection term may be included in the hydrodynamical solver, using the operatorsplitting technique. The right-hand side is commonly solved with Finite Differences methods (FDM), using the techniques presented in Sect. 3.6. The diffusion term is the most difficult to treat. As stressed before, explicit schemes suffer from the constraint due to a CFL condition that is quadratic in the resolution $\Delta t<\Delta x^{2} / 2 D$, and which for physically-motivated values of the diffusion coefficient imposes time-steps that are much shorter than the hydrodynamic time-step. As in Sect. 5.3, this leads to the use of implicit or semi-implicit schemes, or possibly accelerated explicit schemes. The diffusion coefficient $D$, although it should in principle be computed from $f$ itself, is commonly prescribed as a function of $p$, usually as a power-law $D(p) \propto p^{\alpha}$, with optionally a dependence on position $x$ (the Bohm coefficient reduces to such a form with $\alpha=2$ in the non-relativistic regime and $\alpha=1$ in the relativistic regime). Compared to the bi-fluid approach of previous Sect. 5.3, the methods presented in this section aim at describing the spectrum of particles $f(p)$, using various approximations and techniques to handle the large range of scales to be resolved.

The piecewise power-law method This method was introduced by Jones et al. (1999), Tregillis et al. (2001) to investigate the time evolution of relativistic synchrotron-emitting electrons in radio galaxies. ${ }^{48}$ The basic idea is to approximate the electron distribution $f(p)$ as a piecewise power-law with $N$ bins in momentum, $f(p)=\sum_{\mathrm{i}} f_{0, i}\left(p / p_{\mathrm{i}}\right)^{\alpha_{\mathrm{i}}}$ with $i \in[1, N]$ (on a fixed, logarithmic momentum grid), and to calculate $f_{0, i}, \alpha_{\mathrm{i}}$ as function of the position in the simulation space (here a jet). The number of relativistic electrons in the bin $\mathrm{i}$ is $n_{\mathrm{i}}=\int_{p_{\mathrm{i}}}^{p_{\mathrm{i}+1}} f(p) 4 \pi p^{2} d p$, which can be normalized to the grid mass density of the gas. We note $b_{\mathrm{i}}=n_{\mathrm{i}} / \rho$. At shocks, the standard DSA theory in the test-particle limit is applied (see Sect. 2.2.1), the index is fixed to $\alpha=3 r /(r-1)$ where $r$ is the shock compression ratio evaluated

\footnotetext{
48 An alternative approach was proposed by Micono et al. (1999) where a set of Lagrangian test particles, a set of relativistic electrons is followed along a jet including acceleration and losses. Note that simulations combining mixed Lagrangian (for energetic particles) and Eulerian (for the magnetized fluid) coordinates have also been developed in the framework of relativistic flows by Vaidya et al. (2018). The authors solve the diffusion-convection equation for relativistic electrons transported in a relativistic flow calculated using a relativistic version of the PLUTO code.
} 
from the shock Mach number. At shocks $b=\sum_{\mathrm{i}} b_{\mathrm{i}}$ is calculated solving a simple equation $d b / d t=Q_{\text {inj }} / \rho$ where $Q_{\text {inj }}=\epsilon u_{\mathrm{sh}, \mathrm{L}} / \mu_{\mathrm{e}} m_{\mathrm{p}}$. Here $u_{\mathrm{sh}, \mathrm{L}}$ is the Lagrangian shock speed, $\mu_{\mathrm{e}}$ the electron mean mass, $m_{\mathrm{p}}$ the proton mass. The parameter $\epsilon$ is the fraction of thermal electrons injected as non-thermal particles. Away from shocks, the parameters $b_{\mathrm{i}}$ and $\alpha_{\mathrm{i}}=3-\left(b_{\mathrm{i}+1} / b_{\mathrm{i}}\right) / \Delta \ln (p)_{\mathrm{i}}$ evolve accounting for adiabatic and radiative losses (see Miniati 2001).

Adiabatic losses, which produce a shift in energy to lower energy, are of particular importance for CR-MHD systems discussed in Sect. 5.3. These systems can be extended to account for more than one CR population. This extension is reflected in the choice of the mean diffusion coefficient corresponding to a particular CR energy range of a given population. This effect can be treated with the piecewise power-law method (Miniati 2001; Girichidis et al. 2014). The adiabatic loss rate is $d \ln (p) / d t=\left(\gamma_{\mathrm{CR}}-1\right) \nabla . \mathbf{u}$. Here the adiabatic CR index $\gamma_{\mathrm{CR}}$ changes depending on the CR energy, from $5 / 3$ in the non-relativisitc case to $4 / 3$ in the relativistic case. The CR density can be computed from the relation $n_{\mathrm{i}}=E_{\mathrm{i}} /\left\langle E_{\mathrm{i}}\right\rangle$, where $E_{\mathrm{i}}$ is the CR energy in the ith bin, and the average energy per CR particle in bin $\mathrm{i}$ $\left\langle E_{\mathrm{i}}\right\rangle=\int_{p_{i}}^{p_{i+1}} E(p) p^{2} d p / \int_{p_{i}}^{p_{i+1}} p^{2} d p=e_{\mathrm{CR}, \mathrm{i}} / n_{\mathrm{i}}$ does not depend on $f$. The $\mathrm{CR}$ energy density in the bin i can then be updated accounting for the loss term

$$
e_{\mathrm{CR}, \mathrm{i}}^{n+1}=e_{\mathrm{CR}, \mathrm{i}}^{n}+\Delta t\left(\Phi_{\mathrm{i}-1 / 2}-\Phi_{\mathrm{i}+1 / 2}\right)
$$

where the energy flux is $\Phi_{i+1 / 2}=4 \pi / \Delta t \int_{t}^{t+\Delta t} b(E) E p^{2} f(p)_{i+1 / 2}$. The final energy $E(t+\Delta t)$ is expressed in terms of the adiabatic loss term and $\Delta t$ and has to be in the interval [ $\left.E_{i-1 / 2}, E_{i+1 / 2}\right]$, otherwise a sub-cycling is required (see Girichidis et al. (2014) for further details).

Including backreaction effects In efficient DSA the evolution of the shock and of the particles are non-linearly coupled (NLDSA). In the hydro-kinetic approach the connection between the fluid and the particles is made via extra terms in the equations, that model the injection and the back-reaction of particles. First, a source term is required in the diffusion-convection equation at the shock front, that depends on the shock jump conditions, and a corresponding loss term must be added in the set of hydrodynamic equations to ensure energy conservation (and in principle conservation of mass, but the inertia of energetic particles is normally negligible). Secondly, similar to the bi-fluid approach, one has to take into account the pressure of particles $P_{\mathrm{CR}}$ given by Eq. (9). The force $-\nabla P_{\mathrm{CR}}$ exerted by the particles on the flow is added as a source term to the equation of conservation of momentum, and the corresponding work $-\mathbf{u} . \nabla P_{\mathrm{CR}}$ is added as a source term to the equation of conservation of energy. At this level of modeling it is the gradient of particle pressure upstream of the shock that will cause the appearance of the shock precursor, ${ }^{49}$ which in turn will produce concave particle spectra.

The first investigations of time-dependent NLDSA using this approach were performed by Falle and Giddings (1987) and Bell (1987). Knowing that the canonical result for $f(p)$ is a power-law of index $s=4$, Falle and Giddings (1987) work with

\footnotetext{
49 At the PIC/hybrid level of modelling of Sect. 3.1, this is done via the Lorentz force.
} 
the quantity $g(p)=p^{4} f(p)$, and since the momentum gain is proportional to the current momentum they replace $p$ with the quantity $y=\ln p$. Equation (93) is then rewritten as

$$
\frac{\partial g}{\partial t}+\frac{\partial(u g)}{\partial x}=\frac{\partial}{\partial x}\left(D \frac{\partial g}{\partial x}\right)+\frac{1}{3}\left(\frac{\partial g}{\partial y}-g\right) \frac{\partial u}{\partial x} .
$$

These early works assumed a small dependence of the diffusion coefficient on $p$, using resp. $\alpha=1 / 4$ and $\alpha=1 / 2$, because of numerical limitations. As already noted in Sect. 2.2, it is the diffusion coefficient that sets the spatial and temporal scales of the simulation. The spatial scales range from the microscopic scale where the particles decouple from the fluid (of the order of a few thermal gyration lengths) to macroscopic scales (like the radius of the supernova remnant). The resolution of the numerical grid is dictated by the diffusion of the lowest energy particles, whereas the size of the grid is dictated by the diffusion of the highest energy particles. The ratio $D\left(p_{\max }\right) / D\left(p_{\min }\right)$, and thus the number of cells, may exceed ten orders of magnitude if $D(p) \propto p$, which is too demanding in terms of memory requirements and computing time. Fortunately we need very high resolution only around the shock, since low energy particles cannot travel far away from the shock. More generally, particles of a given momentum $p$ require a certain spatial resolution over a certain extent around the shock. This leads to the implementation of adaptive mesh refinement (AMR), reviewed in Sect. 5.2.5. In the context of DSA simulations, this technique was pioneered by Duffy (1992), to be able to use the true Bohm scaling $D(p) \propto p v$, and later used by Kang et al. (2001) and by Ferrand et al. (2008). The latter authors also parallelized the scheme in the $p$-direction, and studied repeated acceleration by multiple shocks. Ferrand et al. (2014a) use a different diffusion coefficient, proposed for perpendicular shocks, while DSA simulations are usually focused on the case of parallel shocks.

The hydro-kinetic approach was mostly developed by the team of Kang and Jones and collaborators with their code CRASH, starting from Kang and Jones (1991), with a number of publications produced until this date. An example of results can be seen in Fig. 43, compared with two other methods discussed elsewhere in this review. Some important numerical developments include the following. Gieseler et al. (2000) introduced an injection scheme based on the thermal leakage model. Kang et al. (2001) implemented a grid-based AMR scheme, as well as sub-zone shock tracking, in order to address realistic diffusion coefficients at a manageable computational cost. Jones and Kang (2005) applied a Coarse-Grained Finite Momentum-Volume Scheme (CGFMV), an extension of the piecewise power-law method introduced by Jones et al. (1999) already presented above. The basic idea is to lower the numerical resolution in momentum (down to as few as two to three bins per decade), but prescribe the shape of the spectrum in each bin so as to maintain reasonable accuracy; the numerical spectrum is then no longer a piecewise constant function but a piecewise linear function. All the works cited so far were restricted to slab geometry, Kang and Jones (2006) simulated shocks in spherical geometry-still effectively one-dimensional, under the assumption of spherical symmetry-in order to study CR feedback at SNR shocks. For this the authors employed a frame comoving with the outer shock, which was found to lower the convergence requirements. Following the formalism of cosmology, they use coordinate $\tilde{r}=r / a$ where $a(t)$ is the expansion factor, with expansion rate obtained from the 
measured shock speed in the Cartesian grid. Then density and pressure (or energy density) are re-scaled as $\tilde{\rho}=\rho a^{3}$ and $\tilde{P}=P a^{3}$ (the time variable is left unchanged). For quasi-parallel plane shocks, Kang and Jones (2007); Kang et al. (2009) found a selfsimilar evolution and proposed analytic forms for the solutions during that stage. All these improvements allowed the team to investigate the time evolution of CR-modified shocks and particle spectra, and eventually compute the non-thermal radiation from the accelerated particles in SNRs (Edmon et al. 2011; Kang et al. 2012). The latest developments are the inclusion of prescriptions for magnetic field amplification (MFA, see Sect. 2.2.4) and of the effects of the Alfvénic drift (see Eq. (88)) by Kang (2012), Kang (2013), Kang et al. (2013), with a formalism similar to the approaches discussed later in Sect. 5.7.2.

Other approaches in spherical geometry To account for the spatially-dependent diffusion of energetic particles, an alternative approach to AMR is to perform a change of variables, as done by Ptuskin et al. (2010); Zirakashvili and Ptuskin (2012) and by Telezhinsky et al. (2012b, a). These simulations are performed in spherically symmetric geometry, and notably include the reverse shock and the contact discontinuity as well as the forward shock (located at radius $R_{b}, R_{c}$, and $R_{f}$ respectively).

In Ptuskin et al. (2010), Zirakashvili and Ptuskin (2012) the radius $r$ is replaced by $r / R_{b}$ for $r<R_{b}$ and $r / R_{f}$ for $r>R_{f}$ (unshocked medium), and by $(r-$ $\left.R_{c}\right) /\left(R_{c}-R_{b}\right)$ for $R_{b}<r<R_{c}$ and $\left(r-R_{c}\right) /\left(R_{f}-R_{c}\right)$ for $R_{c}<r<R_{f}$ (shocked region). Also time is replaced by a dimensionless parameter that scales as $\ln \left(R_{f}\right)$. The hydrodynamic equations are solved using FDM, separately in the upstream regions (using an implicit scheme since the flow is supersonic) and in the downstream regions (using an explicit scheme), and the three discontinuities between the different regions are moved manually in time. As in other methods, the diffusion-convection equation for particles is recast as a set of tri-diagonal equations. In these works the CR pressure is included in the HD equations, and the velocity used for particles includes the Alfvénic drift. An example of the results is shown in Fig. 37, for a fiducial SNR at age 1000 year.

In Telezhinsky et al. (2012b,a) the radius is first normalized to the outer shock radius: $x=r / R_{f}$ (a comoving coordinate, as in Kang and Jones 2006) and then transformed according to $(x-1)=\left(x_{*}-1\right)^{3}$, so that $d x / d x_{*}=3\left(x_{*}-1\right)^{2}$, with a uniform binning in new coordinate $x_{*}$. The transport equation for the particles is solved using an implicit FDM, while the HD equations are solved with the VH- 1 code. These simulations were made in the test-particle regime, they do not include the back-reaction of energetic particles on the hydrodynamics. Telezhinsky et al. (2012a) discuss the possible contribution to the SNR emission of CRs accelerated at the reverse shock w.r.t. those accelerated at the forward shock.

\subsubsection{Coupling MHD and Vlasov-Fokker-Planck equations}

Bell et al. (2011), Reville and Bell (2013) develop a method based on performing a spherical harmonics expansion of the distribution $f$ in momentum $\mathbf{p}$, using the KALOS code previously developed for simulations of laser-plasma interactions (Bell et al. 2006). The authors consider a $1 \mathrm{D}$ problem with a planar shock (with the normal to the shock front oriented in the $\mathrm{x}$ direction with $x<0$ the upstream medium) and keep the 

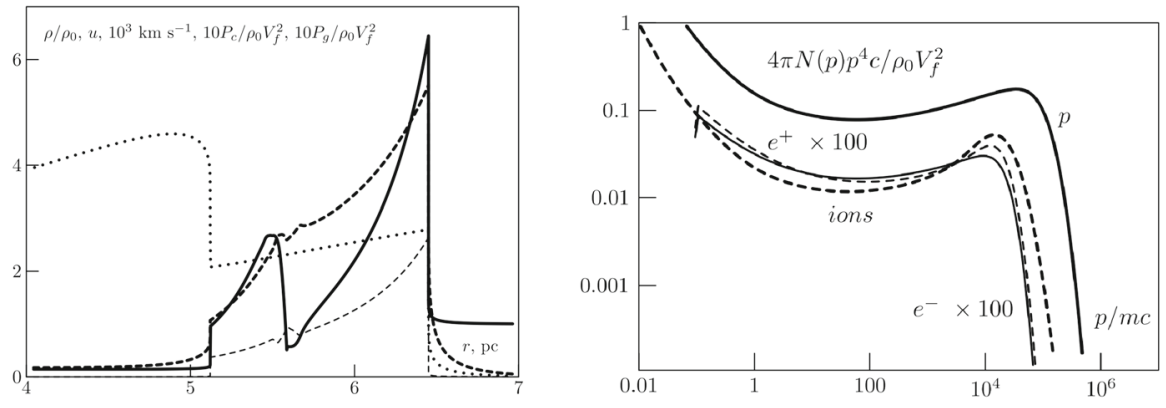

Fig. 37 Representative results from a hydro-kinetic simulation of a SNR in spherical geometry, at $t=$ 1000 year. The supernova has typical mass $1.4 M_{\odot}$ and energy $10^{51} \mathrm{erg}$, released in a uniform medium of density $0.1 \mathrm{~cm}^{-3}$, temperature $10^{4} \mathrm{~K}$, and magnetic field $5 \mu \mathrm{G}$. Left: radial dependencies of gas density (thick solid line), gas velocity (dotted line), CR pressure (thick dashed line), and gas pressure (dashed line). The reverse and forward shocks are visible as discontinuities in the hydro profiles. An extended CR precursor is visible ahead of each shock. Right: spectra of accelerated particles: protons at the forward shock (thick solid line), ions at the reverse shock (thick dashed line), electrons at the forward shock $(\times 100$, thin solid line), and positrons at the reverse shock $(\times 100$, thin dashed line) present from radioactive decay in the ejecta. Note the concavity of the spectra, plotted as $p^{4} f(p)$. Image reproduced with permission from Zirakashvili and Ptuskin (2012), copyright by Elsevier

information about the momentum vector $\mathbf{p}$. They solve the following Vlasov-FokkerPlanck (VFP) equation (Bell et al. 2011)

$$
\begin{aligned}
& \partial_{t} f(x, \mathbf{p}, t)+\left(v_{x}+u\right) \partial_{x} f-\partial_{x} u p_{x} \partial_{p_{x}} f-\frac{u}{c} \partial_{x} u p_{x} \partial_{p_{x}} f \\
& \quad+q \mathbf{v} \times \mathbf{B} \cdot \partial_{\mathbf{p}} f=\mathcal{C}(f),
\end{aligned}
$$

where $v$ and $u$ stand for the particle and the fluid speeds, and q is the particle charge. The particle distribution function is measured in the fluid rest-frame. $\mathbf{B}$ is the local magnetic field. The term $\mathcal{C}(f)$ is a collision term; in the collisionless plasmas investigated here this term is due to wave-particle interactions. It is written

$$
\mathcal{C}(f)=\partial_{\mu} v_{\mathrm{s}}\left(1-\mu^{2}\right) \partial_{\mu} f(x, \mathbf{p}, t)
$$

where $\mu=\cos (\theta)$ and $\nu_{\mathrm{s}}$ is a parameter to be scaled with respect to the particle gyrofrequency $\Omega=q B / \gamma m c$. In this model particle angular deflection is assumed to be small. This justifies the use of the term Fokker-Planck.

The particle distribution is then expanded into spherical harmonics as

$$
f(x, \mathbf{p}, t)=\sum_{l, m} f_{l}^{m}(x, p, t) P_{l}^{|m|}(\cos \theta) \exp (-i m \phi)
$$

where $l$ is a positive integer, $m$ is an integer between $-l$ and $+l, \theta$ is the particle momentum pitch-angle with respect to the shock normal, $\phi$ is the particle momentum azimuthal angle, and $P_{l}^{m}(\cos \theta)$ are the associated Legendre polynomials of order $l$. We have $f_{l}^{-m}=\left(f_{l}^{m}\right)^{*}$, the complex conjugate of $f_{l}^{m}$. By applying this expansion 
to the VFP equation, one obtains a hierarchy of equations for each component $f_{l}^{m}$, not reproduced here [see Eq. (2) of Bell et al. 2011]. Usually, as in shocks the particle distribution is close to isotropy due to efficient wave-particle scattering it is only necessary to retain the first few terms $f_{0}^{0}, f_{1}^{0}$, and $f_{1}^{1} \cdot f_{0}^{0}$ represents the isotropic part of the distribution function while $f_{1}^{0}$ represents the particle flux along the shock normal. $f_{1}^{1}$ complements the construction of the CR current. In the limit of a small ratio $u / c$ the system reduces to a simple system using the Chapman-Enskog expansion $f_{l}^{m} \sim(u / c)^{l} f_{0}^{0}$ (with $v \simeq c$ ). In the upstream medium

$$
\begin{aligned}
\frac{c}{3} \partial_{x} f_{0}^{1}+u \partial_{x} f_{0}^{0} & =0, \\
c \partial_{x} f_{0}^{0}+2 \Omega_{z} \operatorname{Re}\left(f_{1}^{1}\right) & =-v_{\mathrm{s}} f_{1}^{0}, \\
-i \Omega_{x} f_{1}^{1}-\frac{\Omega_{z}}{2} f_{1}^{0} & =-v_{\mathrm{s}} f_{1}^{1},
\end{aligned}
$$

while in the downstream medium the particle distribution is isotropic, so limited to the term $f_{0}^{0}$. The solution at the shock front can be obtained using the continuity of the particle distribution and its flux at the shock front, accounting for the frame transformation across the shock. Looking for a power-law solution $f \propto p^{-\gamma}$, at the leading order in $u / c$ one finds

$$
\gamma=3+3 \frac{u_{2}}{u_{\mathrm{sh}}-u_{2}} \frac{f_{0}^{0}(+\infty)}{f_{0}^{0}(x=0)}
$$

where $u_{2}$ is the fluid speed and $f_{0}^{0}(+\infty)$ the particle isotropic component far downstream. One recovers $\gamma=4$ for a strong unmodified shock.

Oblique shock solutions Bell et al. (2011) apply the above formalism to an oblique flow, where the magnetic field direction is given by the angle $\theta$ with respect to the shock normal. The procedure requires to start with a guess of the particle distribution at the shock front as a power-law. The index $\gamma$ is calculated iteratively with the full set of equations for $f_{l}^{m}$. These are solved using FDM techniques (see Sect. 3.6.1) with $f_{l}^{m}$ calculated at the cell centers for even $l$ and at the cell boundaries for odd $l$. The spatial resolution is finer close to the shock front and coarser at the edge of the simulation box. Non-linear CR backreaction is not considered in this work: the background magnetic field and fluid velocity are not modified by the CR pressure. The shock velocity profile is smoothed using a hyperbolic tangent profile of width $x_{\mathrm{s}}$ of $1 \%$ of the particle Larmor radius upstream. The shock index solutions show soft distributions at quasiperpendicular fast shocks. Such configurations can occur if strong magnetic field amplification occurs after the supernova shock breakout (Marcowith et al. 2018) or/and if the shock propagates in circumstellar medium with a spiral magnetic field (Bell 2008).

CR driven instabilities Reville and Bell (2013) and Bell et al. (2013) couple the VFP method described above to MHD solutions in order to calculate the CR pressure backreaction over the shock solutions. The authors adopt mixed coordinate frames (Skilling 
1975a) where the particle momenta are evaluated in the local fluid frame. It has the advantage of considerably simplifying the collision term on the r.h.s. of Eq. (96), which is parametrized by a scattering frequency $v_{\mathrm{s}}$. The spherical harmonic expansion is stopped at the first order and $f_{0}$ and $\mathbf{f}_{1}$ are used to evaluate the CR charge density and current to be re-injected into the ideal MHD equations (see Sect. 5.1.1). The vector $\mathbf{f}_{1}=f_{\mathrm{x}} \mathbf{e}_{\mathrm{x}}+f_{\mathrm{y}} \mathbf{e}_{\mathrm{y}}+f_{\mathrm{z}} \mathbf{e}_{\mathrm{z}}$, where $f_{\mathrm{x}}=f_{1}^{0}$, and the perpendicular components are $f_{\mathrm{y}}=-2 \mathcal{R} f_{1}^{1}$ and $f_{\mathrm{z}}=2 \mathcal{I} f_{1}^{1}$. The CR charge and current densities can be defined as

$$
\begin{aligned}
& \rho_{\mathrm{CR}}=4 \pi q \int f_{0}^{0} p^{2} d p \\
& J_{\mathrm{CR}}=\frac{4 \pi}{3} q \int\left[\begin{array}{c}
f_{1}^{0} \\
-2 \mathcal{R} f_{1}^{1} \\
2 \mathcal{I} f_{1}^{1}
\end{array}\right] p^{2} v d p .
\end{aligned}
$$

The current drives a Lorentz force $-\mathbf{J}_{\mathrm{CR}} / c \times \mathbf{B}$ and induces a plasma heating $-\mathbf{J}_{\mathrm{CR}} . \mathbf{E}$. (We will come back on CR-MHD coupling in Sect. 5.5.)

The numerical scheme relies on the following approximations:

1. The particle distribution follows a power-law.

2. A ratio $\nu_{\mathrm{s}} / \Omega_{\mathrm{s}}<1$ ensures the closing of the spherical harmonics expansion.

3. Another component $f_{\mathrm{LS}}$ is added to $f_{\mathrm{X}}$ in order to mimic the effect of a large scale CR component slowly varying over the simulation domain.

Spatially, the simulations are 3D and use periodic boundary conditions. The CFL condition requires some sub-cycling of the MHD step to account for the CR evolution.

In Fig. 38 we show the solution of the 3D VFP-MHD system at a particular time. The simulation has the following setup: a background gas density $n=0.1 \mathrm{~cm}^{-3}$, a background parallel magnetic field $B=47 \mu \mathrm{G}$, a shock speed $U_{\mathrm{sh}}=60,000 \mathrm{~km} \mathrm{~s}^{-1}$, the $\mathrm{CR}$ are injected at $100 \mathrm{TeV}$. The background field is oriented in the $\mathrm{z}$ direction. The simulation box has 5676 cells in this direction and 32 in the $\mathrm{x}$ and $\mathrm{y}$ directions. The CR current is initialized along z. Figure 38 clearly shows two CR populations (see panel c). A first one is escaping ahead of the shock and generating a current (see panel e). The return current in the plasma then triggers non-resonant streaming modes and magnetic field fluctuations which induce a confinement of a second population of CRs at the shock front. This population is accelerated via DSA. Inoue (2019) has adapted this procedure to the case of a SNR propagating in a molecular cloud. There TeV CRs are released first and produce through their current enough magnetic perturbations to confine GeV CRs around the shock front.

\subsection{Particle-in-cell-magnetohydrodynamics}

The approach presented in this section combines a MHD approximation necessary to derive the evolution of the background thermal plasma coupled to a PIC module necessary to calculate the trajectories of supra-thermal particles imposed by the Lorentz force. Contrary to standard PIC simulations, now the Lorentz force is calculated using the electro-magnetic field derived from the system of MHD equations rather than 

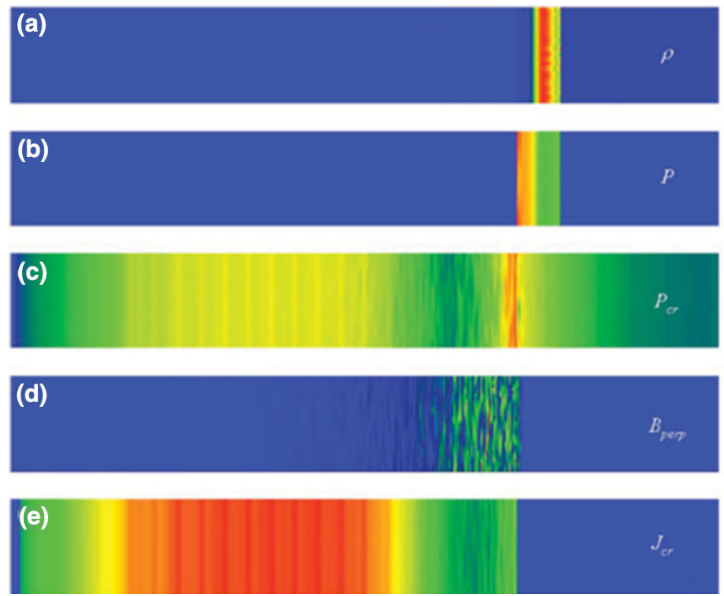

Fig. 38 2D slices of 3D simulation of the VFP-MHD system. From top to bottom: gas density, gas pressure, $\mathrm{CR}$ pressure, magnetic field component perpendicular to the shock normal, CR current. All quantities are obtained at a time of 1.3 year. The scales in the $\mathrm{z}$ direction are compressed by a factor 24 . Image reproduced with permission from Bell et al. (2013), copyright by the authors

Maxwell equations (Lucek and Bell 2000; Reville and Bell 2012). From the distribution of macro particles obtained by the PIC module it is now possible to derive the electric charge and current distribution associated with the supra-thermal particles and to re-inject these two quantities into the MHD equations. This modifies the dynamics of the background plasma. We also need to update the Ohm's law to account for the electric field associated with the energetic particles. Bai et al. (2015); van Marle et al. (2018); Mignone et al. (2018) are three recent works which propose the PIC-MHD approach adapted to the problem of CR in astrophysical flows incluing such a modified Ohm's law. They are based on three different MHD codes: respectively ATHENA, MPI- AMRVAC, and PLUTO.

The PIC-MHD model assumes global plasma electro-neutrality, i.e., $\sum_{\alpha} n_{\alpha} q_{\alpha}+$ $e\left(n_{\text {th,i }}-n_{\text {th,e }}\right)=0$, where the index $\alpha$ runs over the different supra-thermal population species: electrons, positrons, ions and $n_{\mathrm{th}, \mathrm{p} / \mathrm{e}}$ stands for the thermal background electron and ion densities (here we have assumed the plasma to be composed of protons only for simplicity). Non-thermal particles carry a current density $\mathbf{J}_{\mathrm{EP}}=\sum_{\alpha} n_{\alpha} q_{\alpha} \mathbf{u}_{\alpha}$ to be inserted into the Ampère's law. In the general Ohm's law Hall and electron pressure gradient terms can be safely neglected because they become important only at scales smaller than the ion inertial scale $c / \omega_{\text {pi }}$, see Bai et al. (2015). The modified Ohm's law can the be written as

$$
c \mathbf{E}=\left[(1-R) \mathbf{u}+R \mathbf{u}_{E P}\right] \times \mathbf{B},
$$

where

$$
R=\sum_{\alpha} \frac{n_{\alpha} q_{\alpha}}{n_{\mathrm{th}, \mathrm{e}} e}
$$


is a measure of the relative density of energetic particles (the ideal Ohm's law is recovered by setting $R=0$ ), and

$$
\mathbf{u}_{E P}=\frac{\sum_{\alpha} n_{\alpha} q_{\alpha} \mathbf{u}_{\alpha}}{\sum_{\alpha} n_{\alpha} q_{\alpha}}=\frac{\mathbf{J}_{\mathrm{EP}}}{n_{\mathrm{EP} e}}
$$

is the average velocity of the energetic particle population defined in terms of the energetic particles current $\mathbf{J}_{\mathrm{EP}}$ and density $n_{\mathrm{EP}}$. Energetic particles (or CRs) then induce a specific Hall effect. Even if $n_{\mathrm{EP}} / n_{\text {th,e }} \ll 1$ as it is the case for CRs, the average speed of the non-thermal particles may not be small compare to $\mathrm{c}$, so it has to be retained in the modified Ohm's law. The Lorentz force to be inserted as a source term in the Euler and energy conservation equations is now:

$$
\mathbf{F}_{\mathrm{EP}}=(1-R)\left(n_{\mathrm{EP}} e \mathbf{u} \times \mathbf{B}+\frac{\mathbf{J}_{\mathrm{EP}}}{c} \times \mathbf{B}\right) .
$$

The Lorentz equation which controls supra-thermal particle trajectories can be written as (for particle $\mathrm{j}$ of type $\alpha$ ):

$$
\frac{\partial \mathbf{p}_{\alpha, j}}{\partial t}=\frac{q_{\alpha}}{c}\left(\mathbf{u}_{\alpha, j}-R \mathbf{u}_{\mathrm{EP}}-(1-R) \mathbf{u}\right) \times \mathbf{B} .
$$

Again setting $R=0$ gives the standard form of the Lorentz equation. Notice now that the ensemble of energetic particles produce an electric field which modifies the trajectory of each energetic particle.

Energy conservation can be obtained by imposing within each cell the condition:

$$
\sum_{\alpha} \sum_{j} n_{\alpha} \mathbf{u}_{\alpha, j} \cdot \frac{\partial \mathbf{p}_{\alpha, j}}{\partial t}=\mathbf{F}_{\mathrm{EP}} \cdot \mathbf{u}_{\mathrm{EP}} .
$$

\subsubsection{Numerical schemes}

All works cited above use a Boris pusher to integrate energetic particle tracks with time (Birdsall and Langdon 1985). In order to reach second-order accuracy of the PIC-MHD scheme, Bai et al. (2015) (see also Mignone et al. 2018) introduce a predictor-corrector scheme to calculate the background plasma evolution under the effects of energetic particles. In the predictor (resp. corrector) part the source terms in the MHD equations are deduced from the location of individual particles to neighboring grid cell centers at the initial time (resp. at half the MHD timestep). The momentum and energy feedback are calculated at cell centers to derive new fluid solutions. This feedback calculation ensures second order accuracy. Aside the corner transport upwind (CTU) scheme (Colella 1990), Mignone et al. (2018) also introduce a second order Runge-Kutta time stepping method in the predictor-corrector scheme. van Marle et al. (2018) use a different strategy. Each component, i.e., the MHD fluid and the energetic particles, evolves over their own grid: the MHD grid is used as a base and the PIC grid is offset so that MHD cell centers stand as PIC cell corners. 


\subsubsection{Coupling MHD and PIC time steps}

In order to properly resolve each particle trajectory the MHD timestep deduced from the CFL condition has to verify $\Delta t r_{\mathrm{g}} \leq \zeta$, with $\zeta$ a constant to be adjusted and $r_{\mathrm{g}}$ the maximum gyroradius of the energetic particles. For instance in the case of a fixed grid, Bai et al. (2015) use $\zeta=0.3$ for shock problem studies. In the case of nonrelativistic MHD flows, the kinetic part evolves more rapidly than the MHD part. To treat this issue it is possible to impose some sub-cycling of the PIC step. Bai et al. (2015) typically use 10 sub-cycles. Mignone et al. (2018) improve this strategy and propose two sub-cycling schemes which recalculate the energetic particle induced Lorentz force at every sub-steps or at even sub-steps.

\subsubsection{Adaptive mesh refinement for the PIC module}

van Marle et al. (2018) have adopted an adaptive mesh refinement procedure for the PIC part, conversely Bai et al. (2015) use a fixed grid. The octree system of adaptive mesh refinement of the MPI- AMRVAC code is used but it involves an additional refinement condition on the energetic particles: if the number of energetic particles within a grid reaches $25 \%$ of a pre-set maximum the grid is no longer allowed to coarsen. If the number of particles reaches $80 \%$ of the maximum, the grid is refined (assuming it has not yet reached the maximum allowed refinement level). A supplementary condition is applied over the average gyroradius of the particles: if within the grid it becomes smaller than a pre-set number of times the size of the individual grid cells the grid is allowed to be refined. Hence, the particle gyro-radius is always resolved which is a necessary condition for a correct calculation of the source terms in the MHD equations.

A tricky aspect of mesh refinement appears when a particle is moving from one cell of a given level to another cell of a finer level. In that case it is essential to conserve charge and current. Then, the effective weight of the particle has to be increased by a factor equivalent to the reduction in effective volume. This effective weight has to be carefully calculated on the finer grid if a particle stands at the boundary of two grids at different levels. The physical extent where the weight is calculated is fixed by the coarser mesh, so the calculation of the particle weight may cover several rows (in a $2 \mathrm{D}$ view) of the finer grid (see Fig. 1 in van Marle et al. 2018).

\subsubsection{Numerical tests}

Bai et al. (2015) propose two tests of the PIC-MHD method. The first one follows the evolution in time of a test-particle in an uniform magnetic field, so it tests particle gyromotion. Different setups have been tested: non-relativistic and relativistic particles, background plasma moving perpendicularly to the background field. Figure 39 shows the time evolution of the particle energy in the co-moving plasma frame and the evolution of the particle position in the co-moving frame in the relativistic case. It can be seen that the Boris pusher conserves the energy perfectly in the case of no drift, and to better than $0.1 \%$ level in the case of a small drift (a bit better than in the non-relativistic case as the energy conservation degrades with respect to the relative speed of the drift with respect to the particle speed). The particle trajectory fits with the 

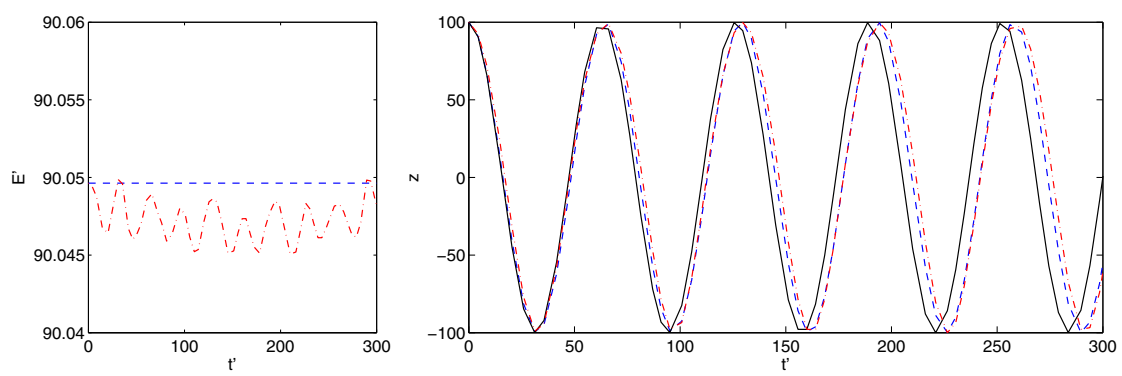

Fig. 39 Gyromotion test for a relativistic particle (with a Lorentz factor $\gamma=10$ ). The normalized variable time step is $\Delta t \Omega_{\mathrm{L}}=0.5 \pm 0.1$. Dashed blue lines: numerical solutions obtained in the case of a null drift velocity. Red dashed lines: numerical solutions obtained in the case of a drift velocity with strength $u_{\mathrm{d}}=U_{\mathrm{A}}$. Left panel: time evolution of the particle energy in the co-moving frame (indicated by a prime). Right panel: particle motion in the co-moving frame. The black curve is the analytical solution. Image reproduced with permission from Bai et al. (2015), copyright by AAS
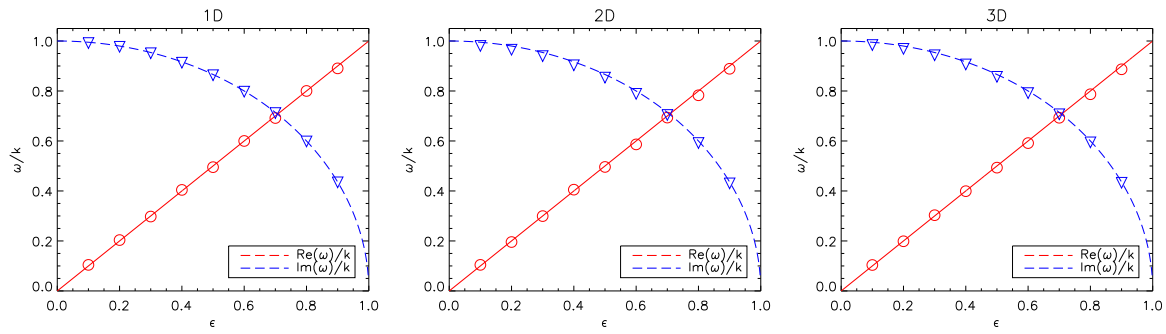

Fig. 40 Real and imaginary part of the non-resonant instability relation dispersion as a function of $\epsilon=$ $U_{\mathrm{A}} / U_{\mathrm{CR}}$. Solid lines: analytical solution. Diamond and circle: numerical solutions. Image reproduced with permission from Mignone et al. (2018), copyright by AAS

analytical solution well for long time evolution. Mignone et al. (2018) obtain similar results for this test. The authors propose another setup which tests the motion of a particle in a non-orthogonal electric and magnetic field. Energy conservation to $0.1 \%$ level is found for a mildly relativistic particle with respect to the analytical solution (see their Fig. 3).

A second setup concerns the test of the CR feedback. It captures the linear growth rate of the non-resonant (or Bell) instability (Bai et al. 2015; Mignone et al. 2018). In this setup, the background plasma is uniform and at rest, it is pervaded by a uniform magnetic field and a CR current is propagating along the background magnetic field with a speed $U_{\mathrm{CR}}$. The results are displayed in Fig. 40. The plots show the real and imaginary parts of the dispersion relation as a function of the ratio of the background Alfvén speed and the CR drift speed. A very good agreement between analytical (solid lines) and numerical (symbols) solutions is obtained.

Finally, Mignone et al. (2018) propose a setup to test the relative drift of the thermal gas and CRs. In this test, the two components drift in opposite direction perpendicularly to a background uniform magnetic field. This test is used to evaluate the performances of the predictor-corrector scheme and the impact of the number of CR sub-cyclings. 
The results show that the predictor step is mandatory in order to reproduce the analytical expected solution and to reach second order accuracy in time.

\subsubsection{Results on cosmic ray acceleration at non-relativistic shocks}

CR acceleration and CR driven instabilities at SNR shocks can be investigated with the help of the PIC-MHD method, as, in contrast to the case of hybrid techniques, now the PIC part only has to handle supra-thermal particles. This improves a lot particle statistics, which is necessary to properly reconstruct the CR charge and current.

Simulation setups Reville and Bell (2012) investigate the development of CR driven instabilities at shocks in the PIC-MHD framework generalizing the work of Lucek and Bell (2000) restricted to the resonant streaming instability. In this work the background plasma is at rest and pervaded by a uniform magnetic field augmented with a turbulent spectrum composed of modes in the perpendicular plane. CR are initialized with a mono-energetic distribution, drifting with respect to the background plasma. A total of 1024 particles per grid cell is used to ensure gyrotropy. The drift speed of CRs corresponds to a shock Alfvénic Mach number of initially $M_{\mathrm{A}}=10^{3}$. CRs are injected at an energy that allows the gyromotion to be resolved. The fraction of incoming kinetic energy imparted into CRs is $10^{-4}$.

Bai et al. (2015) also report on fast super-Alfvénic shock simulations. Their setup is as follows: a plasma flow is launched against a static, conductive wall (Mignone et al. (2018) proceed similarly). Quasi instantaneously after the collision a shock forms which propagates in the upstream medium with a speed $u_{\mathrm{sh}}>U_{\mathrm{A}, \mathrm{u}}$, where $U_{\mathrm{A}, \mathrm{u}}=B_{\mathrm{u}} / \sqrt{4 \pi \rho_{\mathrm{u}}}$ is the upstream Alfvén speed. The shock position is identified by evaluating the transversely-averaged fluid speed along the shock propagation direction (let say $x$ ) $u_{\mathrm{x}}$. This speed is further smoothed by a Gaussian kernel of typical size of 4 grid cells. Then the shock position is fixed at the location where $u_{\mathrm{x}}$ has decreased by $40 \%$. Supra-thermal particles are injected as CRs at a kinetic energy of $E_{\text {inj }}=10 E_{\text {sh }}$ at the shock position. The CR population injected is normalized to a small fraction of the incoming flux. Another important aspect is that the authors use an artificial light speed $\mathcal{C}$ different from $\mathrm{c}$ for CRs but still much larger than any fluid speed in the system. The Lorentz factor of species $\alpha$ of an individual CR is: $\gamma_{\mathrm{j}}=\mathcal{C} / \sqrt{\mathcal{C}^{2}-u_{, j}^{2}}$. This speed is inserted into the Lorentz equation. It has the advantage to be adapted to the investigation of different particle energy regimes (a large $\mathcal{C}$ is adapted to follow non- or mildlyrelativistic CRs and a small one is adapted to the relativistic regime). Bai et al. (2015) report on the production of non-thermal particle distribution consistent with the Fermi first order process, which is a strong indication that PIC-MHD methods can handle CR scattering off self-generated waves. van Marle et al. (2018) use a different shock generation procedure. The shock is set up from a configuration following the RankineHugoniot conditions. Particles are then injected following the same procedure as in the Bai et al work. Table 4 summarizes the setups of these different works.

Non-resonant CR driven instability studies and shock acceleration The main objective of these first studies is the onset of non-resonant (NR) streaming modes in the 
Table 4 Setups for shock acceleration studies

\begin{tabular}{lllll}
\hline Authors & $\begin{array}{l}\text { Resolution } \\
\left(r_{\mathrm{g}}\right)\end{array}$ & $\begin{array}{l}t_{\max } \\
\left(\omega_{\mathrm{c}}^{-1}\right)\end{array}$ & $M_{\mathrm{A}}$ & $\begin{array}{l}\theta_{\mathrm{B}} \\
\left({ }^{\circ}\right)\end{array}$ \\
\hline Bai et al. (2015) & $1.210^{5} \times 3000$ & $310^{3}$ & 30 & 0 \\
& $3.8910^{5} \times 4800$ & $1.210^{4}$ & 30 & 0 \\
van Marle et al. (2018) & $240 \times 30$ & $210^{3}$ & $3-30-300$ & $0-70-90$ \\
Mignone et al. (2018) & $1.210^{5} \times 3000$ & $310^{3}$ & 30 & 0 \\
& $3.8410^{5} \times 4800$ & $1.110^{4}$ & 30 & 0 \\
\hline
\end{tabular}

All simulations have been done in 2D space configuration. The spatial resolution for van Marle et al. (2018) is the basic one, the simulations also include four levels of refinement. The maximum time $t_{\max }$ is in units of $\omega_{\mathrm{c}}^{-1}$. The last column displays the shock magnetic obliquity. The second row for Bai et al. (2015) and Mignone et al. (2018) shows the setup for relativistic runs with a reduced light speed $\mathcal{C}$

context of strong shocks. ${ }^{50}$ All these studies have considered the case of parallel superAlfvénic shocks and conclude that indeed CRs trigger a streaming instability in the non-resonant regime (see Fig. 41). The theoretical linear growth timescale is recovered by the simulations. A Fourier analysis shows the destabilization of the main NR modes in the upstream medium at a scale fixed by the CR current (see Fig. 42). The interest of the PIC-MHD method with respect to previous MHD simulations (e.g., Bell 2004; Zirakashvili and Ptuskin 2008) is that it accounts for the time evolution of the CR-MHD including CR back reaction. A clear transfer to smaller wave numbers (hence large scales) can be noticed on Fig. 42. The back-reaction of CR also induces a strong corrugation of the shock front as can be seen on Fig. 41. The corrugation is so strong that it becomes difficult to identify the position of the shock front. Longer term simulations in $3 \mathrm{D}$ are required to investigate the transition from a non-relativistic to relativistic regime and to capture the propagation of the CRs in the self-generated turbulence (van Marle et al. 2019).

van Marle et al. (2018) have also considered an oblique shock configuration with a magnetic obliquity angle of $70^{\circ}$ with respect to the shock normal. The authors find that particle acceleration is delayed but still present. Particle acceleration proceeds in two steps in this configuration. First SDA occurs that injects a population of charged CRs upstream, which trigger non-resonant streaming modes parallel to the background magnetic field, which in turn perturb the magnetic field downstream. This perturbation leads to a corrugation of the shock front, which changes the orientation of the magnetic field and ultimately allows particles to be injected in a parallel configuration. Here again long-term 3D evolution are necessary to investigate the dynamics of particle acceleration and transport. At perpendicular (super-luminal) shocks the electromotive electric field cannot be compensated upstream and the authors do not observe any particle acceleration. The results on the oblique shock configuration have triggered some discussions. The main argument against is (Caprioli and Spitkovsky 2014a; Caprioli et al. 2018) that at high obliquity CR injection is not efficient because incoming

\footnotetext{
50 Reville and Bell (2012) discuss a filamentation instability generated by the CRs drifting ahead the shock front. This instability, contrary to the non-resonant streaming instability, is able to generate large scale perturbations which helps to confine high energy CRs.
} 

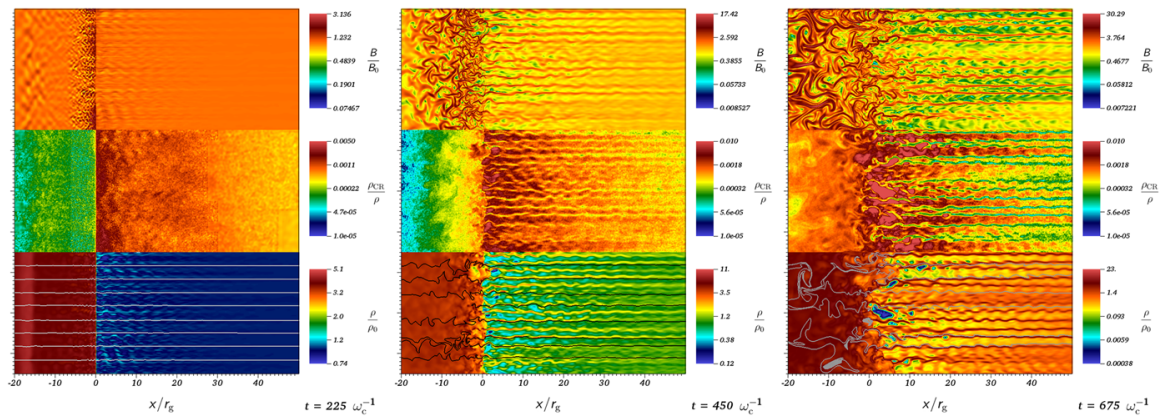

Fig. 41 Super-Alfvénic parallel shock at time $225 \omega_{\mathrm{c}}^{-1}, 450 \omega_{\mathrm{c}}^{-1}$ and $600 \omega_{\mathrm{c}}^{-1}$. The Alfvénic Mach number is $M_{\mathrm{A}}=300$. The upper row shows the magnetic field strength relative to the original background magnetic field. The middle row shows the non-thermal particle charge density relative to the thermal gas density. The lower row shows the thermal gas mass density relative to the upstream density at the start of the simulation, combined with the magnetic field stream lines. Filamentary structures characteristic of the non-resonant streaming instability develop in the upstream medium. The shock front gets strongly corrugated with time. Image reproduced with permission from van Marle et al. (2018), copyright by the authors
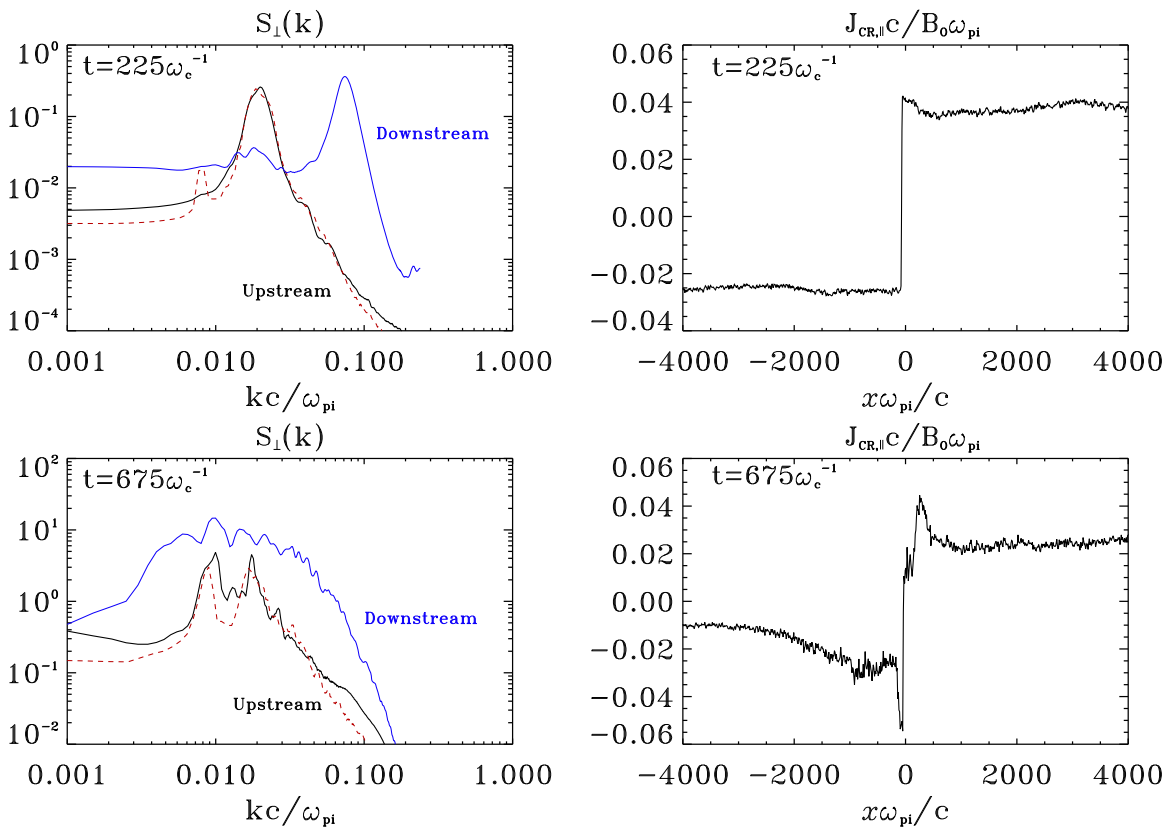

Fig. 42 Fourier spectra of the turbulence in the up- and downstream media at times $225 \omega_{\mathrm{c}}^{-1}$ and $600 \omega_{\mathrm{c}}^{-1}$. At early times the non-resonant Bell-like mode develops at the maximum wavelength predicted by the linear theory. The turbulent scale is smaller in the downstream medium due to to shock compression of the transverse magnetic field component. At later times non-linear effects start to inject turbulent motions at larger scales and modify the upstream CR current. Image reproduced with permission from van Marle et al. (2018), copyright by the authors 
ions are reflected at the shock overshoot, a structure which can not be captured by a MHD code. The main argument in favour is that hybrid simulations have a small simulation box and cannot be performed over duration much exceeding a few hundreds of $c / \omega_{\mathrm{c}, \mathrm{i}}$ so they are not able to capture instabilities that develop at larger scales in the downstream medium once the non-resonant streaming instability is triggered. Moreover, hybrid simulations treat the entire ion population (both thermal and suprathermal) as kinetic and hence have much lower statistics to reconstruct the CR current at the origin of the streaming instability. Both techniques however agree at early timescales for high Alfvénic Mach number shocks and both show the development of a supra-thermal tail associated with the SDA mechanism.

\subsubsection{Other astrophysical applications}

We discuss here recent setups designed to investigate CR or energetic particles transport in specific astrophysical contexts. Two subjects are considered: other type of CR driven instabilities and energetic particles acceleration near $\mathrm{X}$ points in relativistic magnetic reconnection. The number of scientific cases will likely rapidly increase with the availability of numerical resources.

CR driven instabilities In Lucek and Bell (2000) perturbations are produced by the drifting of a mono-energetic or Gaussian distribution of CRs in a background medium at rest. The background magnetic field is composed of a uniform component of strength $\mathrm{B}$ and Alfvén waves of amplitude $\delta B=0.1 B$. CR drift with a speed ten times the local Alfvén speed. The authors consider the growth of the resonant streaming instability and the energy/impulsion transfer to the background plasma using 1-, 2- and 3-D simulations. Rapid magnetic field generation is obtained. The linear growth rate can be sustained even in the non-linear regime. Magnetic field generation saturates when the level of perturbations are $\sim B$.

Lebiga et al. (2018) use a similar numerical approach as the one developed in Bai et al. (2015) (also using the MHD code ATHENA) to investigate the growth of the gyroresonant instability produced by an anisotropic pressure of the CR gas with respect to the background magnetic field. If the strength of the magnetic field changes over scales larger than particle Larmor radius then the first adiabatic invariant $p_{\perp}^{2} / B$ is conserved along the particle trajectory. In this instability the driving term is given by the relative perpendicular to parallel CR pressure $A=P_{\perp} / P_{\|}-1$. The simulation setup involves a background uniform magnetic field on which is superimposed a flat spectrum of circularly polarized MHD waves, and an anisotropic CR energy distribution scaling as $E^{-2.8}$ consistent with the Galactic CR spectrum. The main parameters in the simulations are the ratio of $\mathrm{CR}$ density to gas density $n_{\mathrm{CR}} / n_{\mathrm{g}} \ll 1$, and the initial anisotropy parameter $|A|<1$. The main findings are: the linear growth phase well reproduces the quasi-linear growth rate deduced from Bykov et al. (2013), the non-linear saturation of the instability occurs due to particle isotropization, a process faster at low CR energies which contain most of the CR pressure. Bai et al. (2019) perform PIC-MHD simulations to study the resonant streaming instability. The authors use the $\delta f$ method (see Kunz et al. 2014 and references therein) which consists in affecting a weight $w_{\mathrm{i}}$ to each particle i as: $w_{\mathrm{i}}=1-f\left(\mathbf{x}_{\mathrm{i}}(t), \mathbf{p}_{\mathrm{i}}(t)\right) / f$. This method permits a drastic reduction 
Table 5 A list of the main fluid codes used in astrophysics

\begin{tabular}{lllll}
\hline Code name & physics modules & AMR & geometry & parallelization \\
\hline ATHENA & CR, PIC-MHD & y & $\mathrm{Ca}, \mathrm{Cy}, \mathrm{Sp}, \mathrm{Po}$ & OpenMP/MPI \\
BATS-R-US & PIC-MHD & $\mathrm{y}$ & $\mathrm{Ca}$ & OpenMP/MPI \\
ENZO & $\mathrm{CR}$ & $\mathrm{y}$ & $\mathrm{Ca}, \mathrm{Sp}$ & $\mathrm{MPI}$ \\
MPI-AMRVAC & $\mathrm{CR}$, PIC-MHD, PIC-RMHD & $\mathrm{y}$ & $\mathrm{Ca}, \mathrm{Cy}, \mathrm{Sp}, \mathrm{Po}$ & OpenMP/MPI \\
PIERNIK & $\mathrm{CR}$ & $\mathrm{y}$ & $\mathrm{Cy}$ & $\mathrm{MPI}$ \\
PLUTO & $\mathrm{CR}$, PIC-MHD & $\mathrm{y}$ & $\mathrm{Ca}, \mathrm{Cy}, \mathrm{Sp}, \mathrm{Po}$ & OpenMP/MPI \\
RAMSES & $\mathrm{CR}$ & $\mathrm{y}$ & $\mathrm{Ca}$ & OpenMP/MPI \\
\hline
\end{tabular}

The table displays: the code name, the CR treatment module CR = fluid or PIC-MHD, if or not a relativistic option exists $(\mathrm{SR}=$ special relativistic), if or not an AMR version does exist, the geometrical options $(\mathrm{Ca}=$ Cartesian, $\mathrm{Cy}=$ Cylindrical, $\mathrm{Sp}=$ Spherical, $\mathrm{Po}=$ Polar coordinates $)$, the parallelization model. Code presentation can be found in Stone et al. (2008) for ATHENA, Powell et al. (1999) for BAT-R-US, Bryan et al. (2014) for EnZO, Keppens et al. (2012) for MPI-AMRVAC, Hanasz et al. (2010), for PIERNIK, Mignone et al. (2007) for PLUTO, Teyssier (2002) for RAMSES

of the noise inherently associated with particle simulations. In their fiducial setup the authors inject 2048 particles per cell. The results show that the quasi-linear theory of wave growth is well reproduced for both right- and left-handed mode polarizations. The technique permits to investigate the problem of $90^{\circ}$ pitch-angle scattering which involves non-linear wave particle interactions.

CR acceleration near an X-point Mignone et al. (2018) propose a study of particle acceleration near a 2D X-point in relativistic flows. The simulations are performed in the test-particle limit. The out-of plane electric $E_{\mathrm{Z}}$ and guiding magnetic field $B_{\mathrm{Z}}$ strength are varied. Initially particles are distributed over a Maxwellian with a thermal speed of $0.1 U_{\mathrm{A}}$. Particles are accelerated near the null point where the electric field intensity is the highest. An energy distribution with a power-law approximately $E^{-2}$ is obtained. The particle energy shifts to high energies as the guiding magnetic field strength increases because the acceleration zone where $\mathbf{E} . \mathbf{B} \neq 0$ extends with respect to the case when $B_{\mathrm{Z}}$ is smaller than the in-plane magnetic field strength.

\subsection{A list of HD and MHD codes with CR physics}

In Table 5, we present the MHD codes in use in various astrophysical applications that include a module (fluid or kinetic) to treat CR physics, a more complete list can be found in the appendix of Martí and Müller (2015).

\subsection{Semi-analytical approaches for cosmic ray acceleration}

Because direct simulations of DSA are numerically expensive, simpler approaches are desirable for the modelling of large-scale and multi-physics problems. Most of the methods described above deal with the microphysics of collisionless shocks and particle acceleration in some way, and are not readily applicable to simulate a 
macroscopic object like, say, a supernova remnant. The most precise methods, PIC simulations, are restricted to tiny scales and narrow dynamical ranges. For instance, in the most advanced numerical simulations of DSA relevant to SNRs to date, by Caprioli and Spitkovsky (2014b), lengths are normalized by $l_{p}=c / \omega_{p}$ where $\omega_{p}$ is the ion plasma frequency, which for a typical density $n_{p}=1 \mathrm{~cm}^{-3}$ evaluates to $l_{p} \simeq 2 \times 10^{7} \mathrm{~cm}=2 \times 10^{-12}$ pc. So in their Figs. 6 and 7 , the "far upstream" reaches about $3 \times 10^{-7} \mathrm{pc}$, whereas in state of the art simulations of the $3 \mathrm{D}$ evolution of a young SNR, by Ferrand et al. (2010), the smallest length resolved is $\simeq 5 \mathrm{pc} / 1024 \simeq 5 \times 10^{-3} \mathrm{pc}$, nearly 5 orders of magnitude larger. Similarly in the same figures the range of magnetic turbulence scales probed is $\sim 10^{-11}-10^{-8} \mathrm{pc}$, whereas in one of the most recent study of the effect of turbulence on the SNR emission, by West et al. (2017), the smallest resolved scale is $4 \times 10^{-2} \mathrm{pc}, 6$ to 9 orders of magnitude larger. It is therefore necessary to make use of sub-grid models in simulations of astrophysical objects. In hydro-kinetic approaches of Sect. 5.4.1, the complex interaction between the particles and the magnetic turbulence is encapsulated in the diffusion coefficient $D$. With such approaches it is possible to operate on space- and time-scales that are relevant to an object like a SNR (e.g. Kang 2015), although simulations still have a high computational cost when using a realistic dependence of $D$ on $p$, and to our knowledge their use has been restricted to 1-dimensional problems (in either slab or spherically symmetric geometries). When the focus is on properly describing the geometry of the SNR, and a 3-dimensional modeling is required, the treatment of DSA needs to be simplified even further.

\subsubsection{General considerations}

To fill this need, Berezhko and Ellison (1999) proposed a simple analytical model of NLDSA, where the spectrum of the particles is assumed to be a three-parts powerlaw, with slopes linked to the shock properties. This model was used to make the first studies of the effect of NLDSA on young SNRs, by coupling it to 1D selfsimilar solutions (Decourchelle et al. 2000) then to 1D hydrodynamic simulations (Ellison et al. 2004). A more physical, semi-analytical model was proposed by Blasi (2002), in the framework of the hydro-kinetic treatment. The key idea (developed in the next section), which allows to greatly simplify the mathematics, is that the energydependent diffusion of the particles allows to establish a one-to-one correspondence between the particle energy $E$ (or equivalently momentum $p$ ), the position variable $x$, and the fluid velocity $u$. This trick was already used in Eichler (1979) and developed by Eichler (1984), Ellison and Eichler (1984), Berezhko (1996), but somehow its usefulness was not fully realized until Blasi $(2004 ; 2005)$ published their NLDSA model.

Being physically motivated while computationally extremely fast, Blasi's model was quickly adopted by the community doing SNR simulations: it replaced the Berezhko and Ellison model in the 1D hydrodynamic simulations of Ellison et al. (2007) and following works, and it was used for the 3D hydrodynamical simulations of Ferrand et al. (2010) and following works. It allowed Kosenko et al. (2014) to perform parametric studies of the efficiency of CR acceleration in young SNRs. Note that an important limitation of the model is that it is looking only for stationary solutions, 
and so needs to be re-run at each time step in order to compute the time evolution of the coupled shock-particles system, assuming quasi-stationarity is reached at each step. This is justified at most energies, but will break down close to the highest energies when the acceleration time becomes of the same order as the age of the simulated shock. The model jointly solves the particle spectrum and the fluid velocity profile as functions of the momentum of particles. As inputs, it requires basic information on the shock (speed $V_{\mathrm{sh}}$ and Mach number $M_{\mathrm{sh}}$ ), which can be determined from a hydrodynamic simulation, as well as an injection recipe at some $p_{\text {inj }}$ and a cutoff recipe to set $p_{\max }$. Amongst the outputs, it provides the total shock compression ratio $r_{\text {tot }}$, that can be used to determine an effective adiabatic index $\gamma_{\text {eff }}$ for the fluid+particles system. The back-reaction of the particles on the flow can then be imposed by tweaking the value of $\gamma$ in the hydro model according to the prediction of the NLDSA model. Ellison et al. (2004) showed good agreement between this pseudo-fluid approach and two-fluid calculations in 1D. It is worth mentioning that in the original (and most popular) version of Blasi's model one does not deal explicitly with the diffusion coefficient $D$ (although often an assumption on $D(p)$ is made in order to estimate $p_{\max }(t)$ ). Accordingly, hydrodynamic simulations typically do not need to explicitly resolve the shock precursor generated by the particles, although for a given $D(p)$ law the velocity profile in the precursor may be reconstructed if desired (the position $x_{p}$ where the fluid velocity is $u_{p}$ is given by $\left.x_{p}=D(p) / u_{p}\right)$. In a subsequent model, Amato and Blasi (2005) introduced the explicit spatial dependence of the distribution $f$ and the diffusion coefficient $D$. This generalization is more complex to derive and significantly longer to compute, and Amato et al. (2008) showed that the two models provide similar results.

The simple model was gradually improved to incorporate other physical processes. Of particular importance is the improvement of the treatment of the magnetic turbulence (Caprioli et al. 2008, 2009b). A recipe for magnetic field amplification (MFA) was included, and the fate of magnetic waves generated by the particles was considered: they may either be damped in the plasma upstream of the shock or be carried through the shock, which leads to very different magnetic fields in the downstream region, although similar overall levels of back-reaction on the shock. These effects were included in SNR simulations as well (by Lee et al. 2012 in 1D and Ferrand et al. 2014b in 3D). Another point of interest, regarding SNRs as sources of cosmic rays, is the role of particles escaping the accelerator. In the base model only the spectrum at the shock is computed and escape is treated implicitly, but if desired the escape of particles can be treated explicitly, in two ways (Caprioli et al. 2009a, 2010a). Other recent developments include the role of ionization (Morlino 2011) and the role of neutrals (Morlino et al. 2013) in the DSA process.

A comparison between Blasi's semi-analytical model and two popular numerical approaches, hydro-kinetic simulations à la Kang \& Jones (Sect. 5.4.1) and Monte Carlo simulations à la Ellison \& Eichler (Sect. 3.6.3), can be found in Caprioli et al. (2010b). Typical results are shown in Fig. 43.

In the following last section, we outline the inner workings of Blasi's NLDSA model. 

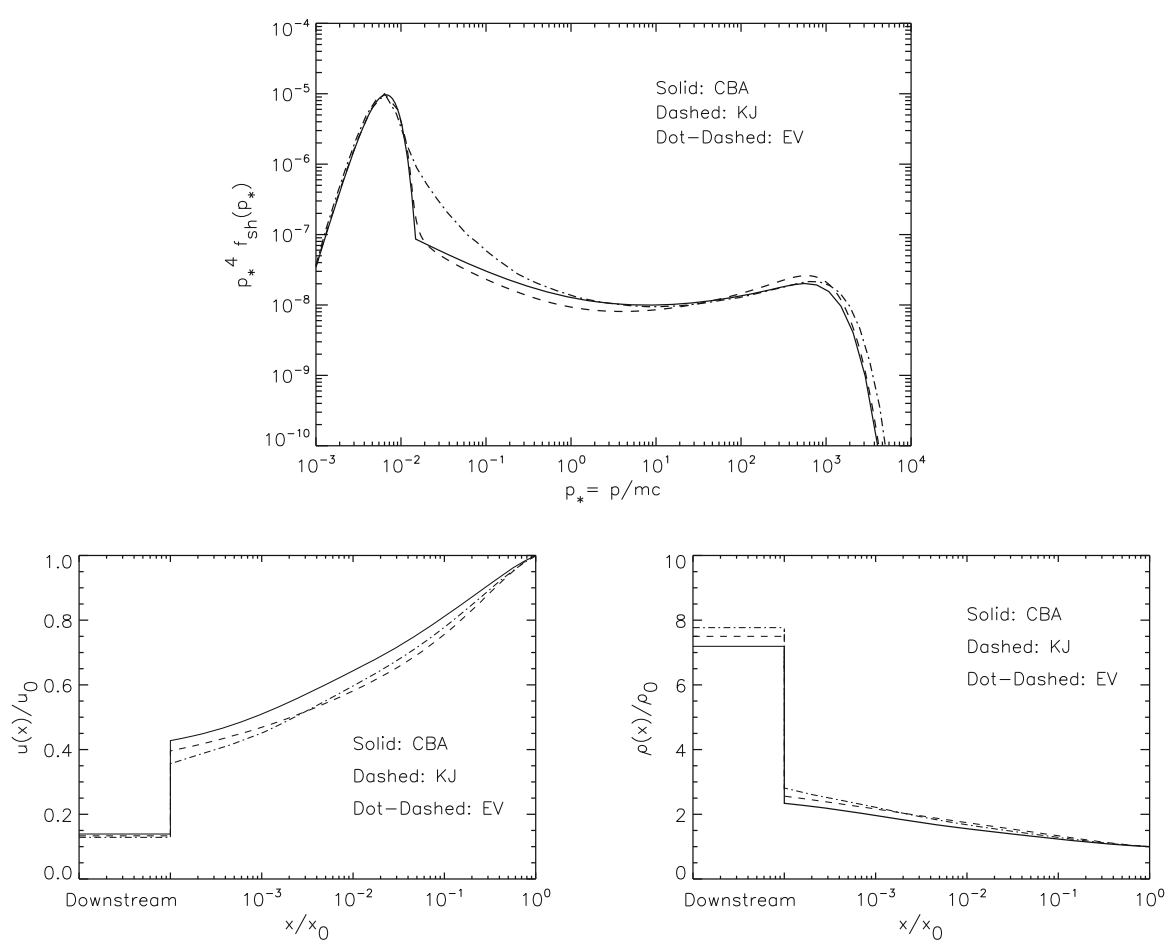

Fig. 43 Comparison of different methods for non-linear diffusive shock acceleration. The plot at the top shows the distribution function $f(p)$ of particles, the Maxwellian component and a non-thermal tail are apparent. The two plots at the bottom show the hydro profiles of the shock: velocity $u(x)$ and density $\rho(x)$. The models assume efficient acceleration and include backreaction effects: the reduced sub-shock and extended precursor are apparent, and accordingly the non-thermal spectrum is concave. The three different sets of curves show the results of three different methods to solve the coupled fluid and particles system: a semi-analytical model (solid lines, labelled CBA for Caprioli-Blasi-Amato, described in Sect. 5.7.2), a numerical model coupling hydro equations and a diffusion-convection equation (dashed lines, labelled KJ for Kang and Jones, described in Sect. 5.4.1), and another numerical model relying on a Monte Carlo approach (dot-dashed lines, labelled EV for Ellison \& Vladimirov, described in Sect. 3.6.3). Images reproduced with permission from Caprioli et al. 2010b, copright by the authors

\subsubsection{Construction of a NLDSA model}

We restrict ourselves to 1D slab geometry along direction $x$, with a velocity discontinuity (sub-shock) located at $x=0$, and a velocity ramp (precursor) extending for $x<0$ over a distance $x_{\max }$. Subscripts distinguish between three distinct media, with usual notations: 0 denotes the far upstream (unperturbed) medium $\left(x<-x_{\max }\right)$, 1 denotes the region immediately upstream of the sub-shock $\left(x=0^{-}\right)$, and 2 the region downstream of the sub-shock $\left(x=0^{+}\right)$.

Distribution of accelerated particles at the shock The evolution of the distribution function $f$ is described by a convection-diffusion equation which, under the assumption of stationarity $\partial f / \partial t=0$ reads 


$$
-u \frac{\partial f}{\partial x}+\frac{\partial}{\partial x}\left(D \frac{\partial f}{\partial x}\right)+\frac{1}{3} \frac{\mathrm{d} u}{\mathrm{~d} x} p \frac{\partial f}{\partial p}+Q=0
$$

where $D$ is the diffusion coefficient, and $Q$ represents injection of particles, assumed to occur only at the shock front: $Q(x, p)=Q_{1}(p) \delta(x)$. By integrating Eq. (108) across the shock (from $x=0^{-}$to $x=0^{+}$), using the continuity of the distribution function $f_{2}=f_{1}$, then integrating it from far upstream $\left(x<-x_{\max }\right)$ to just ahead of the sub-shock $\left(x=0^{-}\right)$, and assuming homogeneity downstream and of the shock: $(\partial f / \partial x)_{2}=0$, the equation can be recast as

$$
\frac{1}{3}\left(u_{p}-u_{2}\right) p \frac{\mathrm{d} f_{1}}{\mathrm{~d} p}-\left(u_{p}+\frac{1}{3} p \frac{\mathrm{d} u_{p}}{\mathrm{~d} p}\right) f_{1}+\left(u_{0} f_{0}+Q_{1}\right)=0,
$$

where we have introduced the key function

$$
u_{p}=u_{1}-\frac{1}{f_{1}} \int_{-x_{\max }}^{0^{-}} \mathrm{d} x \frac{\mathrm{d} u}{\mathrm{~d} x} f(x, p)
$$

which is the average fluid velocity experienced by particles with momentum $p$ while diffusing upstream of the shock front. Assuming that $D$ is a growing function of $p$, particles of a given momentum $p$ explore a region of a certain extent $x_{p}$ upstream of the shock, and thus sample only a part of the precursor in the velocity profile. Hence, $u_{p}$ can be thought of as being the typical velocity of the fluid at the point $x_{p}$ that particles of momentum $p$ can reach.

The solution of Eq. (109) can be written in implicit form

$$
f_{1}(p)=\frac{3}{U_{p}-R_{\mathrm{tot}}^{-1}} \int_{p_{\min }}^{p} \frac{\mathrm{d} p^{\prime}}{p^{\prime}}\left(f_{0}\left(p^{\prime}\right)+\frac{Q_{1}\left(p^{\prime}\right)}{u_{0}}\right) \exp \left(-\int_{p^{\prime}}^{p} \frac{\mathrm{d} p^{\prime \prime}}{p^{\prime \prime}} \frac{3 U_{p^{\prime \prime}}}{U_{p^{\prime \prime}}-R_{\mathrm{tot}}^{-1}}\right)
$$

where we have noted $p_{\min }$ the minimum momentum of particles and we have introduced the total compression of the shock $R_{\text {tot }}=u_{0} / u_{2}$ and, in a similar way, the normalized velocity everywhere in the precursor: $U_{p}=u_{p} / u_{0}$.

If we further assume that there are no pre-existing particles: $f_{0}=0$, and that a fraction $\eta$ of the particles crossing the sub-shock are "injected" in the acceleration process at a single momentum $p_{\text {inj }}$ :

$$
Q_{1}(p)=\frac{\eta n_{1} u_{1}}{4 \pi p_{\text {inj }}^{2}} \delta\left(p-p_{\text {inj }}\right)
$$

then Eq. (111) simplifies to

$$
f_{1}(p)=\frac{3}{U_{p}-R_{\mathrm{tot}}^{-1}} \frac{\eta n_{0}}{4 \pi p_{\mathrm{inj}}^{3}} \exp \left(-\int_{p_{\mathrm{inj}}}^{p} \frac{\mathrm{d} p^{\prime}}{p^{\prime}} \frac{3 U_{p^{\prime}}}{U_{p^{\prime}}-R_{\mathrm{tot}}^{-1}}\right)
$$


The injection momentum $p_{\text {inj }}$ can be parametrized as

$$
p_{\text {inj }}=\xi p_{\text {th }, 2}
$$

where $p_{\text {th,2 }}=\sqrt{2 m_{p} k T_{2}}$ is the mean downstream thermal momentum. Continuity of the thermal and non-thermal distributions at $p_{\text {inj }}$ imposes that

$$
\eta=\frac{4}{3 \sqrt{\pi}}\left(R_{\mathrm{sub}}-1\right) \xi^{3} \exp \left(-\xi^{2}\right),
$$

where we have introduced the compression of the sub-shock $R_{\text {sub }}=u_{1} / u_{2}$. The factor $R_{\text {sub }}-1$ acts as a regulator: injection is switched off when the sub-shock gets smoothed. We have a single parameter $\xi$ to describe injection, but note that the value of $\eta$ is extremely sensitive to the value of $\xi$.

Velocity profile of the fluid in the precursor In the previous paragraph we have expressed the distribution of accelerated particles $f$ as a function of the velocity profile of the thermal fluid $U$. As particles back-react on the shock dynamics, $U$ is itself a function of $f$. To find this second relation, we make use of conservation of momentum, which involves 4 terms: dynamical pressure $\rho u^{2}$, thermal pressure $P_{\text {th }}$, non-thermal pressure $P_{\mathrm{CR}}$, and waves pressure $P_{\mathrm{w}}$. We write it from a point far upstream $\left(x<-x_{\max }\right)$, where the fluid velocity is $u_{0}$, to the point $x_{p}$ (reached by particles of momentum $p$ ), where the fluid velocity is $u_{p}$ :

$$
\rho_{p} u_{p}^{2}+P_{\mathrm{th}, p}+P_{\mathrm{cr}, p}+P_{\mathrm{w}, p}=\rho_{0} u_{0}^{2}+P_{\mathrm{th}, 0}+P_{\mathrm{cr}, 0}+P_{\mathrm{w}, 0} .
$$

The upstream fluid pressure $P_{\mathrm{th}, 0}$ can be expressed as

$$
P_{\mathrm{th}, 0}=\frac{\rho_{0} u_{0}^{2}}{\gamma_{\mathrm{th}} M_{S, 0}^{2}}
$$

Assuming adiabatic compression, the fluid pressure $P_{\mathrm{th}, p}$ at any point $x_{p}$ in the precursor is given by

$$
\frac{P_{\mathrm{th}, p}}{P_{\mathrm{th}, 0}}=\left(\frac{\rho_{p}}{\rho_{0}}\right)^{\gamma_{\mathrm{th}}}=U_{p}^{-\gamma_{\mathrm{th}}}
$$

where in the second equality we have made use of the conservation of mass. Various processes may lead to non-adiabatic compression in the precursor. One of the most discussed is heating through the damping of Alfvén waves, for which Berezhko and Ellison (1999) propose the following recipe (obtained for large $M_{A, 0}$ ):

$$
\frac{P_{\mathrm{th}, p}}{P_{\mathrm{th}, 0}}=U_{p}^{-\gamma_{\mathrm{th}}}\left(1+\zeta\left(\gamma_{\mathrm{th}}-1\right) \frac{M_{S, 0}^{2}}{M_{A, 0}}\left(1-U_{p}^{\gamma_{\mathrm{th}}}\right)\right)
$$

where $M_{A, 0}$ is the upstream Alfvénic Mach number, and $\zeta \in[0,1]$ is a free parameter added by Caprioli et al. (2009b) and discussed more below. 
The particle pressure $P_{\mathrm{cr}, p}=P_{\mathrm{cr}, p, 0}+P_{\mathrm{cr}, p, 1}$ at point $x_{p}$ is the sum of two terms. The first term is the pressure contributed by the adiabatic compression of an upstream population $f_{0}$ :

$$
P_{\mathrm{cr}, p, 0}=U_{p}^{-\gamma_{\mathrm{cr}}} \times P_{\mathrm{cr}, 0}
$$

where $\gamma_{\mathrm{cr}} \simeq 4 / 3$ is the adiabatic index of the particles "fluid" and

$$
P_{\mathrm{cr}, 0}=\int_{p_{\min , 0}}^{p_{\max , 0}} \frac{p^{\prime} v\left(p^{\prime}\right)}{3} f_{0}\left(p^{\prime}\right) 4 \beta \mathrm{p}^{\prime 2} \mathrm{~d} p^{\prime}=\frac{4 \pi}{3} m_{p} c^{2} \int_{p_{\min , 0}}^{p_{\max , 0}} \frac{p^{\prime 4} f_{0}\left(p^{\prime}\right)}{\sqrt{1+p^{\prime 2}}} \mathrm{~d} p^{\prime}
$$

(with momenta expressed in $m_{p} c^{2}$ units in the right expression). The second term is is the pressure of the particles accelerated at the shock (with distribution $f_{1}(p)$ extending up to $p_{\max , 1}$ ) and able to reach the position $x_{p}$ (that is those of momenta $\geq p)$ :

$$
P_{\mathrm{cr}, p, 1}=\int_{p}^{p_{\max , 1}} \frac{p^{\prime} v\left(p^{\prime}\right)}{3} f_{1}\left(p^{\prime}\right) 4 \beta \mathrm{p}^{\prime 2} \mathrm{~d} p^{\prime}=\frac{4 \pi}{3} m_{p} c^{2} \int_{p}^{p_{\max , 1}} \frac{p^{\prime 4} f_{1}\left(p^{\prime}\right)}{\sqrt{1+p^{\prime 2}}} \mathrm{~d} p^{\prime} .
$$

Finally we turn to the pressure in magnetic waves. Far upstream, we assume that the magnetic field is not turbulent: $P_{\mathrm{w}, 0}=0$. In the precursor, particles are believed to generate themselves the turbulence required for their scattering, hence as a first approach we may parametrize the pressure of waves as being some fraction $\alpha<1$ of the pressure of particles:

$$
P_{\mathrm{w}, p}=\alpha P_{\mathrm{cr}, p} .
$$

According to quasi-linear theory, $\alpha \sim v_{A} / u_{0}$ for the resonant streaming instability, and $\alpha \sim u_{0} / c$ for the non-resonant modes. Caprioli et al. (2009b) propose the following recipe for the resonant instability (obtained for large $M_{S, 0}$ and $M_{A, 0}$ ):

$$
\frac{P_{\mathrm{w}, p}}{\rho_{0} u_{0}^{2}}=\frac{1-\zeta}{4 M_{A, 0}} U_{p}^{-3 / 2}\left(1-U_{p}^{2}\right)
$$

The term $U_{p}^{-3 / 2}$ represents adiabatic compression. The factor $1-\zeta$ is introduced to balance the factor $\zeta$ in relation (119): the amount of wave damping has to remain reasonably small for the magnetic field to be substantially amplified. Different parametrizations of $\delta B$ in the precursor are possible, see Kang et al. (2013) for a comparison of four different models.

Using the above relations for $P_{\mathrm{th}}, P_{\mathrm{cr}}, P_{\mathrm{w}}$, together with mass conservation, Eq. (116) can be written as 


$$
\begin{aligned}
& U_{p}+\left(\begin{array}{l}
\frac{U_{p}^{-} \gamma_{\mathrm{th}}}{\gamma_{\mathrm{th}} M_{S, 0}^{2}}\left(1+\zeta\left(\gamma_{\mathrm{th}}-1\right) \frac{M_{S, 0}^{2}}{M_{A, 0}}\left(1-U_{p}^{\gamma_{\mathrm{th}}}\right)\right) \\
+\frac{U_{p}^{-} \gamma_{\mathrm{cr}, 0}}{\gamma_{\mathrm{th}} M_{S, 0}^{2}} \frac{P_{\mathrm{cr}, 0}}{P_{\mathrm{th}, 0}}+\frac{4 \pi}{3} \frac{m_{p} c^{2}}{\rho_{0} u_{0}^{2}} \int_{p}^{p_{\mathrm{max}, 1}} \frac{p^{\prime 4} f_{1}\left(p^{\prime}\right)}{\sqrt{1+p^{\prime 2}}} \mathrm{~d} p^{\prime} \\
+\frac{1-\zeta}{4 M_{A, 0}} \frac{1-U_{p}^{2}}{U_{p}^{3 / 2}}
\end{array}\right) \\
& =1+\frac{1}{\gamma_{\mathrm{th}} M_{S, 0}^{2}}\left(1+\frac{P_{\mathrm{cr}, 0}}{P_{\mathrm{th}, 0}}\right) .
\end{aligned}
$$

Deriving relation (125) with respect to $p$, we finally obtain

$$
\begin{aligned}
& \left(1-\frac{U_{p}^{-\left(\gamma_{\mathrm{th}}+1\right)}}{M_{S, 0}^{2}}\left(1+\zeta\left(\gamma_{\mathrm{th}}-1\right) \frac{M_{S, 0}^{2}}{M_{A, 0}}+\frac{\gamma_{\mathrm{cr}, 0}}{\gamma_{\mathrm{th}}} \frac{P_{\mathrm{cr}, 0}}{P_{\mathrm{th}, 0}} \frac{U_{p}^{-\gamma_{\mathrm{cr}, 0}}}{U_{p}^{-\gamma_{\mathrm{th}}}}\right)-\frac{1-\zeta}{8 M_{A, 0}} \frac{U_{p}^{2}+3}{U_{p}^{5 / 2}}\right) \\
& \times \frac{\mathrm{d} U_{p}}{\mathrm{~d} p}=\frac{4 \pi}{3} \frac{m_{p} c^{2}}{\rho_{0} u_{0}^{2}} \frac{p^{4} f_{1}(p)}{\sqrt{1+p^{2}}} .
\end{aligned}
$$

The distribution of particles $f_{1}$ at the shock being known, the velocity profile $U_{p}$ of the fluid can be computed by integrating Eq. (126) from one of these two boundary conditions to the other:

$$
\begin{gathered}
U_{p}(p=0)=U_{p}\left(x=0^{-}\right)=\frac{1}{R_{\text {prec }}}, \\
U_{p}\left(p=p_{\max }\right)=U_{p}\left(x=-x_{\max }\right)=1,
\end{gathered}
$$

where we have introduced the compression factor of the whole precursor $R_{\text {prec }}=$ $u_{0} / u_{1}$. In practice, we will be looking for a $R_{\text {prec }}$ such that, starting from condition (127), condition (128) is matched after integration of Eq. (126).

Compression at the sub-shock So far we have expressed $f_{1}$ as a function of $U_{p}, R_{\text {tot }}$ and $R_{\text {sub }}$ [Eq. (111) with injection recipe (112)-(115)], and $U_{p}$ as a function of $f_{1}$ and $R_{\text {prec }}$ [Eq. (126) with boundary conditions (127)-(128)], To solve the coupled system $f_{1}-U_{p}$, we need another independent relation between any two of the three compression ratios $R_{\text {prec }}, R_{\text {sub }}$ and $R_{\text {tot }}$ (the third one being deduced from $R_{\text {tot }}=$ $R_{\text {prec }} \times R_{\text {sub }}$ ). To obtain this relation, we once again use conservation of momentum, this time across the sub-shock (from $x=0^{-}$to $x=0^{+}$):

$$
\rho_{2} u_{2}^{2}+P_{\mathrm{th}, 2}+P_{\mathrm{cr}, 2}+P_{\mathrm{w}, 2}=\rho_{1} u_{1}^{2}+P_{\mathrm{th}, 1}+P_{\mathrm{cr}, 1}+P_{\mathrm{w}, 1}
$$

The pressure of accelerated particles is always continuous across the shock: $P_{\mathrm{cr}, 2}=$ $P_{\mathrm{cr}, 1}$. For the magnetic waves, using a simplified treatment of the parallel shock and in the limit of large Alfvénic numbers, Caprioli et al. (2008, 2009b) estimate the jump in pressure $P_{\mathrm{w}}$ and in energy flux $F_{\mathrm{w}}$ to be 


$$
\begin{aligned}
& {\left[P_{\mathrm{w}}\right]_{1}^{2}=R_{\mathrm{sub}}^{2}-1,} \\
& {\left[F_{\mathrm{w}}\right]_{1}^{2}=2\left(R_{\mathrm{sub}}-1\right) P_{\mathrm{w}, 1} u_{1} .}
\end{aligned}
$$

Then the jump in fluid pressure at the shock is

$$
\frac{P_{\mathrm{th}, 2}}{P_{\mathrm{th}, 1}}=\frac{\left(\gamma_{\mathrm{th}}+1\right) R_{\mathrm{sub}}-\left(\gamma_{\mathrm{th}}-1\right)\left(1-\left(R_{\mathrm{sub}}-1\right)^{3} \frac{P_{\mathrm{w}, 1}}{P_{\mathrm{th}, 1}}\right)}{\left(\gamma_{\mathrm{th}}+1\right)-\left(\gamma_{\mathrm{th}}-1\right) R_{\mathrm{sub}}} .
$$

Substituting relations (132) and (130) in Eq. (129), and using mass conservation, we obtain

$$
M_{S, 1}^{2}=\frac{2 R_{\mathrm{sub}}}{\left(\gamma_{\mathrm{th}}+1\right)-\left(\gamma_{\mathrm{th}}-1\right) R_{\mathrm{sub}}-2 R_{\mathrm{sub}}\left(\gamma_{\mathrm{th}}-\left(\gamma_{\mathrm{th}}-2\right) R_{\mathrm{sub}}\right) P_{\mathrm{w}, 1}^{\star}}
$$

where we have introduced the sonic Mach number $M_{S, 1}$ of the sub-shock, and where we have noted

$$
P_{\mathrm{w}, 1}^{\star}=\frac{P_{\mathrm{w}, 1}}{\rho_{1} u_{1}^{2}}=R_{\mathrm{prec}} \frac{P_{\mathrm{w}, 1}}{\rho_{0} u_{0}^{2}}=\frac{1-\zeta}{4 M_{A, 0}} R_{\mathrm{prec}}^{5 / 2}\left(1-R_{\mathrm{prec}}^{-2}\right),
$$

where we used recipe (124) for the last equality. In the case where the pressure of magnetic waves is negligible $\left[P_{\mathrm{w}, 1} \simeq 0\right.$, that is $\zeta \simeq 1$ with recipe (124)], Eq. (133) reduces to the well-known hydrodynamics relation

$$
M_{S, 1}^{2}=\frac{2 R_{\mathrm{sub}}}{\left(\gamma_{\mathrm{th}}+1\right)-\left(\gamma_{\mathrm{th}}-1\right) R_{\mathrm{sub}}} \Longleftrightarrow R_{\mathrm{sub}}=\frac{\left(\gamma_{\mathrm{th}}+1\right) M_{S, 1}^{2}}{\left(\gamma_{\mathrm{th}}-1\right) M_{S, 1}^{2}+2}
$$

In the case $P_{\mathrm{w}, 1}>0$, Eq. (133) is a quadratic relation for $R_{\text {sub }}$ as a function of $M_{s, 1}$ and $P_{\mathrm{w}, 1}^{\star}$, and thus of $R_{\text {prec. }}$. We can thus solve the sub-shock.

Alfvénic drift At this point, we should make the distinction between the velocity of the flow $u$, and the velocity of the scattering centers $\tilde{u}$. In the MFA picture Alfvén waves are generated by particles counter-streaming the flow, so that in the precursor $\tilde{u}_{p}=u_{p}-v_{A, p}$ where $v_{A, p}$ is the Alfvén speed at the location reached by particles of momentum $p$, while in the downstream region $\tilde{u}_{2}=u_{2}+v_{A, 2}$. It is this velocity $\tilde{u}$ that should be used in the transport equation for the particles. The effective velocity jumps experienced by the particles are smaller than $R_{\text {sub }}$ and $R_{\text {tot }}$, which leads to steeper spectra. In the preceding derivation we have assumed $u \simeq \tilde{u}$ for simplicity, but if MFA is efficient this may not be true. Now a difficulty is that, when the magnetic field is strongly turbulent, it is not clear how the waves speed should be calculated. The common approach (used, e.g., by Lee et al. 2012; Kang 2012; Ferrand et al. 2014b) is to parametrize the Alfvénic drift in the form 


$$
v_{A, p}=\frac{B_{0}+f_{A} \times\left(B_{p}-B_{0}\right)}{\sqrt{4 \pi \rho}},
$$

where $B_{p}=\sqrt{B_{0}^{2}+\delta B_{p}^{2}}$ is the total magnetic field at point $x_{p}$ and we have introduced the free parameter $f_{A} \in[0,1]$. In this model, MFA is thus described by two free parameters $\zeta$ [Eqs. (119) and (124)] and $f_{A}$. The Alfvénic drift is an important correction when $\zeta$ is close to 0 and $f_{A}$ is close to 1 .

Escaping flux A steady-state solution to the problem can only exist if particles can escape above some maximum momentum $p_{\max }$, or upstream of some maximum diffusion length $x_{\max }$. In the model, the two approaches are equivalent, the two quantities being related through the relation $x_{\max }=D\left(p_{\max }\right) / u_{0}$. However, the two approaches do not provide the same information on the escape of particles: imposing $f\left(x_{\max }\right)=0$ allows to compute the energy spectrum $\phi_{0}(p)$ of particles leaving the shock around $p_{\max }$, whereas imposing $f\left(p_{\max }\right)=0$ only allows to compute the net (integrated) energy flux $F_{\mathrm{esc}, 0}$ at the boundary $x_{\max }$.

If one integrates Eq. (108) from the point $x_{\max }$ where particles are supposed to leave the system, defined so that $f\left(x_{\max }\right)=0$, then a new term $\phi_{0}$ appears on the 1.h.s. of Eq. (109):

$$
\phi_{0}=-D\left(\frac{\partial f}{\partial x}\right)_{0}
$$

which is the flux of particles leaving the system through the boundary $x_{\max }$. Assuming as before that no seed particles are present upstream and that injection at the shock front is mono-energetic, the solution (113) becomes

$$
f_{1}(p)=\frac{3}{U_{p}-R_{\mathrm{tot}}^{-1}} \frac{\eta n_{0}}{4 \pi p_{\mathrm{inj}}^{3}} \exp \left(-\int_{p_{\mathrm{inj}}}^{p} \frac{\mathrm{d} p^{\prime}}{p^{\prime}} \frac{3\left(U_{p^{\prime}}+\Phi_{0}\left(p^{\prime}\right)\right)}{U_{p^{\prime}}-R_{\mathrm{tot}}^{-1}}\right)
$$

where we have noted the normalized escape flux $\Phi_{0}(p)=\phi_{0}(p) /\left(u_{0} f_{1}(p)\right)$. According to Caprioli et al. (2010a), to a very good approximation we have

$$
\frac{1}{\Phi_{0}(p)}=\int_{0}^{x_{\max }} \mathrm{d} x^{\prime} \frac{u_{0}}{D\left(x^{\prime}, p\right)} \exp \left(-\int_{0}^{x^{\prime}} \mathrm{d} x " \frac{u\left(x^{\prime \prime}\right)}{D\left(x^{\prime}, p\right)}\right) .
$$

The net flux of energy through the upstream boundary is

$$
\begin{aligned}
F_{\mathrm{esc}, 0} & =\int_{p_{\mathrm{inj}}}^{p_{\max }} K\left(p^{\prime}\right) \phi_{0}\left(p^{\prime}\right) 4 \pi p^{\prime 2} \mathrm{~d} p^{\prime} \\
& =4 \pi m_{p} c^{2} \int_{p_{\min }}^{p_{\max }}\left(\sqrt{1+p^{\prime 2}}-1\right) p^{\prime 2} \phi_{0}\left(p^{\prime}\right) \mathrm{d} p^{\prime},
\end{aligned}
$$

where $K(p)$ is the kinetic energy of a particle of momentum $p$ (expressed in $m_{p} c^{2}$ units in the right expression). 
If one integrates Eq. (108) from sufficiently far upstream, one can assume that the upstream gradient of particles vanishes, so that $\phi_{0}=0$. One can still compute the flux of escaping energy, by requesting that the particle distribution vanishes at the maximum momentum $p_{\max }: f\left(p_{\max }\right)=0$. From Caprioli et al. (2009a), this condition imposes that

$$
F_{\mathrm{esc}, 0}=\frac{4 \pi}{3}\left(u_{2}-u_{0}\right) p_{\max }^{3} K\left(p_{\max }\right) f\left(p_{\max }\right),
$$

where $K(p)$ is the kinetic energy of a particle of momentum $p$.

Finally we note that, to obtain all the required relations between hydrodynamic and kinetic quantities, we have only made use of the conservation of mass and of the conservation of momentum. Once the particle distribution, shock velocity profile, and escape flux have been obtained, the third conservation law, namely conservation of energy, can be checked, which provides a way to assess the precision of the model. We write it between upstream $\left(x=-x_{\max }\right)$ and downstream of the shock $\left(x=0^{+}\right)$:

$$
\begin{aligned}
& \frac{1}{2} \rho_{2} u_{2}^{3}+\frac{\gamma_{\text {th }}}{\gamma_{\text {th }}-1} P_{\text {th }, 2} u_{2}+\frac{\gamma_{\mathrm{cr}}}{\gamma_{\mathrm{cr}}-1} P_{\mathrm{cr}, 2} u_{2}+F_{\mathrm{w}, 2} \\
& \quad=\frac{1}{2} \rho_{0} u_{0}^{3}+\frac{\gamma_{\mathrm{th}}}{\gamma_{\mathrm{th}}-1} P_{\mathrm{th}, 0} u_{0}+\frac{\gamma_{\mathrm{cr}}}{\gamma_{\mathrm{cr}}-1} P_{\mathrm{cr}, 0} u_{0}+F_{\mathrm{w}, 0}-F_{\mathrm{esc}, 0},
\end{aligned}
$$

where the different terms account for kinetic energy, thermal energy, CR pressure, magnetic waves pressure, and CR escape.

Procedure for solving the coupled problem As a summary, we outline the practical way for the numerical resolution of the system. For a given compression $R_{\text {prec }}$ in the precursor, the non-linearly coupled system $(f, U)$ can be solved iteratively as follows:

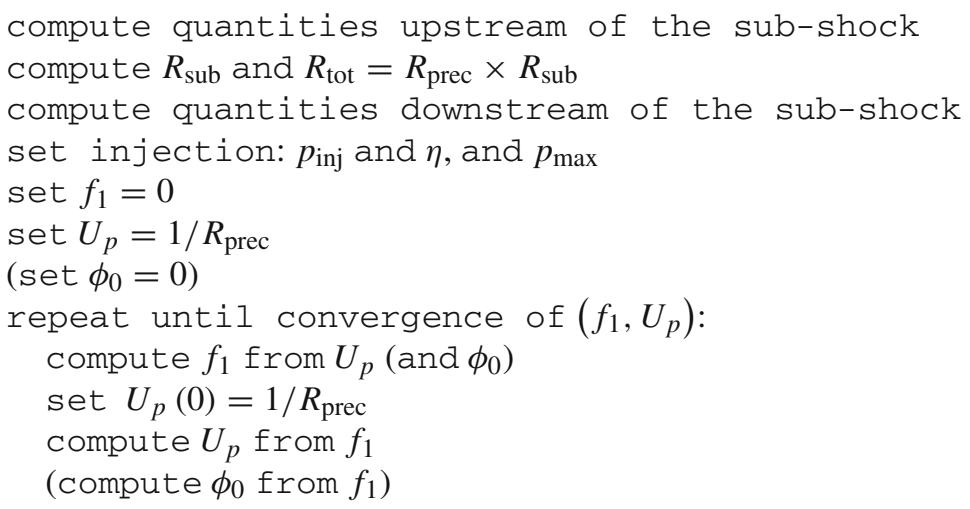

We have neglected the Alfvénic drift for simplicity, and the lines in parentheses apply only when computing particle escape by imposing a spatial boundary condition. Possible values for $R_{\text {prec }}$ range from 1 (no precursor, the shock is not modified, $M_{S, 1}=$ $M_{S, 0}$ and $\left.R_{\text {sub }}=R_{\text {tot }}\right)$ to some value $R_{\text {prec, max }}$ obtained by requesting that $R_{\text {sub }}=1$ (limit case of a totally smoothed shock, all the compression is done in the precursor). For a pure hydrodynamical shock, $R_{\text {sub }} \rightarrow 1$ is equivalent to $M_{S, 1} \rightarrow 1$ [see Eq. (135)], 
although this is no longer true for a magnetized shock, when taking into account the pressure $P_{\mathrm{w}, 1}$ of waves [see Eq. (133)], that reduces the compressibility of the medium. Note that when $P_{\mathrm{w}, 1}^{\star}$ reaches $1 / 2$, a shock can no longer form.

For each value of $R_{\text {prec }}$, the couple $\left(f_{1}, U_{p}\right)$ will be accepted as a solution of the model if and only if $U_{p}\left(p_{\max }\right)=1$. Note that a solution may be found for more than one value of $R_{\text {prec }}$. For most of the parameter space, a single solution is found, but sometimes three solutions are found. One corresponds to a weakly modified shock, while the other two correspond to significantly modified shocks (see Blasi et al. 2005 and Amato et al. 2008). Multiple solutions for CR-modified shocks had already been observed before (using completely different methods, see Sect. 5.3) but their physical meaning is unclear. In reality only a single solution will be realized, it is commonly assumed that the others will be suppressed because they are not stable. At this point we should keep in mind that Blasi's NLDSA model is not time-dependent, and thus cannot describe how the modified shock structure progressively takes shape. This requires numerical simulations of the kind presented in the previous Sects. 5.4 and 5.5.

\section{Summary and conclusions}

This review addresses the numerical techniques developed in the community of high-energy astrophysics and high-energy lasers to investigate non-thermal particle acceleration and transport in magnetized turbulent flows. We first review the main theoretical frameworks developed for the study of particle acceleration in astrophysical flows: diffusive shock acceleration, shock drift and shock surfing processes, stochastic acceleration, and also provide a short survey of recent developments in the field of laser plasmas. We do not cover the process of shear acceleration, the reader can advantageously consult the work of Rieger and Duffy (2006) for further reference. We then detail the technical numerical techniques necessary to investigate problems which appear in the kinetic treatment of particle acceleration. We only give at this stage a short introduction on hybrid methods, the reader is referred to Lipatov (2002) for further details. Kinetic problems in cosmic ray physics can also be treated in the framework of Fokker-Planck or diffusion-convection equations. The Fokker-Planck model finds many astrophysical applications, from the study of cosmic ray transport in the Galaxy to the study of hot plasmas around compact objects. We then focus on recent developments in the theory of particle acceleration at collisionless nonrelativistic or relativistic shocks and in reconnection sites based on particle-in-cell and/or hybrid simulations. The final part of the review addresses large-scale particle transport and acceleration studies mostly in the magnetohydrodynamic approximation. We review the rapid developments of numerical techniques coupling MHD with the kinetic description of non-thermal components. We end with the developments made to find semi-analytical solutions of the diffusion-convection equation in the context of CR acceleration at shocks. For completeness, we recommend interested readers to consult some recent and complementary reports and monographs on particle-in-cell methods and Vlasov methods (Palmroth et al. 2018), hybrid methods (Lipatov 2002), and magnetohydrodynamics (Martí and Müller 2015; Leveque 1998). 
The subject of energetic particle acceleration and transport in turbulent flows is rapidly growing thanks to the increase in computational power. This applies to standard techniques for catching the propagation of energetic particles like particle-in-cell simulations. Beside this, we have seen that the real challenge is to handle the dynamics over the space, time and energy scales of the high-energy phenomena in astrophysics. This is mandatory because as the highest energetic particles are accelerated they trigger magnetic perturbations necessary to the acceleration of lower energetic particles. This back-reaction requires numerical tools able to treat the inter-connections between large and micro scales. The recent effort in developing PIC modules in MHD codes goes in this direction. This is also true for magnetic reconnection. The microphysics of current sheets depends on the way the magnetic field lines are forced to reconnect by large scale motions. Here it seems important to have simulations which combine MHD and kinetic simulations. One major difficulty remains however to control the numerical noise inherently related to PIC simulations (either due to Cherenkov radiation, or as in the case of PIC-MHD method due to the perturbations generated by the energetic particles themselves). An alternative resides in using a Vlasov approach (Lautenbach and Grauer 2018), but this possibility remains limited by numerical resources to investigate multi-dimensional problems properly. This aspect is crucial to a proper description of particle acceleration and turbulence around shocks and in reconnection zones. One way to make progress beyond the increase of computational power is to combine different numerical techniques to investigate different regions, as it is the case with implicit PIC simulations coupled with MHD solvers (Makwana et al. 2017; Rieke et al. 2015). Another challenge is to adapt the simulations developed for Newtonian flows to special and now general relativistic cases. This aspect is of particular importance since the era of multi-messenger astrophysics is now a reality, in a near future we will obtain an unprecedentedly accurate description of high-energy particle sources with the advent of high precision/high sensitivity gravitational wave, neutrino and gamma-ray detectors.

A positive aspect we can see is that the different numerical tools discussed in this review are and will be further routinely used by very different communities, i.e., highenergy astrophysics, high-energy laboratory plasmas and space plasmas to study the energetic events from the Sun. These converging interests will undoubtedly contribute to the emergence of new fruitful interdisciplinary research subjects.

Acknowledgements The authors thank Fabien Casse and Laurent Gremillet for several fruitful discussions. RW thanks Mickaël Melzani who contributed a lot to his knowledge of magnetic reconnection and Doris Folini for profound discussions and proof-reading. AM and MG thank Anna Grassi for her important contributions to Sects. 2.6 and 3.1-3.4. MG also thank Simon Bolanos for his input on magnetic reconnection experiments (Sect. 2.6.4), Francesco Califano, Daniele Del Sarto and Alain Ghizzo for various discussions on Vlasov codes (Sect. 3.4) and the SMILEI dev-team for various discussions on the PIC method. This work is supported by the ANR-14-CE33-0019 MACH project. We acknowledge support by the Programme National de Haute Énergie (PNHE).

Open Access This article is licensed under a Creative Commons Attribution 4.0 International License, which permits use, sharing, adaptation, distribution and reproduction in any medium or format, as long as you give appropriate credit to the original author(s) and the source, provide a link to the Creative Commons licence, and indicate if changes were made. The images or other third party material in this article are included in the article's Creative Commons licence, unless indicated otherwise in a credit line to the material. If material is not included in the article's Creative Commons licence and your intended use is not permitted 
by statutory regulation or exceeds the permitted use, you will need to obtain permission directly from the copyright holder. To view a copy of this licence, visit http://creativecommons.org/licenses/by/4.0/.

\section{References}

Achterberg A (1990) Particle acceleration by an ensemble of shocks. A\&A 231:251-258

Achterberg A, Schure KM (2011) A more accurate numerical scheme for diffusive shock acceleration. MNRAS 411:2628-2636. https://doi.org/10.1111/j.1365-2966.2010.17868.x. arXiv:1103.3049

Achterberg A, Gallant YA, Kirk JG, Guthmann AW (2001) Particle acceleration by ultrarelativistic shocks: theory and simulations. MNRAS 328:393-408. https://doi.org/10.1046/j.1365-8711.2001.04851.x. arXiv:astro-ph/0107530

Ahmed H, Dieckmann ME, Romagnani L, Doria D, Sarri G, Cerchez M, Ianni E, Kourakis I, Giesecke AL, Notley M, Prasad R, Quinn K, Willi O, Borghesi M (2013) Time-resolved characterization of the formation of a collisionless shock. Phys Rev Lett 110:205001. https://doi.org/10.1103/PhysRevLett. 110.205001

Amano T, Hoshino M (2007) Electron injection at high Mach number quasi-perpendicular shocks: surfing and drift acceleration. ApJ 661:190-202. https://doi.org/10.1086/513599. arXiv:astro-ph/0612204

Amano T, Hoshino M (2009) Electron shock surfing acceleration in multidimensions: two-dimensional particle-in-cell simulation of collisionless perpendicular shock. ApJ 690:244-251. https://doi.org/10. 1088/0004-637X/690/1/244. arXiv:0805.1098

Amato E, Blasi P (2005) A general solution to non-linear particle acceleration at nonrelativistic shock waves. MNRAS 364:L76-L80. https://doi.org/10.1111/j.1745-3933.2005.00110. x. arXiv:astro-ph/0509673

Amato E, Blasi P, Gabici S (2008) Kinetic approaches to particle acceleration at cosmic ray modified shocks. MNRAS 385:1946-1958. https://doi.org/10.1111/j.1365-2966.2008.12876.x. arXiv:0705.3723

Arber TD, Bennett K, Brady CS, Lawrence-Douglas A, Ramsay MG, Sircombe NJ, Gillies P, Evans RG, Schmitz H, Bell AR, Ridgers CP (2015) Contemporary particle-in-cell approach to laser-plasma modelling. Plasma Phys Control Fusion 57:113001. https://doi.org/10.1088/0741-3335/57/11/113001

Ardaneh K, Cai D, Nishikawa KI, Lembége B (2015) Collisionless Weibel shocks and electron acceleration in gamma-ray bursts. ApJ 811:57. https://doi.org/10.1088/0004-637X/811/1/57. arXiv:1507.05374

Ashour-Abdalla M, Lapenta G, Walker RJ, El-Alaoui M, Liang H (2015) Multiscale study of electron energization during unsteady reconnection events. J Geophys Res 120:4784-4799. https://doi.org/10. 1002/2014JA020316

Atoyan AM, Aharonian FA (1996) On the fluxes of inverse Compton gamma-rays expected from the Crab Nebula. A\&AS 120:453-456

Atzeni S, Meyer-Ter-Vehn J (2004) The physics of inertial fusion: beam plasma interaction, hydrodynamics, hot dense matter. Oxford University Press, Oxford. https://doi.org/10.1093/acprof:oso/ 9780198562641.001 .0001

Aunai N, Hesse M, Zenitani S, Kuznetsova M, Black C, Evans R, Smets R (2013) Comparison between hybrid and fully kinetic models of asymmetric magnetic reconnection: Coplanar and guide field configurations. Phys Plasmas 20:022902. https://doi.org/10.1063/1.4792250. arXiv:1302.0506

Axford WI, Leer E, Skadron G (1977) The acceleration of cosmic rays by shock waves. In: Christov CY (ed) 15th international cosmic ray conference, vol 11. Bulgarian Academy of Sciences, Sofia, pp 132-137

Bader M (2013) Space-filling curves: an introduction with applications in scientific computing. Texts in Computational Science and Engineering, vol 9. Springer, Berlin. https://doi.org/10.1007/978-3-64231046-1

Bai XN, Caprioli D, Sironi L, Spitkovsky A (2015) Magnetohydrodynamic-particle-in-cell method for coupling cosmic rays with a thermal plasma: application to non-relativistic shocks. ApJ 809:55. https:// doi.org/10.1088/0004-637X/809/1/55. arXiv:1412.1087

Bai XN, Ostriker EC, Plotnikov I, Stone JM (2019) Magnetohydrodynamic particle-in-cell simulations of the cosmic-ray streaming instability: linear growth and quasi-linear evolution. ApJ 876(1):60. https:// doi.org/10.3847/1538-4357/ab1648. arXiv:1902.10219

Ball D, Sironi L, Özel F (2018) Electron and proton acceleration in trans-relativistic magnetic reconnection: dependence on plasma beta and magnetization. ApJ 862:80. https://doi.org/10.3847/1538-4357/ aac820. arXiv: 1803.05556 
Balsara DS (1998) Total variation diminishing scheme for adiabatic and isothermal magnetohydrodynamics. ApJS 116:133-153. https://doi.org/10.1086/313093

Balsara DS, Tilley DA, Howk JC (2008) Simulating anisotropic thermal conduction in supernova remnantsI. Numerical methods. MNRAS 386:627-641. https://doi.org/10.1111/j.1365-2966.2008.13085.x

Baumann G, Nordlund $\AA$ (2012) Particle-in-cell simulation of electron acceleration in solar coronal jets. ApJ 759:L9. https://doi.org/10.1088/2041-8205/759/1/L9. arXiv:1205.3486

Bednarz J, Ostrowski M (1998) Energy spectra of cosmic rays accelerated at ultrarelativistic shock waves. Phys Rev Lett 80:3911-3914. https://doi.org/10.1103/PhysRevLett.80.3911. arXiv:astro-ph/9806181

Begelman MC, Kirk JG (1990) Shock-drift particle acceleration in superluminal shocks: a model for hot spots in extragalactic radio sources. ApJ 353:66-80. https://doi.org/10.1086/168590

Bell AR (1978a) The acceleration of cosmic rays in shock fronts. I. MNRAS 182:147-156. https://doi.org/ 10.1093/mnras/182.2.147

Bell AR (1978b) The acceleration of cosmic rays in shock fronts. II. MNRAS 182:443-455. https://doi. org/10.1093/mnras/182.3.443

Bell AR (1987) The non-linear self-regulation of cosmic ray acceleration at shocks. MNRAS 225:615-626. https://doi.org/10.1093/mnras/225.3.615

Bell AR (2004) Turbulent amplification of magnetic field and diffusive shock acceleration of cosmic rays. MNRAS 353:550-558. https://doi.org/10.1111/j.1365-2966.2004.08097.x

Bell AR (2005) The interaction of cosmic rays and magnetized plasma. MNRAS 358:181-187. https://doi. org/10.1111/j.1365-2966.2005.08774.x

Bell AR (2008) Cosmic ray acceleration by a supernova shock in a dense circumstellar plasma. MNRAS 385:1884-1892. https://doi.org/10.1111/j.1365-2966.2008.13011.x

Bell AR (2013) Cosmic ray acceleration. Astropart Phys 43:56-70. https://doi.org/10.1016/j.astropartphys. 2012.05.022

Bell AR, Lucek SG (2001) Cosmic ray acceleration to very high energy through the non-linear amplification by cosmic rays of the seed magnetic field. MNRAS 321:433-438. https://doi.org/10.1046/j.13658711.2001.04063.x

Bell AR, Choi P, Dangor AE, Willi O, Bassett DA, Hooker CJ (1988) Collisionless shock in a laser-produced ablating plasma. Phys Rev A 38:1363-1369. https://doi.org/10.1103/PhysRevA.38.1363

Bell AR, Robinson APL, Sherlock M, Kingham RJ, Rozmus W (2006) Fast electron transport in laserproduced plasmas and the KALOS code for solution of the Vlasov-Fokker-Planck equation. Plasma Phys Control Fusion 48:R37-R57. https://doi.org/10.1088/0741-3335/48/3/R01

Bell AR, Schure KM, Reville B (2011) Cosmic ray acceleration at oblique shocks. MNRAS 418:1208-1216. https://doi.org/10.1111/j.1365-2966.2011.19571.x. arXiv:1108.0582

Bell AR, Schure KM, Reville B, Giacinti G (2013) Cosmic-ray acceleration and escape from supernova remnants. MNRAS 431:415-429. https://doi.org/10.1093/mnras/stt179. arXiv:1301.7264

Belmont R, Malzac J, Marcowith A (2008) Simulating radiation and kinetic processes in relativistic plasmas. A\&A 491:617-631. https://doi.org/10.1051/0004-6361:200809982. arXiv:0808.1258

Belmont G, Aunai N, Smets R (2012) Kinetic equilibrium for an asymmetric tangential layer. Phys Plasmas 19:022108. https://doi.org/10.1063/1.3685707

Beloborodov AM (2017) Radiative magnetic reconnection near accreting black holes. ApJ 850:141. https:// doi.org/10.3847/1538-4357/aa8f4f. arXiv:1701.02847

Beresnyak A, Li H (2014) Basic Bell-MHD turbulence. ApJ 788:107. https://doi.org/10.1088/0004-637X/ 788/2/107. arXiv:1406.1186

Berezhko EG (1996) Maximum energy of cosmic rays accelerated by supernova shocks. Astropart Phys 5:367-378. https://doi.org/10.1016/0927-6505(96)00037-0

Berezhko EG, Ellison DC (1999) A simple model of nonlinear diffusive shock acceleration. ApJ 526:385399. https://doi.org/10.1086/307993

Berezhko EG, Völk HJ (1997) Kinetic theory of cosmic rays and gamma rays in supernova remnants. I. Uniform interstellar medium. Astropart Phys 7:183-202. https://doi.org/10.1016/S0927-6505(97)000169

Berger MJ, Oliger J (1984) Adaptive mesh refinement for hyperbolic partial differential equations. J Comput Phys 53:484-512. https://doi.org/10.1016/0021-9991(84)90073-1

Bessho N, Bhattacharjee A (2012) Fast magnetic reconnection and particle acceleration in relativistic lowdensity electron-positron plasmas without guide field. ApJ 750:129. https://doi.org/10.1088/0004$637 \mathrm{X} / 750 / 2 / 129$ 
Birdsall CK, Fuss D (1969) Clouds-in-clouds, clouds-in-cells physics for many-body plasma simulation. J Comput Phys 3:494-511. https://doi.org/10.1016/0021-9991(69)90058-8

Birdsall CK, Langdon AB (1985) Plasma physics via computer simulation. McGraw-Hill, New York

Birn J, Priest ER (eds) (2007) Reconnection of magnetic fields: magnetohydrodynamics and collisionless theory and observations. Cambridge University Press, Cambridge

Biskamp D (2000) Magnetic reconnection in plasmas, Cambridge Monographs on Plasma Physics, vol 3. Cambridge University Press, Cambridge

Biskamp D, Welter H (1972) Ion heating in high-Mach-number, oblique, collisionless shock waves. Phys Rev Lett 28:410-413. https://doi.org/10.1103/PhysRevLett.28.410

Blackburn TG, Marklund M (2018) Nonlinear Breit-Wheeler pair creation with bremsstrahlung $\gamma$ rays. Plasma Phys Control Fusion 60:054009. https://doi.org/10.1088/1361-6587/aab3b4. arXiv:1802.06612

Blandford RD (1994) Particle acceleration mechanisms. ApJS 90:515-520. https://doi.org/10.1086/191869

Blandford RD, Ostriker JP (1978) Particle acceleration by astrophysical shocks. ApJ 221:L29-L32. https:// doi.org/10.1086/182658

Blasi P (2002) A semi-analytical approach to non-linear shock acceleration. Astropart Phys 16:429-439. https://doi.org/10.1016/S0927-6505(01)00127-X. arXiv:astro-ph/0104064

Blasi P (2004) Nonlinear shock acceleration in the presence of seed particles. Astropart Phys 21:45-57. https://doi.org/10.1016/j.astropartphys.2003.10.008. arXiv:astro-ph/0310507

Blasi P, Gabici S, Vannoni G (2005) On the role of injection in kinetic approaches to non-linear particle acceleration at non-relativistic shock waves. MNRAS 361:907-918. https://doi.org/10.1111/j.13652966.2005.09227.x. arXiv:astro-ph/0505351

Boeuf JP, Garrigues L (2018) Exb electron drift instability in hall thrusters: particle-in-cell simulations vs theory. Phys Plasmas 25:061204. https://doi.org/10.1063/1.5017033

Bohdan A, Niemiec J, Kobzar O, Pohl M (2017) Electron pre-acceleration at nonrelativistic high-Mach-number perpendicular shocks. ApJ 847:71. https://doi.org/10.3847/1538-4357/aa872a. arXiv: 1708.05528

Borghesi M, Schiavi A, Campbell DH, Haines MG, Willi O, MacKinnon AJ, Gizzi LA, Galimberti M, Clarke RJ, Ruhl H (2001) Proton imaging: a diagnostic for inertial confinement fusion/fast ignitor studies. Plasma Phys Control Fusion 43:A267-A276. https://doi.org/10.1088/0741-3335/43/12a/320

Bosch-Ramon V (2012) Fermi I particle acceleration in converging flows mediated by magnetic reconnection. A\&A 542:A125. https://doi.org/10.1051/0004-6361/201219231. arXiv:1205.3450

Bouchut F (2004) Nonlinear stability of finite volume methods for hyperbolic conservation laws, and wellbalanced schemes for sources. Front Math. https://doi.org/10.1007/b93802

Brackbill JU, Barnes DC (1980) The effect of nonzero product of magnetic gradient and B on the numerical solution of the magnetohydrodynamic equations. J Comput Phys 35:426-430. https://doi.org/10.1016/ 0021-9991(80)90079-0

Brandenburg A (2018) Advances in mean-field dynamo theory and applications to astrophysical turbulence. J Plasma Phys 84:735840404. https://doi.org/10.1017/S0022377818000806. arXiv:1801.05384

Breitschwerdt D, McKenzie JF, Voelk HJ (1991) Galactic winds. I. Cosmic ray and wave-driven winds from the Galaxy. A\&A 245:79-98

Breitschwerdt D, McKenzie JF, Voelk HJ (1993) Galactic winds. II. Role of the disk-halo interface in cosmic ray driven galactic winds. A\&A 269:54-66

Bret A (2009) Weibel, two-stream, filamentation, oblique, Bell, Buneman...Which one grows faster? ApJ 699:990-1003. https://doi.org/10.1088/0004-637X/699/2/990. arXiv:0903.2658

Bret A, Stockem A, Fiuza F, Ruyer C, Gremillet L, Narayan R, Silva LO (2013) Relativistic collisionless shocks formation in pair plasmas. J Plasma Phys 79(4):367-370. https://doi.org/10.1017/ S0022377813000354

Bret A, Stockem A, Narayan R, Silva LO (2014) Collisionless Weibel shocks: full formation mechanism and timing. Phys Plasmas 21:072301. https://doi.org/10.1063/1.4886121. arXiv:1406.4144

Brio M, Wu CC (1988) An upwind differencing scheme for the equations of ideal magnetohydrodynamics. J Comput Phys 75:400-422. https://doi.org/10.1016/0021-9991(88)90120-9

Brown PN, Hindmarsh AC (1989) Reduced storage matrix methods in stiff ode systems. Appl Math Comput 31:40-91. https://doi.org/10.1016/0096-3003(89)90110-0

Brunetti G, Lazarian A (2007) Compressible turbulence in galaxy clusters: physics and stochastic particle re-acceleration. MNRAS 378:245-275. https://doi.org/10.1111/j.1365-2966.2007.11771.x. arXiv:astro-ph/0703591 
Bryan GL, Norman ML, O’Shea BW, Abel T, Wise JH, Turk MJ, Reynolds DR, Collins DC, Wang P, Skillman SW, Smith B, Harkness RP, Bordner J, Kim Jh, Kuhlen M, Xu H, Goldbaum N, Hummels C, Kritsuk AG, Tasker E, Skory S, Simpson CM, Hahn O, Oishi JS, So GC, Zhao F, Cen R, Li Y, Collaboration Enzo (2014) ENZO: an adaptive mesh refinement code for astrophysics. ApJS 211:19. https://doi.org/10.1088/0067-0049/211/2/19. arXiv:1307.2265

Bucciantini N, Arons J, Amato E (2011) Modelling spectral evolution of pulsar wind nebulae inside supernova remnants. MNRAS 410:381-398. https://doi.org/10.1111/j.1365-2966.2010.17449. X. arXiv:1005.1831

Büchner J (2007) Vlasov-code simulation. In: Usui H, Omura Y (eds) Advanced methods for space simulations. TERRAPUB, Tokyo, pp 23-46

Bühler R, Blandford R (2014) The surprising Crab pulsar and its nebula: a review. Rep Prog Phys 77:066901. https://doi.org/10.1088/0034-4885/77/6/066901. arXiv:1309.7046

Buneman O (1959) Dissipation of currents in ionized media. Phys Rev 115:503-517. https://doi.org/10. 1103/PhysRev.115.503

Buneman O (1993) TRISTAN: the 3-d electromagnetic particle code. In: Matsumoto H, Omura Y (eds) Computer space plasma physics: simulation techniques and software. Terra Scientific, Tokyo, pp 67-84

Butsky IS, Quinn TR (2018) The role of cosmic-ray transport in shaping the simulated circumgalactic medium. ApJ 868:108. https://doi.org/10.3847/1538-4357/aaeac2. arXiv:1803.06345

Bykov AM, Fleishman GD (1992) On non-thermal particle generation in superbubbles. MNRAS 255:269275. https://doi.org/10.1093/mnras/255.2.269

Bykov AM, Toptygin IN (1987) Effect of shocks on interstellar turbulence and cosmic-ray dynamics. Ap\&SS 138:341-354. https://doi.org/10.1007/BF00637855

Bykov AM, Treumann RA (2011) Fundamentals of collisionless shocks for astrophysical application, 2. Relativistic shocks. A\&AR 19:42. https://doi.org/10.1007/s00159-011-0042-8. arXiv:1105.3221

Bykov AM, Pavlov GG, Artemyev AV, Uvarov YA (2012) Twinkling pulsar wind nebulae in the synchrotron cut-off regime and the $\gamma$-ray flares in the Crab Nebula. MNRAS 421:L67-L71. https://doi.org/10.1111/ j.1745-3933.2011.01208.x. arXiv:1112.3114

Bykov AM, Brandenburg A, Malkov MA, Osipov SM (2013) Microphysics of cosmic ray driven plasma instabilities. Space Sci Rev 178:201-232. https://doi.org/10.1007/s11214-013-9988-3. arXiv: 1304.7081

Califano F, Lontano M (2003) Vlasov-maxwell simulations of high-frequency longitudinal waves in a magnetized plasma. Phys Rev E 67:056401. https://doi.org/10.1103/PhysRevE.67.056401

Califano F, Cecchi T, Chiuderi C (2002) Nonlinear kinetic regime of the Weibel instability in an electron-ion plasma. Phys Plasmas 9:451-457. https://doi.org/10.1063/1.1435001

Candia J, Roulet E (2004) Diffusion and drift of cosmic rays in highly turbulent magnetic fields. J Cosmol Astropart Phys 10:007. https://doi.org/10.1088/1475-7516/2004/10/007. arXiv:astro-ph/0408054

Caprioli D, Spitkovsky A (2014a) Simulations of ion acceleration at non-relativistic shocks. I. Acceleration efficiency. ApJ 783:91. https://doi.org/10.1088/0004-637X/783/2/91. arXiv:1310.2943

Caprioli D, Spitkovsky A (2014b) Simulations of Ion acceleration at non-relativistic shocks. II. Magnetic field amplification. ApJ 794:46. https://doi.org/10.1088/0004-637X/794/1/46. arXiv:1401.7679

Caprioli D, Blasi P, Amato E, Vietri M (2008) Dynamical effects of self-generated magnetic fields in cosmic-ray-modified shocks. ApJ 679:L139. https://doi.org/10.1086/589505. arXiv:0804.2884

Caprioli D, Blasi P, Amato E (2009a) On the escape of particles from cosmic ray modified shocks. MNRAS 396:2065-2073. https://doi.org/10.1111/j.1365-2966.2008.14298.x. arXiv:0807.4259

Caprioli D, Blasi P, Amato E, Vietri M (2009b) Dynamical feedback of self-generated magnetic fields in cosmic ray modified shocks. MNRAS 395:895-906. https://doi.org/10.1111/j.1365-2966.2009. 14570.x. arXiv:0807.4261

Caprioli D, Amato E, Blasi P (2010a) Non-linear diffusive shock acceleration with free-escape boundary. Astropart Phys 33:307-311. https://doi.org/10.1016/j.astropartphys.2010.03.001. arXiv:0912.2714

Caprioli D, Kang H, Vladimirov AE, Jones TW (2010b) Comparison of different methods for nonlinear diffusive shock acceleration. MNRAS 407:1773-1783. https://doi.org/10.1111/j.1365-2966. 2010.17013.x. arXiv:1005.2127

Caprioli D, Pop AR, Spitkovsky A (2015) Simulations and theory of ion injection at non-relativistic collisionless shocks. ApJ 798:L28. https://doi.org/10.1088/2041-8205/798/2/L28. arXiv:1409.8291

Caprioli D, Yi DT, Spitkovsky A (2017) Chemical enhancements in shock-accelerated particles: ab initio simulations. Phys Rev Lett 119:171101. https://doi.org/10.1103/PhysRevLett.119.171101. arXiv: 1704.08252 
Caprioli D, Zhang H, Spitkovsky A (2018) Diffusive shock re-acceleration. J Plasma Phys 84:715840301. https://doi.org/10.1017/S0022377818000478. arXiv:1801.01510

Cassak PA, Shay MA (2007) Scaling of asymmetric magnetic reconnection: general theory and collisional simulations. Phys Plasmas 14:102114. https://doi.org/10.1063/1.2795630

Casse F, Marcowith A (2003) Relativistic particle transport in extragalactic jets. I. Coupling MHD and kinetic theory. A\&A 404:405-421. https://doi.org/10.1051/0004-6361:20030547. arXiv:astro-ph/0303579

Casse F, Marcowith A (2005) Astroparticle yield and transport from extragalactic jet terminal shocks. Astropart Phys 23:31-56. https://doi.org/10.1016/j.astropartphys.2004.11.003. arXiv:astro-ph/0411395

Casse F, Lemoine M, Pelletier G (2002) Transport of cosmic rays in chaotic magnetic fields. Phys Rev D 65:023002. https://doi.org/10.1103/PhysRevD.65.023002. arXiv:astro-ph/0109223

Casse F, Marcowith A, Keppens R (2013) Non-resonant magnetohydrodynamics streaming instability near magnetized relativistic shocks. MNRAS 433:940-951. https://doi.org/10.1093/mnras/stt772. arXiv: 1305.0847

Cerri SS, Franci L, Califano F, Landi S, Hellinger P (2017) Plasma turbulence at ion scales: a comparison between particle in cell and Eulerian hybrid-kinetic approaches. J Plasma Phys 83:705830202. https:// doi.org/10.1017/S0022377817000265

Cerri SS, Kunz MW, Califano F (2018) Dual phase-space cascades in 3D hybrid-Vlasov-Maxwell turbulence. Astrophys J 856:L13. https://doi.org/10.3847/2041-8213/aab557

Cerutti B, Beloborodov AM (2017) Electrodynamics of pulsar magnetospheres. Space Sci Rev 207:111136. https://doi.org/10.1007/s11214-016-0315-7. arXiv:1611.04331

Cerutti B, Philippov AA (2017) Dissipation of the striped pulsar wind. A\&A 607:A134. https://doi.org/10. 1051/0004-6361/201731680. arXiv: 1710.07320

Cerutti B, Uzdensky DA, Begelman MC (2012a) Extreme particle acceleration in magnetic reconnection layers: application to the gamma-ray flares in the Crab Nebula. ApJ 746:148. https://doi.org/10.1088/ 0004-637X/746/2/148. arXiv: 1110.0557

Cerutti B, Werner GR, Uzdensky DA, Begelman MC (2012b) Beaming and rapid variability of high-energy radiation from relativistic pair plasma reconnection. ApJ 754:L33. https://doi.org/10.1088/2041-8205/ 754/2/L33. arXiv:1205.3210

Cerutti B, Werner GR, Uzdensky DA, Begelman MC (2013) Simulations of particle acceleration beyond the classical synchrotron burnoff limit in magnetic reconnection: an explanation of the Crab flares. ApJ 770:147. https://doi.org/10.1088/0004-637X/770/2/147. arXiv:1302.6247

Cerutti B, Werner GR, Uzdensky DA, Begelman MC (2014a) Gamma-ray flares in the Crab Nebula: a case of relativistic reconnection? Phys Plasmas 21:056501. https://doi.org/10.1063/1.4872024. arXiv: 1401.3016

Cerutti B, Werner GR, Uzdensky DA, Begelman MC (2014b) Three-dimensional relativistic pair plasma reconnection with radiative feedback in the Crab Nebula. ApJ 782:104. https://doi.org/10.1088/0004637X/782/2/104. arXiv:1311.2605

Cerutti B, Philippov AA, Spitkovsky A (2016) Modelling high-energy pulsar light curves from first principles. MNRAS 457:2401-2414. https://doi.org/10.1093/mnras/stw124. arXiv:1511.01785

Chang JS, Cooper G (1970) A practical difference scheme for Fokker-Planck equations. J Comput Phys 6:1-16. https://doi.org/10.1016/0021-9991(70)90001-X

Chang P, Spitkovsky A, Arons J (2008) Long-term evolution of magnetic turbulence in relativistic collisionless shocks: electron-positron plasmas. ApJ 674:378-387. https://doi.org/10.1086/524764. arXiv:0704.3832

Chen H, Fiuza F, Link A, Hazi A, Hill M, Hoarty D, James S, Kerr S, Meyerhofer DD, Myatt J, Park J, Sentoku Y, Williams GJ (2015) Scaling the yield of laser-driven electron-positron jets to laboratory astrophysical applications. Phys Rev Lett 114:215001. https://doi.org/10.1103/PhysRevLett.114. 215001

Cheng CZ, Knorr G (1976) The integration of the Vlasov equation in configuration space. J Comput Phys 22:330-351. https://doi.org/10.1016/0021-9991(76)90053-X

Cheung AY, Goforth RR, Koopman DW (1973) Magnetically induced collisionless coupling between counterstreaming laser-produced plasmas. Phys Rev Lett 31:429-432. https://doi.org/10.1103/ PhysRevLett.31.429

Colella P (1990) Multidimensional upwind methods for hyperbolic conservation laws. J Comput Phys 87:171-200. https://doi.org/10.1016/0021-9991(90)90233-Q 
Colella P, Woodward PR (1984) The piecewise parabolic method (PPM) for gas-dynamical simulations. J Comput Phys 54:174-201. https://doi.org/10.1016/0021-9991(84)90143-8

Courant R, Friedrichs K, Lewy H (1928) Über die partiellen Differenzengleichungen der mathematischen Physik. Math Ann 100:32-74. https://doi.org/10.1007/BF01448839

Crouseilles N, Mehrenberger M, Sonnendrücker E (2010) Conservative semi-Lagrangian schemes for Vlasov equations. J Comput Phys 229:1927-1953. https://doi.org/10.1016/j.jcp.2009.11.007

Crumley P, Caprioli D, Markoff S, Spitkovsky A (2019) Kinetic simulations of mildly relativistic shocks-I. Particle acceleration in high Mach number shocks. MNRAS 485:5105-5119. https://doi.org/10.1093/ mnras/stz232. arXiv:1809.10809

Daldorff LKS, Tóth G, Gombosi TI, Lapenta G, Amaya J, Markidis S, Brackbill JU (2014) Two-way coupling of a global Hall magnetohydrodynamics model with a local implicit particle-in-cell model. J Comput Phys 268:236-254. https://doi.org/10.1016/j.jcp.2014.03.009

Daughton W, Roytershteyn V (2012) Emerging parameter space map of magnetic reconnection in collisional and kinetic regimes. Space Sci Rev 172:271-282. https://doi.org/10.1007/s11214-011-9766-Z

Daughton W, Roytershteyn V, Karimabadi H, Yin L, Albright BJ, Bergen B, Bowers KJ (2011) Role of electron physics in the development of turbulent magnetic reconnection in collisionless plasmas. Nature Phys 7:539-542. https://doi.org/10.1038/nphys 1965

Daughton W, Nakamura TKM, Karimabadi H, Roytershteyn V, Loring B (2014) Computing the reconnection rate in turbulent kinetic layers by using electron mixing to identify topology. Phys Plasmas 21:052307. https://doi.org/10.1063/1.4875730

Dawson J (1962) One-dimensional plasma model. Phys Fluids 5:445-459. https://doi.org/10.1063/1. 1706638

Dawson JM (1983) Particle simulation of plasmas. Rev Mod Phys 55:403-447. https://doi.org/10.1103/ RevModPhys.55.403

de Gouveia Dal Pino EM, Kowal G (2015) Particle Acceleration by Magnetic Reconnection. In: Lazarian A, de Gouveia Dal Pino EM, Melioli C (eds) Magnetic fields in diffuse media. Astrophysics and Space Science Library, vol. 407. Springer, Berlin, Heidelberg, p 373. https://doi.org/10.1007/978-3-66244625-6_13. arXiv:1302.4374

de Gouveia dal Pino EM, Lazarian A (2005) Production of the large scale superluminal ejections of the microquasar GRS $1915+105$ by violent magnetic reconnection. A\&A 441:845-853. https://doi.org/ 10.1051/0004-6361:20042590

Dean SO, McLean EA, Stamper JA, Griem HR (1971) Demonstration of collisionless interactions between interstreaming ions in a laser-produced-plasma experiment. Phys Rev Lett 27:487-490. https://doi. org/10.1103/PhysRevLett.27.487

Dean SO, McLean EA, Stamper JA, Griem HR (1972) Reasons for the collisionless nature of interactions in a laser-produced plasma experiment. Phys Rev Lett 29:569-573. https://doi.org/10.1103/PhysRevLett. 29.569

Decker RB (1988) Computer modeling of test particle acceleration at oblique shocks. Space Sci Rev 48:195-262. https://doi.org/10.1007/BF00226009

Decker RB, Vlahos L (1985) Shock drift acceleration in the presence of waves. J Geophys Res 90:47-56. https://doi.org/10.1029/JA090iA01p00047

Decourchelle A, Ellison DC, Ballet J (2000) Thermal X-ray emission and cosmic-ray production in young supernova remnants. ApJ 543:L57-L60. https://doi.org/10.1086/318167. arXiv:astro-ph/0008344

Dedner A, Kemm F, Kröner D, Munz CD, Schnitzer T, Wesenberg M (2002) Hyperbolic divergence cleaning for the MHD equations. J Comput Phys 175:645-673. https://doi.org/10.1006/jcph.2001.6961

Del Santo M, Malzac J, Belmont R, Bouchet L, De Cesare G (2013) The magnetic field in the X-ray corona of Cygnus X-1. MNRAS 430:209-220. https://doi.org/10.1093/mnras/sts574. arXiv:1212.2040

Del Zanna L, Bucciantini N, Londrillo P (2003) An efficient shock-capturing central-type scheme for multidimensional relativistic flows. II. Magnetohydrodynamics. A\&A 400:397-413. https://doi.org/ 10.1051/0004-6361:20021641. arXiv:astro-ph/0210618

Del Zanna L, Papini E, Landi S, Bugli M, Bucciantini N (2016) Fast reconnection in relativistic plasmas: the magnetohydrodynamics tearing instability revisited. MNRAS 460:3753-3765. https://doi.org/10. 1093/mnras/stw1242. arXiv:1605.06331

Delamere PA, Bagenal F, Paranicas C, Masters A, Radioti A, Bonfond B, Ray L, Jia X, Nichols J, Arridge C (2015) Solar wind and internally driven dynamics: influences on magnetodiscs and auroral responses. Space Sci Rev 187:51-97. https://doi.org/10.1007/s11214-014-0075-1 
Dermer CD, Miller JA, Li H (1996) Stochastic particle acceleration near accreting black holes. ApJ 456:106. https://doi.org/10.1086/176631. arXiv:astro-ph/9508069

Derouillat J, Beck A, Pérez F, Vinci T, Chiaramello M, Grassi A, Flé M, Bouchard G, Plotnikov I, Aunai N, Dargent J, Riconda C, Grech M (2018) Smilei : a collaborative, open-source, multi-purpose particlein-cell code for plasma simulation. Comput Phys Commun 222:351-373. https://doi.org/10.1016/j. cpc.2017.09.024

Di Matteo T (1998) Magnetic reconnection: flares and coronal heating in active galactic nuclei. MNRAS 299:L15-120. https://doi.org/10.1046/j.1365-8711.1998.01950.x. arXiv:astro-ph/9805347

Dieckmann ME, Bret A (2017) Simulation study of the formation of a non-relativistic pair shock. J Plasma Phys 83:905830104. https://doi.org/10.1017/S0022377816001288. arXiv:1701.04075

Dieckmann ME, Bret A (2018) Electrostatic and magnetic instabilities in the transition layer of a collisionless weakly relativistic pair shock. MNRAS 473:198-209. https://doi.org/10.1093/mnras/stx2387

Dieckmann ME, Frederiksen JT, Bret A, Shukla PK (2006) Evolution of the fastest-growing relativistic mixed mode instability driven by a tenuous plasma beam in one and two dimensions. Phys Plasmas 13:112110. https://doi.org/10.1063/1.2390687

Dieckmann ME, Shukla PK, Drury LOC (2008) The formation of a relativistic partially electromagnetic planar plasma shock. ApJ 675:586-595. https://doi.org/10.1086/525516. arXiv:astro-ph/0702055

Dieckmann ME, Bret A, Sarri G, Perez Alvaro E, Kourakis I, Borghesi M (2012) Particle simulation study of electron heating by counter-streaming ion beams ahead of supernova remnant shocks. Plasma Phys Control Fusion 54:085015. https://doi.org/10.1088/0741-3335/54/8/085015. arXiv:1206.5713

Dieckmann ME, Alejo A, Sarri G (2018a) Expansion of a mildly relativistic hot pair cloud into an electronproton plasma. Phys Plasmas 25:062122. https://doi.org/10.1063/1.5036954. arXiv:1805.10447

Dieckmann ME, Alejo A, Sarri G, Folini D, Walder R (2018b) One-dimensional thermal pressure-driven expansion of a pair cloud into an electron-proton plasma. Phys Plasmas 25:064502. https://doi.org/ 10.1063/1.5026568. arXiv: 1805.08422

Dieckmann ME, Folini D, Hotz I, Nordman A, Dell'Acqua P, Ynnerman A, Walder R (2019) Structure of a collisionless pair jet in a magnetized electron-proton plasma: flow-aligned magnetic field. A\&A 621:A142. https://doi.org/10.1051/0004-6361/201834393

Dong QL, Wang SJ, Lu QM, Huang C, Yuan DW, Liu X, Lin XX, Li YT, Wei HG, Zhong JY (2012) Plasmoid ejection and secondary current sheet generation from magnetic reconnection in laser-plasma interaction. Phys Rev Lett 108:215001. https://doi.org/10.1103/PhysRevLett.108.215001. arXiv:1203.4036

Donnert J, Brunetti G (2014) An efficient Fokker-Planck solver and its application to stochastic particle acceleration in galaxy clusters. MNRAS 443:3564-3577. https://doi.org/10.1093/mnras/stu1417. arXiv: 1407.2735

Dosch A, Shalchi A (2010) Diffusive shock acceleration at interplanetary perpendicular shock waves: influence of the large scale structure of turbulence on the maximum particle energy. Adv Space Res 46:1208-1217. https://doi.org/10.1016/j.asr.2010.07.001

Drake RP, Gregori G (2012) Design considerations for unmagnetized collisionless-shock measurements in homologous flows. Astrophys J 749:171. https://doi.org/10.1088/0004-637x/749/2/171

Drake JF, Swisdak M, Che H, Shay MA (2006) Electron acceleration from contracting magnetic islands during reconnection. Nature 443:553-556. https://doi.org/10.1038/nature05116

Drake JF, Opher M, Swisdak M, Chamoun JN (2010) A magnetic reconnection mechanism for the generation of anomalous cosmic rays. ApJ 709:963-974. https://doi.org/10.1088/0004-637X/709/2/963. arXiv:0911.3098

Drury LO (1983) An introduction to the theory of diffusive shock acceleration of energetic particles in tenuous plasmas. Rep Prog Phys 46:973-1027. https://doi.org/10.1088/0034-4885/46/8/002

Drury LO (2012) First-order Fermi acceleration driven by magnetic reconnection. MNRAS 422:2474-2476. https://doi.org/10.1111/j.1365-2966.2012.20804.x. arXiv:1201.6612

Drury LO, Strong AW (2017) Power requirements for cosmic ray propagation models involving diffusive reacceleration; estimates and implications for the damping of interstellar turbulence. A\&A 597:A117. https://doi.org/10.1051/0004-6361/201629526. arXiv:1608.04227

Drury LO, Voelk JH (1981) Hydromagnetic shock structure in the presence of cosmic rays. ApJ 248:344351. https://doi.org/10.1086/159159

Dubois Y, Commerçon B (2016) An implicit scheme for solving the anisotropic diffusion of heat and cosmic rays in the RAMSES code. A\&A 585:A138. https://doi.org/10.1051/0004-6361/201527126. arXiv: 1509.07037 
Duclous R, Kirk JG, Bell AR (2011) Monte carlo calculations of pair production in high-intensity laserplasma interactions. Plasma Phys Control Fusion 53:015009

Duffy P (1992) The self-consistent acceleration of cosmic rays in modified shocks with Bohm-type diffusion. A\&A 262:281-294

Dungey JW (1958) The neutral point discharge theory of solar flares. A reply to Cowling's criticism. In: Lehnert B (ed) Electromagnetic phenomena in cosmical physics, IAU symposium, vol 6, p 135

Eastwood JP, Phan TD, Øieroset M, Shay MA, Malakit K, Swisdak M, Drake JF, Masters A (2013) Influence of asymmetries and guide fields on the magnetic reconnection diffusion region in collisionless space plasmas. Plasma Phys Control Fusion 55:124001. https://doi.org/10.1088/0741-3335/55/12/124001

Edmon PP, Kang H, Jones TW, Ma R (2011) Non-thermal radiation from type Ia supernova remnants. MNRAS 414:3521-3536. https://doi.org/10.1111/j.1365-2966.2011.18652.x. arXiv:1103.0963

Eichler D (1979) Particle acceleration in collisionless shocks-regulated injection and high efficiency. ApJ 229:419-423. https://doi.org/10.1086/156969

Eichler D (1984) On the theory of cosmic-ray-mediated shocks with variable compression ratio. ApJ 277:429-434. https://doi.org/10.1086/161711

Einfeldt B, Roe PL, Munz CD, Sjogreen B (1991) On Godunov-type methods near low densities. J Comput Phys 92:273-295. https://doi.org/10.1016/0021-9991(91)90211-3

Ellison DC, Double GP (2004) Diffusive shock acceleration in unmodified relativistic, oblique shocks. Astropart Phys 22:323-338. https://doi.org/10.1016/j.astropartphys.2004.08.005. arXiv:astro-ph/0408527

Ellison DC, Eichler D (1984) Monte Carlo shock-like solutions to the Boltzmann equation with collective scattering. ApJ 286:691-701. https://doi.org/10.1086/162644

Ellison DC, Decourchelle A, Ballet J (2004) Hydrodynamic simulation of supernova remnants including efficient particle acceleration. A\&A 413:189-201. https://doi.org/10.1051/0004-6361:20034073. arXiv:astro-ph/0308308

Ellison DC, Patnaude DJ, Slane P, Blasi P, Gabici S (2007) Particle acceleration in supernova remnants and the production of thermal and nonthermal radiation. ApJ 661:879-891. https://doi.org/10.1086/ 517518. arXiv:astro-ph/0702674

Ellison DC, Warren DC, Bykov AM (2013) Monte Carlo simulations of nonlinear particle acceleration in parallel trans-relativistic shocks. ApJ 776:46. https://doi.org/10.1088/0004-637X/776/1/46. arXiv: 1308.5114

Enßlin TA, Pfrommer C, Springel V, Jubelgas M (2007) Cosmic ray physics in calculations of cosmological structure formation. A\&A 473:41-57. https://doi.org/10.1051/0004-6361:20065294. arXiv:astro-ph/0603484

Eriksson S, ØIeroset M, Baker DN, Mouikis C, Vaivads A, Dunlop MW, RèMe H, Ergun RE, Balogh A (2004) Walén and slow-mode shock analyses in the near-Earth magnetotail in connection with a substorm onset on 27 August 2001. J Geophys Res 109:A10212. https://doi.org/10.1029/2004JA010534

Esirkepov TZ (2001) Exact charge conservation scheme for particle-in-cell simulation with an arbitrary form-factor. Comput Phys Commun 135:144-153. https://doi.org/10.1016/S0010-4655(00)00228-9

Evans CR, Hawley JF (1988) Simulation of magnetohydrodynamic flows-a constrained transport method. ApJ 332:659-677. https://doi.org/10.1086/166684

Everett JE, Zweibel EG, Benjamin RA, McCammon D, Rocks L, Gallagher JS III (2008) The Milky Way's kiloparsec-scale wind: a hybrid cosmic-ray and thermally driven outflow. ApJ 674:258-270. https:// doi.org/10.1086/524766. arXiv:0710.3712

Evoli C, Gaggero D, Vittino A, Di Bernardo G, Di Mauro M, Ligorini A, Ullio P, Grasso D (2017) Cosmic-ray propagation with DRAGON2: I. Numerical solver and astrophysical ingredients. J Cosmol Astropart Phys 2:015. https://doi.org/10.1088/1475-7516/2017/02/015. arXiv:1607.07886

Falle SAEG, Giddings JR (1987) Time-dependent cosmic ray modified shocks. MNRAS 225:399-423. https://doi.org/10.1093/mnras/225.2.399

Feix MR, Bertrand P (2005) A universal model: the vlasov equation. Transp Theor Stat Phys 34:7-62. https://doi.org/10.1080/00411450500253703

Fermi E (1949) On the origin of the cosmic radiation. Phys Rev 75:1169-1174. https://doi.org/10.1103/ PhysRev.75.1169

Fermi E (1954) Galactic magnetic fields and the origin of cosmic radiation. ApJ 119:1. https://doi.org/10. $1086 / 145789$

Ferrand G, Marcowith A (2010) On the shape of the spectrum of cosmic rays accelerated inside superbubbles. A\&A 510:A101. https://doi.org/10.1051/0004-6361/200913520. arXiv:0911.4457 
Ferrand G, Downes T, Marcowith A (2008) MARCOS, a numerical tool for the simulation of multiple time-dependent non-linear diffusive shock acceleration. MNRAS 383:41-56. https://doi.org/10.1111/ j.1365-2966.2007.12511.x. arXiv:0710.1939

Ferrand G, Decourchelle A, Ballet J, Teyssier R, Fraschetti F (2010) 3D simulations of supernova remnants evolution including non-linear particle acceleration. A\&A 509:L10. https://doi.org/10.1051/00046361/200913666. arXiv:0912.4886

Ferrand G, Danos RJ, Shalchi A, Safi-Harb S, Edmon P, Mendygral P (2014a) Cosmic ray acceleration at perpendicular shocks in supernova remnants. ApJ 792:133. https://doi.org/10.1088/0004-637X/792/ 2/133. arXiv: 1407.6728

Ferrand G, Decourchelle A, Safi-Harb S (2014b) Three-dimensional simulations of the non-thermal broadband emission from young supernova remnants including efficient particle acceleration. ApJ 789:49. https://doi.org/10.1088/0004-637X/789/1/49. arXiv:1405.0614

Fijalkow E (1999) A numerical solution to the Vlasov equation. Comput Phys Commun 116:319-328 DOI S0010-4655(98)00146-5

Fiksel G, Fox W, Bhattacharjee A, Barnak DH, Chang PY, Germaschewski K, Hu SX, Nilson PM (2014) Magnetic reconnection between colliding magnetized laser-produced plasma plumes. Phys Rev Lett 113:105003. https://doi.org/10.1103/PhysRevLett.113.105003

Filbet F, Sonnendrücker E (2003) Comparison of eulerian vlasov solvers. Comput Phys Commun 150:247266. https://doi.org/10.1016/S0010-4655(02)00694-X

Filbet F, Sonnendrücker E, Bertrand P (2001) Conservative numerical schemes for the Vlasov equation. J Comput Phys 172:166-187. https://doi.org/10.1006/jcph.2001.6818

Fiuza F, Fonseca RA, Tonge J, Mori WB, Silva LO (2012) Weibel-instability-mediated collisionless shocks in the laboratory with ultraintense lasers. Phys Rev Lett 108:235004. https://doi.org/10.1103/ PhysRevLett.108.235004

Fonseca RA, Silva LO, Tsung FS, Decyk VK, Lu W, Ren C, Mori WB, Deng S, Lee S, Katsouleas T, Adam JC (2002) OSIRIS: a three-dimensional, fully relativistic particle in cell code for modeling plasma based accelerators. In: Sloot PMA, Hoekstra AG, Tan CJK, Dongarra JJ (eds) Computational science-ICCS 2002. Lecture notes in computer science, vol 2331. Springer, Berlin, pp 342-351. https://doi.org/10.1007/3-540-47789-6_36

Fox W, Fiksel G, Bhattacharjee A, Chang PY, Germaschewski K, Hu SX, Nilson PM (2013) Filamentation instability of counterstreaming laser-driven plasmas. Phys Rev Lett 111:225002. https://doi.org/10. 1103/PhysRevLett.111.225002

Frank A, Jones TW, Ryu D (1995) Time-dependent simulation of oblique MHD cosmic-ray shocks using the two-fluid model. ApJ 441:629-643. https://doi.org/10.1086/175388

Frederiksen JT, Hededal CB, Haugbølle T, Nordlund $\AA$ (2004) Magnetic field generation in collisionless shocks: pattern growth and transport. ApJ 608:L13-L16. https://doi.org/10.1086/421262. arXiv:astro-ph/0308104

Fried BD (1959) Mechanism for instability of transverse plasma waves. Phys Fluids 2:337-337. https://doi. org/10.1063/1.1705933

Gallant YA, Achterberg A (1999) Ultra-high-energy cosmic ray acceleration by relativistic blast waves. MNRAS 305:L6-L10. https://doi.org/10.1046/j.1365-8711.1999.02566.x. arXiv:astro-ph/9812316

Gallant YA, Hoshino M, Langdon AB, Arons J, Max CE (1992) Relativistic, perpendicular shocks in electron-positron plasmas. ApJ 391:73-101. https://doi.org/10.1086/171326

Gargaté L, Bingham R, Fonseca RA, Silva LO (2007) dHybrid: a massively parallel code for hybrid simulations of space plasmas. Comput Phys Commun 176:419-425. https://doi.org/10.1016/j.cpc. 2006.11.013. arXiv:physics/0611174

Gary SP (1991) Electromagnetic ion/ion instabilities and their consequences in space plasmas—a review. Space Sci Rev 56:373-415. https://doi.org/10.1007/BF00196632

Gekelman W, Pfister H, Lucky Z, Bamber J, Leneman D, Maggs J (1991) Design, construction, and properties of the large plasma research device: the LAPD at UCLA. Rev Sci Instrum 62:2875-2883. https:// doi.org/10.1063/1.1142175

Gekelman W, Lawrence E, Collette A, Vincena S, Compernolle BV, Pribyl P, Berger M, Campbell J (2010) Magnetic field line reconnection in the current systems of flux ropes and alfvén waves. Phys Scripta T142:014032. https://doi.org/10.1088/0031-8949/2010/t142/014032

Gekelman W, Pribyl P, Lucky Z, Drandell M, Leneman D, Maggs J, Vincena S, Van Compernolle B, Tripathi SKP, Morales G, Carter TA, Wang Y, DeHaas T (2016) The upgraded large plasma device, a machine 
for studying frontier basic plasma physics. Rev Sci Instrum 87:025105. https://doi.org/10.1063/1. 4941079

Ghisellini G, Maraschi L, Treves A (1985) Inhomogeneous synchrotron-self-Compton models and the problem of relativistic beaming of BL Lac objects. A\&A 146:204-212

Ghizzo A, Bertrand P, Shoucri M, Johnston T, Fualkow E, Feix M (1990) A Vlasov code for the numerical simulation of stimulated raman scattering. J Comput Phys 90:431-457. https://doi.org/10.1016/00219991(90)90174-Y

Ghizzo A, Bertrand P, Johnston TW, Besse N (2009) Eulerian Vlasov models for plasma simulation: review and advances. Lecture note ISSS9: the 9th international school of space simulations

Gialis D, Pelletier G (2004) Which acceleration process for ultra high energy cosmic rays in gamma ray bursts? A\&A 425:395-403. https://doi.org/10.1051/0004-6361:20034301. arXiv:astro-ph/0402586

Giannios D (2010) UHECRs from magnetic reconnection in relativistic jets. MNRAS 408:L46-L50. https:// doi.org/10.1111/j.1745-3933.2010.00925.x. arXiv:1007.1522

Giannios D, Uzdensky DA, Begelman MC (2009) Fast TeV variability in blazars: jets in a jet. MNRAS 395:L29-L33. https://doi.org/10.1111/j.1745-3933.2009.00635.x. arXiv:0901.1877

Gieseler UDJ, Jones TW, Kang H (2000) Time dependent cosmic-ray shock acceleration with self-consistent injection. A\&A 364:911-922 arXiv:astro-ph/0011058

Girichidis P, Naab T, Walch S, Hanasz M (2014) Anisotropic transport and early dynamical impact of cosmic rays around supernova remnants. ArXiv e-prints arXiv: 1406.4861

Giroletti M, Giovannini G, Feretti L, Cotton WD, Edwards PG, Lara L, Marscher AP, Mattox JR, Piner BG, Venturi T (2004) Parsec-scale properties of markarian 501. ApJ 600:127-140. https://doi.org/10. 1086/379663. arXiv:astro-ph/0309285

Godfrey BB (1974) Numerical Cherenkov instabilities in electromagnetic particle codes. J Comput Phys 15:504-521. https://doi.org/10.1016/0021-9991(74)90076-X

Godfrey BB, Vay JL (2014) Suppressing the numerical Cherenkov instability in FDTD PIC codes. J Comput Phys 267:1-6. https://doi.org/10.1016/j.jcp.2014.02.022. arXiv:1401.0838

Godfrey BB, Vay JL (2015) Improved numerical Cherenkov instability suppression in the generalized PSTD PIC algorithm. Comput Phys Commun 196:221-225. https://doi.org/10.1016/j.cpc.2015.06. 008. arXiv: 1502.01387

Goetz JA, Dexter RN, Prager SC (1991) Total-magnetic reconnection during a major disruption in a tokamak. Phys Rev Lett 66:608-611. https://doi.org/10.1103/PhysRevLett.66.608

Gonoskov A, Marklund M (2018) Radiation-dominated particle and plasma dynamics. Phys Plasmas 25:093109. https://doi.org/10.1063/1.5047799. arXiv:1707.05749

Gonzalez-Herrero D, Boella E, Lapenta G (2018) Performance analysis and implementation details of the energy conserving semi-implicit method code (ECsim). Comput Phys Commun 229:162-169. https:// doi.org/10.1016/j.cpc.2018.03.020. arXiv:1711.05051

Gonzalez W, Parker E (eds) (2016) Magnetic reconnection. Astrophysics and Space Science Library, vol 427. Springer, Berlin, Heidelberg. https://doi.org/10.1007/978-3-319-26432-5

Grassi A (2017) Collisionless shocks in the context of Laboratory Astrophysics. PhD thesis, Universite Pierre et Marie Curie - Paris VI. https://tel.archives-ouvertes.fr/tel-01793040

Grassi A, Fedeli L, Macchi A, Bulanov SV, Pegoraro F (2014) Phase space dynamics after the breaking of a relativistic langmuir wave in a thermal plasma. Eur Phys J D 68(6):178. https://doi.org/10.1140/ epjd/e2014-50153-0

Grassi A, Fedeli L, Sgattoni A, Macchi A (2016) Vlasov simulation of laser-driven shock acceleration and ion turbulence. Plasma Phys Control Fusion 58:034021. https://doi.org/10.1088/0741-3335/58/ $3 / 034021$

Grassi A, Grech M, Amiranoff F, Macchi A, Riconda C (2017) Radiation-pressure-driven ion Weibel instability and collisionless shocks. Phys Rev E 96:033204. https://doi.org/10.1103/PhysRevE.96. 033204

Greenwood AD, Cartwright KL, Luginsland JW, Baca EA (2004) On the elimination of numerical Cerenkov radiation in PIC simulations. J Comput Phys 201:665-684. https://doi.org/10.1016/j.jcp.2004.06.021

Griebel M, Zumbusch G (1999) Parallel multigrid in an adaptive PDE solver based on hashing and spacefilling curves. Parallel Comput 25:827-843. https://doi.org/10.1016/S0167-8191(99)00020-4

Gruzinov A (2001) Gamma-ray burst phenomenology, shock dynamo, and the first magnetic fields. ApJ 563:L15-L18. https://doi.org/10.1086/324223. arXiv:astro-ph/0107106

Gruzinov A, Waxman E (1999) Gamma-ray burst afterglow: polarization and analytic light curves. ApJ 511:852-861. https://doi.org/10.1086/306720. arXiv:astro-ph/9807111 
Guo F, Giacalone J (2013) The acceleration of thermal protons at parallel collisionless shocks: three-dimensional hybrid simulations. ApJ 773:158. https://doi.org/10.1088/0004-637X/773/2/158. arXiv: 1303.5174

Guo F, Li H, Daughton W, Liu YH (2014a) Formation of hard power laws in the energetic particle spectra resulting from relativistic magnetic reconnection. Phys Rev Lett 113:155005. https://doi.org/10.1103/ PhysRevLett.113.155005. arXiv:1405.4040

Guo X, Sironi L, Narayan R (2014b) Non-thermal electron acceleration in low mach number collisionless shocks. I. Particle energy spectra and acceleration mechanism. ApJ 794:153. https://doi.org/10.1088/ 0004-637X/794/2/153. arXiv: 1406.5190

Guo X, Sironi L, Narayan R (2014c) Non-thermal electron acceleration in low mach number collisionless Shocks. II. Firehose-mediated Fermi acceleration and its dependence on pre-shock conditions. ApJ 797:47. https://doi.org/10.1088/0004-637X/797/1/47. arXiv:1409.7393

Guo F, Liu YH, Daughton W, Li H (2015) Particle acceleration and plasma dynamics during magnetic reconnection in the magnetically dominated regime. ApJ 806:167. https://doi.org/10.1088/0004-637X/806/ 2/167. arXiv:1504.02193

Guo F, Li X, Li H, Daughton W, Zhang B, Lloyd-Ronning N, Liu YH, Zhang H, Deng W (2016) Efficient production of high-energy nonthermal particles during magnetic reconnection in a magnetically dominated ion-electron plasma. ApJ 818:L9. https://doi.org/10.3847/2041-8205/818/1/L9. arXiv:1511.01434

Haberberger D, Ivancic S, Hu SX, Boni R, Barczys M, Craxton RS, Froula DH (2014) Measurements of electron density profiles using an angular filter refractometer. Phys Plasmas 21:056304. https://doi. org/10.1063/1.4873899

Hall DE, Sturrock PA (1967) Diffusion, scattering, and acceleration of particles by stochastic electromagnetic fields. Phys Fluids 10:2620-2628. https://doi.org/10.1063/1.1762084

Hanasz M, Lesch H (1993) Magnetic buoyancy and the galactic dynamo. A\&A 278:561-568

Hanasz M, Lesch H (2000) Cosmic-ray evolution in Parker-unstable galactic magnetic fields. ApJ 543:235244. https://doi.org/10.1086/317077

Hanasz M, Lesch H (2003) Incorporation of cosmic ray transport into the ZEUS MHD code. Application for studies of Parker instability in the ISM. A\&A 412:331-339. https://doi.org/10.1051/0004-6361: 20031433. arXiv:astro-ph/0309660

Hanasz M, Kowal G, Otmianowska-Mazur K, Lesch H (2004) Amplification of galactic magnetic fields by the cosmic-ray-driven dynamo. ApJ 605:L33-L36. https://doi.org/10.1086/420697. arXiv:astro-ph/0402662

Hanasz M, Wóltański D, Kowalik K (2009) Global galactic dynamo driven by cosmic rays and exploding magnetized stars. ApJ 706:L155-L159. https://doi.org/10.1088/0004-637X/706/1/L155. arXiv:0907.4891

Hanasz M, Kowalik K, Wóltański D, Pawłaszek R (2010) The PIERNIK MHD code—a multi-fluid, nonideal extension of the relaxing-TVD scheme (I). In: Goździewski K, Niedzielski A, Schneider J (eds) Extrasolar planets in multi-body systems: theory and observations, EDP sciences, EAS publications series, vol 42, pp 275-280. https://doi.org/10.1051/eas/1042029. arXiv:0812.2161

Hanusch A, Liseykina TV, Malkov M (2019) Acceleration of cosmic rays in supernova shocks: elemental selectivity of the injection mechanism. ApJ 872:108. https://doi.org/10.3847/1538-4357/aafdae. arXiv: 1803.00428

Hare JD, Suttle L, Lebedev SV, Loureiro NF, Ciardi A, Burdiak GC, Chittenden JP, Clayson T, Garcia C, Niasse N, Robinson T, Smith RA, Stuart N, Suzuki-Vidal F, Swadling GF, Ma J, Wu J, Yang Q (2017) Anomalous heating and plasmoid formation in a driven magnetic reconnection experiment. Phys Rev Lett 118:085001. https://doi.org/10.1103/PhysRevLett.118.085001

Harlow FH (2004) Fluid dynamics in Group T-3 Los Alamos National Laboratory: (LA-UR-03-3852). J Comput Phys 195:414-433. https://doi.org/10.1016/j.jcp.2003.09.031

Harlow FH, Amsden AA (1968) Numerical calculation of almost incompressible flow. J Comput Phys 3:80-93. https://doi.org/10.1016/0021-9991(68)90007-7

Harned DS, Schnack DD (1986) Semi-implicit method for long time scale magnetohydrodynamic computations in three dimensions. J Comput Phys 65:57-70. https://doi.org/10.1016/0021-9991(86)900045

Harten A, Lax PD, van Leer B (1983) On upstream differencing and Godunov-type schemes for hyperbolic conservation laws. SIAM Rev 25:35-61

Haugbølle T (2011) Three-dimensional modeling of relativistic collisionless Ion-electron Shocks. ApJ 739:L42. https://doi.org/10.1088/2041-8205/739/2/L42. arXiv:1007.5082 
Haugbølle T, Frederiksen JT, Nordlund $\AA$ (2013) photon-plasma: a modern high-order particle-in-cell code. Phys Plasmas 20:062904. https://doi.org/10.1063/1.4811384

Hededal CB, Haugbølle T, Trier Frederiksen J, Nordlund A (2004) Non-Fermi Power-Law Acceleration in Astrophysical Plasma Shocks. Astrophys J 617(2):L107-L110. https://doi.org/10.1086/427387

Hededal CB, Nishikawa KI (2005) The influence of an ambient magnetic field on relativistic collisionless plasma shocks. ApJ 623:L89-L92. https://doi.org/10.1086/430253. arXiv:astro-ph/0412317

Henri G, Pelletier G, Petrucci PO, Renaud N (1999) Active galactic nuclei as high energy engines. Astropart Phys 11:347-356. https://doi.org/10.1016/S0927-6505(98)00071-1. arXiv:astro-ph/9901051

Hesse M, Aunai N, Zenitani S, Kuznetsova M, Birn J (2013) Aspects of collisionless magnetic reconnection in asymmetric systems. Phys Plasmas 20:061210. https://doi.org/10.1063/1.4811467

Higashimori K, Hoshino M (2012) The relation between ion temperature anisotropy and formation of slow shocks in collisionless magnetic reconnection. J Geophys Res 117:A01220. https://doi.org/10.1029/ 2011JA016817. arXiv:1201.4213

Higuera AV, Cary JR (2017) Structure-preserving second-order integration of relativistic charged particle trajectories in electromagnetic fields. Phys Plasmas 24:052104. https://doi.org/10.1063/1.4979989. arXiv: 1701.05605

Hoshino M (2008) Wakefield acceleration by radiation pressure in relativistic shock waves. ApJ 672:940956. https://doi.org/10.1086/523665. arXiv:0709.1783

Hoshino M, Shimada N (2002) Nonthermal electrons at high mach number shocks: electron shock surfing acceleration. ApJ 572:880-887. https://doi.org/10.1086/340454. arXiv:astro-ph/0203073

Hoshino M, Arons J, Gallant YA, Langdon AB (1992) Relativistic magnetosonic shock waves in synchrotron sources—shock structure and nonthermal acceleration of positrons. ApJ 390:454-479. https://doi.org/ $10.1086 / 171296$

Howes GG (2018) Laboratory space physics: investigating the physics of space plasmas in the laboratory. Phys Plasmas 25:055501. https://doi.org/10.1063/1.5025421

Hudson PD (1965) Reflection of charged particles by plasma shocks. MNRAS 131:23. https://doi.org/10. 1093/mnras/131.1.23

Huntington CM, Fiuza F, Ross JS, Zylstra AB, Drake RP, Froula DH, Gregori G, Kugland NL, Kuranz CC, Levy MC, Li CK, Meinecke J, Morita T, Petrasso R, Plechaty C, Remington BA, Ryutov DD, Sakawa Y, Spitkovsky A, Takabe H, Park HS (2015) Observation of magnetic field generation via the Weibel instability in interpenetrating plasma flows. Nature Phys 11:173 EP. https://doi.org/10.1038/ nphys 3178

Inglebert A, Ghizzo A, Reveille T, Sarto DD, Bertrand P, Califano F (2011) A multi-stream Vlasov modeling unifying relativistic Weibel-type instabilities. EPL (Europhys Lett) 95:45002. https://doi.org/10.1209/ 0295-5075/95/45002

Innocenti ME, Lapenta G, Markidis S, Beck A, Vapirev A (2013) A multi level multi domain method for particle in cell plasma simulations. J Comput Phys 238:115-140. https://doi.org/10.1016/j.jcp.2012. 12.028. arXiv: 1201.6208

Inoue T (2019) Bell-instability-mediated spectral modulation of hadronic gamma-rays from a supernova remnant interacting with a molecular cloud. ApJ 872:46. https://doi.org/10.3847/1538-4357/aafb70. arXiv: 1902.04718

Ipavich FM (1975) Galactic winds driven by cosmic rays. ApJ 196:107-120. https://doi.org/10.1086/153397

Iwamoto M, Amano T, Hoshino M, Matsumoto Y (2017) Persistence of precursor waves in two-dimensional relativistic shocks. ApJ 840:52. https://doi.org/10.3847/1538-4357/aa6d6f. arXiv:1704.04411

Jafari A, Vishniac E (2018) Introduction to magnetic reconnection. ArXiv e-prints arXiv:1805.01347

Jaroschek CH, Hoshino M (2009) Radiation-dominated relativistic current sheets. Phys Rev Lett 103:075002. https://doi.org/10.1103/PhysRevLett.103.075002

Ji H, Daughton W (2011) Phase diagram for magnetic reconnection in heliophysical, astrophysical, and laboratory plasmas. Phys Plasmas 18:111207. https://doi.org/10.1063/1.3647505. arXiv:1109.0756

Ji H, Yamada M, Hsu S, Kulsrud R (1998) Experimental test of the sweet-parker model of magnetic reconnection. Phys Rev Lett 80:3256-3259. https://doi.org/10.1103/PhysRevLett.80.3256

Jiang YF, Oh SP (2018) A new numerical scheme for cosmic-ray transport. ApJ 854:5. https://doi.org/10. 3847/1538-4357/aaa6ce. arXiv:1712.07117

Jones FC, Ellison DC (1991) The plasma physics of shock acceleration. Space Sci Rev 58:259-346. https:// doi.org/10.1007/BF01206003 
Jones TW, Kang H (2005) An efficient numerical scheme for simulating particle acceleration in evolving cosmic-ray modified shocks. Astropart Phys 24:75-91. https://doi.org/10.1016/j.astropartphys.2005. 05.006. arXiv:astro-ph/0506212

Jones TW, Ryu D, Engel A (1999) Simulating electron transport and synchrotron emission in radio galaxies: shock acceleration and synchrotron aging in axisymmetric flows. ApJ 512:105-124. https://doi.org/ 10.1086/306772. arXiv:astro-ph/9809081

Jun BI, Clarke DA, Norman ML (1994) The evolution of cosmic-ray-mediated magnetohydrodynamic shocks: a two-fluid approach. ApJ 429:748-758. https://doi.org/10.1086/174358

Kagan D, Milosavljević M, Spitkovsky A (2013) A flux rope network and particle acceleration in threedimensional relativistic magnetic reconnection. ApJ 774:41. https://doi.org/10.1088/0004-637X/774/ 1/41. arXiv: 1208.0849

Kang H (2012) Diffusive shock acceleration with magnetic field amplification and Alfvenic drift. J Korean Astron Soc 45:127-138. https://doi.org/10.5303/JKAS.2012.45.5.127. arXiv:1209.5203

Kang H (2013) Effects of wave-particle interactions on diffusive shock acceleration at supernova remnants. J Korean Astron Soc 46:49-63. https://doi.org/10.5303/JKAS.2013.46.1.049

Kang H (2015) Particle acceleration in supernova remnants. Publ Korean Astron Soc 30:545-548. https:// doi.org/10.5303/PKAS.2015.30.2.545

Kang H, Jones TW (1991) Numerical studies of diffusive particle acceleration in supernova remnants. MNRAS 249:439-451. https://doi.org/10.1093/mnras/249.3.439

Kang H, Jones TW (2006) Numerical studies of diffusive shock acceleration at spherical shocks. Astropart Phys 25:246-258. https://doi.org/10.1016/j.astropartphys.2006.02.006. arXiv:astro-ph/0603223

Kang H, Jones TW (2007) Self-similar evolution of cosmic-ray-modified quasi-parallel plane shocks. Astropart Phys 28:232-246. https://doi.org/10.1016/j.astropartphys.2007.05.007. arXiv:0705.3274

Kang H, Jones TW, LeVeque RJ, Shyue KM (2001) Time evolution of cosmic-ray modified plane shocks. ApJ 550:737-751. https://doi.org/10.1086/319804. arXiv:astro-ph/0011538

Kang H, Ryu D, Jones TW (2009) Self-similar evolution of cosmic-ray modified shocks: the cosmic-ray spectrum. ApJ 695:1273-1288. https://doi.org/10.1088/0004-637X/695/2/1273. arXiv:0901.1702

Kang H, Edmon PP, Jones TW (2012) Nonthermal radiation from cosmic-ray modified shocks. ApJ 745:146. https://doi.org/10.1088/0004-637X/745/2/146

Kang H, Jones TW, Edmon PP (2013) Nonthermal radiation from supernova remnants: effects of magnetic field amplification and particle escape. ApJ 777:25. https://doi.org/10.1088/0004-637X/777/1/25. arXiv:1308.6652

Kato TN (2007) Relativistic collisionless shocks in unmagnetized electron-positron plasmas. ApJ 668:974979. https://doi.org/10.1086/521297. arXiv:0707.0545

Kato TN (2015) Particle acceleration and wave excitation in quasi-parallel high-mach-number collisionless shocks: particle-in-cell simulation. ApJ 802:115. https://doi.org/10.1088/0004-637X/802/2/115. arXiv:1407.1971

Katsouleas T, Dawson JM (1983) Unlimited electron acceleration in laser-driven plasma waves. Phys Rev Lett 51:392-395. https://doi.org/10.1103/PhysRevLett.51.392

Keppens R, Meliani Z, van Marle AJ, Delmont P, Vlasis A, van der Holst B (2012) Parallel, grid-adaptive approaches for relativistic hydro and magnetohydrodynamics. J Comput Phys 231:718-744. https:// doi.org/10.1016/j.jcp.2011.01.020

Keshet U, Waxman E (2005) Energy spectrum of particles accelerated in relativistic collisionless shocks. Phys Rev Lett 94:111102. https://doi.org/10.1103/PhysRevLett.94.111102. arXiv:astro-ph/0408489

Keshet U, Katz B, Spitkovsky A, Waxman E (2009) Magnetic field evolution in relativistic unmagnetized collisionless shocks. ApJ 693:L127-L130. https://doi.org/10.1088/0004-637X/693/2/L127. arXiv:0802.3217

Khiali B, de Gouveia Dal Pino EM, del Valle MV (2015) A magnetic reconnection model for explaining the multiwavelength emission of the microquasars Cyg X-1 and Cyg X-3. MNRAS 449:34-48. https:// doi.org/10.1093/mnras/stv248. arXiv:1406.5664

Kirk JG, Lyubarsky Y, Petri J (2009) The theory of pulsar winds and nebulae. In: Becker W (ed) Neutron stars and pulsars. Astrophysics and Space Science Library, vol 357. Springer, Berlin, Heidelberg, pp 421-450. https://doi.org/10.1007/978-3-540-76965-1_16. arXiv:astro-ph/0703116

Kirk JG (1994) Particle acceleration. In: Kirk JG, Melrose DB, Priest ER, Benz AO, Courvoisier TJL (eds) Plasma astrophysics. Saas-Fee Advanced Course, vol 24. Springer, Berlin, pp 225-314. https://doi. org/10.1007/3-540-31627-2_3 
Kirk JG (2004) Particle acceleration in relativistic current sheets. Phys Rev Lett 92:181101. https://doi.org/ 10.1103/PhysRevLett.92.181101. arXiv:astro-ph/0403516

Kirk JG, Dendy RO (2001) Shock acceleration of cosmic rays-a critical review. J Phys G: Nucl Phys 27:1589-1595. https://doi.org/10.1088/0954-3899/27/7/316. arXiv:astro-ph/0101175

Kirk JG, Skjæraasen O (2003) Dissipation in poynting-flux-dominated flows: the $\sigma$-problem of the Crab pulsar wind. ApJ 591:366-379. https://doi.org/10.1086/375215. arXiv:astro-ph/0303194

Kirk JG, Duffy P, Gallant YA (1996) Stochastic particle acceleration at shocks in the presence of braided magnetic fields. A\&A 314:1010-1016 arXiv:astro-ph/9604056

Kirk JG, Guthmann AW, Gallant YA, Achterberg A (2000) Particle acceleration at ultrarelativistic shocks: an eigenfunction method. ApJ 542:235-242. https://doi.org/10.1086/309533. arXiv:astro-ph/0005222

Kissmann R (2014) PICARD: a novel code for the galactic cosmic ray propagation problem. Astropart Phys 55:37-50. https://doi.org/10.1016/j.astropartphys.2014.02.002. arXiv:1401.4035

Klimas AJ (1987) A method for overcoming the velocity space filamentation problem in collisionless plasma model solutions. J Comput Phys 68:202-226. https://doi.org/10.1016/0021-9991(87)90052-0

Klimas A, Hesse M, Zenitani S, Kuznetsova M (2010) Particle-in-cell simulation of collisionless driven reconnection with open boundaries. Phys Plasmas 17:112904. https://doi.org/10.1063/1.3510480

Ko CM (1992) A note on the hydrodynamical description of cosmic ray propagation. A\&A 259:377-381

Koren B, van der Maarel HTM (1993) Monotone, higher-order accurate, multi-dimensional upwinding. In: Napolitano M, Sabetta F (eds) Numerical methods in fluid dynamics. Lecture Notes in Physics, vol 414. Springer, Berlin, pp 110-114. https://doi.org/10.1007/3-540-56394-6_198

Kosenko D, Ferrand G, Decourchelle A (2014) Parametric studies of cosmic ray acceleration in supernova remnants. MNRAS 443:1390-1401. https://doi.org/10.1093/mnras/stu1251. arXiv:1407.1818

Kowal G, Lazarian A, Vishniac ET, Otmianowska-Mazur K (2009) Numerical tests of fast reconnection in weakly stochastic magnetic fields. ApJ 700:63-85. https://doi.org/10.1088/0004-637X/700/1/63. arXiv:0903.2052

Kowal G, de Gouveia Dal Pino EM, Lazarian A (2011) Magnetohydrodynamic simulations of reconnection and particle acceleration: three-dimensional effects. ApJ 735:102. https://doi.org/10.1088/0004637X/735/2/102. arXiv:1103.2984

Kowal G, Lazarian A, Vishniac ET, Otmianowska-Mazur K (2012) Reconnection studies under different types of turbulence driving. Nonlinear Proc Geophys 19:297-314. https://doi.org/10.5194/npg-19297-2012. arXiv:1203.2971

Kowal G, Falceta-Gonçalves DA, Lazarian A, Vishniac ET (2017) Statistics of reconnection-driven turbulence. ApJ 838:91. https://doi.org/10.3847/1538-4357/aa6001. arXiv:1611.03914

Kronberg PP, Colgate SA, Li H, Dufton QW (2004) Giant radio galaxies and cosmic-ray acceleration. ApJ 604:L77-L80. https://doi.org/10.1086/383614

Kropotina YA, Bykov AM, Krasil'shchikov AM, Levenfish KP (2016) Relaxation of heavy ions in collisionless shock waves in cosmic plasma. J Technical Phys 61:517-524. https://doi.org/10.1134/ S1063784216040149

Kruells WM, Achterberg A (1994) Computation of cosmic-ray acceleration by Ito's stochastic differential equations. A\&A 286:314-327

Krymskii GF (1977) A regular mechanism for the acceleration of charged particles on the front of a shock wave. Doklady Akademiia Nauk SSSR 234:1306-1308

Kudoh Y, Hanawa T (2016) Approximate Riemann solvers for the cosmic ray magnetohydrodynamical equations. MNRAS 462:4517-4531. https://doi.org/10.1093/mnras/stw1937. arXiv:1608.03206

Kugland NL, Ryutov DD, Chang PY, Drake RP, Fiksel G, Froula DH, Glenzer SH, Gregori G, Grosskopf M, Koenig M, Kuramitsu Y, Kuranz C, Levy MC, Liang E, Meinecke J, Miniati F, Morita T, Pelka A, Plechaty C, Presura R, Ravasio A, Remington BA, Reville B, Ross JS, Sakawa Y, Spitkovsky A, Takabe H, Park HS (2012) Self-organized electromagnetic field structures in laser-produced counter-streaming plasmas. Nature Phys 8:809 EP. https://doi.org/10.1038/nphys2434

Kulsrud RM (1999) A critical review of galactic dynamos. ARA\&A 37:37-64. https://doi.org/10.1146/ annurev.astro.37.1.37

Kulsrud RM, Ferrari A (1971) The relativistic quasilinear theory of particle acceleration by hydromagnetic turbulence. Ap\&SS 12:302-318. https://doi.org/10.1007/BF00651420

Kulsrud R, Pearce WP (1969) The effect of wave-particle interactions on the propagation of cosmic rays. ApJ 156:445. https://doi.org/10.1086/149981 
Kunz MW, Stone JM, Bai XN (2014) Pegasus: a new hybrid-kinetic particle-in-cell code for astrophysical plasma dynamics. J Comput Phys 259:154-174. https://doi.org/10.1016/j.jcp.2013.11.035. arXiv:1311.4865

Kuramitsu Y, Sakawa Y, Morita T, Gregory CD, Waugh JN, Dono S, Aoki H, Tanji H, Koenig M, Woolsey N, Takabe H (2011) Time evolution of collisionless shock in counterstreaming laser-produced plasmas. Phys Rev Lett 106:175002. https://doi.org/10.1103/PhysRevLett.106.175002

Kuwabara T, Nakamura K, Ko CM (2004) Nonlinear parker instability with the effect of cosmic-ray diffusion. ApJ 607:828-839. https://doi.org/10.1086/383611. arXiv:astro-ph/0402350

Langdon AB, Birdsall CK (1970) Theory of plasma simulation using finite-size particles. Phys Fluids 13:2115-2122. https://doi.org/10.1063/1.1693209

Langdon AB, Arons J, Max CE (1988) Structure of relativistic magnetosonic shocks in electron-positron plasmas. Phys Rev Lett 61:779-782. https://doi.org/10.1103/PhysRevLett.61.779

Lapenta G (2008) Self-feeding turbulent magnetic reconnection on macroscopic scales. Phys Rev Lett 100:235001. https://doi.org/10.1103/PhysRevLett.100.235001. arXiv:0805.0426

Lapenta G, Lazarian A (2012) Achieving fast reconnection in resistive MHD models via turbulent means. Nonlinear Proc Geophys 19:251-263. https://doi.org/10.5194/npg-19-251-2012. arXiv:1110.0089

Lapenta G, Markidis S, Divin A, Newman D, Goldman M (2015) Separatrices: the crux of reconnection. J Plasma Phys 81:325810109. https://doi.org/10.1017/S0022377814000944. arXiv:1406.6141

Lapenta G, Ashour-Abdalla M, Walker RJ, El Alaoui M (2016) A multiscale study of ion heating in Earth's magnetotail. Geophys Res Lett 43:515-524. https://doi.org/10.1002/2015GL066689

Lapenta G, Gonzalez-Herrero D, Boella E (2017) Multiple-scale kinetic simulations with the energy conserving semi-implicit particle in cell method. J Plasma Phys 83:705830205. https://doi.org/10.1017/ S0022377817000137. arXiv:1612.08289

Lapeyre B, Pardoux E, Sentis R (2003) Introduction to Monte-Carlo methods for transport and diffusion equation. Oxford University Press, Oxford

Lautenbach S, Grauer R (2018) Multiphysics simulations of collisionless plasmas. Front Phys 6:113. https:// doi.org/10.3389/fphy.2018.00113. arXiv:1805.05698

Lazarian A, Opher M (2009) A model of acceleration of anomalous cosmic rays by reconnection in the heliosheath. ApJ 703:8-21. https://doi.org/10.1088/0004-637X/703/1/8. arXiv:0905.1120

Lazarian A, Vishniac ET (1999) Reconnection in a weakly stochastic field. ApJ 517:700-718. https://doi. org/10.1086/307233. arXiv:astro-ph/9811037

Lazarian A, Vlahos L, Kowal G, Yan H, Beresnyak A, de Gouveia Dal Pino EM (2012) Turbulence, magnetic reconnection in turbulent fluids and energetic particle acceleration. Space Sci Rev 173:557-622. https:// doi.org/10.1007/s11214-012-9936-7. arXiv:1211.0008

Lazarian A, Kowal G, Takamoto M, de Gouveia Dal Pino EM, Cho J (2016) Theory and applications of non-relativistic and relativistic turbulent reconnection. In: Gonzalez W, Parker E (eds) Magnetic reconnection: concepts and applications. Astrophysics and Space Science Library, vol 427. Springer, Cham, p 409. https://doi.org/10.1007/978-3-319-26432-5_11. arXiv:1512.03066

Lebiga O, Santos-Lima R, Yan H (2018) Kinetic-MHD simulations of gyroresonance instability driven by CR pressure anisotropy. MNRAS 476:2779-2791. https://doi.org/10.1093/mnras/sty309. arXiv: 1802.00746

Lee MA, Shapiro VD, Sagdeev RZ (1996) Pickup ion energization by shock surfing. J Geophys Res 101:4777-4790. https://doi.org/10.1029/95JA03570

Lee SH, Ellison DC, Nagataki S (2012) A generalized model of nonlinear diffusive shock acceleration coupled to an evolving supernova remnant. ApJ 750:156. https://doi.org/10.1088/0004-637X/750/2/ 156. arXiv: 1203.3614

Lefebvre E, Cochet N, Fritzler S, Malka V, Onard MMA, Chemin JF, Darbon S, Disdier L, Faure J, Fedotoff A, Landoas O, Malka G, Ot VM, Morel P, Gloahec MRL, Rouyer A, Rubbelynck C, Tikhonchuk V, Wrobel R, Audebert P, Rousseaux C (2003) Electron and photon production from relativistic laserplasma interactions. Nucl Fusion 43:629-633. https://doi.org/10.1088/0029-5515/43/7/317

Lehe R, Lifschitz A, Thaury C, Malka V, Davoine X (2013) Numerical growth of emittance in simulations of laser-wakefield acceleration. Phys Rev ST Accel Beams 16:021301. https://doi.org/10.1103/ PhysRevSTAB.16.021301

Lehe R, Kirchen M, Godfrey BB, Maier AR, Vay JL (2016) Elimination of numerical cherenkov instability in flowing-plasma particle-in-cell simulations by using galilean coordinates. Phys Rev E 94:053305. https://doi.org/10.1103/PhysRevE.94.053305 
Lembege B, Dawson JM (1987) Self-consistent study of a perpendicular collisionless and nonresistive shock. Phys Fluids 30:1767-1788. https://doi.org/10.1063/1.866191

Lembege B, Savoini P (1992) Nonstationarity of a two-dimensional quasiperpendicular supercritical collisionless shock by self-reformation. Phys Fluids B 4:3533-3548. https://doi.org/10.1063/1.860361

Lembège B, Savoini P, Hellinger P, Trávníček PM (2009) Nonstationarity of a two-dimensional perpendicular shock: competing mechanisms. J Geophys Res 114:A03217. https://doi.org/10.1029/ 2008JA013618

Lemoine M (2015) Nonlinear collisionless damping of Weibel turbulence in relativistic blast waves. J Plasma Phys 81:455810101. https://doi.org/10.1017/S0022377814000920. arXiv:1410.0146

Lemoine M, Pelletier G (2003) Particle transport in tangled magnetic fields and Fermi acceleration at relativistic shocks. ApJ 589:L73-L76. https://doi.org/10.1086/376353. arXiv:astro-ph/0304058

Lemoine M, Pelletier G (2010) On electromagnetic instabilities at ultra-relativistic shock waves. MNRAS 402:321-334. https://doi.org/10.1111/j.1365-2966.2009.15869.x. arXiv:0904.2657

Lemoine M, Pelletier G (2011) Dispersion and thermal effects on electromagnetic instabilities in the precursor of relativistic shocks. MNRAS 417:1148-1161. https://doi.org/10.1111/j.1365-2966.2011.19331. X. arXiv:1102.1308

Lemoine M, Revenu B (2006) Relativistic Fermi acceleration with shock compressed turbulence. MNRAS 366:635-644. https://doi.org/10.1111/j.1365-2966.2005.09912.x. arXiv:astro-ph/0510522

Lemoine M, Pelletier G, Revenu B (2006) On the efficiency of Fermi acceleration at relativistic shocks. ApJ 645:L129-L132. https://doi.org/10.1086/506322. arXiv:astro-ph/0606005

Lemoine M, Pelletier G, Gremillet L, Plotnikov I (2014) Current-driven filamentation upstream of magnetized relativistic collisionless shocks. MNRAS 440:1365-1378. https://doi.org/10.1093/mnras/stu213. arXiv: 1401.7166

Lemoine M, Gremillet L, Pelletier G, Vanthieghem A (2019a) Physics of Weibel-mediated relativistic collisionless shocks. Phys Rev Lett 123:035101. https://doi.org/10.1103/PhysRevLett.123.035101

Lemoine M, Pelletier G, Vanthieghem A, Gremillet L (2019b) Physics of relativistic collisionless shocks. III. The suprathermal particles. Phys Rev E 100(3):033210. https://doi.org/10.1103/PhysRevE.100. 033210

Lesch H, Hanasz M (2003) Strong magnetic fields and cosmic rays in very young galaxies. A\&A 401:809816. https://doi.org/10.1051/0004-6361:20030212. arXiv:astro-ph/0302162

Leveque RJ (1998) Nonlinear conservation laws and finite volume methods. In: Steiner O, Gautschy A (eds) Computational methods for astrophysical fluid flow. Saas-Fee Advanced Course, vol 27. Springer, Berlin, pp 1-159. https://doi.org/10.1007/3-540-31632-9_1

Li CK, Séguin FH, Frenje JA, Rygg JR, Petrasso RD, Town RPJ, Landen OL, Knauer JP, Smalyuk VA (2007) Observation of megagauss-field topology changes due to magnetic reconnection in laser-produced plasmas. Phys Rev Lett 99:055001. https://doi.org/10.1103/PhysRevLett.99.055001

Li G, Shalchi A, Ao X, Zank G, Verkhoglyadova OP (2012) Particle acceleration and transport at an oblique CME-driven shock. Adv Space Res 49:1067-1075. https://doi.org/10.1016/j.asr.2011.12.027

Li TC, Drake JF, Swisdak M (2014) Dynamics of double layers, ion acceleration, and heat flux suppression during solar flares. ApJ 793:7. https://doi.org/10.1088/0004-637X/793/1/7. arXiv:1408.1511

Li F, Yu P, Xu X, Fiuza F, Decyk VK, Dalichaouch T, Davidson A, Tableman A, An W, Tsung FS, Fonseca RA, Lu W, Mori WB (2017) Controlling the numerical Cerenkov instability in PIC simulations using a customized finite difference Maxwell solver and a local FFT based current correction. Comput Phys Commun 214:6-17. https://doi.org/10.1016/j.cpc.2017.01.001. arXiv:1605.01496

Lipatov AS (2002) The hybrid multiscale simulation technology: an introduction with application to astrophysical and laboratory plasmas. Springer, Berlin. https://doi.org/10.1007/978-3-662-05012-5

Liseikina TV, Pegoraro F, Echkina EY (2004) Foliation and mixing of the electron drift-kinetic distribution function in nonlinear two-dimensional magnetic reconnection. Phys Plasmas 11:3535-3545. https:// doi.org/10.1063/1.1758231

Liu QH (1997) The pstd algorithm: a time-domain method requiring only two cells per wavelength. Microwave Opt Technol Lett 15:158-165

Liu S, Petrosian V, Melia F (2004) Electron acceleration around the supermassive black hole at the Galactic Center. ApJ 611:L101-L104. https://doi.org/10.1086/423985. arXiv:astro-ph/0403487

Liu W, Li H, Yin L, Albright BJ, Bowers KJ, Liang EP (2011) Particle energization in 3D magnetic reconnection of relativistic pair plasmas. Phys Plasmas 18:052105-052105. https://doi.org/10.1063/ 1.3589304. arXiv: 1005.2435 
Liu YH, Drake JF, Swisdak M (2012) The structure of the magnetic reconnection exhaust boundary. Phys Plasmas 19:022110-022110. https://doi.org/10.1063/1.3685755. arXiv:1111.7039

Lobet M, Ruyer C, Debayle A, d'Humières E, Grech M, Lemoine M, Gremillet L (2015) Ultrafast synchrotron-enhanced thermalization of laser-driven colliding pair plasmas. Phys Rev Lett 115:215003. https://doi.org/10.1103/PhysRevLett.115.215003

Lobet M, d'Humières E, Grech M, Ruyer C, Davoine X, Gremillet L (2016) Modeling of radiative and quantum electrodynamics effects in pic simulations of ultra-relativistic laser-plasma interaction. $\mathrm{J}$ Phys: Conf Ser 688:012058

Lohner R (1987) An adaptive finite element scheme for transient problems in CFD. Comput Meth Appl Mech Eng 61:323-338. https://doi.org/10.1016/0045-7825(87)90098-3

Loureiro NF, Uzdensky DA (2016) Magnetic reconnection: from the Sweet-Parker model to stochastic plasmoid chains. Plasma Phys Control Fusion 58:014021. https://doi.org/10.1088/0741-3335/58/1/ 014021. arXiv:1507.07756

Loureiro NF, Schekochihin AA, Cowley SC (2007) Instability of current sheets and formation of plasmoid chains. Phys Plasmas 14:100703-100703. https://doi.org/10.1063/1.2783986. arXiv:astro-ph/0703631

Lucek SG, Bell AR (2000) Non-linear amplification of a magnetic field driven by cosmic ray streaming. MNRAS 314:65-74. https://doi.org/10.1046/j.1365-8711.2000.03363.x

Lyubarsky Y (2006) Electron-Ion coupling upstream of relativistic collisionless shocks. ApJ 652:12971305. https://doi.org/10.1086/508606. arXiv:astro-ph/0611015

Lyubarsky Y (2018) Interaction of the electromagnetic precursor from a relativistic shock with the upstream flow-I. Synchrotron absorption of strong electromagnetic waves. MNRAS 474:1135-1142. https:// doi.org/10.1093/mnras/stx2832. arXiv:1711.09581

Makwana KD, Keppens R, Lapenta G (2017) Two-way coupling of magnetohydrodynamic simulations with embedded particle-in-cell simulations. Comput Phys Commun 221:81-94. https://doi.org/10.1016/j. cpc.2017.08.003. arXiv:1708.02877

Malkov MA (1997a) Analytic solution for nonlinear shock acceleration in the Bohm limit. ApJ 485:638654. https://doi.org/10.1086/304471. arXiv:astro-ph/9707152

Malkov MA (1997b) Bifurcation, efficiency, and the role of injection in shock acceleration with the Bohm diffusion. ApJ 491:584-595. https://doi.org/10.1086/304990. arXiv:astro-ph/9708107

Malkov MA (1998) Ion leakage from quasiparallel collisionless shocks: implications for injection and shock dissipation. Phys Rev E 58:4911-4928. https://doi.org/10.1103/PhysRevE.58.4911. arXiv:astro-ph/9806340

Malkov MA, Drury LO (2001) Nonlinear theory of diffusive acceleration of particles by shock waves. Rep Prog Phys 64:429-481. https://doi.org/10.1088/0034-4885/64/4/201

Malkov MA, Voelk HJ (1995) Theory of ion injection at shocks. A\&A 300:605

Manfredi G, Shoucri M, Feix M, Bertrand P, Fijalkow E, Ghizzo A (1995) The numerical integration of the Vlasov equation possessing an invariant. J Comput Phys 121:298-313. https://doi.org/10.1016/ S0021-9991(95)90136-1

Mangeney A, Califano F, Cavazzoni C, Travnicek P (2002) A numerical scheme for the integration of the Vlasov-Maxwell system of equations. J Comput Phys 179:495-538. https://doi.org/10.1006/jcph. 2002.7071

Marcowith A, Casse F (2010) Postshock turbulence and diffusive shock acceleration in young supernova remnants. A\&A 515:A90. https://doi.org/10.1051/0004-6361/200913022. arXiv:1001.2111

Marcowith A, Kirk JG (1999) Computation of diffusive shock acceleration using stochastic differential equations. A\&A 347:391-400 arXiv:astro-ph/9905176

Marcowith A, Pelletier G, Henri G (1997) Acceleration mechanism in compact objects. A\&A 323:271-285

Marcowith A, Bret A, Bykov A, Dieckman ME, O'C Drury L, Lembège B, Lemoine M, Morlino G, Murphy G, Pelletier G, Plotnikov I, Reville B, Riquelme M, Sironi L, Stockem Novo A (2016) The microphysics of collisionless shock waves. Rep Prog Phys 79:046901. https://doi.org/10.1088/00344885/79/4/046901. arXiv:1604.00318

Marcowith A, Dwarkadas VV, Renaud M, Tatischeff V, Giacinti G (2018) Core-collapse supernovae as cosmic ray sources. MNRAS 479:4470-4485. https://doi.org/10.1093/mnras/sty1743. arXiv:1806.09700

Marcowith A, Belmont R, Malzac J (2013) Stochastic acceleration in accreting black holes. In: An INTEGRAL view of the high-energy sky (the first 10 years), Proceedings of Science. SISSA. https://doi. org/10.22323/1.176.0134. arXiv:1303.0712 
Mardahl P, Verboncoeur J (1997) Charge conservation in electromagnetic PIC codes; spectral comparison of Boris/DADI and Langdon-Marder methods. Comput Phys Commun 106:219-229. https://doi.org/ 10.1016/S0010-4655(97)00094-5

Markidis S, Lapenta G, Rizwan-uddin (2010) Multi-scale simulations of plasma with iPIC3D. Math Comput Simul 80:1509-1519. https://doi.org/10.1016/j.matcom.2009.08.038 multiscale modeling of moving interfaces in materials

Markidis S, Henri P, Lapenta G, Divin A, Goldman M, Newman D, Laure E (2013) Kinetic simulations of plasmoid chain dynamics. Phys Plasmas 20:082105. https://doi.org/10.1063/1.4817286. arXiv:1306.1050

Markidis S, Henri P, Lapenta G, Rönnmark K, Hamrin M, Meliani Z, Laure E (2014) The fluid-kinetic particle-in-cell method for plasma simulations. J Comput Phys 271:415-429. https://doi.org/10.1016/ j.jcp.2014.02.002. arXiv:1306.1089

Martí JM, Müller E (2015) Grid-based methods in relativistic hydrodynamics and magnetohydrodynamics. Living Rev Comput Astrophys 1:3. https://doi.org/10.1007/lrca-2015-3

Martinez B (2018) Radiative and quantum electrodynamic effects in ultra-relativistic laser-matter interaction. PhD thesis, University of Bordeaux

Martins SF, Fonseca RA, Silva LO, Mori WB (2009) Ion dynamics and acceleration in relativistic shocks. ApJ 695:L189-L193. https://doi.org/10.1088/0004-637X/695/2/L189. arXiv:0903.3573

Masters A, Sulaiman AH, Sergis N, Stawarz L, Fujimoto M, Coates AJ, Dougherty MK (2016) Suprathermal electrons at Saturn's bow shock. ApJ 826:48. https://doi.org/10.3847/0004-637X/826/1/48. arXiv: 1606.01685

Matsukiyo S, Scholer M (2003) Modified two-stream instability in the foot of high Mach number quasiperpendicular shocks. J Geophys Res 108:1459. https://doi.org/10.1029/2003JA010080

Matsumoto Y, Amano T, Hoshino M (2012) Electron accelerations at high Mach number shocks: twodimensional particle-in-cell simulations in various parameter regimes. ApJ 755:109. https://doi.org/ 10.1088/0004-637X/755/2/109. arXiv:1204.6312

Matsumoto Y, Amano T, Kato TN, Hoshino M (2017) Electron surfing and drift accelerations in a Weibeldominated high-Mach-number shock. ArXiv e-prints arXiv:1709.03673

Mauk BH, Haggerty DK, Paranicas C, Clark G, Kollmann P, Rymer AM, Mitchell DG, Bolton SJ, Levin SM, Adriani A, Allegrini F, Bagenal F, Connerney JEP, Gladstone GR, Kurth WS, McComas DJ, Ranquist D, Szalay JR, Valek P (2017) Juno observations of energetic charged particles over Jupiter's polar regions: analysis of monodirectional and bidirectional electron beams. Geophys Res Lett 44:44104418. https://doi.org/10.1002/2016GL072286

McKinney JC, Uzdensky DA (2012) A reconnection switch to trigger gamma-ray burst jet dissipation. MNRAS 419:573-607. https://doi.org/10.1111/j.1365-2966.2011.19721.x. arXiv:1011.1904

Medvedev MV, Loeb A (1999) Generation of magnetic fields in the relativistic shock of gamma-ray burst sources. ApJ 526:697-706. https://doi.org/10.1086/308038. arXiv:astro-ph/9904363

Melrose DB (1980) Plasma astrophysics. Nonthermal processes in diffuse magnetized plasmas, vol 1: the emission, absorption and transfer of waves in plasmas, vol 2: astrophysical applications. Gordon and Breach, New York

Melrose DB (1996) Particle acceleration and nonthermal radiation in space plasmas. Ap\&SS 242:209-246. https://doi.org/10.1007/BF00645114

Melzani M (2014) Collisionless magnetic reconnection in relativistic plasmas with particle-in-cell simulations. PhD thesis, Ecole normale supérieure de Lyon - ENS LYON. https://tel.archives-ouvertes.fr/ tel-01126912

Melzani M, Winisdoerffer C, Walder R, Folini D, Favre JM, Krastanov S, Messmer P (2013) Apar-T: code, validation, and physical interpretation of particle-in-cell results. A\&A 558:A133. https://doi.org/10. 1051/0004-6361/201321557. arXiv:1308.5892

Melzani M, Walder R, Folini D, Winisdoerffer C, Favre JM (2014a) Relativistic magnetic reconnection in collisionless ion-electron plasmas explored with particle-in-cell simulations. A\&A 570:A111. https:// doi.org/10.1051/0004-6361/201424083. arXiv:1404.7366

Melzani M, Walder R, Folini D, Winisdoerffer C, Favre JM (2014b) The energetics of relativistic magnetic reconnection: ion-electron repartition and particle distribution hardness. A\&A 570:A112. https://doi. org/10.1051/0004-6361/201424193. arXiv:1405.2938

Merloni A, Fabian AC (2001) Accretion disc coronae as magnetic reservoirs. MNRAS 321:549-552. https:// doi.org/10.1046/j.1365-8711.2001.04060.x. arXiv:astro-ph/0009498 
Meyer M, Horns D, Zechlin HS (2010) The Crab Nebula as a standard candle in very high-energy astrophysics. A\&A 523:A2. https://doi.org/10.1051/0004-6361/201014108. arXiv:1008.4524

Micono M, Zurlo N, Massaglia S, Ferrari A, Melrose DB (1999) Diffusive shock acceleration in extragalactic jets. A\&A 349:323-333 arXiv:astro-ph/9906440

Mignone A, Bodo G, Massaglia S, Matsakos T, Tesileanu O, Zanni C, Ferrari A (2007) PLUTO: a numerical code for computational astrophysics. ApJS 170:228-242. https://doi.org/10.1086/513316. arXiv:astro-ph/0701854

Mignone A, Bodo G, Vaidya B, Mattia G (2018) A particle module for the PLUTO code. I. An implementation of the MHD-PIC equations. ApJ 859:13. https://doi.org/10.3847/1538-4357/aabccd. arXiv: 1804.01946

Miller JA, Larosa TN, Moore RL (1996) Stochastic electron acceleration by cascading fast mode waves in impulsive solar flares. ApJ 461:445. https://doi.org/10.1086/177072

Milosavljević M, Nakar E (2006) The cosmic-ray precursor of relativistic collisionless shocks: a missing link in gamma-ray burst afterglows. ApJ 651:979-984. https://doi.org/10.1086/507975. arXiv:astro-ph/0512548

Miniati F (2001) COSMOCR: a numerical code for cosmic ray studies in computational cosmology. Comput Phys Commun 141:17-38. https://doi.org/10.1016/S0010-4655(01)00293-4. arXiv:astro-ph/0105447

Miniati F (2007) Glimm Godunov's method for cosmic-ray-hydrodynamics. J Comput Phys 227:776-796. https://doi.org/10.1016/j.jcp.2007.08.013. arXiv:astro-ph/0611499

Miyoshi T, Kusano K (2005) A multi-state HLL approximate Riemann solver for ideal magnetohydrodynamics. J Comput Phys 208:315-344. https://doi.org/10.1016/j.jcp.2005.02.017

Monaghan JJ (1992) Smoothed particle hydrodynamics. ARA\&A 30:543-574. https://doi.org/10.1146/ annurev.aa.30.090192.002551

Montag P, Egedal J, Lichko E, Wetherton B (2017) Impact of compressibility and a guide field on Fermi acceleration during magnetic island coalescence. Phys Plasmas 24:062906. https://doi.org/10.1063/ 1.4985302

Morita T, Sakawa Y, Tomita K, Ide T, Kuramitsu Y, Nishio K, Nakayama K, Inoue K, Moritaka T, Ide H, Kuwada M, Tsubouchi K, Uchino K, Takabe H (2013) Thomson scattering measurement of a shock in laser-produced counter-streaming plasmas. Phys Plasmas 20:092115. https://doi.org/10.1063/1. 4821967

Morlino G (2011) The role of ionization in the shock acceleration theory. MNRAS 412:2333-2344. https:// doi.org/10.1111/j.1365-2966.2010.18054.x. arXiv:1011.5180

Morlino G, Blasi P, Bandiera R, Amato E (2013) Broad Balmer line emission and cosmic ray acceleration efficiency in supernova remnant shocks. A\&A 558:A25. https://doi.org/10.1051/0004-6361/ 201322006. arXiv:1306.6454

Nam CH, Sung JH, Lee HW, Youn JW, Lee SK (2018) Performance of the 20 fs, 4 PW Ti: Sapphire Laser at CoReLS. In: Conference on lasers and electro-optics. Optical Society of America, p STu4O.3. https:// doi.org/10.1364/CLEO_SI.2018.STu4O.3

Nanbu K (1997) Theory of cumulative small-angle collisions in plasmas. Phys Rev E 55:4642-4652. https:// doi.org/10.1103/PhysRevE.55.4642

Nanbu K, Yonemura S (1998) Weighted particles in coulomb collision simulations based on the theory of a cumulative scattering angle. J Comput Phys 145:639-654. https://doi.org/10.1006/jcph.1998.6049

Nayakshin S, Melia F (1998) Self-consistent Fokker-Planck treatment of particle distributions in astrophysical plasmas. ApJS 114:269-288. https://doi.org/10.1086/313069. arXiv:astro-ph/9705011

Niel F, Riconda C, Amiranoff F, Duclous R, Grech M (2018) From quantum to classical modeling of radiation reaction: a focus on stochasticity effects. Phys Rev E 97:043209. https://doi.org/10.1103/ PhysRevE.97.043209

Niemann C, Gekelman W, Constantin CG, Everson ET, Schaeffer DB, Bondarenko AS, Clark SE, Winske D, Vincena S, Van Compernolle B, Pribyl P (2014) Observation of collisionless shocks in a large current-free laboratory plasma. Geophys Res Lett 41:7413. https://doi.org/10.1002/2014GL061820

Nilson PM, Willingale L, Kaluza MC, Kamperidis C, Minardi S, Wei MS, Fernandes P, Notley M, Bandyopadhyay S, Sherlock M, Kingham RJ, Tatarakis M, Najmudin Z, Rozmus W, Evans RG, Haines MG, Dangor AE, Krushelnick K (2006) Magnetic reconnection and plasma dynamics in two-beam laser-solid interactions. Phys Rev Lett 97:255001. https://doi.org/10.1103/PhysRevLett.97.255001

Nilson PM, Willingale L, Kaluza MC, Kamperidis C, Minardi S, Wei MS, Fernandes P, Notley M, Bandyopadhyay S, Sherlock M, Kingham RJ, Tatarakis M, Najmudin Z, Rozmus W, Evans RG, Haines MG, 
Dangor AE, Krushelnick K (2008) Bidirectional jet formation during driven magnetic reconnection in two-beam laser-plasma interactions. Phys Plasmas 15:092701. https://doi.org/10.1063/1.2966115

Nishikawa KI, Hardee P, Richardson G, Preece R, Sol H, Fishman GJ (2003) Particle acceleration in relativistic jets due to Weibel instability. ApJ 595:555-563. https://doi.org/10.1086/377260. arXiv:astro-ph/0305091

Noble SC, Gammie CF, McKinney JC, Del Zanna L (2006) Primitive variable solvers for conservative general relativistic magnetohydrodynamics. ApJ 641:626-637. https://doi.org/10.1086/500349. arXiv:astro-ph/0512420

Nuter R, Tikhonchuk V (2016) Suppressing the numerical cherenkov radiation in the yee numerical scheme. J Comput Phys 305:664-676. https://doi.org/10.1016/j.jcp.2015.10.057

Nuter R, Gremillet L, Lefebvre E, Lévy A, Ceccotti T, Martin P (2011) Field ionization model implemented in particle in cell code and applied to laser-accelerated carbon ions. Phys Plasmas 18:033107. https:// doi.org/10.1063/1.3559494

Nuter R, Grech M, de Alaiza Gonzalez, Martinez P, Bonnaud G, d'Humières E (2014) Maxwell solvers for the simulations of the laser-matter interaction. Eur Phys J D 68:177. https://doi.org/10.1140/epjd/ e2014-50162-y

Ohira Y (2016) Injection to rapid diffusive shock acceleration at perpendicular shocks in partially ionized plasmas. ApJ 827:36. https://doi.org/10.3847/0004-637X/827/1/36. arXiv:1606.03148

Ohtani H, Horiuchi R (2009) Open boundary condition for particle simulation in magnetic reconnection research. Plasma Fusion Res 4:024. https://doi.org/10.1585/pfr.4.024

Oishi JS, Mac Low MM, Collins DC, Tamura M (2015) Self-generated turbulence in magnetic reconnection. ApJ 806:L12. https://doi.org/10.1088/2041-8205/806/1/L12. arXiv:1505.04653

Olshevsky V, Lapenta G, Markidis S (2013) Energetics of kinetic reconnection in a three-dimensional null-point cluster. Phys Rev Lett 111:045002. https://doi.org/10.1103/PhysRevLett.111.045002

Olshevsky V, Bacchini F, Poedts S, Lapenta G (2019) Slurm: fluid particle-in-cell code for plasma modeling. Comput Phys Commun 235:16-24. https://doi.org/10.1016/j.cpc.2018.06.014

Orszag SA, Tang CM (1979) Small-scale structure of two-dimensional magnetohydrodynamic turbulence. J Fluid Mech 90:129-143. https://doi.org/10.1017/S002211207900210X

O'Sullivan S, Downes TP (2007) A three-dimensional numerical method for modelling weakly ionized plasmas. MNRAS 376:1648-1658. https://doi.org/10.1111/j.1365-2966.2007.11429.x. arXiv:astro-ph/0612580

Pakmor R, Pfrommer C, Simpson CM, Springel V (2016) Galactic winds driven by isotropic and anisotropic cosmic-ray diffusion in disk galaxies. ApJ 824:L30. https://doi.org/10.3847/2041-8205/824/2/L30. arXiv: 1605.00643

Palmroth M, Ganse U, Pfau-Kempf Y, Battarbee M, Turc L, Brito T, Grandin M, Hoilijoki S, Sandroos A, von Alfthan S (2018) Vlasov methods in space physics and astrophysics. Living Rev Comput Astrophys 4:1. https://doi.org/10.1007/s41115-018-0003-2. arXiv:1808.05885

Papini E, Landi S, Del Zanna L (2018) Fast magnetic reconnection: the ideal tearing instability in classic, Hall, and relativistic plasmas. J Phys: Conf Ser 1031:012020. https://doi.org/10.1088/1742-6596/ 1031/1/012020. arXiv:1801.10534

Parizot E (2004) GZK horizon and magnetic fields. Nucl Phys B: Proc Suppl 136:169-178. https://doi.org/ 10.1016/j.nuclphysbps.2004.10.034. arXiv:astro-ph/0409191

Parizot E, Marcowith A, Ballet J, Gallant YA (2006) Observational constraints on energetic particle diffusion in young supernovae remnants: amplified magnetic field and maximum energy. A\&A 453:387-395. https://doi.org/10.1051/0004-6361:20064985. arXiv:astro-ph/0603723

Park BT, Petrosian V (1996) Fokker-Planck equations of stochastic acceleration: a study of numerical methods. ApJS 103:255. https://doi.org/10.1086/192278

Park HS, Ryutov DD, Ross JS, Kugland NL, Glenzer SH, Plechaty C, Pollaine SM, Remington BA, Spitkovsky A, Gargate L, Gregori G, Bell A, Murphy C, Sakawa Y, Kuramitsu Y, Morita T, Takabe H, Froula DH, Fiksel G, Miniati F, Koenig M, Ravasio A, Pelka A, Liang E, Woolsey N, Kuranz CC, Drake RP, Grosskopf MJ (2012) Studying astrophysical collisionless shocks with counterstreaming plasmas from high power lasers. High Energy Density Phys 8:38-45 10.1016/j.hedp.2011.11.001

Park J, Caprioli D, Spitkovsky A (2015) Simultaneous acceleration of protons and electrons at nonrelativistic quasiparallel collisionless shocks. Phys Rev Lett 114:085003. https://doi.org/10.1103/PhysRevLett. 114.085003. arXiv:1412.0672

Parker EN (1955) Hydromagnetic waves and the acceleration of cosmic rays. Phys Rev 99:241-253. https:// doi.org/10.1103/PhysRev.99.241 
Parker EN (1957) Sweet's mechanism for merging magnetic fields in conducting fluids. J Geophys Res 62:509-520. https://doi.org/10.1029/JZ062i004p00509

Parker EN (1958a) Origin and dynamics of cosmic rays. Phys Rev 109:1328-1344. https://doi.org/10.1103/ PhysRev.109.1328

Parker EN (1958b) Suprathermal particle generation in the solar corona. ApJ 128:677. https://doi.org/10. $1086 / 146580$

Parker EN (1992) Fast dynamos, cosmic rays, and the Galactic magnetic field. ApJ 401:137-145. https:// doi.org/10.1086/172046

Patrignani C, Particle Data Group (2016) Review of particle physics. Chin Phys C 40:100001. https://doi. org/10.1088/1674-1137/40/10/100001

Pei W, Horiuchi R, Sato T (2001) Long time scale evolution of collisionless driven reconnection in a two-dimensional open system. Phys Plasmas 8:3251-3257. https://doi.org/10.1063/1.1375150

Pelletier G, Marcowith A (1998) Nonlinear dynamics in the relativistic plasma of astrophysical high-energy sources. ApJ 502:598-613. https://doi.org/10.1086/305914

Pelletier G, Lemoine M, Marcowith A (2009) On Fermi acceleration and magnetohydrodynamic instabilities at ultra-relativistic magnetized shock waves. MNRAS 393:587-597. https://doi.org/10.1111/j.13652966.2008.14219.x. arXiv:0807.3459

Pérez F, Gremillet L, Decoster A, Drouin M, Lefebvre E (2012) Improved modeling of relativistic collisions and collisional ionization in particle-in-cell codes. Phys Plasmas 19:083104. https://doi.org/10.1063/ 1.4742167

Pétri J, Lyubarsky Y (2007) Magnetic reconnection at the termination shock in a striped pulsar wind. A\&A 473:683-700. https://doi.org/10.1051/0004-6361:20066981

Petrosian V (2012) Stochastic acceleration by turbulence. Space Sci Rev 173:535-556. https://doi.org/10. 1007/s11214-012-9900-6. arXiv:1205.2136

Petschek HE (1964) Magnetic Field Annihilation. In: Hess WN (ed) The physics of solar flares. NASA special publication, vol 50. NASA, Washington, pp 425-439

Pfrommer C, Springel V, Enßlin TA, Jubelgas M (2006) Detecting shock waves in cosmological smoothed particle hydrodynamics simulations. MNRAS 367:113-131. https://doi.org/10.1111/j.1365-2966. 2005.09953.x. arXiv:astro-ph/0603483

Pfrommer C, Pakmor R, Schaal K, Simpson CM, Springel V (2017) Simulating cosmic ray physics on a moving mesh. MNRAS 465:4500-4529. https://doi.org/10.1093/mnras/stw2941. arXiv:1604.07399

Plotnikov I, Sironi L (2019) The synchrotron maser emission from relativistic shocks in Fast Radio Bursts: 1D PIC simulations of cold pair plasmas. MNRAS 485:3816-3833. https://doi.org/10.1093/mnras/ stz640. arXiv:1901.01029

Plotnikov I, Pelletier G, Lemoine M (2011) Particle transport in intense small-scale magnetic turbulence with a mean field. A\&A 532:A68. https://doi.org/10.1051/0004-6361/201117182. arXiv:1105.0618

Plotnikov I, Pelletier G, Lemoine M (2013) Particle transport and heating in the microturbulent precursor of relativistic shocks. MNRAS 430:1280-1293. https://doi.org/10.1093/mnras/sts696. arXiv:1206.6634

Plotnikov I, Grassi A, Grech M (2018) Perpendicular relativistic shocks in magnetized pair plasma. MNRAS 477:5238-5260. https://doi.org/10.1093/mnras/sty979. arXiv:1712.02883

Pontin DI (2011) Three-dimensional magnetic reconnection regimes: a review. Adv Space Res 47:15081522. https://doi.org/10.1016/j.asr.2010.12.022. arXiv:1101.0924

Powell KG, Roe PL, Linde TJ, Gombosi TI, De Zeeuw DL (1999) A solution-adaptive upwind scheme for ideal magnetohydrodynamics. J Comput Phys 154:284-309. https://doi.org/10.1006/jcph.1999.6299

Press WH, Teukolsky SA, Vetterling WT, Flannery BP (2002) Numerical recipes in C++: the art of scientific computing. Cambridge University Press, Cambridge

Priest ER (1994) Magnetohydrodynamics. In: Kirk JG, Melrose DB, Priest ER, Benz AO, Courvoisier TJL (eds) Plasma astrophysics. Saas-Fee Advanced Course, vol 24. Springer, Berlin, pp 1-112. https://doi. org/10.1007/3-540-31627-2_1

Pritchett PL (2005) The "Newton Challenge": kinetic aspects of forced magnetic reconnection. J Geophys Res 110(A9):A10213. https://doi.org/10.1029/2005JA011228

Pritchett PL (2013) The influence of intense electric fields on three-dimensional asymmetric magnetic reconnection. Phys Plasmas 20:061204. https://doi.org/10.1063/1.4811123

Provornikova E, Laming JM, Lukin VS (2016) Plasma compression in magnetic reconnection regions in the solar corona. ApJ 825:55. https://doi.org/10.3847/0004-637X/825/1/55. arXiv:1604.07325

Provornikova E, Laming JM, Lukin VS (2018) Reflection of fast magnetosonic waves near a magnetic reconnection region. ApJ 860:138. https://doi.org/10.3847/1538-4357/aac1c1. arXiv:1803.11212 
Pryadko JM, Petrosian V (1998) Stochastic acceleration of low-energy electrons in plasmas with finite temperature. ApJ 495:377-384. https://doi.org/10.1086/305271. arXiv:astro-ph/9706050

Ptuskin VS, Moskalenko IV, Jones FC, Strong AW, Zirakashvili VN (2006) Dissipation of magnetohydrodynamic waves on energetic particles: impact on interstellar turbulence and cosmic-ray transport. ApJ 642:902-916. https://doi.org/10.1086/501117. arXiv:astro-ph/0510335

Ptuskin V, Zirakashvili V, Seo ES (2010) Spectrum of galactic cosmic rays accelerated in supernova remnants. ApJ 718:31-36. https://doi.org/10.1088/0004-637X/718/1/31. arXiv:1006.0034

Pukhov A (2019) X-dispersionless Maxwell solver for plasma-based particle acceleration. arXiv: 1906.10500

Rasera Y, Chandran B (2008) Numerical simulations of buoyancy instabilities in galaxy cluster plasmas with cosmic rays and anisotropic thermal conduction. ApJ 685:105-117. https://doi.org/10.1086/591012. arXiv:0809.0354

Raymond AE, Dong CF, McKelvey A, Zulick C, Alexander N, Bhattacharjee A, Campbell PT, Chen H, Chvykov V, Del Rio E, Fitzsimmons P, Fox W, Hou B, Maksimchuk A, Mileham C, Nees J, Nilson PM, Stoeckl C, Thomas AGR, Wei MS, Yanovsky V, Krushelnick K, Willingale L (2018) Relativisticelectron-driven magnetic reconnection in the laboratory. Phys Rev E 98:043207. https://doi.org/10. 1103/PhysRevE.98.043207

Reis RC, Miller JM (2013) On the size and location of the X-ray emitting coronae around black holes. ApJ 769:L7. https://doi.org/10.1088/2041-8205/769/1/L7. arXiv:1304.4947

Remington BA, Arnett D, Drake RP, Takabe H (1999) Modeling astrophysical phenomena in the laboratory with intense lasers. Science 284:1488-1493. https://doi.org/10.1126/science.284.5419.1488

Remington BA, Drake RP, Ryutov DD (2006) Experimental astrophysics with high power lasers and $z$ pinches. Rev Mod Phys 78:755-807. https://doi.org/10.1103/RevModPhys.78.755

Reville B, Bell AR (2012) A filamentation instability for streaming cosmic rays. MNRAS 419:2433-2440. https://doi.org/10.1111/j.1365-2966.2011.19892.x. arXiv:1109.5690

Reville B, Bell AR (2013) Universal behaviour of shock precursors in the presence of efficient cosmic ray acceleration. MNRAS 430:2873-2884. https://doi.org/10.1093/mnras/stt100. arXiv:1301.3173

Reville B, Bell AR (2014) On the maximum energy of shock-accelerated cosmic rays at ultra-relativistic shocks. MNRAS 439:2050-2059. https://doi.org/10.1093/mnras/stu088. arXiv:1401.2803

Reville B, Kirk JG, Duffy P (2006) A current-driven instability in parallel, relativistic shocks. Plasma Phys Control Fusion 48:1741-1747. https://doi.org/10.1088/0741-3335/48/12/004. arXiv:astro-ph/0608462

Reynolds SP (1998) Models of synchrotron X-rays from shell supernova remnants. ApJ 493(1):375-396. https://doi.org/10.1086/305103

Reynolds DR, Samtaney R, Woodward CS (2006) A fully implicit numerical method for single-fluid resistive magnetohydrodynamics. J Comput Physics 219:144-162

Reynolds SP, Gaensler BM, Bocchino F (2012) Magnetic fields in supernova remnants and pulsar-wind nebulae. Space Sci Rev 166:231-261. https://doi.org/10.1007/s11214-011-9775-y. arXiv:1104.4047

Rieger FM, Duffy P (2006) A microscopic analysis of shear acceleration. ApJ 652:1044-1049. https://doi. org/10.1086/508056. arXiv:astro-ph/0610187

Rieke M, Trost T, Grauer R (2015) Coupled Vlasov and two-fluid codes on GPUs. J Comput Phys 283:436452. https://doi.org/10.1016/j.jcp.2014.12.016. arXiv:1406.5445

Ripin BH, Manka CK, Peyser TA, McLean EA, Stamper JA, Mostovych AN, Grun J, Kearney K, Crawford JR, Huba JD et al (1990) Laboratory laser-produced astrophysical-like plasmas. Laser Part Beams 8(1-2):183-190. https://doi.org/10.1017/S026303460000793X

Riquelme MA, Spitkovsky A (2011) Electron injection by Whistler waves in non-relativistic shocks. ApJ 733:63. https://doi.org/10.1088/0004-637X/733/1/63. arXiv:1009.3319

Risken H (1989) The Fokker-Planck equation: methods of solution and applications. Springer series in synergetics, 2nd edn. Springer, Berlin. https://doi.org/10.1007/978-3-642-61544-3

Roe PL (1981) Approximate Riemann solvers, parameter vectors, and difference schemes. J Comput Phys 43:357-372. https://doi.org/10.1016/0021-9991(81)90128-5

Romagnani L, Bulanov SV, Borghesi M, Audebert P, Gauthier JC, Löwenbrück K, Mackinnon AJ, Patel P, Pretzler G, Toncian T, Willi O (2008) Observation of collisionless shocks in laser-plasma experiments. Phys Rev Lett 101:025004. https://doi.org/10.1103/PhysRevLett.101.025004

Romanova MM, Lovelace RVE (1992) Magnetic field, reconnection, and particle acceleration in extragalactic jets. A\&A 262:26-36 
Romero GE, Vieyro FL, Chaty S (2014) Coronal origin of the polarization of the high-energy emission of Cygnus X-1. A\&A 562:L7. https://doi.org/10.1051/0004-6361/201323316. arXiv:1402.0524

Rose SJ (1994) Astrophysical plasma laboratories. Phys World 7:56-61. https://doi.org/10.1088/20587058/7/4/39

Rosenberg MJ, Li CK, Fox W, Igumenshchev I, Séguin FH, Town RPJ, Frenje JA, Stoeckl C, Glebov V, Petrasso RD (2015a) A laboratory study of asymmetric magnetic reconnection in strongly driven plasmas. Nature Commun 6:6190 EP. https://doi.org/10.1038/ncomms7190

Rosenberg MJ, Li CK, Fox W, Zylstra AB, Stoeckl C, Séguin FH, Frenje JA, Petrasso RD (2015b) Slowing of magnetic reconnection concurrent with weakening plasma inflows and increasing collisionality in strongly driven laser-plasma experiments. Phys Rev Lett 114:205004. https://doi.org/10.1103/ PhysRevLett.114.205004

Ross IN, White MS, E BJ, Craddock D, Damerell AR, Day JR, Gibson AF, Gottfeldt P, Nicholas DJ, Reason CJ (1981) Vulcan - a versatile high-power glass laser for multiuser experiments. IEEE J Quantum Electron QE-17:1653

Ross JS, Higginson DP, Ryutov D, Fiuza F, Hatarik R, Huntington CM, Kalantar DH, Link A et al (2017) Transition from collisional to collisionless regimes in interpenetrating plasma flows on the National Ignition Facility. Phys Rev Lett 118:185003. https://doi.org/10.1103/PhysRevLett.118.185003

Ruszkowski M, Yang HYK, Zweibel E (2017) Global simulations of galactic winds including cosmic-ray streaming. ApJ 834:208. https://doi.org/10.3847/1538-4357/834/2/208. arXiv:1602.04856

Ruyer C, Fiuza F (2018) Disruption of current filaments and isotropization of the magnetic field in counterstreaming plasmas. Phys Rev Lett 120:245002. https://doi.org/10.1103/PhysRevLett.120.245002. arXiv: 1805.09814

Ruyer C, Gremillet L, Bonnaud G (2015) Weibel-mediated collisionless shocks in laser-irradiated dense plasmas: prevailing role of the electrons in generating the field fluctuations. Phys Plasmas 22:082107. https://doi.org/10.1063/1.4928096

Ruyer C, Gremillet L, Bonnaud G, Riconda C (2016) Analytical predictions of field and plasma dynamics during nonlinear Weibel-mediated flow collisions. Phys Rev Lett 117:065001. https://doi.org/10.1103/ PhysRevLett.117.065001

Ryu D, Jones TW, Frank A (1995) Numerical magnetohydrodynamics in astrophysics: algorithm and tests for multidimensional flow. ApJ 452:785. https://doi.org/10.1086/176347. arXiv:astro-ph/9505073

Ryu D, Kim J, Hong SS, Jones TW (2003) The effect of cosmic-ray diffusion on the Parker instability. ApJ 589:338-346. https://doi.org/10.1086/374392. arXiv:astro-ph/0301625

Ryutov DD, Kugland NL, Park HS, Plechaty C, Remington BA, Ross JS (2012) Basic scalings for collisionless-shock experiments in a plasma without pre-imposed magnetic field. Plasma Phys Control Fusion 54:105021. https://doi.org/10.1088/0741-3335/54/10/105021

Sagdeev RZ (1966) Cooperative phenomena and shock waves in collisionless plasmas. Rev Plasma Phys $4: 23$

Sagdeev RZ, Shapiro VD (1973) Influence of transverse magnetic field on Landau damping. JETP Lett $17: 279$

Saito T, Hoshino M, Amano T (2013) Stability of cosmic-ray modified shocks: two-fluid approach. ApJ 775:130. https://doi.org/10.1088/0004-637X/775/2/130. arXiv:1307.7436

Salem M, Bryan GL (2014) Cosmic ray driven outflows in global galaxy disc models. MNRAS 437:33123330. https://doi.org/10.1093/mnras/stt2121. arXiv:1307.6215

Samtaney R, Loureiro NF, Uzdensky DA, Schekochihin AA, Cowley SC (2009) Formation of plasmoid chains in magnetic reconnection. Phys Rev Lett 103:105004. https://doi.org/10.1103/PhysRevLett. 103.105004. arXiv:0903.0542

Sanz-Serna JM, Calvo MP (1994) Numerical hamiltonian problems. Chapman \& Hall, London

Sarrat M, Ghizzo A, Del Sarto D, Serrat L (2017) Parallel implementation of a relativistic semi-Lagrangian Vlasov-Maxwell solver. Eur Phys J D. https://doi.org/10.1140/epjd/e2017-80188-4

Sarri G, Poder K, Cole JM, Schumaker W, Di Piazza A, Reville B, Dzelzainis T, Doria D, Gizzi LA, Grittani G, Kar S, Keitel CH, Krushelnick K, Kuschel S, Mangles SPD, Najmudin Z, Shukla N, Silva LO, Symes D, Thomas AGR, Vargas M, Vieira J, Zepf M (2015) Generation of neutral and high-density electron-positron pair plasmas in the laboratory. Nature Commun 6:6747 EP. https://doi.org/10.1038/ ncomms 7747

Schaeffer DB, Fox W, Haberberger D, Fiksel G, Bhattacharjee A, Barnak DH, Hu SX, Germaschewski K (2017a) Generation and evolution of high-mach-number laser-driven magnetized collisionless shocks in the laboratory. Phys Rev Lett 119:025001. https://doi.org/10.1103/PhysRevLett.119.025001 
Schaeffer DB, Fox W, Haberberger D, Fiksel G, Bhattacharjee A, Barnak DH, Hu SX, Germaschewski K, Follett RK (2017b) High-mach number, laser-driven magnetized collisionless shocks. Phys Plasmas 24:122702. https://doi.org/10.1063/1.4989562

Schlickeiser R (2002) Cosmic ray astrophysics. Astronomy and Astrophysics Library. Springer, Berlin. https://doi.org/10.1007/978-3-662-04814-6

Schlickeiser R, Dermer CD (2000) Proton and electron acceleration through magnetic turbulence in relativistic outflows. A\&A 360:789-794 arXiv:astro-ph/0005601

Schneider V, Katscher U, Rischke DH, Waldhauser B, Maruhn JA, Munz CD (1993) New algorithms for ultra-relativistic numerical hydrodynamics. J Comput Phys 105:92-107. https://doi.org/10.1006/jcph. 1993.1056

Scholer M, Matsukiyo S (2004) Nonstationarity of quasi-perpendicular shocks: a comparison of full particle simulations with different ion to electron mass ratio. Ann Geophys 22:2345-2353. https://doi.org/10. 5194/angeo-22-2345-2004

Schure KM, Achterberg A, Keppens R, Vink J (2010) Time-dependent particle acceleration in supernova remnants in different environments. MNRAS 406:2633-2649. https://doi.org/10.1111/j.1365-2966. 2010.16857.x. arXiv:1004.2766

Schure KM, Bell AR, O'C Drury L, Bykov AM (2012) Diffusive shock acceleration and magnetic field amplification. Space Sci Rev 173:491-519. https://doi.org/10.1007/s11214-012-9871-7. arXiv:1203.1637

Schwartz DA, Marshall HL, Lovell JEJ, Murphy DW, Bicknell GV, Birkinshaw M, Gelbord J, Georganopoulos M, Godfrey L, Jauncey DL, Perlman ES, Worrall DM (2006) Chandra observations of magnetic fields and relativistic beaming in four quasar jets. ApJ 640:592-602. https://doi.org/10.1086/500102. arXiv:astro-ph/0601632

Sgattoni A, Fedeli L, Sinigardi S, Marocchino A, Macchi A, Weinberg V, Karmakar A (2015) Optimising PICCANTE_-an open-source particle-in-cell code for advanced simulations on tier-0 systems. ArXiv e-prints arXiv: $1503.02464 \mathrm{v} 2$

Shalchi A (ed) (2009b) Nonlinear cosmic ray diffusion theories. Astrophysics and Space Science Library, vol 362. Springer, Berlin. https://doi.org/10.1007/978-3-642-00309-7

Shalchi A (2009a) Diffusive shock acceleration in supernova remnants: on the validity of the Bohm limit. Astropart Phys 31:237-242. https://doi.org/10.1016/j.astropartphys.2009.01.007

Shapiro VD, Üçer D (2003) Shock surfing acceleration. Planet Space Sci 51:665-680. https://doi.org/10. 1016/S0032-0633(03)00102-8

Sharma P, Colella P, Martin DF (2010) Numerical implementation of streaming down the gradient: application to fluid modeling of cosmic rays and saturated conduction. SIAM J Sci Comput 32:3564-3583. https://doi.org/10.1137/100792135

Shimada N, Hoshino M (2000) Strong electron acceleration at high mach number shock waves: simulation study of electron dynamics. ApJ 543:L67-L71. https://doi.org/10.1086/318161

Shoucri M, Matte JP, Vidal F (2015) Relativistic Eulerian Vlasov simulations of the amplification of seed pulses by Brillouin backscattering in plasmas. Phys Plasmas 22:053101. https://doi.org/10.1063/1. 4919614

Sironi L, Spitkovsky A (2009) Particle acceleration in relativistic magnetized collisionless pair shocks: dependence of shock acceleration on magnetic obliquity. ApJ 698:1523-1549. https://doi.org/10. 1088/0004-637X/698/2/1523. arXiv:0901.2578

Sironi L, Spitkovsky A (2011) Particle acceleration in relativistic magnetized collisionless electron-ion shocks. ApJ 726:75. https://doi.org/10.1088/0004-637X/726/2/75. arXiv:1009.0024

Sironi L, Spitkovsky A (2012) Particle-in-cell simulations of shock-driven reconnection in relativistic striped winds. Comput Sci Disc 5:014014. https://doi.org/10.1088/1749-4699/5/1/014014. arXiv:1208.4998

Sironi L, Spitkovsky A (2014) Relativistic reconnection: an efficient source of non-thermal particles. ApJ 783:L21. https://doi.org/10.1088/2041-8205/783/1/L21. arXiv:1401.5471

Sironi L, Spitkovsky A, Arons J (2013) The maximum energy of accelerated particles in relativistic collisionless shocks. ApJ 771:54. https://doi.org/10.1088/0004-637X/771/1/54. arXiv:1301.5333

Sironi L, Keshet U, Lemoine M (2015) Relativistic shocks: particle acceleration and magnetization. Space Sci Rev 191:519-544. https://doi.org/10.1007/s11214-015-0181-8. arXiv:1506.02034

Sironi L, Giannios D, Petropoulou M (2016) Plasmoids in relativistic reconnection, from birth to adulthood: first they grow, then they go. MNRAS 462:48-74. https://doi.org/10.1093/mnras/stw1620. arXiv: 1605.02071 
Sironi L, Beloborodov AM (2019) Kinetic simulations of radiative magnetic reconnection in the magnetized coronae of accreting black holes. arXiv e-prints arXiv:1908.08138

Skilling J (1975a) Cosmic ray streaming. I—effect of Alfvén waves on particles. MNRAS 172:557-566. https://doi.org/10.1093/mnras/172.3.557

Skilling J (1975b) Cosmic ray streaming. II-effect of particles on Alfvén waves. MNRAS 173:245-254. https://doi.org/10.1093/mnras/173.2.245

Smith AM, Gardiner CW (1989) Simulations of nonlinear quantum damping using the positive P representation. Phys Rev A 39:3511-3524. https://doi.org/10.1103/PhysRevA.39.3511

Snodin AP, Brandenburg A, Mee AJ, Shukurov A (2006) Simulating field-aligned diffusion of a cosmic ray gas. MNRAS 373:643-652. https://doi.org/10.1111/j.1365-2966.2006.11034.x. arXiv:astro-ph/0507176

Sol H, Pelletier G, Asseo E (1989) Two-flow model for extragalactic radio jets. MNRAS 237:411-429. https://doi.org/10.1093/mnras/237.2.411

Sonnendrücker E, Roche J, Bertrand P, Ghizzo A (1999) The semi-Lagrangian method for the numerical resolution of the Vlasov equation. J Comput Phys 149:201-220. https://doi.org/10.1006/jcph.1998. 6148

Spitkovsky A (2005) Simulations of relativistic collisionless shocks: shock structure and particle acceleration. In: Bulik T, Rudak B, Madejski G (eds) Astrophysical sources of high energy particles and radiation. AIP Conference Series, vol 801. American Institute of Physics, Melville, NY, pp 345-350. https://doi.org/10.1063/1.2141897. arXiv:astro-ph/0603211

Spitkovsky A (2008a) On the structure of relativistic collisionless shocks in electron-ion plasmas. ApJ 673:L39. https://doi.org/10.1086/527374. arXiv:0706.3126

Spitkovsky A (2008b) Particle acceleration in relativistic collisionless shocks: Fermi process at last? ApJ 682:L5. https://doi.org/10.1086/590248. arXiv:0802.3216

Stockem A, Fiuza F, Bret A, Fonseca RA, Silva LO (2014a) Exploring the nature of collisionless shocks under laboratory conditions. Sci Rep 4:3934. https://doi.org/10.1038/srep03934. arXiv:1401.2814

Stockem A, Grismayer T, Fonseca RA, Silva LO (2014b) Electromagnetic field generation in the downstream of electrostatic shocks due to electron trapping. Phys Rev Lett 113:105002. https://doi.org/10.1103/ PhysRevLett.113.105002. arXiv:1408.4608

Stone JM, Gardiner TA, Teuben P, Hawley JF, Simon JB (2008) Athena: a new code for astrophysical MHD. ApJS 178:137-177. https://doi.org/10.1086/588755. arXiv:0804.0402

Strauss RDT, Effenberger F (2017) A hitch-hiker's guide to stochastic differential equations. Space Sci Rev 212:151-192. https://doi.org/10.1007/s11214-017-0351-y. arXiv:1703.06192

Strokin NA (1985) Ion heating and energy redistribution in a collisionless shock wave. Zh Eksp Teor Fiz 88:2005-2014 [Sov. J. Plasma Phys. 10:313 (1985)]

Strong AW, Moskalenko IV (1998) Propagation of cosmic-ray nucleons in the galaxy. ApJ 509:212-228. https://doi.org/10.1086/306470. arXiv:astro-ph/9807150

Sturrock PA (1966) Stochastic acceleration. Phys Rev 141:186-191. https://doi.org/10.1103/PhysRev.141. 186

Sweet PA (1958) The neutral point theory of solar flares. In: Lehnert B (ed) Electromagnetic phenomena in cosmical physics. IAU symposium, vol 6. Cambridge University Press, p 123

Taflove A (2005) Computation electrodynamics: the finite-difference time-domain method, 3rd edn. Artech House, Norwood

Takabe H, Nagatomo H, Sunahara A, Ohnishi N, Mahdy AI, Yoda Y, Naruo S, Azechi H, Nishimura H, Mima K (1999) Recent studies of laser produced plasmas. Plasma Phys Control Fusion 41:A75-A97. https://doi.org/10.1088/0741-3335/41/3a/005

Takabe H, Kato TN, Sakawa Y, Kuramitsu Y, Morita T, Kadono T, Shigemori K, Otani K et al (2008) Highmach number collisionless shock and photo-ionized non-LTE plasma for laboratory astrophysics with intense lasers. Plasma Phys Control Fusion 50:124057. https://doi.org/10.1088/0741-3335/50/12/ 124057

Takahashi K, Yamada S, Yamada (2014) Exact Riemann solver for ideal magnetohydrodynamics that can handle all types of intermediate shocks and switch-on/off waves. J Plasma Phys 80:255-287. https:// doi.org/10.1017/S0022377813001268. arXiv:1310.2330

Telezhinsky I, Dwarkadas VV, Pohl M (2012a) Particle spectra from acceleration at forward and reverse shocks of young Type Ia Supernova Remnants. Astropart Phys 35:300-311. https://doi.org/10.1016/ j.astropartphys.2011.10.001. arXiv:1110.0361 
Telezhinsky I, Dwarkadas VV, Pohl M (2012b) Time-dependent escape of cosmic rays from supernova remnants, and their interaction with dense media. A\&A 541:A153. https://doi.org/10.1051/00046361/201118639. arXiv:1112.3194

Teyssier R (2002) Cosmological hydrodynamics with adaptive mesh refinement. A new high resolution code called RAMSES. A\&A 385:337-364. https://doi.org/10.1051/0004-6361:20011817. arXiv:astro-ph/0111367

Thomas T, Pfrommer C (2019) Cosmic-ray hydrodynamics: Alfvén-wave regulated transport of cosmic rays. MNRAS 485(3):2977-3008. https://doi.org/10.1093/mnras/stz263. arXiv:1805.11092

Thornbury A, Drury LO (2014) Power requirements for cosmic ray propagation models involving reacceleration and a comment on second-order Fermi acceleration theory. MNRAS 442:3010-3012. https://doi.org/10.1093/mnras/stu1080. arXiv:1404.2104

Toro EF (2009) Riemann solvers and numerical methods for fluid dynamics: a practical introduction, 3rd edn. Springer, Berlin. https://doi.org/10.1007/b79761

Toro EF, Spruce M, Speares W (1994) Restoration of the contact surface in the HLL-Riemann solver. Shock Waves 4:25-34. https://doi.org/10.1007/BF01414629

Torrilhon M (2003) Uniqueness conditions for Riemann problems of ideal magnetohydrodynamics. J Plasma Phys 69:253-276. https://doi.org/10.1017/S0022377803002186

Tóth G (2000) The $\nabla \cdot B=0$ constraint in shock-capturing magnetohydrodynamics codes. J Comput Phys 161:605-652. https://doi.org/10.1006/jcph.2000.6519

Tóth G, Sokolov IV, Gombosi TI, Chesney DR, Clauer CR, de Zeeuw DL, Hansen KC, Kane KJ, Manchester WB, Oehmke RC, Powell KG, Ridley AJ, Roussev II, Stout QF, Volberg O, Wolf RA, Sazykin S, Chan A, Yu B, Kóta J (2005) Space weather modeling framework: a new tool for the space science community. J Geophys Res 110(A9):A12226. https://doi.org/10.1029/2005JA011126

Tóth G, De Zeeuw DL, Gombosi TI, Powell KG (2006) A parallel explicit/implicit time stepping scheme on block-adaptive grids. J Comput Phys 217:722-758. https://doi.org/10.1016/j.jcp.2006.01.029

Tóth G, van der Holst B, Sokolov IV, De Zeeuw DL, Gombosi TI, Fang F, Manchester WB, Meng X, Najib D, Powell KG, Stout QF, Glocer A, Ma YJ, Opher M (2012) Adaptive numerical algorithms in space weather modeling. J Comput Phys 231:870-903. https://doi.org/10.1016/j.jcp.2011.02.006

Tóth G, Jia X, Markidis S, Peng IB, Chen Y, Daldorff LKS, Tenishev VM, Borovikov D, Haiducek JD, Gombosi TI, Glocer A, Dorelli JC (2016) Extended magnetohydrodynamics with embedded particlein-cell simulation of Ganymede's magnetosphere. J Geophys Res 121:1273-1293. https://doi.org/10. 1002/2015JA021997

Tregillis IL, Jones TW, Ryu D (2001) Simulating electron transport and synchrotron emission in radio galaxies: shock acceleration and synchrotron aging in three-dimensional flows. ApJ 557:475-491. https://doi.org/10.1086/321657. arXiv:astro-ph/0104305

Treumann RA, Jaroschek CH (2008a) Fundamentals of non-relativistic collisionless shock physics: II. Basic equations and models. ArXiv e-prints arXiv:0805.2162

Treumann RA, Jaroschek CH (2008b) Fundamentals of non-relativistic collisionless shock physics: V. Acceleration of charged particles. ArXiv e-prints arXiv:0806.4046

Uhlig M, Pfrommer C, Sharma M, Nath BB, Enßlin TA, Springel V (2012) Galactic winds driven by cosmic ray streaming. MNRAS 423:2374-2396. https://doi.org/10.1111/j.1365-2966.2012.21045.x. arXiv:1203.1038

Umeda T, Wada Y (2016) Secondary instabilities in the collisionless Rayleigh-Taylor instability: full kinetic simulation. Phys Plasmas 23:112117. https://doi.org/10.1063/1.4967859

Umeda T, Togano K, Ogino T (2009) Two-dimensional full-electromagnetic Vlasov code with conservative scheme and its application to magnetic reconnection. Comput Phys Commun 180:365-374. https:// doi.org/10.1016/j.cpc.2008.11.001

Umeda T, Ueno S, Nakamura TKM (2014) Ion kinetic effects on nonlinear processes of the KelvinHelmholtz instability. Plasma Phys Control Fusion 56:075006. https://doi.org/10.1088/0741-3335/ 56/7/075006

Umeda T, Yamazaki R, Ohira Y, Ishizaka N, Kakuchi S, Kuramitsu Y, Matsukiyo S, Miyata I et al (2019) Full particle-in-cell simulation of the interaction between two plasmas for laboratory experiments on the generation of magnetized collisionless shocks with high-power lasers. Phys Plasmas 26:032303. https://doi.org/10.1063/1.5079906

Usami S, Horiuchi R, Ohtani H, Den M (2014) Multi-hierarchy simulation of collisionless driven reconnection by real-space decomposition. J Phys: Conf Ser 561:012021. https://doi.org/10.1088/1742-6596/ $561 / 1 / 012021$ 
Usami S, Horiuchi R, Ohtani H, Ono Y, Tanabe H (2018) Effective proton heating through collisionless driven reconnection in the presence of guide field. Plasma Fusion Res 13:3401025-3401025. https:// doi.org/10.1585/pfr.13.3401025

Uzdensky DA (2016) Radiative magnetic reconnection in astrophysics. In: Gonzalez W, Parker E (eds) Magnetic reconnection: concepts and applications. Astrophysics and Space Science Library, vol 427. Springer, Cham, p 473. https://doi.org/10.1007/978-3-319-26432-5_12. arXiv:1510.05397

Uzdensky et al (2019) Extreme plasma astrophysics. arXiv:1903.05328

Uzdensky DA, Spitkovsky A (2014) Physical conditions in the reconnection layer in pulsar magnetospheres. ApJ 780:3. https://doi.org/10.1088/0004-637X/780/1/3. arXiv:1210.3346

Uzdensky DA, Cerutti B, Begelman MC (2011) Reconnection-powered linear accelerator and gammaray flares in the Crab Nebula. ApJ 737:L40. https://doi.org/10.1088/2041-8205/737/2/L40. arXiv:1105.0942

Vaidya B, Mignone A, Bodo G, Rossi P, Massaglia S (2018) A particle module for the PLUTO Code. II. Hybrid framework for modeling nonthermal emission from relativistic magnetized flows. ApJ 865:144. https://doi.org/10.3847/1538-4357/aadd17. arXiv:1808.08960

Valentini F, Trávníček P, Califano F, Hellinger P, Mangeney A (2007) A hybrid-Vlasov model based on the current advance method for the simulation of collisionless magnetized plasma. J Comput Phys 225:753-770. https://doi.org/10.1016/j.jcp.2007.01.001

van der Holst B, Keppens R (2007) Hybrid block-AMR in cartesian and curvilinear coordinates: MHD applications. J Comput Phys 226:925-946. https://doi.org/10.1016/j.jcp.2007.05.007

van Leer B (1979) Towards the ultimate conservative difference scheme. V. A second-order sequel to Godunov's method. J Comput Phys 32:101-136. https://doi.org/10.1016/0021-9991(79)90145-1

van Marle AJ, Casse F, Marcowith A (2018) On magnetic field amplification and particle acceleration near non-relativistic astrophysical shocks: particles in MHD cells simulations. MNRAS 473:3394-3409. https://doi.org/10.1093/mnras/stx2509. arXiv:1709.08482

van Marle AJ, Casse F, Marcowith A (2019) Three-dimensional simulations of non-resonant streaming instability and particle acceleration near non-relativistic astrophysical shocks. MNRAS 490(1):11561165. https://doi.org/10.1093/mnras/stz2624. arXiv:1909.06931

Vanthieghem A, Lemoine M, Gremillet L (2018) Stability analysis of a periodic system of relativistic current filaments. Phys Plasmas 25:072115. https://doi.org/10.1063/1.5033562. arXiv:1804.04429

Vanthieghem A, Lemoine M, Plotnikov I, Grassi A, Grech M, Gremillet L, Pelletier G (2020) Physics and phenomenology of weakly magnetized, relativistic astrophysical shock waves. ArXiv e-prints arXiv:2002.01141

Vay JL (2008) Simulation of beams or plasmas crossing at relativistic velocity. Phys Plasmas 15:056701. https://doi.org/10.1063/1.2837054

Vay JL, Geddes CGR, Cormier-Michel E, Grote DP (2011) Numerical methods for instability mitigation in the modeling of laser wakefield accelerators in a Lorentz-boosted frame. J Comput Phys 230:59085929. https://doi.org/10.1016/j.jcp.2011.04.003

Vay JL, Haber I, Godfrey BB (2013) A domain decomposition method for pseudo-spectral electromagnetic simulations of plasmas. J Comput Phys 243:260-268. https://doi.org/10.1016/j.jcp.2013.03.010

Verboncoeur JP (2005) Particle simulation of plasmas: review and advances. Plasma Phys Control Fusion 47(5A):A231

Vietri M (1995) The acceleration of ultra-high-energy cosmic rays in gamma-ray bursts. ApJ 453:883. https://doi.org/10.1086/176448. arXiv:astro-ph/9506081

Vincenti H, Vay JL (2018) Ultrahigh-order maxwell solver with extreme scalability for electromagnetic pic simulations of plasmas. Comput Phys Commun 228:22-29. https://doi.org/10.1016/j.cpc.2018. 03.018

Vink J (2012) Supernova remnants: the X-ray perspective. A\&A Rev 20:49. https://doi.org/10.1007/s00159011-0049-1. arXiv:1112.0576

Vladimirov A, Ellison DC, Bykov A (2006) Nonlinear diffusive shock acceleration with magnetic field amplification. ApJ 652:1246-1258. https://doi.org/10.1086/508154. arXiv:astro-ph/0606433

Vurm I, Poutanen J (2009) Time-dependent modeling of radiative processes in hot magnetized plasmas. ApJ 698:293-316. https://doi.org/10.1088/0004-637X/698/1/293. arXiv:0807.2540

Vurm I, Beloborodov AM, Poutanen J (2011) Gamma-ray bursts from magnetized collisionally heated jets. ApJ 738:77. https://doi.org/10.1088/0004-637X/738/1/77. arXiv:1104.0394 
Wagner AY, Falle SAEG, Hartquist TW (2007) Two-fluid models of cosmic-ray modified radiative shocks including the effects of an acoustic instability. A\&A 463:195-201. https://doi.org/10.1051/0004-6361: 20066307

Wagner AY, Lee JJ, Raymond JC, Hartquist TW, Falle SAEG (2009) A cosmic-ray precursor model for a balmer-dominated shock in Tycho's supernova remnant. ApJ 690:1412-1423. https://doi.org/10. 1088/0004-637X/690/2/1412. arXiv:0809.2504

Wallin E, Gonoskov A, Marklund M (2015) Effects of high energy photon emissions in laser generated ultra-relativistic plasmas: real-time synchrotron simulations. Phys Plasmas 22:033117. https://doi.org/ 10.1063/1.4916491. arXiv:1409.5574

Wang C, Lin T, Caflisch R, Cohen BI, Dimits AM (2008) Particle simulation of Coulomb collisions: comparing the methods of Takizuka \& Abe and Nanbu. J Comput Phys 227:4308-4329. https://doi. org/10.1016/j.jcp.2007.12.027

Wang L, Hakim AH, Bhattacharjee A, Germaschewski K (2015) Comparison of multi-fluid moment models with particle-in-cell simulations of collisionless magnetic reconnection. Phys Plasmas 22:012108. https://doi.org/10.1063/1.4906063. arXiv:1409.0262

Warren DC, Ellison DC, Bykov AM, Lee SH (2015) Electron and ion acceleration in relativistic shocks with applications to GRB afterglows. MNRAS 452:431-443. https://doi.org/10.1093/mnras/stv1304. arXiv: 1506.03087

Warwick J, Dzelzainis T, Dieckmann ME, Schumaker W, Doria D, Romagnani L, Poder K, Cole JM et al (2017) Experimental observation of a current-driven instability in a neutral electron-positron beam. Phys Rev Lett 119:185002. https://doi.org/10.1103/PhysRevLett.119.185002

Waxman E (1995) Cosmological gamma-ray bursts and the highest energy cosmic rays. Phys Rev Lett 75:386-389. https://doi.org/10.1103/PhysRevLett.75.386. arXiv:astro-ph/9505082

Waxman E (1997) Gamma-ray-burst afterglow: supporting the cosmological fireball model, constraining parameters, and making predictions. ApJ 485:L5-L8. https://doi.org/10.1086/310809. arXiv:astro-ph/9704116

Webb GM, Axford WI, Terasawa T (1983) On the drift mechanism for energetic charged particles at shocks. ApJ 270:537-553. https://doi.org/10.1086/161146

Webb GM, Drury LO, Biermann P (1984) Diffusive shock acceleration of energetic electrons subject to synchrotron losses. A\&A 137:185-201

Webb GM, Drury LO, Volk HJ (1986) Cosmic-ray shock acceleration in oblique MHD shocks. A\&A 160:335-346

Weibel ES (1959) Spontaneously growing transverse waves in a plasma due to an anisotropic velocity distribution. Phys Rev Lett 2:83-84. https://doi.org/10.1103/PhysRevLett.2.83

Weidl MS, Heuer P, Schaeffer D, Dorst R, Winske D, Constantin C, Niemann C (2017) Towards a parallel collisionless shock in LAPD. J Phys: Conf Ser 900:012020. https://doi.org/10.1088/1742-6596/900/ 1/012020

Wentzel DG (1963) Fermi acceleration of charged particles. ApJ 137:135. https://doi.org/10.1086/147490

Werner GR, Uzdensky DA, Begelman MC, Cerutti B, Nalewajko K (2018) Non-thermal particle acceleration in collisionless relativistic electron-proton reconnection. MNRAS 473:4840-4861. https://doi.org/10. 1093/mnras/stx2530. arXiv:1612.04493

Werner GR, Philippov AA, Uzdensky DA (2019) Particle acceleration in relativistic magnetic reconnection with strong inverse-Compton cooling in pair plasmas. MNRAS 482:L60-L64. https://doi.org/10.1093/ mnrasl/sly157. arXiv:1805.01910

West JL, Jaffe T, Ferrand G, Safi-Harb S, Gaensler BM (2017) When disorder looks like order: a new model to explain radial magnetic fields in young supernova remnants. ApJ 849:L22. https://doi.org/10.3847/ 2041-8213/aa94c4. arXiv:1711.02176

Whipple EC, Northrop TG, Birmingham TJ (1986) Adiabatic theory in regions of strong field gradients. J Geophys Res 91:4149-4156. https://doi.org/10.1029/JA091iA04p04149

Wieland V, Pohl M, Niemiec J, Rafighi I, Nishikawa KI (2016) Nonrelativistic perpendicular shocks modeling young supernova remnants: nonstationary dynamics and particle acceleration at forward and reverse shocks. ApJ 820:62. https://doi.org/10.3847/0004-637X/820/1/62. arXiv:1602.05064

Willingale L, Nilson PM, Kaluza MC, Dangor AE, Evans RG, Fernandes P, Haines MG, Kamperidis C, Kingham RJ, Ridgers CP, Sherlock M, Thomas AGR, Wei MS, Najmudin Z, Krushelnick K, Bandyopadhyay S, Notley M, Minardi S, Tatarakis M, Rozmus W (2010) Proton deflectometry of a magnetic reconnection geometry. Phys Plasmas 17:043104. https://doi.org/10.1063/1.3377787 
Wilson F, Neukirch T, Allanson O (2017) Force-free collisionless current sheet models with non-uniform temperature and density profiles. Phys Plasmas 24:092105. https://doi.org/10.1063/1.4997703

Winske D, Gary SP (2007) Hybrid simulations of debris-ambient ion interactions in astrophysical explosions. J Geophys Res. https://doi.org/10.1029/2007JA012276

Wong K, Zhdankin V, Uzdensky DA, Werner GR, Begelman MC (2019) First-principles demonstration of diffusive particle acceleration in kinetic simulations of relativistic plasma turbulence. ArXiv e-prints arXiv: 1901.03439

Wright TP (1972) Comments on "demonstration of collisionless interactions between interstreaming ions in a laser-produced-plasma experiment”. Phys Rev Lett 28:268-270. https://doi.org/10.1103/ PhysRevLett.28.268

Wu CS, Winske D, Zhou YM, Tsai ST, Rodriguez P, Tanaka M, Papadopoulos K, Akimoto K, Lin CS, Leroy MM, Goodrich CC (1984) Microinstabilities associated with a high Mach number, perpendicular bow shock. Space Sci Rev 37:63-109. https://doi.org/10.1007/BF00213958

Yamada M, Ji H, Hsu S, Carter T, Kulsrud R, Bretz N, Jobes F, Ono Y, Perkins F (1997) Study of driven magnetic reconnection in a laboratory plasma. Phys Plasmas 4:1936-1944. https://doi.org/10.1063/1. 872336

Yamazaki R, Yoshida T, Tsuchihashi Y, Nakajima R, Ohira Y, Yanagita S (2015) Electron acceleration with improved stochastic differential equation method: cutoff shape of electron distribution in test-particle limit. J High Energy Astrophys 5:1-8. https://doi.org/10.1016/j.jheap.2015.02.001. arXiv:1502.05918

Zank GP, Pauls HL, Cairns IH, Webb GM (1996) Interstellar pickup ions and quasi-perpendicular shocks: implications for the termination shock and interplanetary shocks. J Geophys Res 101:457-478. https:// doi.org/10.1029/95JA02860

Zanotti O, Dumbser M (2011) Numerical simulations of high Lundquist number relativistic magnetic reconnection. MNRAS 418:1004-1011. https://doi.org/10.1111/j.1365-2966.2011.19551.x. arXiv:1103.5924

Zenitani S (2015) Loading relativistic Maxwell distributions in particle simulations. Phys Plasmas 22:042116. https://doi.org/10.1063/1.4919383

Zenitani S, Hoshino M (2001) The generation of nonthermal particles in the relativistic magnetic reconnection of pair plasmas. ApJ 562:L63-L66. https://doi.org/10.1086/337972. arXiv:1402.7139

Zenitani S, Hoshino M (2005a) Relativistic particle acceleration in a folded current sheet. ApJ 618:L111L114. https://doi.org/10.1086/427873. arXiv:astro-ph/0411373

Zenitani S, Hoshino M (2005b) Three-dimensional evolution of a relativistic current sheet: triggering of magnetic reconnection by the guide field. Phys Rev Lett 95:095001. https://doi.org/10.1103/PhysRevLett. 95.095001. arXiv:astro-ph/0505493

Zenitani S, Hoshino M (2007) Particle acceleration and magnetic dissipation in relativistic current sheet of pair plasmas. ApJ 670:702-726. https://doi.org/10.1086/522226. arXiv:0708.1000

Zenitani S, Hoshino M (2008) The role of the guide field in relativistic pair plasma reconnection. ApJ 677:530-544. https://doi.org/10.1086/528708. arXiv:0712.2016

Zhang M (2000) Calculation of diffusive shock acceleration of charged particles by skew Brownian motion. ApJ 541:428-435. https://doi.org/10.1086/309429

Zhang M, Lee MA (2013) Stochastic acceleration of energetic particles in the heliosphere. Space Sci Rev 176:133-146. https://doi.org/10.1007/s11214-011-9754-3

Zharkova VV, Arzner K, Benz AO, Browning P, Dauphin C, Emslie AG, Fletcher L, Kontar EP, Mann G, Onofri M, Petrosian V, Turkmani R, Vilmer N, Vlahos L (2011) Recent advances in understanding particle acceleration processes in solar flares. Space Sci Rev 159:357-420. https://doi.org/10.1007/ s11214-011-9803-y. arXiv: 1110.2359

Zhdankin V, Werner GR, Uzdensky DA, Begelman MC (2017) Kinetic turbulence in relativistic plasma: from thermal bath to nonthermal continuum. Phys Rev Lett 118:055103. https://doi.org/10.1103/ PhysRevLett.118.055103. arXiv:1609.04851

Zhdankin V, Uzdensky DA, Werner GR, Begelman MC (2018) System-size convergence of nonthermal particle acceleration in relativistic plasma turbulence. ApJ 867:L18. https://doi.org/10.3847/20418213/aae88c. arXiv: 1805.08754

Zhdankin V, Uzdensky DA, Werner GR, Begelman MC (2019) Electron and ion energization in relativistic plasma turbulence. Phys Rev Lett 122(5):055101. https://doi.org/10.1103/PhysRevLett.122.055101. arXiv: 1809.01966 
Zhong J, Li Y, Wang X, Wang J, Dong Q, Xiao C, Wang S, Liu X, Zhang L, An L, Wang F, Zhu J, Gu Y, He X, Zhao G, Zhang J (2010) Modelling loop-top X-ray source and reconnection outflows in solar flares with intense lasers. Nature Phys 6:984 EP. https://doi.org/10.1038/nphys1790

Zirakashvili VN, Ptuskin VS (2008) Diffusive shock acceleration with magnetic amplification by nonresonant streaming instability in supernova remnants. ApJ 678:939-949. https://doi.org/10.1086/529580. arXiv:0801.4488

Zirakashvili VN, Ptuskin VS (2012) Numerical simulations of diffusive shock acceleration in SNRs. Astropart Phys 39:12-21. https://doi.org/10.1016/j.astropartphys.2011.09.003. arXiv:1109.4482

Zirakashvili VN, Breitschwerdt D, Ptuskin VS, Voelk HJ (1996) Magnetohydrodynamic wind driven by cosmic rays in a rotating galaxy. A\&A 311:113-126

Zweibel EG, Yamada M (2009) Magnetic reconnection in astrophysical and laboratory plasmas. ARA\&A 47:291-332. https://doi.org/10.1146/annurev-astro-082708-101726

Publisher's Note Springer Nature remains neutral with regard to jurisdictional claims in published maps and institutional affiliations.

\section{Affiliations}

\section{Alexandre Marcowith ${ }^{1}$ - Gilles Ferrand ${ }^{2} \cdot$ Mickael Grech $^{3} \cdot$ Zakaria Meliani $^{4}$. Illya Plotnikov ${ }^{5}$. Rolf Walder 6}

$凶 \quad$ Alexandre Marcowith

Alexandre.Marcowith@umontpellier.fr

1 Laboratoire Univers et Particules de Montpellier (LUPM), CNRS/IN2P3, CC72, Université Montpellier, Place Eugène Bataillon, 34095 Montpellier Cedex 5, France

2 Astrophysical Big Bang Laboratory (ABBL), RIKEN Cluster for Pioneering Research, and Interdisciplinary Theoretical and Mathematical Sciences Program (iTHEMS), RIKEN, Wakō, Saitama 351-0198, Japan

3 Laboratoire d'Utilisation des Lasers Intenses (LULI), CNRS, Ecole Polytechnique, Sorbonne Université, CEA, 91128 Palaiseau cedex, France

4 LUTH, Observatoire de Paris, PSL Research University, CNRS UMR 8102, Université Paris Diderot, Meudon, France

5 IRAP, Université de Toulouse, UPS-OMP, 9 av. Colonel Roche, BP 44346, 31028 Toulouse Cedex 4, France

6 École Normale Supérieur de Lyon, CNRS, Centre de Recherche d'Astrophysique de Lyon (CRAL), UMR 5574, Université de Lyon, Lyon, France 